\title{
Leach Testing of Simulated Hanford Waste Vitrification Plant Reference Glass HW-39
}

\author{
S. O. Bates \\ G. F. Piepel \\ J. W. Johnston
}

May 1989

Prepared for the U.S. Department of Energy under Contract DE-AC06-76RLO 1830

Pacific Northwest Laboratory

Operated for the U.S. Department of Energy by Battelle Memorial Institute 


\title{
DISCLAIMER
}

This report was prepared as an account of work sponsored by an agency of the United States Government. Neither the United States Government nor any agency thereof, nor Battelle Memorial Institute, nor any or their employees, makes any warranty, expressed or implied, or assumes any legal liability or responsibility for the accuracy, completeness, or usefulness of any information, apparatus, product, or process disclosed, or represents that its use would not infringe privately owned rights. Reference herein to any specific commercial product, process, or service by trade name, trademark, manufacturer, or otherwise does not necessarily constitute or imply its endorsement, recommendation, or favoring by the United States Government or any agency thereof, or Battelle Memorial Institute. The views and opinions of authors expressed herein do not necessarily state or reflect those of the United States Government or any agency thereof.

\author{
PACIFIC NORTHWEST LABORATORY \\ operated by \\ BATTELLE MEMORIAL INSTITUTE \\ for the \\ UNITED STATES DEPARTMENT OF ENERGY \\ under Contract DE-AC06-76RLO 1830
}

Printed in the United States of America
Available from
National Technical Intormation Service
United States Department of Commerce
5285 Port Royal Road
Springtield, Virginia 22161
NTIS Price Codes
Microriche A01
Printed Copy
Pages
$001-025$
$026-050$




\author{
S. 0. Bates \\ G. F. Piepel \\ J. W. Johnston
}

May 1989

Prepared for

the U.S. Department of Energy

under Contract DE-ACO6-76RLO 1830

Pacific Northwest Laboratory

Richland, Washington 99352 


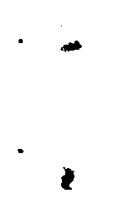




\section{SUMMARY}

This report summarizes the work performed to investigate the viability of a leach testing methodology for the Hanford Waste Vitrification Plant (HWVP) and provide glass dissolution data for HWVP model determination and validation. Leach tests up to one year in duration were conducted on the HWVP reference glass HW-39-1 (1.3 wt\% $\left.\mathrm{Cr}_{2} \mathrm{O}_{3}\right)$, using the Materials Characterization Center Static Leach Test (MCC-1) and the Agitated Powder Leach Test (MCC-3). Glass surface area to leachate volume (SA/V) ratios of $10,530,2,000$, and $20,000 \mathrm{~m}^{-1}$ were investigated during testing. The data resulting from the leach tests were statistically analyzed to evaluate the testing methodology. Based on these analyses, changes are recommended in the leach testing methodology, including changes in the randomization and replication $p l a n s$ to provide for better statistical characterization of glass durability.

The statistical evaluation of the test data found that the current leach testing methodology provides the necessary data for calculating some of the sources of variability in the test results, but not others. Proposed additions and modifications to the testing methodology will provide for collecting the data required to estimate the other variance components so that defensible statistical qualification statements can be made.

Results from a modified MCC-3 leach testing technique were compared with the results generated from the MCC-1 and MCC-3 techniques used in this study. The modified MCC-3 test is a partial leachant replacement MCC-3 leach test in which one leach vesse 1 is used for each $S A / V$ condition tested. Leachate samples are removed at different time intervals and the same amount of fresh leachant is added to the vessel. The modified MCC-3 test technique generated test conditions and data very similar to that generated by the standard MCC -1 and MCC -3 techniques. Because the modified MCC-3 technique provides data faster, requires less sample preparation, and costs less to perform, it is recommended that the modified MCC -3 techniques be used as a major part of future leach testing of HWVP glass. 


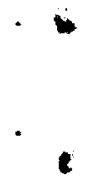




\section{CONTENTS}

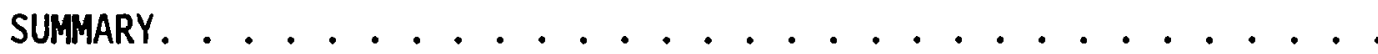

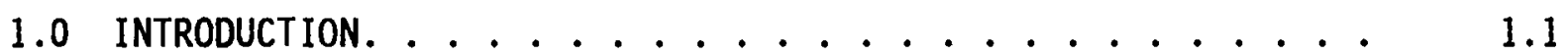

1.1 OBJECTIVES . . . . . . . . . . . . . . 1.2

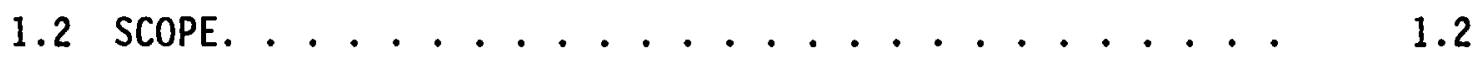

2.0 TECHNICAL APPROACH. .......................... 2.1

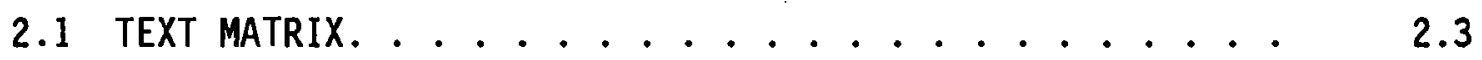

2.2 QUALITY ASSURANCE. . . . . . . . . . . . . 2.6

3.0 RESULTS . . . . . . . . . . . . . . . . 3.1

4.0 DISCUSSION. .......................... 4.1

4.1 SA/V SCALING ............... 4.1

4.2 CONGRUENT DISSOLUTION. . . . . . . . . . 4.3

4.3 FORWARD AND STEADY-STATE DISSOLUTION RATES . . . . . . 4.5

4.4 STATISTICAL EVALUATION . . . . . . . . . . . . 4.10

4.5 RECOMMENDATIONS FOR CHANGES TO THE LEACH TESTING

4.5.1 Parallel Testing with an "Appropriate
Certified Standard Glass" . . . . . . . 4.15

4.5.2 Replication and Randomization........ 4.16

4.5.3 Glass Analysis--Short- and Long-Term
Within-Laboratory Variation ....... 4.17

4.5.4 Glass Analysis--Lab-to-Lab Variation. . . . . 4.18

4.5.5 Leach Testing and Leachate Analysis--Short-
and Long-Term Within-Laboratory Variation.... 4.18

4.5.6 Leach Testing and Leachate Analysis--Lab-
to-Lab Variation. . . . . . . . 4.20

5.0 COMPARISON WITH MODIFIED MCC-3 TESTING. . . . . . . . . 5.1

6.0 CONCLUSIONS . . . . . . . . . . . . . . . 6.1 
7.0 RECOMMENDATIONS . . . . . . . . . . . . . . . . .

7.1

8.0 REFERENCES. . . . . . . . . . . . . . . . . 8.1

APPENDIX A - TABLES OF ELEMENTAL AND NORMALIZED

CONCENTRATION RESULTS ................ A.1

APPENDIX B - QUANTIFYING THE UNCERTAINTY IN

NORMALIZED CONCENTRATIONS ............. B. 1 


\section{FIGURES}

2.1 Resultant $\log [(S A / V) \cdot t]$ Values for Each Type of Test in the Test Matrix

3.1 Plot of Normalized Concentrations Versus (SA/V) -t from

MCC - 1 Leach Tests of HW-39-1 at an SA/V Ratio of $10 \mathrm{~m}^{-1}$. .

3.2 Plot of Normalized Concentrations Versus (SA/V) - t from

MCC -3 Leach Tests of HW-39-1 at an SA/V Ratio of $530 \mathrm{~m}^{-1}$. .

3.3 Plot of Normalized Concentrations Versus (SA/V) - t from MCC -3 Leach Tests of HW-39-1 at an SA/V Ratio of 2,000 $\mathrm{m}^{-1}$. .

3.4 Plot of Normalized Concentrations Versus (SA/V) $\cdot t$ from MCC -3 Leach Tests of HW-3-1 at an SA/V Ratio of $20,000 \mathrm{~m}^{-1}$. .

3.5 Plot of Normalized Boron Concentrations Versus (SA/V) - $t$ from HW-39-1 Leach Tests. . . . . . . . . . .

3.6 Plot of Normalized Silicon Concentration Versus $(S A / V) \cdot t$ from HW-39-1 Leach Tests. . . . . . . . . . . .

4.1 Plot of Calculated $90^{\circ} \mathrm{C}$ pH Values Versus (SA/V) $\cdot t$ from HW-39-1 Leach Tests ................

4.2 Normalized Concentratjon Versus (SA/V) - t from HW-39 Leach Tests at $S A / V=10 \mathrm{~m}^{-1}, 90^{\circ} \mathrm{C}$, in Deionized Water at 0.25 ,

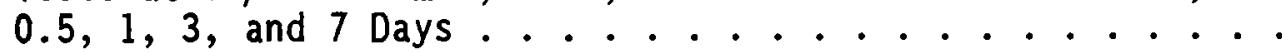

4.3 Ratios of the Normalized Concentration of Each Element to the Normalized Concentration of Silicon Versus (SA/V) $\cdot t$ for $S A / V$ Ratios of $10 \mathrm{~m}^{-1}$. . . . . . . . . . .

4.4 Typical Release Curve for Highly Soluble Glass Components, Such as Boron .............

4.5 Log-Log Plot of Typical Release Data for Highly Soluble Glass Components Such As Boron. ..........

4.6 Normalized Boron Concentration Versus (SA/V) - t for HW-39-1 with Forward and Steady-State Dissolution Rates...

5.1 Comparison of Normalized Boron Concentrations from HW-39-1 Product Leach Test Strategy and a Modified MCC -3 Methodology . . . . . . . . . . .

5.2 Comparison of Normalized Silicon Concentrations from HW-39-1 Product Leach Test Strategy and a Modified MCC - 3 Methodology 


\section{TABLES}

2.1 Composition of the HWVP Reference Waste, Substituted Waste, and Glass at a 25 wt\% 0xide Waste Loading . . . . . . . 2.2

2.2 Leaching Test Matrix for HWVP Waste Form Qualification. . . . 2.4

2.3 Glass and Leachate Amounts used to Produce Required SA/V Ratios in MCC-3 Testing ............. 2.6

4.1 Illustration of the Nine Variance Components that must be Estimated to make Defensible Qualification Statements . . . 4 4.11

4.2 Comparison of Tolerance and Confidence Intervals for the Al Example Considered in Appendix B .......... 4.14 


\subsection{INTRODUCTION}

The Hanford Waste Vitrification Plant (HWVP) will convert Hanford defense liquid high-level and transuranic (TRU) wastes to a solid vitrified (borosilicate glass) waste form suitable for final disposal in a geologic repository. The Department of Energy's Office of Civilian Radioactive Waste Management (DOE-OCRWM) is in the process of developing Waste Acceptance Specifications (WAS) for the high-level radioactive waste forms to be produced at the Defense Waste Processing Facility (DWPF)(1) and the West Valley Demonstration Project (WVDP).(2) The specifications that define the suitability of the HWVP waste form are expected to be very similar. In accordance with the waste acceptance process established by OCRWM in 1985, durability (leach) testing to determine elemental release and dissolution characteristics will be performed by both the candidate repository and the high-level waste producers. The waste producer will conduct leach testing to show compliance with the WAS; the purpose of repository testing will be to show compliance with the repository post-closure performance specified in 1OCFR60, i.e., releases of less than 1 part in $10^{5}$ per year. (3)

The high-level waste producers are proceeding with plant design and construction while simultaneously developing approaches for verifying the radionuclide release characteristics of their waste forms. Accordingly, the HWVP intends to 1) qualify its product through the testing of prototypic glasses produced during laboratory- and pilot-scale tests and cold startup operation of the HWVP and 2) ensure that production glass is within the range of qualified behavior.

Where possible, compliance with the WAS will be achieved by direct measurement of the applicable process or product variables and administrative control of the HWVP. Where direct measurements are not possible or are limited (e.g., glass durability/dissolution characteristics), translation of available direct measurements (e.g., feed composition) for comparison to the required WA will be achieved through process and product models. HWVP will define a region of acceptable glass compositions based on cold and limited radioactive laboratory testing (i.e., a product mode1). A process model will be developed to predict product glass composition as a function of measurable 
process control parameters. Based on operation within specified process control parameters, the HWVP will produce acceptable glass.

Data from the HWVP leach testing reported herein and other testing will be used in developing product and process models. These models and the actual plant operating data will then be the bases for demonstrating product compliance with the WAS requirements during cold and hot verification.

A leach testing methodology was developed to provide a base of glass dissolution properties to support process and product model development as part of waste form qualification (WFQ) activities. (4) This methodology uses leach tests conducted at increased glass surface area to leachate volume $(S A / V)$ ratios and shorter duration to approximate test results from lower $S A / V$ ratios and longer duration (equivalent $[S A / V] \cdot t$ values). The tests were conducted under $Q A$ requirements commensurate with those of other licensingrelated programs conducted at Pacific Northwest Laboratory (PNL)(a). These QA requirements will also apply during actual waste form qualification leach testing.

\subsection{OBJECTIVES}

There were two major objectives for this set of leach tests. The first was to generate data that describe the behavior of the HWVP reference glass and that can be used to develop a rate law for the dissolution of the glass. The other objective was to evaluate the applicability of this leach testing for use in the HWVP waste form qualification and glass dissolution modeling. Changes in the methodology that would increase the efficiency, characterization, and defensibility of the testing procedure and the resulting data base are also presented as recommendations. The data generated from these tests were also compared with another testing methodology using a modified MCC-3 technique.

(a) Pacific Northwest Laboratory is operated for the U.S. Department of Energy by Battelle Memorial Institute under contract DE-AC06-76RLO 1830. 


\subsection{SCOPE}

The testing methodology used in this study was proposed in order to characterize the leaching performance, i.e., chemical durability, of the HWVP nominal glass composition to be produced in the HWVP. The leachate used for these leaching studies was deionized water. Ground water or materials typical of a repository were not used. Thus, this testing methodology provides a generic data base that can be combined with repository program test data to evaluate the HWVP waste form. In a repository, the release of many of the glass components, including the major radionuclides, is controlled by solubilities and mass transfer behavior in the vicinity of the waste package. A repository environment with very small ground water flow rates is more benign than conditions in the laboratory tests in deionized (silica free) water. (5) Actual ground waters have high silica concentrations, whereas silica accumulation in deionized water occurs only after long times in static leach tests. The rate of glass dissolution in deionized water will be greatest initially because the dissolution process is not inhibited by high concentrations of silica, as is the case in many ground waters. 
. 


\subsection{IECHNICAL APPROACH}

One of the main purposes of this leach testing study was to evaluate the applicability of a leach testing strategy that uses tests conducted at increased $S A / V$ ratios and shorter durations to approximate test results from lower $S A / V$ ratios and longer duration (equivalent $[S A / V] \cdot t$ values) with a deionized water (DIW) leachate. The required testing necessary to evaluate the methodology was conducted using a nonradioactive HWVP reference glass. Glass of the HWVP reference composition HW-39-1 (1.3 wt\% $\mathrm{Cr}_{2} \mathrm{O}_{3}$ ), which incorporated simulated Neutralized Current Acid Waste (NCAW'84), was produced in the laboratory and used for all leach tests. The compositions of the NCAW'84 (high- $\mathrm{Cr}_{2} \mathrm{O}_{3}$ ) waste and the $\mathrm{HW}-39-1 \mathrm{glass}$ are shown in Table 2.1. Testing was conducted using the MCC Static Leach Test (MCC-1) ${ }^{(6)}$ and the MCC Agitated Powder Leach Test (MCC-3) (7) methods.

The MCC -1 leach test measures the elemental mass loss from a monolithic sample of glass as a function of time. A glass sample is suspended on a Teflon(a) support and sealed in a Teflon container. The SA/V ratio used was $10 \mathrm{~m}^{-1}$. Tests reported herein were conducted at $90^{\circ} \mathrm{C}$ using deionized water as the leachant.

The MCC -3 test uses powdered glass instead of the monolithic sample used in the MCC-1 test. The MCC-3 tests are intended to yield results that would be similar to results from monolith tests conducted for extended periods of time. These tests can be used to represent advanced stages of waste form dissolution where leachant saturation is at or above reaction product solubility limits and the composition of the dissolved glass matrix dominates the chemistry of the deionized water leachant. The conditions where solubility effects predominate occur more rapidly in MCC -3 tests because the increase in the surface area exposed to leachant results in increased amounts of glass components in solution.

(a) Teflon is a registered trademark of the E. I. Du Pont de Nemours and Company. 
IABLE 2.1. Composition of the HWVP Reference Waste (NCAW'84, 5.3 wt\% $\mathrm{Cr}_{2} \mathrm{O}_{3}$ ), Substituted Waste, and Glass (HW-39-1) at a $25 \mathrm{wt} \%$ Oxide Waste Loading

\begin{tabular}{|c|c|c|c|c|c|}
\hline Oxide & $\begin{array}{l}\text { Waste } \\
\text { wt\% Oxide }\end{array}$ & $\begin{array}{l}\text { Normal ized } \\
\text { Waste Nor., } \\
\text { wt\% Oxide } \\
\end{array}$ & $\begin{array}{l}\text { Substituted } \\
\text { Frit, } \\
\text { wt\% Oxide } \\
\end{array}$ & $\begin{array}{c}\text { HW-39-1 } \\
\text { Glass, } \\
\text { wt\% Oxide } \\
\end{array}$ & \\
\hline $\mathrm{SiO}_{2}$ & 2.9 & 3.0 & 67.25 & 51.3 & \\
\hline $\mathrm{B}_{2} \mathrm{O}_{3}$ & 0.0 & 0.0 & 12.75 & 9.6 & \\
\hline $\mathrm{Na}_{2} \mathrm{O}$ & 10.5 & 10.7 & 10.25 & 10.4 & $\nu$ \\
\hline $\mathrm{Li}_{2} \mathrm{O}$ & 0.0 & 0.0 & 5 & 3.8 & \\
\hline $\mathrm{CaO}$ & 0.3 & 0.3 & 3.75 & 2.9 & \\
\hline $\mathrm{MgO}$ & 0.2 & 0.3 & 1 & 0.8 & \\
\hline $\mathrm{Fe}_{2} \mathrm{O}_{3}$ & 44.0 & 44.4 & & 11.1 & $\cdot$ \\
\hline $\mathrm{Al}_{2} \mathrm{O}_{3}$ & 17.0 & 17.2 & & 4.3 & \\
\hline $\mathrm{Cr}_{2} \mathrm{O}_{3}$ & 5.3 & 5.3 & & 1.3 & $\cdot$ \\
\hline $\mathrm{ZrO}_{2}$ & 2.3 & 2.4 & & 0.6 & \\
\hline $\mathrm{NiO}$ & 2.3 & 2.4 & & 0.6 & \\
\hline $\mathrm{La}_{2} \mathrm{O}_{3}$ & 2.2 & 2.2 & & 0.5 & \\
\hline $\mathrm{SO}_{4}$ & 1.8 & 1.8 & & 0.4 & \\
\hline $\mathrm{Nd}_{2} \mathrm{O}_{3}$ & 1.7 & 2.1 & & 0.5 & \\
\hline $\mathrm{MoO}_{3}$ & 1.2 & 1.2 & & 0.3 & \\
\hline$F$ & 1.2 & 1.2 & & 0.3 & \\
\hline CuO & 0.6 & 0.6 & & 0.1 & \\
\hline TOC & 0.6 & 0.6 & & & \\
\hline $\mathrm{MnO}_{2}$ & 0.6 & 0.7 & & 0.2 & \\
\hline $\mathrm{CeO}_{2}$ & 0.6 & 0.7 & & 0.2 & \\
\hline $\mathrm{RuO}_{2}$ & 0.6 & 0.6 & & 0.1 & \\
\hline $\mathrm{U}_{3} \mathrm{O}_{8}$ & 0.6 & SUB Nd & & & \\
\hline $\mathrm{Cs}_{2} \mathrm{O}$ & 6.0 & 1.0 & & 0.2 & $\cdot$ \\
\hline $\mathrm{BaO}$ & 0.4 & 0.4 & & 0.1 & 9 \\
\hline Sro & 0.4 & 0.4 & & 0.1 & \\
\hline $\operatorname{Pr}_{6} 0_{11}$ & 0.4 & 0.4 & & 0.1 & : \\
\hline $\mathrm{Tc}_{2} \mathrm{O}_{7}$ & 0.4 & SUB Mn & & & \\
\hline $\mathrm{Rb}_{2} \mathrm{O}$ & 0.2 & SUB CS & & & \\
\hline $\mathrm{Y}_{2} \mathrm{O}_{3}$ & 0.2 & 0.2 & & 0.04 & \\
\hline
\end{tabular}


TABLE 2.1. (contd)

\begin{tabular}{|c|c|c|c|c|}
\hline Oxide & 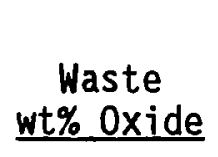 & $\begin{array}{l}\text { Normal ized } \\
\text { Waste Nor., } \\
\text { wt\% Oxide }\end{array}$ & $\begin{array}{c}\text { Substituted } \\
\text { Frit, } \\
\text { wt\% Oxide } \\
\end{array}$ & $\begin{array}{r}\text { HW-39-1 } \\
\text { Glass, } \\
\text { wt\% Oxide } \\
\end{array}$ \\
\hline $\mathrm{Sm}_{2} \mathrm{O}_{3}$ & 0.2 & 0.2 & & 0.04 \\
\hline Pdo & 0.2 & DEL & & \\
\hline $\mathrm{Rh}_{2} \mathrm{O}_{3}$ & 0.2 & DEL & & \\
\hline $\mathrm{NpO}_{2}$ & 0.1 & SUB Ce & & \\
\hline $\mathrm{TeO}_{2}$ & 0.1 & DEL & & \\
\hline $\mathrm{Pm}_{2} \mathrm{O}_{3}$ & 0.1 & SUB Md & & \\
\hline Be0 & 0.1 & SUB Mg & & \\
\hline $\mathrm{SeO}_{2}$ & 0.03 & DEL & & \\
\hline $\mathrm{SnO}_{2}$ & 0.02 & DEL & & \\
\hline Cdo & 0.02 & DEL & & \\
\hline $\mathrm{Eu}_{2} \mathrm{O}_{3}$ & 0.02 & SUB Nd & & \\
\hline $\mathrm{PuO}_{2}$ & 0.02 & SUB Ce & & \\
\hline $\mathrm{Am}_{2} \mathrm{O}_{3}$ & 0.02 & SUB Nd & & \\
\hline $\mathrm{P}_{2} \mathrm{O}_{5}$ & 0.02 & DEL & & \\
\hline $\mathrm{Ag}_{2} \mathrm{O}$ & 0.01 & DEL & & \\
\hline $\mathrm{Nb}_{2} \mathrm{O}_{5}$ & 0.01 & SUB Mo & & \\
\hline $\mathrm{Gd}_{2} \mathrm{O}_{3}$ & 0.01 & 0.01 & & 0.003 \\
\hline $\mathrm{Ta}_{2} \mathrm{O}_{5}$ & 0.01 & DEL & & \\
\hline \multirow[t]{2}{*}{$\mathrm{TiO}_{2}$} & 0.01 & DEL & & \\
\hline & $\overline{100.00}$ & $\overline{100.00}$ & $\overline{100.00}$ & $\overline{100.00}$ \\
\hline
\end{tabular}

\subsection{IEST MATRIX}

Leach testing over a range of $(S A / V) \cdot t$ values was proposed as a means of simulating a wide range of concentration conditions. MCC -1 and MCC -3 . tests were conducted over a range of $(S A / V) \cdot t$ values to evaluate the leaching behavior of the HW-39-1 reference glass. These ranges were chosen to provide overlapping values of $(S A / V) \cdot t$. The MCC -1 tests (monolith) provided data in the lower range of $(S A / V) \cdot t$ that was used to determine the forward glass dissolution rate $\left(k_{+}\right)$, which is the rate at which the glass dissolves when is first comes in contact with the leachant. The MCC-3 (powder) testing 
provided data to corroborate a portion of the MCC-1 results and to extend the $(S A / V) \cdot t$ values into the saturation/solubility controlled portion of the dissolution curve. All MCC-3 tests were conducted using 100 to 200 mesh-size powders.

The test matrix given in Table 2.2 shows the number of replicates and blanks at each time and $S A / V$ ratio. The overlap of $\log [(S A / V) \cdot t]$ values provided by the test matrix is shown in Figure 2.1. All tests were conducted in Teflon PFA leach containers cleaned according to MCC -1 procedures. The temperatures within the ovens where testing was conducted were maintained at $90 \pm 1{ }^{\circ} \mathrm{C}$. Oven temperatures were continuously monitored with cal ibrated thermistors and recorded throughout the tests.

TABLE 2.2. Leaching Test Matrix for HWVP Waste Form Qualification. Each value in the matrix indicates the number of replicates.

Test Duration In Days

\begin{tabular}{|c|c|c|c|c|c|c|c|c|c|c|c|}
\hline Test & 0.25 & $\underline{0.5}$ & $\underline{1}$ & $\underline{3}$ & $\underline{7}$ & 14 & $\underline{28}$ & $\underline{56}$ & $\underline{91}$ & 182 & $\underline{364}$ \\
\hline MCC -1 & Temp $=90^{\circ} \mathrm{C}$ & & & & & & & & & & \\
\hline$S A / V=10 m^{-1}$ & 3 & 3 & 3 & 3 & 3 & 3 & 3 & 3 & 3 & 6 & 6 \\
\hline Blanks (a) & 1 & 1 & 1 & 1 & 1 & 1 & 1 & 1 & 1 & 1 & 1 \\
\hline \multicolumn{12}{|l|}{ MCC -3} \\
\hline$S A / V=530 \mathrm{~m}^{-1}$ & 3 & 3 & 3 & 3 & 3 & 3 & 3 & 3 & 3 & 6 & 6 \\
\hline $\mathrm{Bl}$ anks (a) & 1 & 1 & 1 & 1 & 1 & 1 & 1 & 1 & 1 & 1 & \\
\hline
\end{tabular}

MCC -3

$\begin{array}{llllllllllll}S A / V=2000 m^{-1} & 3 & 3 & 3 & 3 & 3 & 3 & 3 & 3 & 3 & 6 & 6 \\ B T \text { anks }(a) & 1 & 1 & 1 & 1 & 1 & 1 & 1 & 1 & 1 & 1 & 1\end{array}$

MCC -3

$\begin{array}{lllllllll}\mathrm{SA} / \mathrm{V}=20000 \mathrm{~m}^{-1} & 3 & 3 & 3 & 3 & 3 & 3 & 3 & 3 \\ \mathrm{Bl} \text { anks }(\mathrm{a}) & 1 & 1 & 1 & 1 & 1 & 1 & 1 & 1\end{array}$

(a) Blanks are leaching containers with leachant subjected to test conditions with no glass present. 


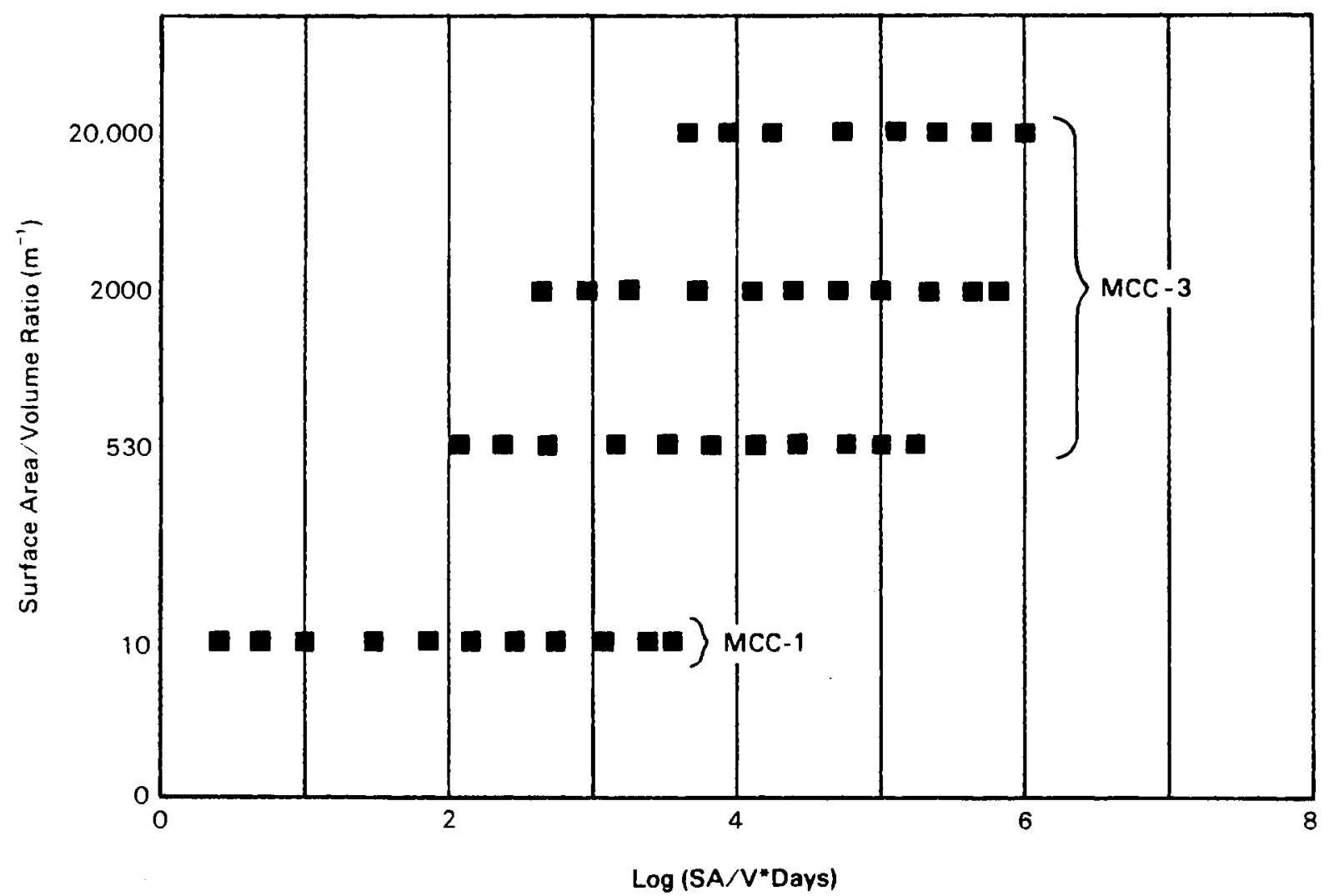

FIGURE 2.1. Resultant $\log [(S A / V) \cdot t]$ Values for Each Type of Test in the Test Matrix. Note the overlap of $\log [(S A / V) \cdot t]$ values.

All of the MCC-1 testing was conducted at an SA/V value of $10 \mathrm{~m}^{-1}$. By adjusting the proportions of glass powder and the amount of leachant in the MCC -3 tests, testing was conducted at higher SA/V values. The powders for all MCC-3 testing were sieved, using nylon screens, to retain the -100 -mesh $(149 \mu \mathrm{m})+200$-mesh $(74 \mu \mathrm{m})$ particle sizes. The glass surface area per gram of glass powder was calculated to be $2 \times 10^{-2} \mathrm{~m}^{2} / \mathrm{g}$ for $-100+200$ mesh $\mathrm{HW}-39-1$ glass powder.

The amount of leachants and powdered glass in the leaching vessel used to produce the required $S A / V$ ratios is presented in Table 2.3. Three replicate experiments were performed at $90^{\circ} \mathrm{C}$ at each of 36 combinations of SA/ $\mathrm{V}$ ratio and time. Replicate tests were conducted at the same time within the same ovens. Leachates were submitted for elemental analysis by inductively coupled plasma atomic emission spectroscopy (ICP) with one analysis per leachate. Leachates from replicate tests were analyzed sequentialiy one 
IABLE 2.3. Glass and Leachate Amounts used to Produce Required SA/V Ratios in MCC-3 Testing

\begin{tabular}{|c|c|c|}
\hline$\underline{S A / V, m^{-1}}$ & Glass, $g$ & Leachate, $\mathrm{mL}$ \\
\hline 530 & 1.067 & 40 \\
\hline 2,000 & 4.0 & 40 \\
\hline 20,000 & 10.00 & 10 \\
\hline
\end{tabular}

after another. A blank was also run at each $S A / V$ ratio and time combination. The $S A / V$ ratios investigated were $10 \mathrm{~m}^{-1}, 530 \mathrm{~m}^{-1}, 2,000 \mathrm{~m}^{-1}$, and $20,000 \mathrm{~m}^{-1}$; the times ranged from 0.25 days to 364 days. The product $(S A / V) \cdot t$ ranged from 2.5 days $/ \mathrm{m}$ to 560,000 days $/ \mathrm{m}$. The blanks (leach vessels with leachant but no glass) were used to determine if sources of contamination (in the leachant or from the leach vessels) were present.

At the completion of each test, the $\mathrm{pH}$ of the solutions were measured and the solutions were filtered sequentially with $0.45 \mu \mathrm{m}$ and 18 angstrom (A) filters. The filtered solutions were then analyzed for total concentrations of $g l$ ass constituents, which were corrected by subtracting concentrations from the blank runs. All analyses were done at room temperature $\left(25^{\circ} \mathrm{C}\right)$. The chemical analyses for dissolved cations were conducted using inductively coupled plasma atomic emission spectroscopy (ICP). Cesium concentrations were analyzed by atomic absorption (AA). No anion analysis was conducted.

\subsection{QUALITY ASSURANCE}

A11 testing was conducted under QA requirements commensurate with other licensing-related programs conducted at PNL. Testing was conducted such that all instrument calibrations and materials certification were traceable, test procedures and associated training activities documented, and compliance of test methods and results could be compared with future $Q A$ requirements. 


\subsection{RESULTS}

The blank corrected elemental concentrations and normalized concentrations for the $0.45 \mathrm{~m}$ and $18 \AA$ leachant for each test are summarized in Appendix A. Normalized elemental concentrations were calculated using the following equation:

$$
N C_{i j k}=\frac{C_{i j k}-\bar{B}_{i}}{\bar{f}_{j}}
$$

where $N C_{i j k}=$ normalized concentration of element $i\left(g / \mathrm{m}^{3}\right.$ or $\left.\mathrm{ppm}\right)$ from the $j$ th specimen (replicate) of the $k$ th TEST (NOMINAL SA/V AND DURATION OF THE TEST).

$C_{i j k}=$ concentration of element $i$ in leachate $\left(\mathrm{g} / \mathrm{m}^{3}\right.$ or $\left.\mathrm{ppm}\right)$

$\bar{B}_{i}$. = concentration of element $i$ in blank $\left(g / \mathrm{m}^{3}\right.$ or $\left.\mathrm{ppm}\right)$

$\bar{f}_{j} .=$ average weight fraction of element $i$ in glass ( $g$ of element $i$ per $\mathrm{g}$ of glass).

Normalized concentrations provide a method of evaluating the congruency of the glass dissolution (i.e., whether elements in the glass matrix dissolve uniformly). The normalized concentrations of each element will be the same if the dissolution is truly congruent and the concentration of the component is below its solubility limit in solution. The normalized concentrations also provide for comparing the dissolution rates of glasses with different compositions.

Figures 3.1 through 3.4 each display a number of curves of normalized concentrations of $\mathrm{Li}, \mathrm{B}, \mathrm{Cs}, \mathrm{Ca}, \mathrm{Si}, \mathrm{Al}, \mathrm{Na}$, and $\mathrm{Sr}(0.45 \mu \mathrm{m}$ filtered) versus $(S A / V) \cdot t$ values. Figure 3.1 represents the results for the MCC -1 tests with $S A / V$ ratios of $10 \mathrm{~m}^{-1}$. Figures $3.2,3.3$, and 3.4 display the results for the MCC -3 tests with $S A / V$ ratios of $530,2,000$, and $20,000 \mathrm{~m}^{-1}$, respectively. Figures 3.5 and 3.6 show normalized $B$ and $S i$ concentrations, respectively, over the complete range of $(S A / V) \cdot t$ values tested. 


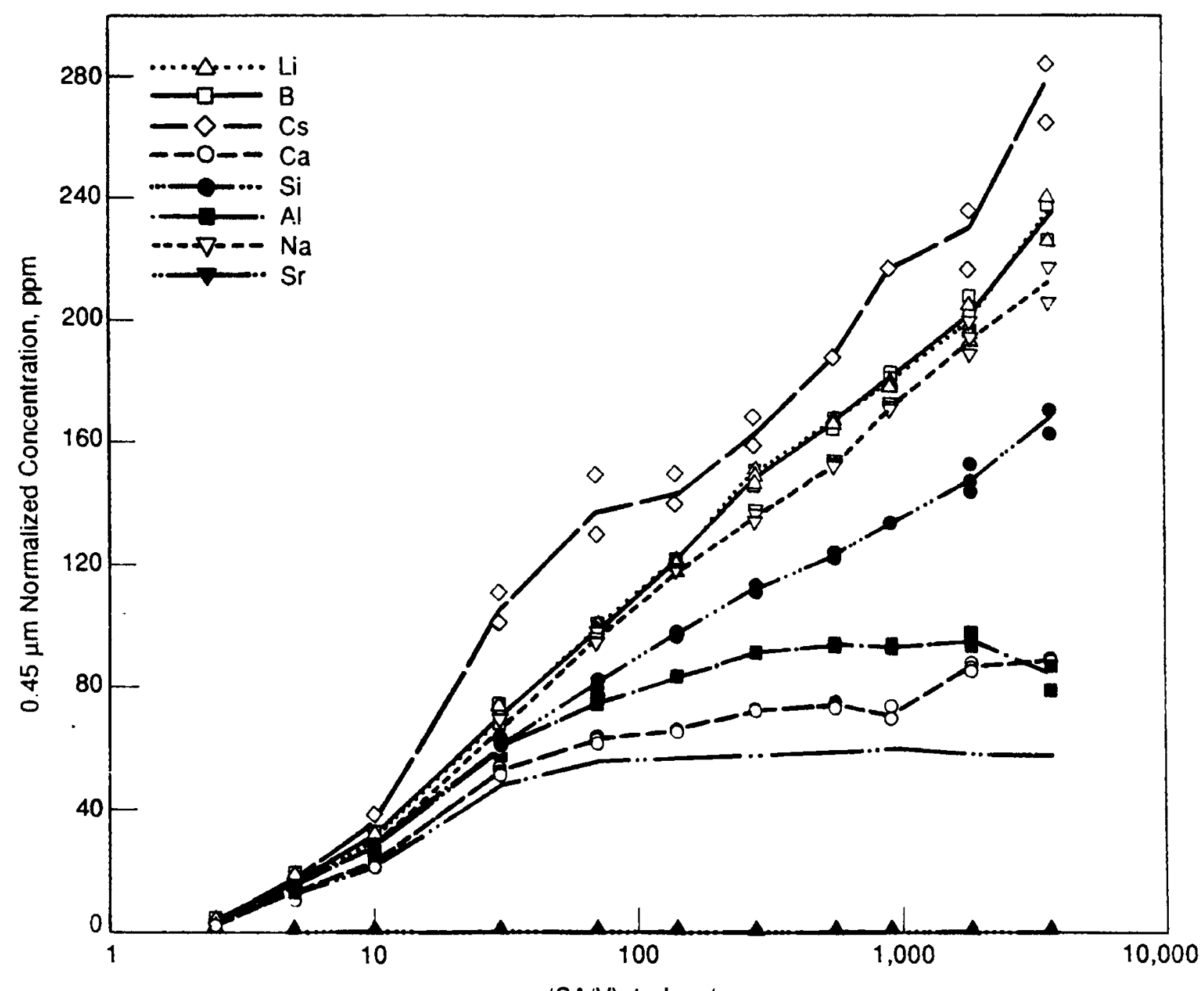

(SAV) $\cdot \mathrm{t}$, days/m

FIGURE 3.1. Plot of Normalized Concentrations Versus $(S A / V) \cdot t$ from MCC-1 Leach Tests of HW-39-1 at an SA/V of $10 \mathrm{~m}^{-1}$

There are discontinuities in the normalized concentrations of all constituents as a function of $(S A / V) \cdot t$ when considering data at different $S A / V$ ratios. The discontinuities are apparent for $S i$ and $B$ in Figures 3.5 and 3.6, respectively, which show the $0.45 \mu \mathrm{m}$ filtered data for these constituents on a $\log -\log$ scale. In going from one SA/ $V$ ratio to another, both the concentrations and the rates of change of the concentrations exhibit discontinuities. The discontinuity in going from an SA/ $V$ ratio of 2,000 to $20,000 \mathrm{~m}^{-1}$ is especially obvious in Figures 3.5 and 3.6. The discontinuities would be even more apparent if the data were not plotted on a log scale. In 


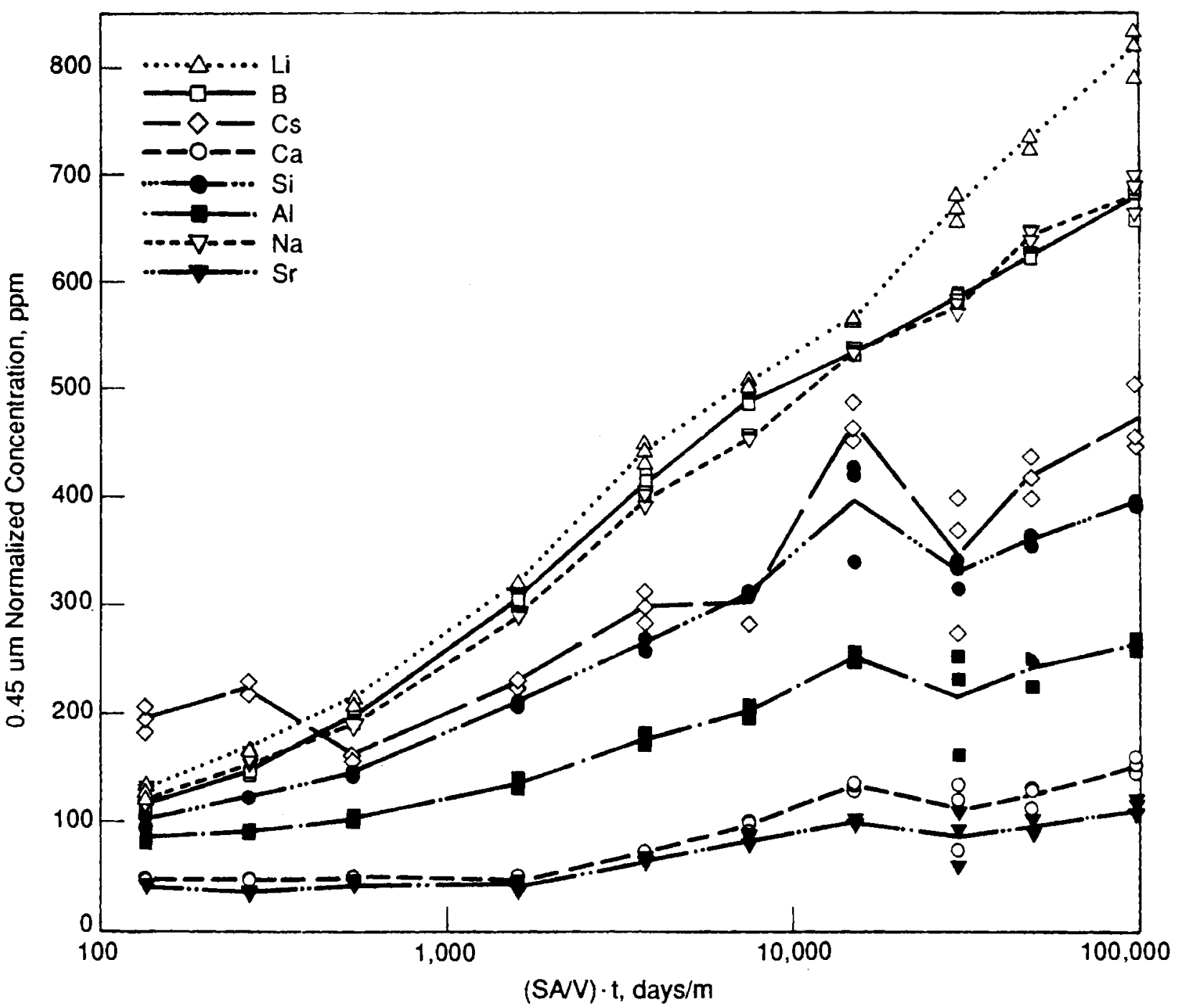

FIGURE 3.2. PIot of Normalized Concentrations Versus $(S A / V) \cdot t$ from MCC-3 Leach Tests of HW-39-1 at an SA/V of $530 \mathrm{~m}^{-1}$

Figures 3.5 and 3.6, it is apparent that the data for any given $S A / V$ ratio follow a reasonably consistent trend, but data for different SA $\mathrm{V}$ ratios follow different trends. It remains to be demonstrated that these differences are statistically significant, although it should be emphasized that the data of Figures 3.5 and 3.6 represent the average of three experimental data points, and in general the scatter between replicates was very small relative to the differences between the data sets. However, the replicates contain only some of the sources of variation that need to be considered in comparing the results for different SA/V values. 


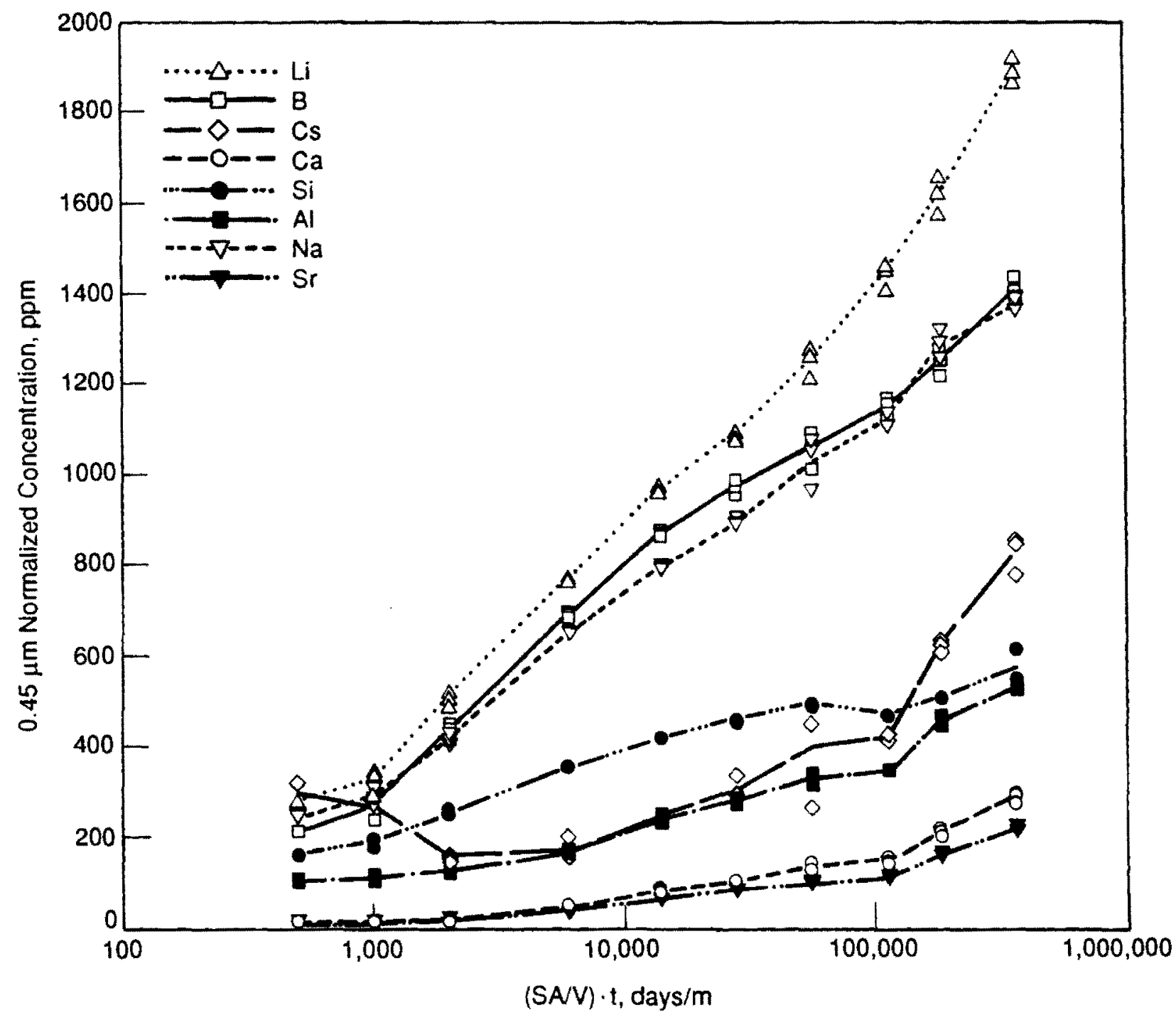

FIGURE 3.3. Plot of Normalized Concentrations Versus (SA/Y) -t from MCC-3 Leach Tests of HW-39-1 at an SA/V of $2,000 \mathrm{~m}^{-1}$ 


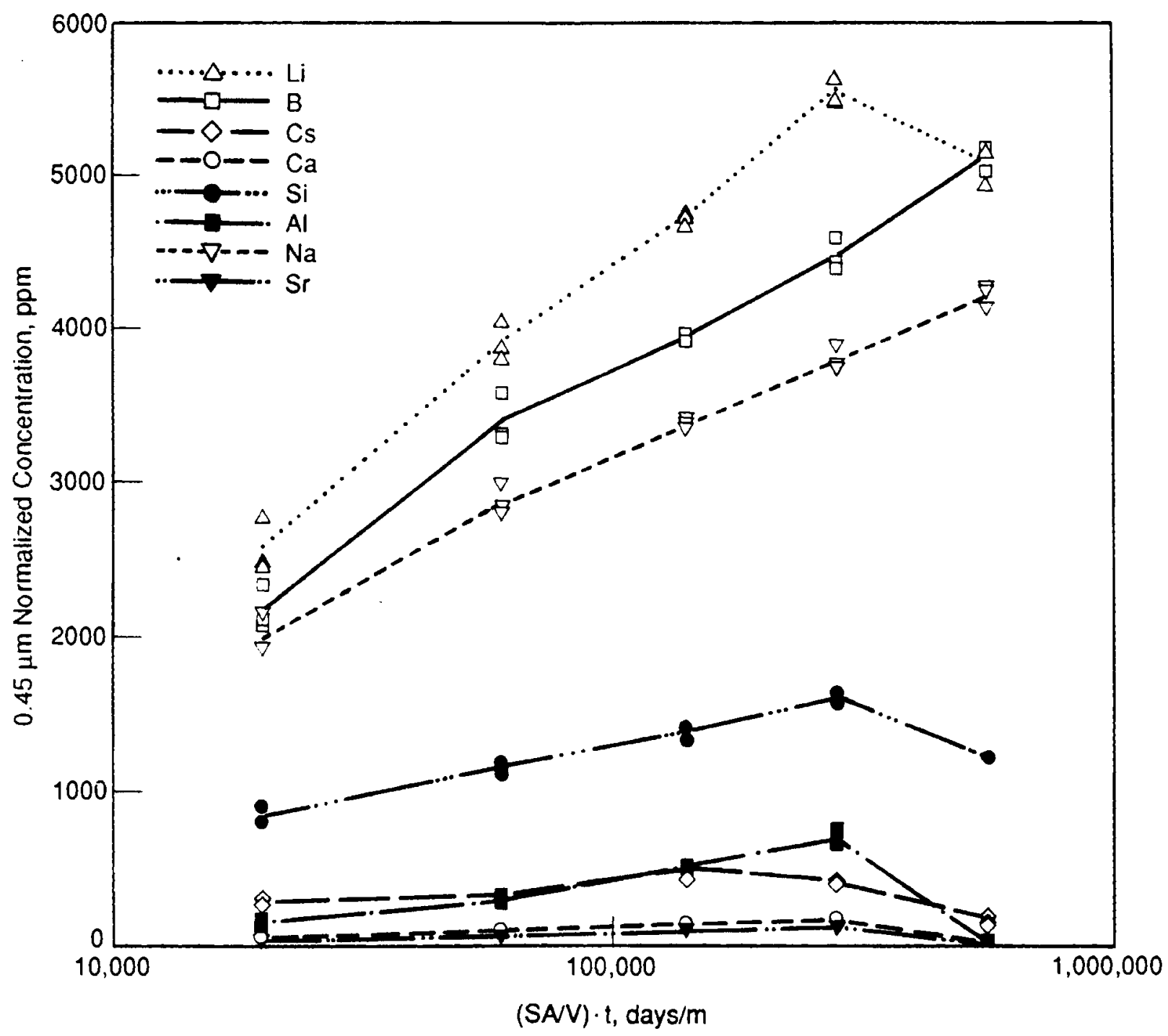

FIGURE 3.4. Plot of Normalized Concentrations Versus $(S A / V) \cdot t$ from $M C C-3$ Leach Tests of $\mathrm{HW}-39-1$ at an SA/V of $20,000 \mathrm{~m}^{-1}$ 


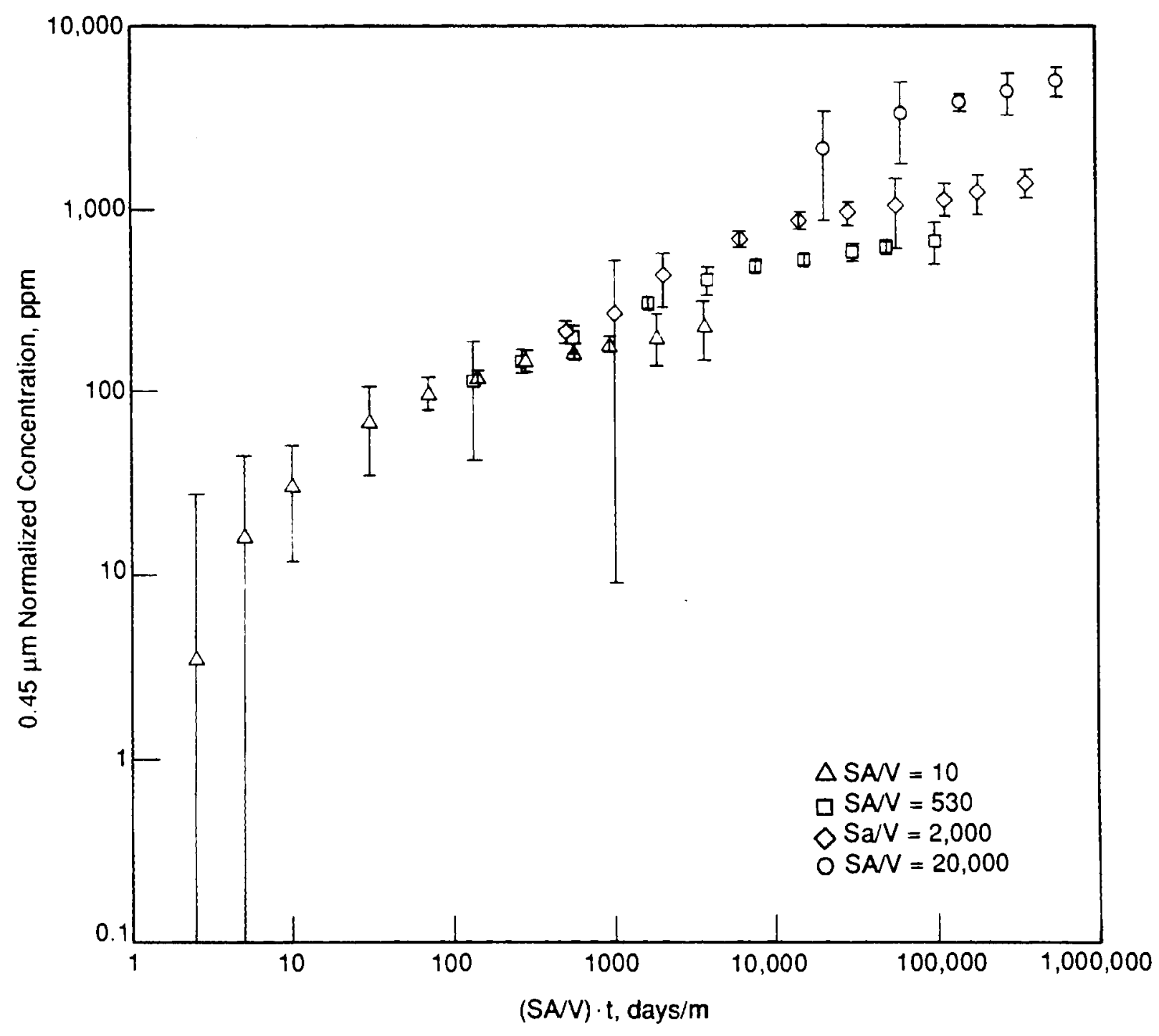

FIGURE 3.5. Plot of Normalized Boron Concentrations Versus (SA/V) -t from HW-39-1 Leach Tests. Error bars represent 95\%/95\% tolerance interval on individual normalized concentration values for each $(S A / V) \cdot t$. 


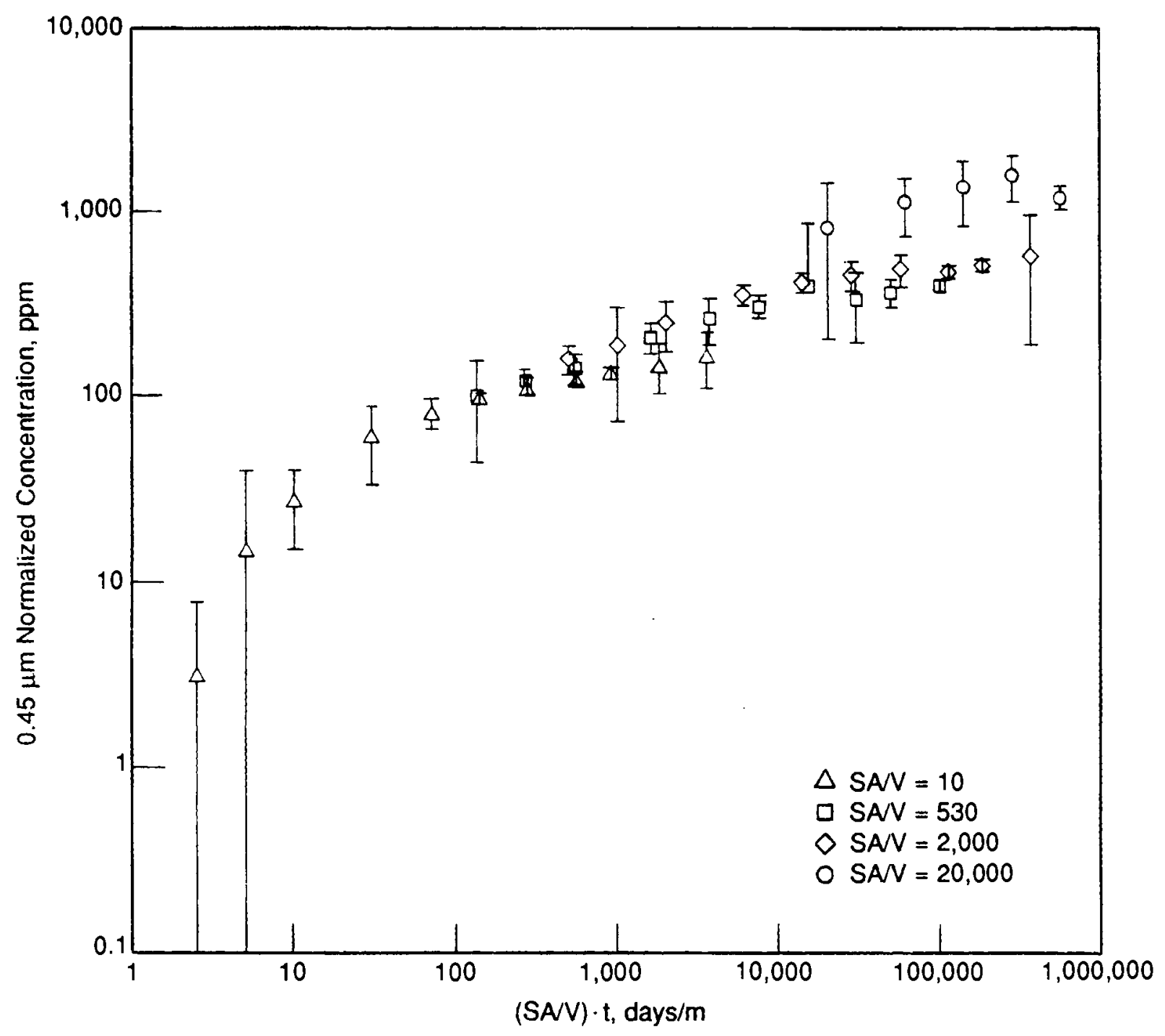

FIGURE 3.6. Plot of Normalized Silicon Concentration Versus (SA/V) t from HW-39-1 Leach Tests. Error bars represent 95\%/95\% tolerance interval on individual normalized concentration values for each $(S A / V) \cdot t$. 


\subsection{DISCUSSION}

Five topics are discussed in the following section based on the reported results. First, the suitability of $(S A / V) \cdot t$ scaling to predict the dissolution behavior of glasses at long times is discussed. Second, the data are used to show the congruent (uniform) dissolution of the glass before leachant saturation. Third, the forward and steady-state dissolution rates of the HW-39-1 glass are estimated. Fourth, the test data and testing methodology are statistically evaluated. Finally, recommendations for changes to the testing methodology are made.

\section{$4.1 \quad(S A / V) \cdot t$ SCALING}

The $(S A / V) \cdot t$ scaling method uses high-SA/V, short-time (less than one year) data to develop and test a glass dissolution rate law that could be used to estimate lower-SA $\mathrm{V}$, long-time (100 yeart) data that are impractical to obtain. This method is also often used to estimate steady-state glass dissolution rates.

Figures 3.5 and 3.6 show that normalized $B$ and $S i$ concentrations at equivalent $(S A / V) \cdot t$ values increase as the $S A / V$ ratio of the leach test increases. The observed concentration differences are small (at the same $(S A / V) \cdot t$ values) for the lower-SA/V value tests compared with differences observed at the highest $S A / V$ value $\left(20,000 \mathrm{~m}^{-1}\right)$ tests. Therefore, scaling technique appears to be adequate up to an $S A / \mathrm{V}$ value of $2,000 \mathrm{~m}^{-1}$ but fails when the $S A / V$ value is increased to $20,000 \mathrm{~m}^{-1}$. The discontinuities in the data call into question the practice of equating normalized concentration data obtained at a specific $(S A / V) \cdot t$ value to equivalent values obtained using much greater experimental $S A / V$ ratios.

Figure 4.1 displays the final $\mathrm{pH}$ values of the leachates versus $(S A / V) \cdot t$, and exhibits the same pattern observed for normalized $B$ concentrations. The increase in $\mathrm{pH}$ may explain the offset in $B$ concentrations at equivalent $(S A / V) \cdot t$ values, but the reason for the different $\mathrm{pH}$ is not evident. Different steady-state $\mathrm{pH}$ values are also observed for each set of $\mathrm{SA} / \mathrm{V}$ tests. Although the initial rate of $\mathrm{pH}$ increase can be determined for 


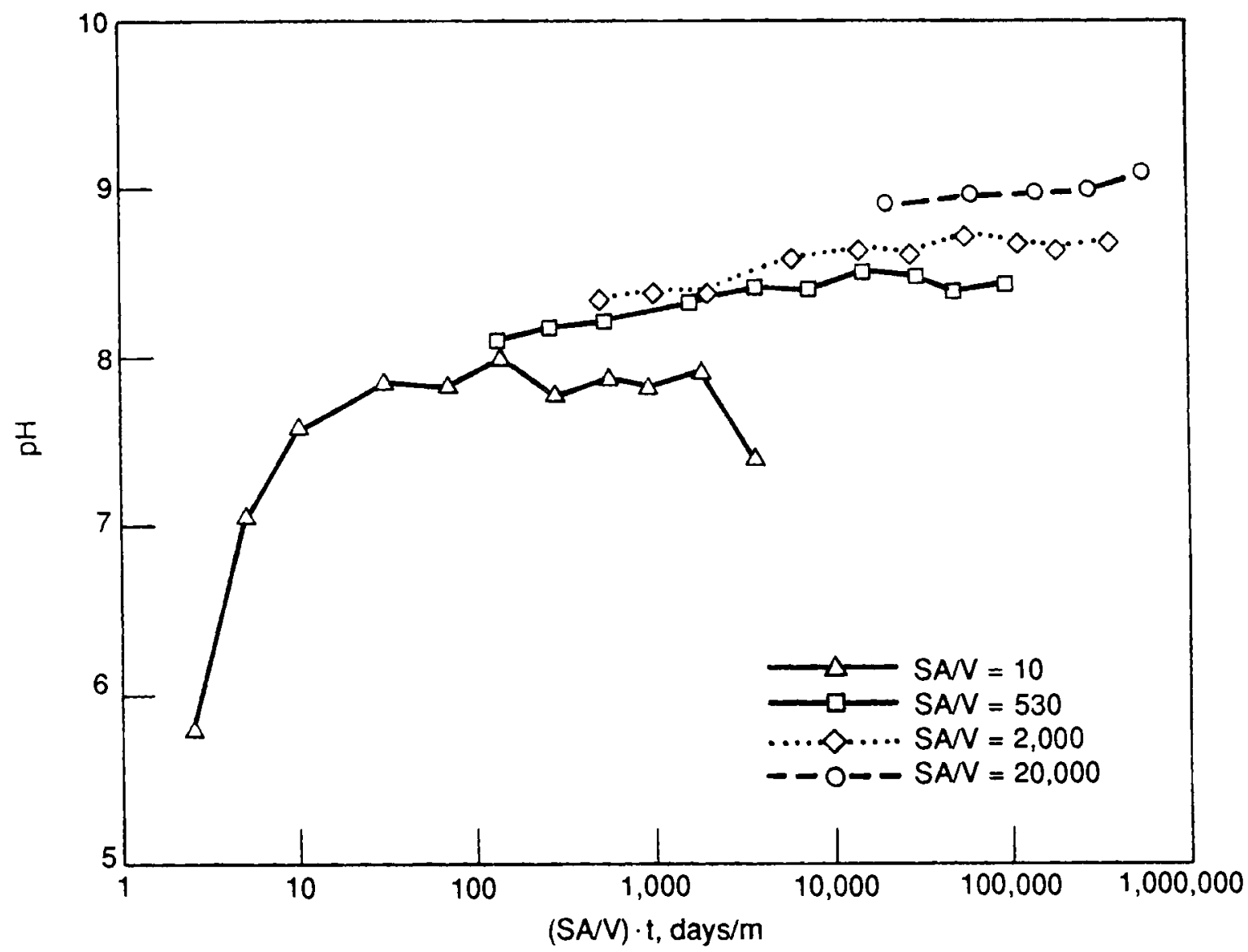

FIGURE 4.1. Plot of Calculated $90^{\circ} \mathrm{C} \mathrm{pH}$ Values Versus $(S A / V) \cdot t$ from HW-39-1 Leach Tests. $90^{\circ} \mathrm{C} \mathrm{pH}$ values were calculated from $\mathrm{pH}^{\prime} \mathrm{s}$ measured at $25^{\circ} \mathrm{C}$.

the lowest $S A / V$ ratios (the MCC-1 tests), the rate cannot be determined for the higher $S A / V$ ratios. The initial rise in $\mathrm{pH}$ at the higher $S A / V$ ratios occurred before the first samples were taken. This initial pH increase has al so been observed in other tests. (8) The effects of $\mathrm{pH}$ on elemental leach rates from glasses are well documented. Borosilicate waste glasses exhibit a decrease in durability as the $\mathrm{pH}$ approaches values of 10 or higher. (9) Thus, higher $B$ concentrations are expected at the higher $\mathrm{pH}$ values observed at higher SA/V values. Under $\mathrm{pH}$ buffered conditions, such as naturally buffered groundwaters, rapid increases in $\mathrm{pH}$ may not be a concern. Scaling by 
$(S A / V) \cdot t$ (using $S A / V$ values of greater than 2000) may provide an accurate method for studying steady-state glass dissolution behavior in buffered solutions.

\subsection{CONGRUENT DISSOLUTION}

Congruent (uniform) dissolution of the glass is an assumption made in the modeling of glass dissolution. The dissolution congruency of the HWVP reference glass can be assessed by plotting the normalized concentrations of the glass constituents in the solutions at early (SA/V) - t. If dissolution is congruent, the normalized concentrations of all constituents should be the same at lower $(S A / V) \cdot t$ values. At higher $(S A / V) \cdot t$ values, however, some of the constituents may precipitate, and thus their concentrations in solution will not continue to rise and may decrease as more glass dissolves. A plot of the normalized concentrations of various glass constituents as a function of $\log [(S A / V) \cdot t]$ in the tests with an $S A / V$ ratio of $10 \mathrm{~m}^{-1}$ is shown in Figure 4.2. Dissolution appears reasonably congruent up to approximately one day. Thereafter, the effects of precipitation can be observed, particularly in the 7-day data.

Another measure of congruency is to calculate the ratio of the normalized concentration of each glass constituent to the normalized concentration of $\mathrm{Si}, \mathrm{NC}_{j} / \mathrm{NC}_{\mathrm{Si}}$. If dissolution is congruent, this ratio should equal one at early $(S A / V) \cdot t$. Figure 4.3 is a plot of the ratio of the normalized concentrations of various constituents to the normalized concentration of $\mathrm{Si}$, $N C_{j} / N_{S} i$, versus $\log [(S A / V) \cdot t]$ for the data of Figure 4.2. In this plot, the data at low $(S A / V) \cdot t$ appear more scattered than in Figure 4.2 because this method of plotting the data has the effect of expanding the $y$-axis scale at lower $(S A / V) \cdot t$. Some of the offset for the deference elements for a $N C_{j} / N C_{S i}$ value of 1 is due to analytical bias and the offset remains stable out to 1 to 3 days. Dissolution still appears reasonably congruent up to at least one day. The question of congruence, however, should be objectively addressed by considering all the data (i.e., the three replicate points) and using statistical techniques to test hypotheses that the normalized concentrations of the various constituents are the same at early $(S A / V) \cdot t$. This type of analysis is recommended for future work. 


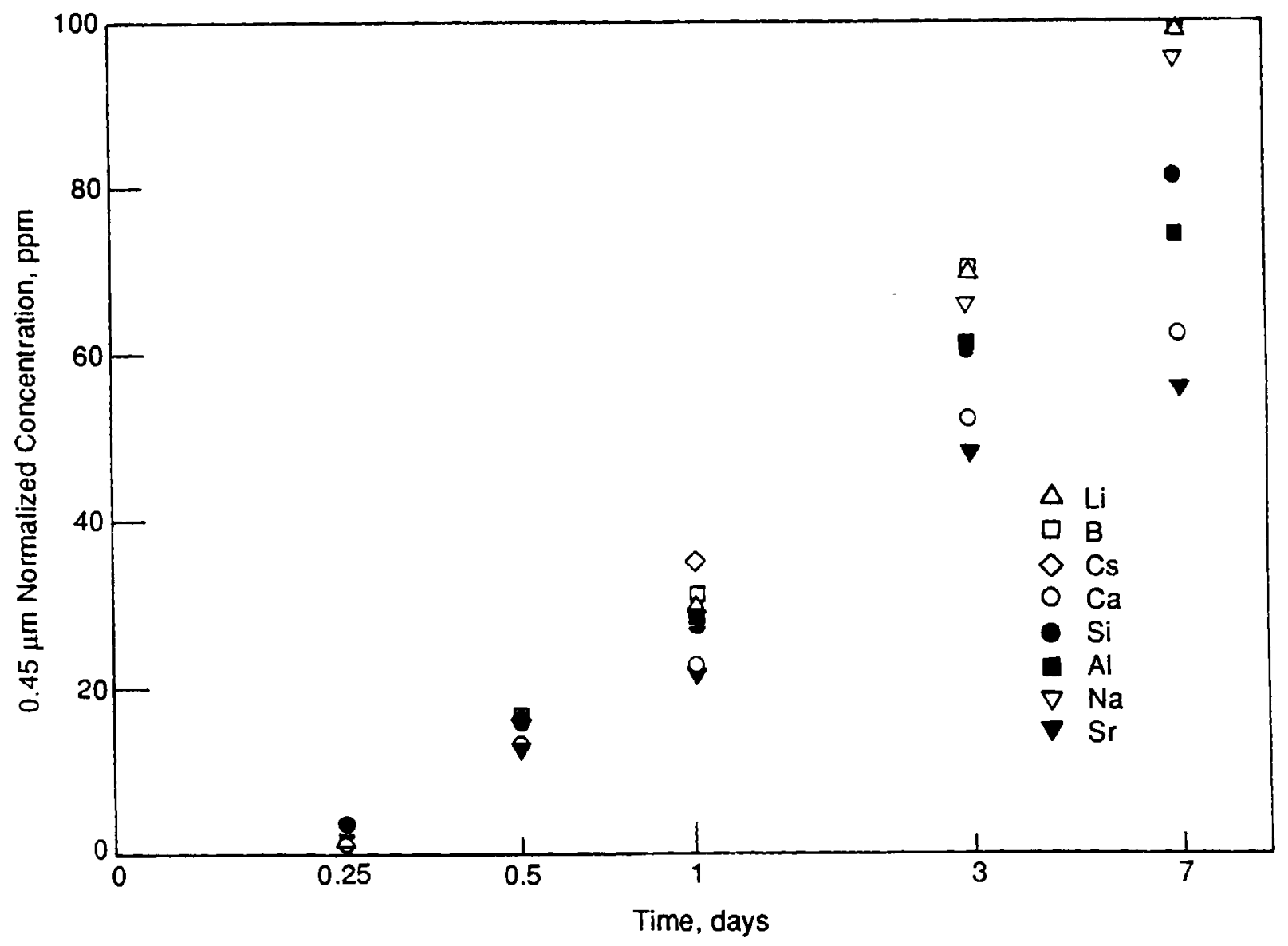

FIGURE 4.2. Normal ized Concentratjon Versus (SA/V) - t from HW-39 Leach Tests at $S A / V=10 \mathrm{~m}^{-1}, 90^{\circ} \mathrm{C}$, in Deionized Water at 0.25 , $0.5,1,3$, and 7 Days

In Figure 4.3, a systematic deviation of $\mathrm{NC}_{j} / \mathrm{NC}_{S i}$ from one (horizontal line) is apparent for $B, L i$, and $N a$ starting on the third day. This deviation is probably due to the precipitation of Si-bearing phases. It is likely that $\mathrm{Ca}$ and $\mathrm{Al}$ are also precipitating by the third day. The 0.25-day data for $\mathrm{Ca}$ and $\mathrm{Sr}$ are very close to detection limits, which may account for the lower ratios of these elements at this time. The Cs data in Figures 4.2 and 4.3 should be ignored, as it was later determined that there were large analytical errors associated with $C$.

The HWVP reference glass (HW-39) dissolves reasonably congruently in deionized water at early times. It is likely that congruent dissolution also occurs at later times, but this claim cannot be substantiated from the 


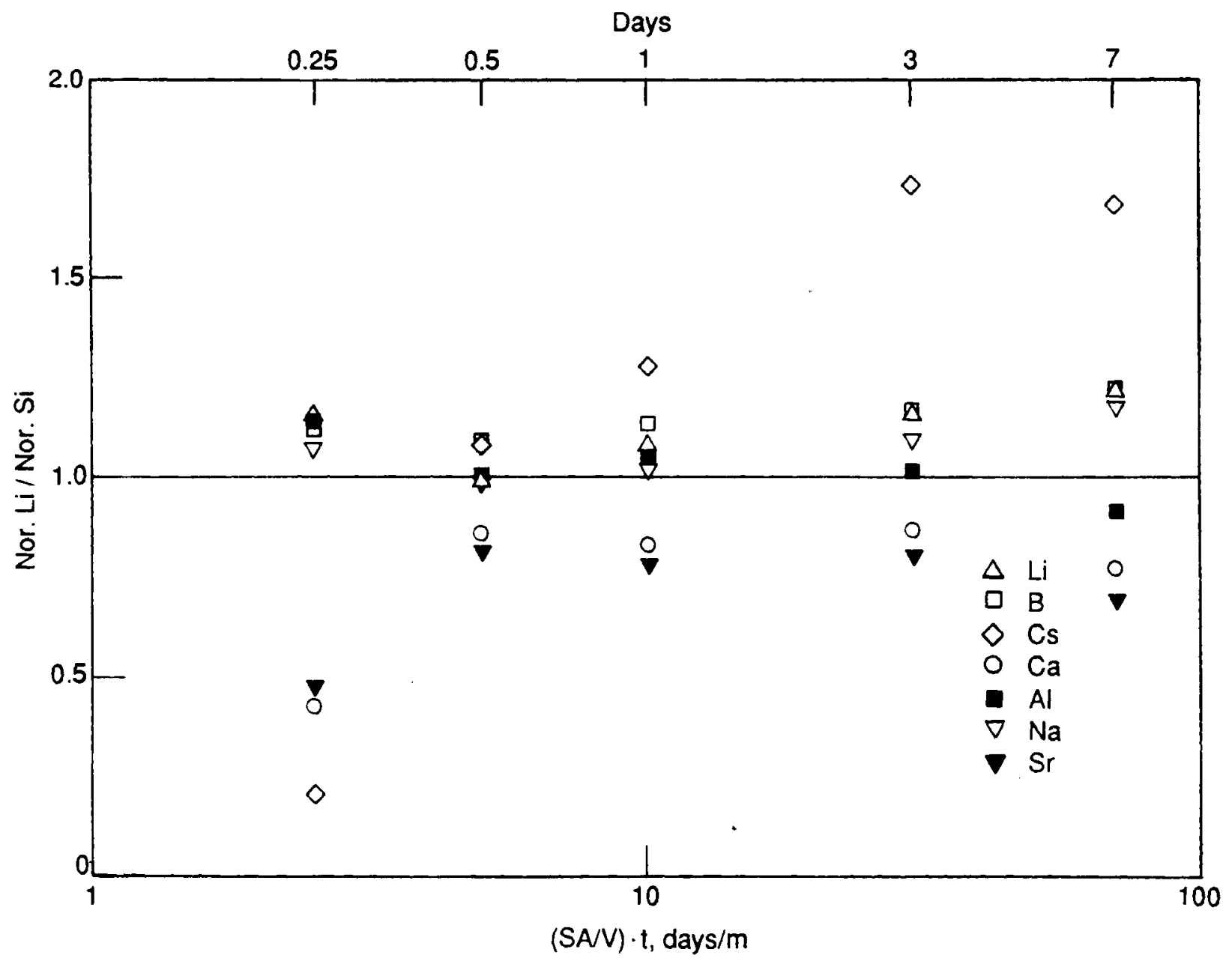

FIGURE 4.3. Ratios of the Normalized Concentration of Each Element to the Normalized Concentration of Silicon Versus $(S A / V) \cdot t$ for $S A / V=10 \mathrm{~m}^{-1}$

solution concentrations of the glass constituents because some of the constituents precipitate from solution.

\subsection{FORWARD AND STEADY-STATE DISSOLUTION RATES}

The data generated were also used to assess glass durability in the three different regimes $(10)$ typically observed in glass dissolution: 1) the period in which the glass first contacts a leachate and the glass dissolution rate is uninhibited by any solubility effects, 2) a transient regime where the increasing concentrations of dissolved glass components in the leachate slows the dissolution rate through solubility effects, and 3) a steady-state 
regime in which the dissolution rate is constant because alteration processes (saturation) have reached a steady rate.

To understand the rate of attack on the glass matrix by water, the data must be evaluated by focusing on glass elements that do not precipitate as insoluble minerals after being released from the glass matrix. Boron, a major component of most nuclear waste glasses, fits this criterion and is very soluble in aqueous environments. By evaluating $B$ release data, a good indication of the dissolution rate of the glass matrix can be obtained. The release of other glass components (including the major radionuclides) that are less soluble than B will be strongly influenced by their increasing concentrations in solution, resulting in reduced dissolution rates. Only highly soluble elements such as Tc, under oxidizing conditions, are likely to behave like B. (11) Therefore, the $B$ release rate can be utilized as a worst case estimate of release rates. The release data can be plotted as the normalized concentration of $B$ in the leachate versus $(S A / V) \cdot t$. An example of this kind of plot is shown in Figures 3.5 and 4.4. The curve plotted in Figure 4.4 can be replotted as the log of the normalized concentration versus the $\log$ of the $(S A / V) \cdot t$ product (Figure 4.5). This plot can be understood by considering what happens at the beginning of glass dissolution (no saturation effects) and under conditions where the leachate has high concentrations (near saturation) on dissolved glass or groundwater components.

At both ends of the dissolution curve, there are linear portions at which the dissolution rate is independent of time (Figure 4.4). At early times, the glass matrix dissolves with the forward dissolution rate $\left(k_{+}\right)$ because there is nothing in solution or on the surface of the glass to impede the dissolution process. The forward dissolution rate is determined by the slope of the linear portion of the curve at low saturation (smaller values of $(S A / V) \cdot t)$. After the leachate reaches saturated, steady-state conditions, the glass dissolves at a much slower constant rate $(k \infty)$. This rate is the steady-state rate in a static test and is given by the slope of the linear portion at high $(S A / V) \cdot t$ (very long times and/or high $S A / V$ in a static leach test). This steady-state rate is due to the fact that glass is a metastable state and an equilibrium condition can not be established between the solution components and the glassy phase. 


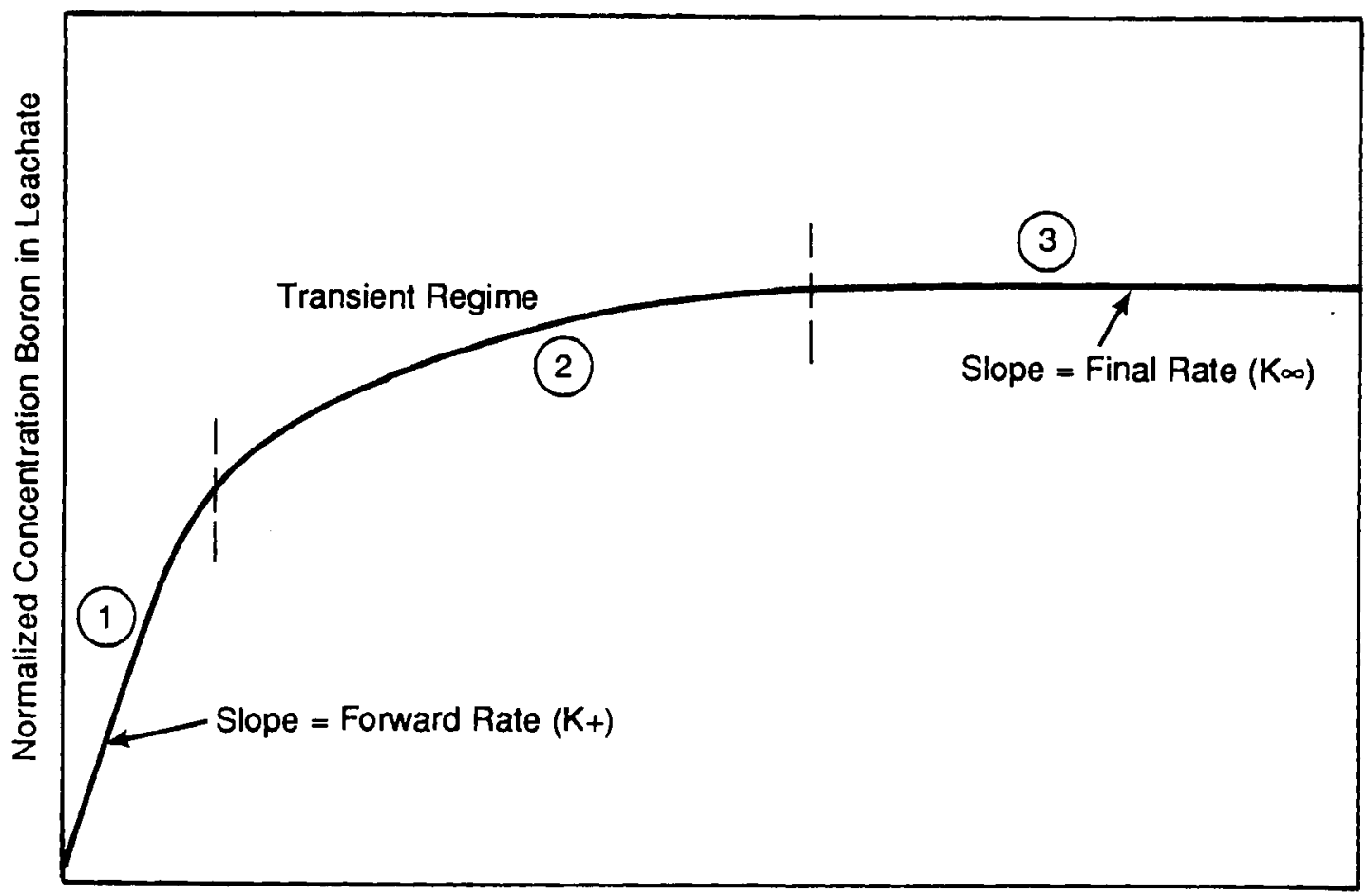

$(\mathrm{SAV} \cdot$ Time)

FIGURE 4.4. Typical Release Curve for Highly Soluble Glass Components, Such As Boron. Note the tree regimes typically observed glass dissolution: 1) unsaturated, 2) transient, and 3) saturated.

When the general formula for a straight line, $y=m x+b$, is considered, a log-log plot changes the relationship to one with unit slope. The portion of the curve in Figure 4.4 that lies between the two linear portions is a transition between the two straight lines in the log-log plot (Figure 4.5). The slopes of the two linear portions of the curve in Figure 4.4 , which correspond to the forward rate and the steady-state dissolution rate in Figure 4.5, are the intercepts of the unit slopes and the ordinate. A high value of $(S A / V) \cdot t$ in a static leach test results in solution concentrations similar to those caused by poor mass transfer in a repository. Low values of $(S A / V) \cdot t$ (and the resulting solution concentrations) are analogous to events such as shipping accidents in which the waste form is exposed to large amounts water. Data obtained over this range of $(S A / V) \cdot t$ products can be used to compare the relative chemical durability behavior of glasses in a way 


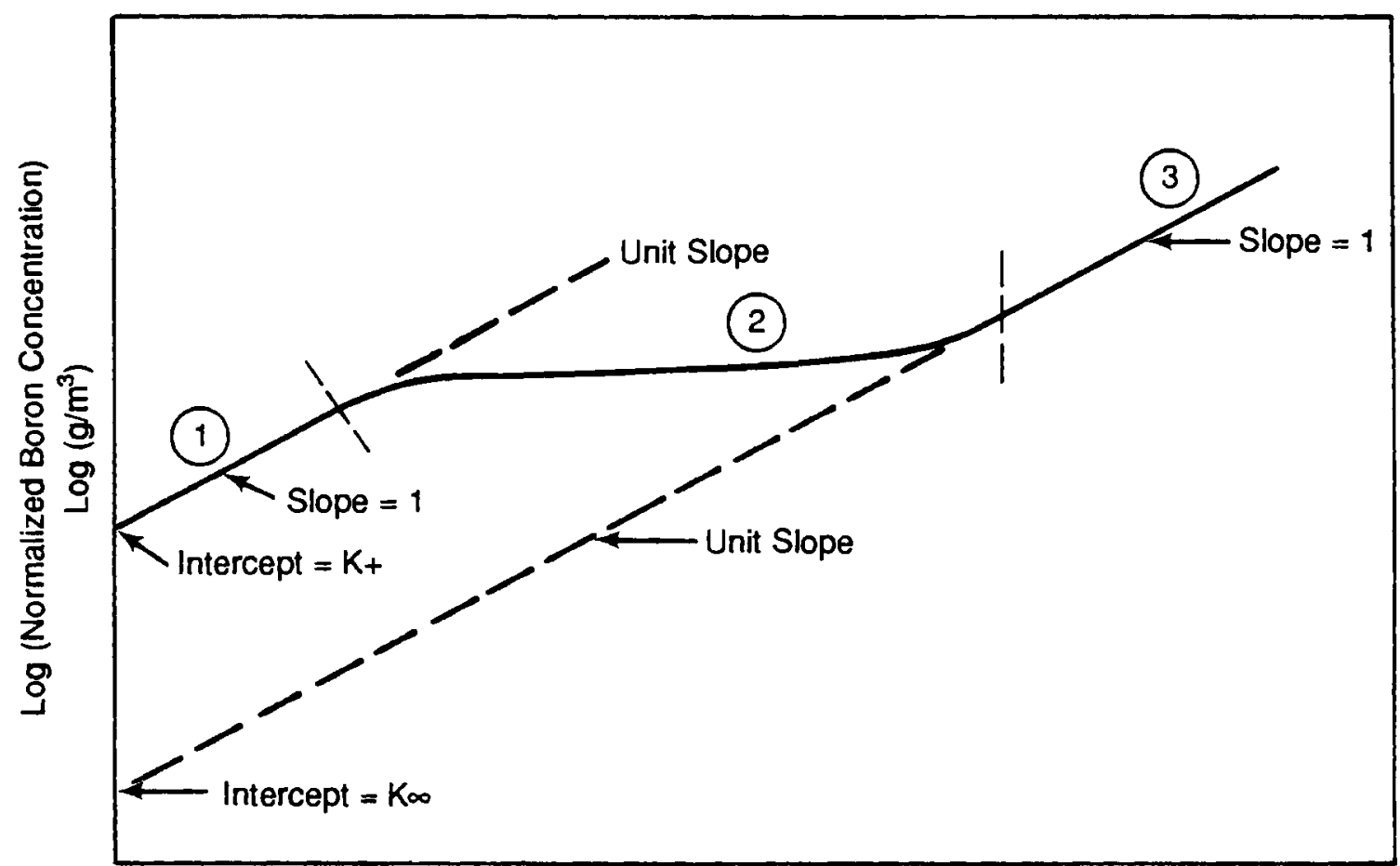

$\log (S A V \cdot$ Time $)$

FIGURE 4.5. Log-Log Plot of Typical Release Data for Highly Soluble Glass Components, Such As Boron. Note the three regimes typically observed glass dissolution: 1) unsaturated, 2) transient, and 3) saturated.

that is more relevant than a single static test condition which may be in the transient regime (Figure 4.4). The dissolution behavior of a glass at the forward and steady-state rates is important in the development of a rate law and provides a better criterion by which to compare relative long-term repository performance.

The results from the leaching tests reported herein are shown in Figure 4.6. Normalized $B$ concentration is presented as a function of $(S A / V) \cdot t$. By plotting a line of unit slope through the lower values of $S A / V \cdot t$ to the $y$ intercept, the initial rate of glass dissolution was estimated. For HW-39-1, this initial rate is approximately $1.5 \mathrm{~g} /\left(\mathrm{m}^{2} \mathrm{~d}\right)$. As the $(S A / V) \cdot t$ product increases, saturation effects begin to slow the HW-39-1 rate of dissolution. For the tests with the highest $(S A / V) \cdot t$ values, the curve had not yet returned to unit slope, indicating that the glass dissolution rates had not 


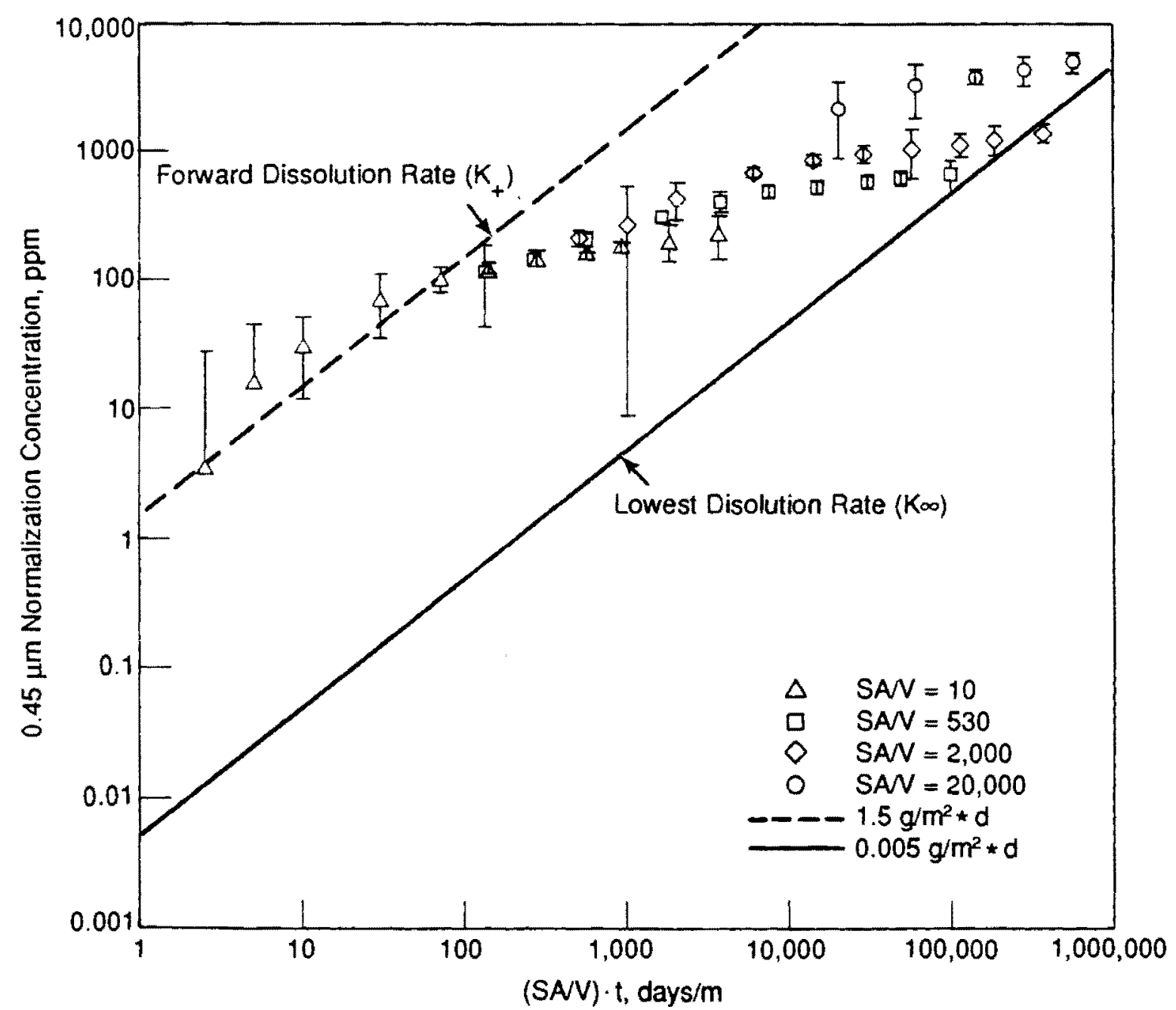

FIGURE 4.6. Normalized Boron Concentration (in Leachate) Versus (SA/V) - t for HW-39-1 with Forward and Steady-State Dissolution Rates

yet reached a steady state. Plotting a 1 ine of unit slope from the highest (SA/V) - t value tested (excluding the $20,000 \mathrm{SV} / \mathrm{A}$ data) to the $y$ intercept, a conservative estimate of the steady-state dissolution rate for HW-39-1 was determined to be $0.005 \mathrm{~g} /\left(\mathrm{m}^{2} \mathrm{~d}\right)$. At the test conditions specified in the draft WAS for qualification (MCC-1, 28-day, $90^{\circ} \mathrm{C}, 10 \mathrm{~m}^{-1}$ ), the HW-39-1 glass has a $B$ release of $0.53 \mathrm{~g} / \mathrm{m}^{2} \mathrm{~d}$, which is well below the specified $1 \mathrm{~g} / \mathrm{m}^{2} \mathrm{~d}$ limit.

It may be assumed that in the repository environment the groundwater is saturated because of slow flow rates and poor mass transfer into the 
surrounding environment. This means that the steady-state rate of glass dissolution will be reached soon after the groundwater comes in contact with the glass. Saturation of the leachant in the immediate environment will lower the release of most elements to rates below the release criterion. Only radioactive elements with high solubilities, such as Tc (under oxidizing conditions), are likely to behave like B. The solubilities of other radioactive elements are small enough to control their elemental release.

\subsection{STATISTICAL EVALUATION}

In this type of leach testing, there are four main processes subject to random or systematic errors: glass fabrication, leach testing (including specimen preparation), glass analyses, and leachate analyses. There are three "sources of variation" that might affect any of the four processes:

1. Short-Term Within-Laboratory--Variation that occurs in replicate tests or analyses conducted at the same time for a given set of conditions at a single laboratory.

2. Long-Term Within-Laboratory--Variation that occurs in replicate tests or analyses conducted at different times over a longer period of time or under different conditions which vary with longer periods of time at a single laboratory.

3. Laboratory-to-Laboratory (or Between Laboratories)--Variation that occurs when different laboratories conduct the same tests or analyses.

The three sources of variation occur in each of the four main processes mentioned. For example, although sets of tests or analyses may exhibit good reproducibility when conducted within a short period, they are still subject to long-term within-laboratory variation (in addition to short-term withinlaboratory variation). Also, PNL is only one laboratory; results would vary from one laboratory to another, with the results of all laboratories varying around the true results. Hence, PNL's results, or the results of any single laboratory, are subject to laboratory-to-laboratory variation.

In the discussion that follows, variation in the "glass fabrication" process is not discussed. Because these tests involved the "as-analyzed" composition of only a single glass composition and its uncertainty, the 
fabrication variation about the "as-designed" glass was not a concern. Thus, only the glass analysis, leach testing (including sample preparation), and leachate analysis processes need be discussed.

Although the data collected during these tests are we11 documented and extensive, not all the data needed to estimate every component of uncertainty affecting the leach testing and analysis processes shown in Table 4.1 were generated. Oniy the short-term sources of variation are assessed because some of the data needed to estimate 1ong-term or 1aboratory-to-1aboratory variations are not available.

The ultimate interest is in quantifying the uncertainty in blankcorrected individual normalized concentration values $\left(N C_{i j k}\right)$ and averages of these values from replicate leach tests $\left(\overline{\mathrm{NC}}_{\mathbf{i} . \mathrm{k}}\right)$. This is accomplished through statistical error propagation techniques, which are discussed in detail and illustrated in Appendix B. To summarize the concept, error propagation provides for combining the uncertainties in the $C_{i j k}, \bar{B}_{i}$, and $\bar{f}_{i}$. portions of the definition of $\mathrm{NC}_{i j k}$ [given earlier in Equation (3-1)] to yield the uncertainty in $\mathrm{NC}_{i j k}$. Relative to Table 4.1 , variations in the glass analysis process show up and are assessed as variations in the glass

Table 4.1. Illustration of the Nine Variance Components that must be Estimated to make Defensible Qualification Statements

$\begin{gathered}\text { Short-term } \\ \text { Within-Laboratory Within-Laboratory }\end{gathered}$ Laboratory-to-
Laboratory

G1ass Fabrication and Analysis Process

Leach Testing Process

Leachate Anatysis Process

(a) Indicated variance components addressed by the current testing. (b) Variance components not addressed by the current testing. 
weight fractions $\bar{f}_{i}$, while variations in the leach testing and leachate analysis processes show up as variations in $C_{i j k}$ and $\bar{B}_{i}$. values. $B l$ ank corrections for leach tests performed with deionized water as the leachant typically add little to the uncertainty of normalized concentration values.

The error propagation formulas presented in Appendix B were applied to assess the short-term uncertainty of individual and averaged normalized concentration values. The resulting standard deviations of individual normalized concentration values were used to construct the error bars shown in Figures 3.5 and 3.6. The construction of these error bars is now discussed.

The normalized concentration values plotted in Figures 3.5 and 3.6 are the averages of results from triplicate specimens at each set of test conditions. There are several options for describing the uncertainty in the plotted values. One way is to define the error bars as $95 \%$ confidence limits on the mean normalized concentration values. Alternatively, the uncertainty of individual (rather than mean) normalized concentrations can be displayed by defining the error bars as $95 \% / 95 \%$ tolerance intervals. In both of these options, however, the uncertainties are relative to only one $(S A / V) \cdot t$ value at a time. If interest is in the relationship of normalized concentration to $(S A / V) \cdot t$, then $95 \%$ confidence bands or $95 \% / 95 \%$ tolerance bands on a fitted model could be displayed. These options would illustrate the uncertainty in the whole curve (considered as means or individual normalized concentrations) across all $(S A / V) \cdot t$ values instead of at just one $(S A / V) \cdot t$ value at a time. It was beyond the scope of work to develop confidence or tolerance bands for the curves in Figures 3.5 and 3.6, but doing so is recommended for future work in which the relationship between normalized concentration and $(S A / V) \cdot t$ is of interest.

It should be stressed that all four of the options discussed above would illustrate only short-term within-lab variations (uncertainty) in normalized concentrations, since that is the only variation information present in the data. Error bars for any of the ways of quantifying uncertainty would be considerably wider if long-term within-lab or lab-to-lab variations were included. 
The error bars in Figures 3.5 and 3.6 were obtained via the $95 \% / 95 \%$ to 1 erance interval approach. This means we have 95\% confidence that the interval for a given (SA/V) $t$ value contains $95 \%$ of the potential results from replicate tests (performed at the same time). The technique applied to construct the $95 \% / 95 \%$ tolerance intervals assumes that the distribution of potential normalized concentrations at a given $(S A / V) \cdot t$ value is normally distributed. Note that a $95 \% / 95 \%$ tolerance interval is a statement about the 95\% of the distribution of normalized concentration values for a specific $(S A / V) \cdot t$ value, and not a statement about the mean of the distribution (which is what a $95 \%$ confidence limit would provide).

The difference between tolerance and confidence intervals is shown by the example calculation in Table 4.2 for the Al example used in Appendix B. Note that the width of the tolerance interval is considerably larger than the width of the confidence interval.

Table 4.2 applies to the population defined by MCC-3 leach tests (as applied in this study), at $90^{\circ} \mathrm{C}$ (nominal), for $1.067 \mathrm{~g}$ of $\mathrm{HW}-39-1 \mathrm{glass}$ ground from PNL's Batch 8 and screened to particle diameter of $74 \mu \mathrm{m}$ to $149 \mathrm{\mu m}$, leached in $40 \mathrm{ml}$ of DIW, (for a nominal SA/V of $530 \mathrm{~m}^{-1}$ ) for 182 days and the resultant leachates analyzed by PNL'S ICP laboratory on a specific day in 1987. PNL'S ICP laboratory also provided the mass fraction oxides in the unleached glass. Each of these population restrictions implies a potential source of variability, since an attempt to rerun this set of conditions would experience many variations from the specifications. Even if the test could be rerun exactly, the day of analysis would be different, and the ICP results would reflect the aging and/or recalibration of the equipment and the use of older or fresh standards. These factors reinforce the position that the estimated variances only quantify short-term within-lab variations.

In terms of characterizing the leachability of HW-39-1 glass, this set of tests provides data to estimate less than $10 \%$ of the total variability. This assessment is optimistic; the MCC-1 round robin results (12) showed only $5 \%$ of the total variability resulting from the combined short- and long-term within-lab components. The interpretation of the tolerance limit-based error bars cannot go beyond the 41 statistical populations defined by the 
TABLE 4.2. Comparison of Tolerance and Confidence Intervals for the A1 Example Considered in Appendix B

\begin{tabular}{lcc} 
& $\begin{array}{c}95 \% / 95 \% \text { Tolerance } \\
\text { Interval }\end{array}$ & $\begin{array}{c}95 \% \text { Confidence } \\
\text { Interval }\end{array}$ \\
\cline { 2 - 2 } Mean & 260.65 & 260.65 \\
Standard Deviation & 8.4976 & 7.6248 \\
Tolerance or Confidence & & \\
Factor for $n=3$ & $9.916(\mathrm{a})$ & $4.303(\mathrm{~b})$ \\
Lower Limit & 176.39 & 227.84 \\
Upper Limit & 344.91 & 293.46 \\
Half Width & 168.52 & 32.81 \\
Half-Width (\% Relative Mean) & 32.2 & 12.6
\end{tabular}

(a) From Table A-6, Factors for Two-Sided Tolerance Limits for Normal Distribution, p. T-10 in NBS Handbook 91, 1963.

(b) From Appendix 5, Cumulative t-distribution, p. 528 in Statistics in Research by B. Ostle, Iowa Fate M. Press, 1963.

combination of test conditions used in this study. They do reflect the short-term, within-lab variability attributable to glass ICP analysis, leach testing (specimen makeup and testing), and leachate analysis processes for these populations.

\subsection{RECOMMENDATIONS FOR CHANGES TO THE LEACH TESTING METHODOLOGY}

The "true" composition of a glass being tested cannot be known. Nor can the true elemental release that results from leach testing of a glass be known. These quantities can only be estimated based on chemical analyses of the glass and leachate. Because of the uncertainties associated with these analyses and with the leach testing process, appropriate data must be collected to estimate the uncertainties.

In computing the uncertainty in estimates of glass composition, both short- and long-term within-laboratory variation, as well as between-lab variation, must be included. The situation is more complicated for elemental release data; short- and long-term within-laboratory variation and betweenlab variation for both the leach testing process and the leachate analys is 
process must be included. If the elemental release data are normalized to the corresponding estimated glass composition, then the glass analys is uncertainties must also be included.

In this section, changes to the test methodology are recommended for the collection of data required to estimate the various components of uncertainty affecting the leach testing and analysis processes. There are nine such components of uncertainty or "variance components" of interest, as illustrated in Table 4.1.

\subsubsection{Parallel Testing with an "Appropriate Certified Standard Glass"}

Glass analyses, leach tests, and leachate analyses are subject to longterm within-lab and 1ab-to-lab uncertainty even if tests are performed only at one time at one laboratory. Sti11, it is possible to adopt a testing and analysis methodology that removes these sources of variation from consideration. An appropriate certified standard glass can be analyzed for composition and leach tested in parallel with the other glass(es) being tested. The differences (or ratios) between the standard glass values for a given time and given laboratory as compared with the certified values can be used to "bias correct" the long-term within-1ab and 1ab-to-1ab effects of the results. Uncertainties resulting from the "bias correction" procedure must be taken into account, but they are typically much smaller than uncertainties resulting from long-term within-1ab and 1ab-to-1ab variations.

The phrase "appropriate certified standard glass" requires some explanation. "Appropriate" means that it must have a composition representative of the glass(es) being tested, otherwise the "bias correction" may not be appropriate. "Certified" means that the glass must have previously been tested to the extent that the composition, leach behavior, and standard deviations (short-term, long-term, and 1ab-to-1ab) are a11 we11-known. This testing could be accomplished via a large round robin, although it would have to be structured to estimate al1 the sources of variation of interest. A "standard" glass is one that is available in sufficient quantities to allow for routine, parallel testing with "unknown" glasses to allow for bias correction and uncertainty quantification. 
Appropriate certified standard glasses fully meeting this definition do not exist, so it isn't possible to fully implement this methodology. Still, compositional analyses and leach testing of some standard glasses along with other glasses being tested is recommended. In the meantime, it is recommended that round robin testing be conducted to develop appropriate certified standard glasses. Long-term within-lab and lab-to-lab variations contribute almost all of the total uncertainty in normalized concentration values. To demonstrate compliance with the eventual WAPS specifications on elemental and radionuclides releases, long-term within-lab and lab-to-lab uncertainties will either have to be included in uncertainty estimates (which could make it considerably more difficult to demonstrate compliance) or compensated for via a methodology similar to that discussed above.

\subsubsection{Replication and Randomization}

Replication and randomization are the techniques used to obtain the data necessary to determine the uncertainty in estimated glass compositions or elemental release values. Replication of tests and analyses is needed to provide data for estimating the uncertainties resulting from the sources of variation. Randomizing the order of tests and analyses provides for correct estimation of the uncertainties by allowing the full range of uncontrolled variability to have its effect on the estimates.

In considering replication, "complete" and "partial" replication are differentiated. For example, complete replication relative to the leaching process would involve leaching each test condition a specified number of times. With partial replication, only some of the leach tests would be repeated. In the work discussed in this report, complete replication was used in the leach testing process (each glass was tested in triplicate and each of the three leachates analyzed once). Similarly, complete replication was used in performing the glass composition analysis. Each of the three batches of HW-39-1 glass was analyzed for composition three times. Because the glass analysis, leach testing, and leachate analysis replications were performed at the same time by a single laboratory, the replication methodology is inadequate for estimating all of the variance components in Table 4.1. 
Complete replication not only provides data for computing the uncertainty of the process being replicated, it also provides sufficient data to reduce the uncertainty. Specificaliy, the uncertainty is effectively reduced by averaging the replicate results and using the standard error instead of the standard deviation as the measure of uncertainty. (The standard error is the standard deviation divided by the square root of the number of replicates.) The problem with complete replication is that it can be very expensive. The alternative is partial replication. Although partial replication provides data for estimating the uncertainty, it doesn't provide sufficient data for across-the-board reduction in the uncertainty via averaging. Future testing should evaluated on a case-by-case basis by balancing the cost of replication verses the required reduction in the uncertainty.

Completely randomizing the order of tests is not practical when longerterm leach tests are being conducted. A11 year-long and 6-month tests wil1 start at the beginning of the testing period to ensure their completion. When possible, shorter duration tests shouid be conducted in a random order over the total testing period if possible. Furthermore, all tests of a given duration will start at the same time (except for replicates planned to start at other times). When samples are sent out for analysis, the order in which the samples are analyzed should aiso be randomized.

\subsubsection{Glass Analysis--Short- and Long-Term Within-Laboratory Variation}

To establish both short- and long-term within-laboratory variation in the glass analysis process, each glass used in the leach testing should be analyzed at four different times. Such analysis provides data for a joint estimate of the short- and long-term within-laboratory variance components. Also, at the first two times, each glass should be analyzed twice, which provides data for estimating the short-term within-laboratory variation. These two aspects of the scheme allow for separate estimation of the shortand long-term within-laboratory variance components. If a large number of glasses are to be tested, multiple analyses need be conducted on onty a few of the glasses instead of on all of the glasses to establish and estimate of the variance components. 
The four times that each glass is analyzed should be far enough apart to include all long-term sources of variation. The duplicate analyses performed at each of the first two (of the four) times should be performed far enough apart (i.e., not immediately after one another) so that the short-term sources of variation are included. Each time glass samples are analyzed for composition, the analyses should be performed in random order.

A standard glass such as an $\operatorname{MCC}(a)$ approved reference material (such as ARM-1) or an MCC approved testing material (ATM-10) should be analyzed in duplicate with each set of glasses analyzed over the life of the tests. For a standard glass to be of the greatest use, it should be as representative of the tested glass composition as possible and should have well-known estimates of its composition and the corresponding uncertainty (including short-, longterm, and lab-to-lab sources of uncertainty). The standard glass data may be useful for estimating and correcting for any biases in the ICP analyses of the glass.

\subsubsection{Glass Analysis--Lab-to-Lab Variation}

To estimate lab-to-lab variation in analyses of glass composition, data from more than one laboratory are required. One option is to send two or three of the glasses to be leached to several other laboratories for analysis. It is important to involve several laboratories (at least 5 or 6). Another option, which may not be entirely satisfactory, is to use the results of other lab-to-lab round robin studies to estimate the lab-to-lab variability in glass analyses.

\subsubsection{Leach Testing and Leachate Analysis--Short- and Long-Term Within-Laboratory Variation}

Current practice is to perform the leach tests in triplicate and analyze each leachate only once. Because the triplicate leachates have been analyzed sequentially (with the ICP) to this point, only very short-term instrument variation is included. In future testing, it will be necessary to implement proper randomization in the analysis of the leachates from the replicate

(a) Materials Characterization Center. Operated by Pacific Northwest Laboratory, Richland, Washington. 
tests. In addition, the analyses should be spread over a long-enough period of time to pick up all short-term variations.

To allow for the estimation of both short-and long-term variance components for leach testing and leachate analysis, the following general recommendations are made:

- Each individual leach test should continue to be performed in triplicate (with one analysis per leachate) to provide data for a short-term within-laboratory variance estimate that combines the variance components of leach testing and leachate analysis. Because triplicate testing involves complete replication, the impact of short-term uncertainty is reduced by averaging the replicate results.

- Sets of leach conditions (the number would depend on the size of the test matrix and the required limits on uncertainties) should be tested (in triplicate) at different times to provide data for a long-term within-laboratory variance estimate that combines the variance components of leach testing and leachate analysis. The times should be far enough apart to include all long-term sources of variation affecting the two processes. The triplicate tests at each time provide data for a short-term variance estimate that combines the variance components of leach testing and leachate analysis. In addition, performing triplicate tests at each time allows the short and long-term variation to be estimated separately.

- Leachates from each of the replicated tests recommended above should be analyzed at different times to provide data for estimating the long-term within-1aboratory variation of leachate analysis only. At the first two times, duplicate analyses should be performed (on the three leachates from each test) to provide data for estimating the short-term within-7aboratory variation of leachate analysis only. Without duplicate analyses, the variation estimate is a combination of the leach testing and leachate ana7ysis variance components. Although a combined estimate is acceptable for evaluation of total uncertainty, separate estimates of short- and long-term variation for the leach testing and leachate analysis processes may be very enlightening. An important aspect of this part of the replication scheme is that enough leachate from a single test (for each of the three glasses) needs to be available to allow for the analyses (two at the first time, two at the second time, and one each at the third and fourth times for this example). The leachate must be from a common leach test, not from replicate tests performed on each glass. This will be possible for the MCC-1 and $M C C-3$ at $S A / V$ values of 530 and $2000 \mathrm{~m}^{-1}$. There is also a concern that leachate samples may not be stable when held for longer periods of time. A "standard" leachate could also be used to determine leachant analysis variation if the standard leachate is 
representative of the leachates. Analysis of a standard leachate in the year prior to testing and during the testing period would establish the stability of the leachates and provide additional data for variance estimation.

Proper randomization should be used at all stages of leach testing and leachate analysis. For example, the set-up of the leach tests should be completely random, including the three replicates for each glass (i.e., the triplicates should not be setup one after the other).

\subsubsection{Leach Testing and Leachate Analysis--Lab-to-Lab Variation}

Estimating 1ab-to-1ab variation for the leach testing and leachate analys is processes is beyond the current scope of work. However, it is important from the standpoint of final qualification statements, so some options for future testing are given below.

One option is to send one or two glasses to several laboratories (five or six at least) for leach testing and subsequent leachate analyses. This option would provide some data for a combined estimate of the 1ab-to-1ab leach testing and leachate analysis variance components. If separate estimates of these two variance components are desired, a standard leachate (representative of the leachates for the compositional region of interest) should be submitted to each of the laboratories for analysis. This would provide for a separate estimate of lab-to-lab leachate analysis variation, which could then be applied to the combined estimate to obtain a separate leach testing variance component estimate.

An option that may not be entirely satisfactory, but would be of immediate help, is to use the results of other lab-to-lab variation studies. The results of documented round robin testing $(12,13)$ might be usefur in estimating the 1ab-to-1ab variability in the $M C C-1$ test results. The results from the Product Consistency Test round-robin being conducted by the Materials Characterization Center for SRL in FY 1988 might provide data useful for estimating the lab-to-lab uncertainty associated with the 7-day MCC-3 tests and possibly the 28-day tests. 


\subsection{COMPARISON WITH MODIFIED MCC-3 TESTING}

A modified MCC-3 testing procedure has been used previously on a limited basis to evaluate the leaching characteristics over a wide range of leachant saturation conditions for a number of nuclear waste glasses, including HW-39-1.(14) Using this technique, multiple leachate samples are extracted from a single dissolution test, the extracted solution is replaced. This technique allows releases to be approximated at the lower (SA/V) $t$ conditions with fewer test samples and reduced cost and time requirements. The modified MCC -3 technique has been used in other programs with good results. $(15,16)$ By comparing the results from the modified MCC-3 testing with the leach testing reported here, it was shown that the modified MCC-3 technique produces comparable results to those of the standard MCC-1 and MCC-3 techniques.

The modified MCC-3 test is a partial leachant replacement MCC-3 leach test; one leach vessel is used for each SA/V range to be tested, and multiple samples are taken over a period of time. The small amount of leachate removed is replaced with fresh leachant at the time of each sampling. For example, testing could be conducted at SA/V ratios of 50,2000 and $10000 \mathrm{~m}^{-1}$. A modified MCC-3 dissolution test would use $1 \mathrm{~g}$ of $-100+200$ mesh glass powder added to $400 \mathrm{ml}$ of deionized water and placed in a $90^{\circ} \mathrm{C}$ oven. This would provide an $S A / V$ ratio of $50 \mathrm{~m}^{-1}$. At specified time intervals, a 5-ml sample of leachate would be removed and replaced by $5 \mathrm{ml}$ of fresh deionized water. Using this technique, multiple leachate samples can be extracted from a single dissolution test. For $S A / V$ ratios of $2000 \mathrm{~m}^{-1}, 7 \mathrm{~g}$ of $\mathrm{glass}$ would be added to $70 \mathrm{ml}$ of leachant. For a SA/V ratio of $10000 \mathrm{~m}^{-1}, 10 \mathrm{~g}$ of glass would be added to $20 \mathrm{ml}$ of leachant. A single glass powder size, such as $-100+200$ mesh, should be used for all tests rather than introducing any uncertainties resulting from particle size distribution differences at different mesh sizes.

Because the modified MCC-3 technique is essentially a very slow flowing leach test and not a static type test, data from the modified MCC-3 technique can only be used to assess the $(S A / V) \cdot t$ scaling at lower values of the flow rate to forward rate of dissolution ratio. At the lower $S A / V \cdot t$ values, the volume replacement is small compared to the total leachant volume, and the 
dissolution rate is high enough so that the elements (primarily silicon) removed in the sample have a negligible effect on the dissolution rate. To obtain higher values for $(S A / V) \cdot t$ scaling, standard MCC-3 techniques would be used.

Glass dissolution data approximately follow the (SA/V) - t scaling law in static tests (below SA $/ V$ ratio of 20,000 ), indicating that the rate for dissolution of the glass can be appropriately described as a function of concentration of species in solution. This relationship constitutes a dissolution rate law. (17) The basic objective of modeling the dissolution of an HWVP glass is to determine this rate law as a function of concentration. This is facilitated by a test method that allows the concentrations in solution to be varied over the range of interest while simultaneously allowing the rate of dissolution to be monitored. The partial replacement MCC-3 test appears to be well-suited for this purpose. In this test, the concentration should eventually come to a steady-state value that depends on the ratio of the volume replaced per sampling to the surface area of the glass, the ratio of sample to total leachate volume, and sampling frequency. The time required to reach the steady-state will depend on the glass SA/V ratio. When steadystate is achieved, the dissolution rate is found from the replacement-to-area ratio and the steady-state concentrations. The form of the dissolution rate law can also be tested by comparing the resulting predicted transient behavior of the concentration data, since the concentration is known during its approach to its steady-state by virtue of examination of the solution that is removed and replaced.

Previously (1986) conducted tests characterizing the dissolution behavior of the HWVP HW-39-1 reference glass were performed using a mixture of $M C C-1, M C C-3$, and modified MCC-3 techniques at $S A / V$ values of $50,2,000$ and $10,000 \mathrm{~m}^{-1}$ (see example on previous page). In Figures 5.1 and 5.2 , the $B$ and Si results from the modified $M C C-3$ tests conducted previously are plotted with the $B$ and $S i$ results (Figures 3.5 and 3.6 ) from the $M C C-1$ and $M C C-3$ leaching tests reported here.

The data from the modified MCC-3 testing agree very well (in most cases to within \pm 2 standard deviations) with the data produced using the standard 


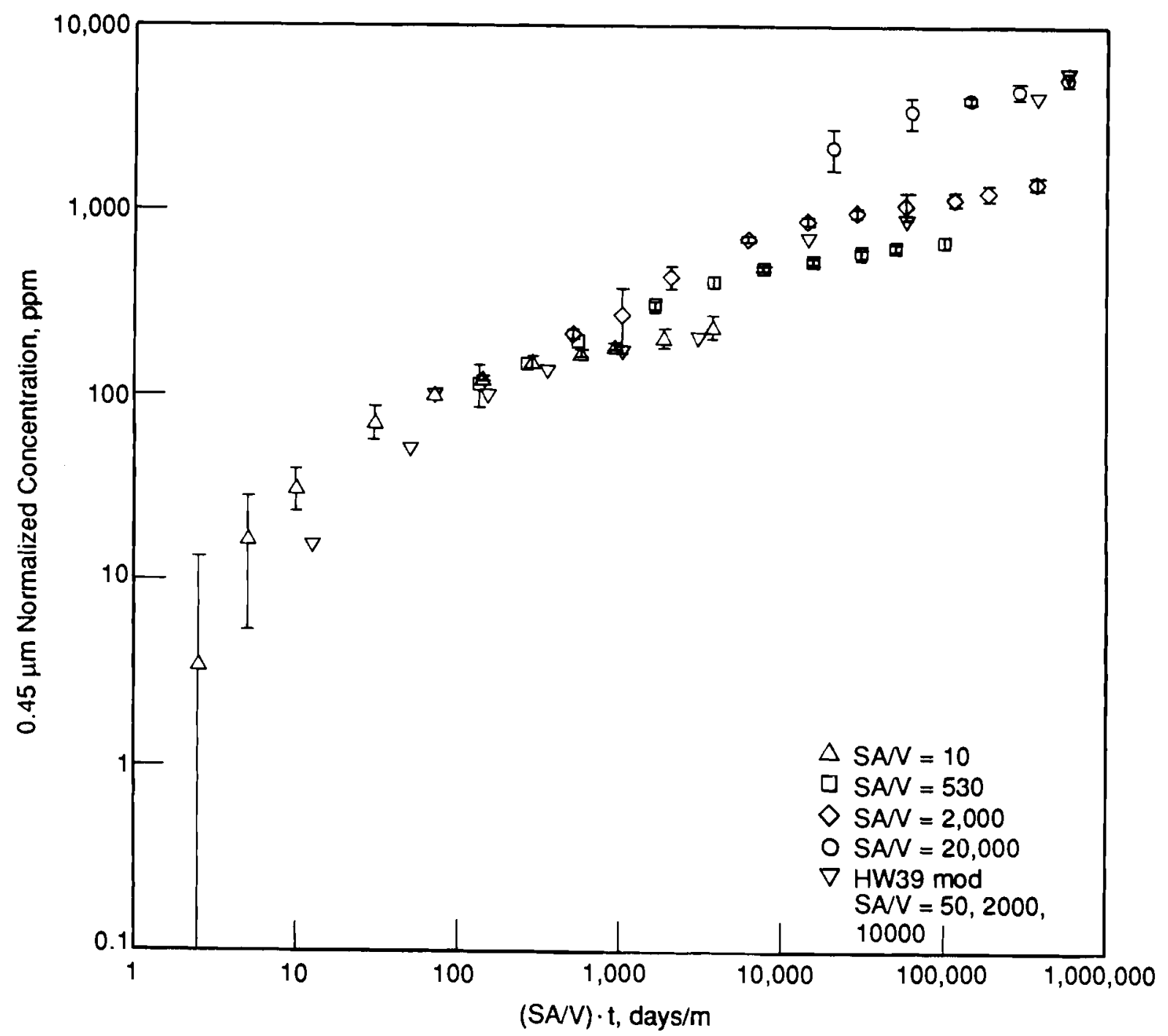

FIGURE 5.1. Comparison of Normalized Boron Concentrations from HW-39-1 Product Leach Test Strategy and a Modified MCC -3 Methodology

MCC -1 and MCC -3 leach tests. The modified MCC -3 data appear to be slightly lower at the low $(S A / V) \cdot t$ values where the MCC-1 procedure was used. By plotting the unit slope at the lower values of $(S A / V) \cdot t$ values for the modified MCC-3 data back to the $y$-intercept, a forward dissolution rate of approximately $1.2 \mathrm{~g} /\left(\mathrm{m}^{2}\right.$-days) can be established using the modified MCC -3 technique and compared with the rates obtained from standard MCC-1 and MCC -3 testing. This rate compares well with the $1.5 \mathrm{~g} /\left(\mathrm{m}^{2}\right.$.days $\rangle$ rate established using the MCC-1 and MCC-3 techniques in the waste form qualification leach 


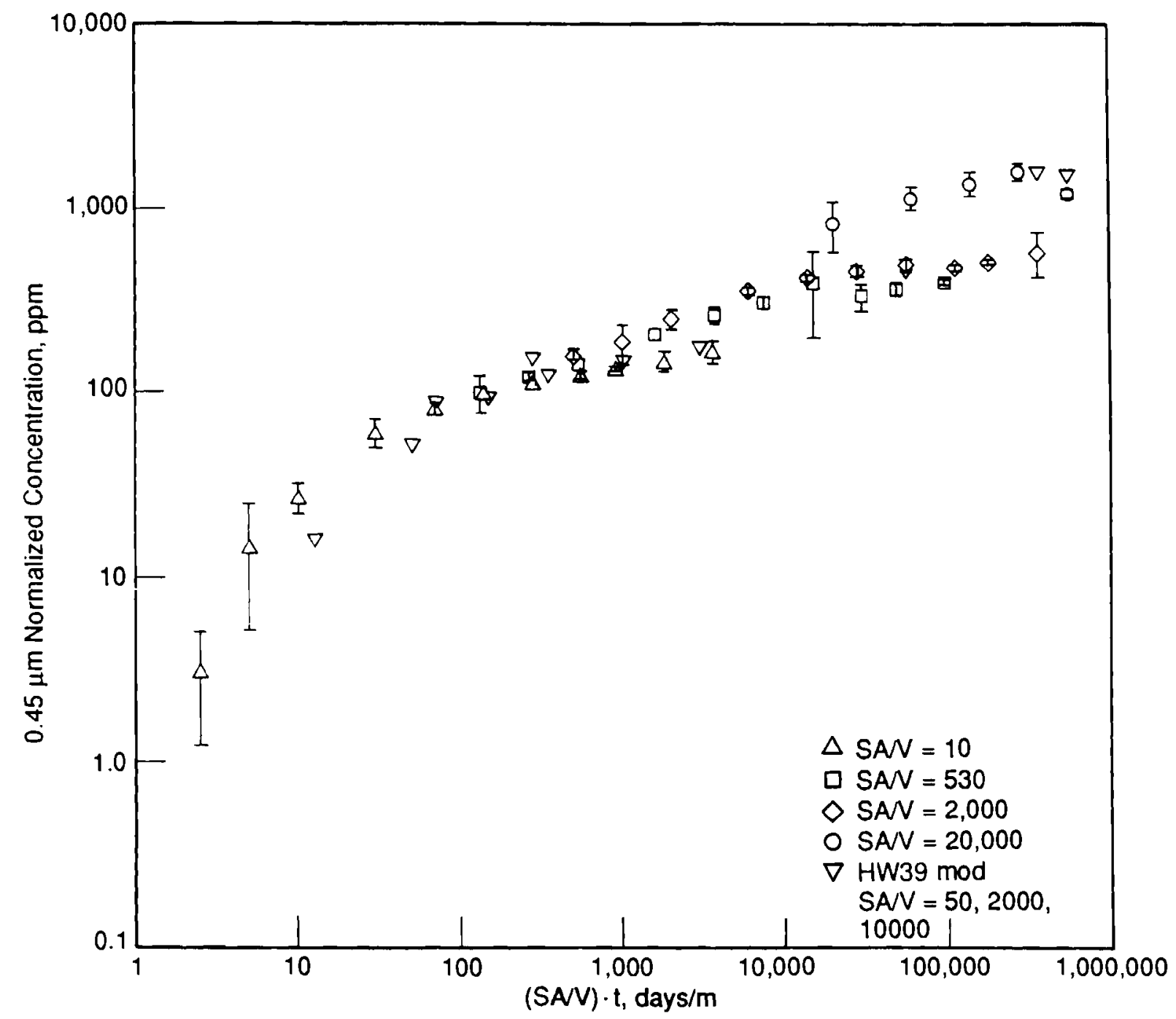

FIGURE 5.2. Comparison of Normalized Silicon Concentrations from HW-39-1 Product Leach Test Strategy and a Modified MCC-3 Methodology

testing. In other studies using the modified MCC -3 technique, the approximate forward reaction (dissolution) rate was determined to be $0.8 \mathrm{~g} /\left(\mathrm{m}^{2} \cdot\right.$ days) for DWPF SRL-165 glass, and $1.6 \mathrm{~g} /$ (m2.days) for PNL 76-68. (16)

It is recommended that the modified MCC-3 procedure be used in future testing to determine the dissolution characteristics over a wide range of solution saturation conditions because of the lower cost and time required to produce analogous data over a wide range of saturation conditions. MCC-1 and MCC-3 tests of 7- and 28-day duration should also be conducted to corroborate 
test results. The 7- and 28-day MCC-1 and MCC-3 tests conditions are the most commonly reported and likely to be chosen for comparison. The recommended modifications to the leach testing methodology presented in Section 4.5 should also be applied to the testing conducted with the modified MCC -3 procedure so that variance components can be estimated. 
.

*

. 


\subsection{CONCLUSIONS}

The following conclusions are made based on the analysis of the leach testing of the HWVP reference glass (HW-39-1).

- Elemental concentrations in the leachates increased with increasing $S A / V$ ratios at comparable $(S A / V) \cdot t$ values. The $(S A / V) \cdot t$ scaling method provides an estimate of glass dissolution at concentration conditions that will occur as a result of the glass dissolution. However, the concentration conditions at given $(S A / V) \cdot t$ values obtained using higher $S A / V$ values do not correlate well with those obtained using the low $S A / V$ values. Thus, the $(S A / V) \cdot t$ scaling technique appears to be adequate to provide a general indication of the dissolution characteristics of HW-39-1 glass in solution concentrations produced with $S A / V$ values up to $2000 \mathrm{~m}^{-1}$ but is inadequate when the $S A / V$ value is increased to $20,000 \mathrm{~m}^{-1}$.

- The same trend observed for elemental solution concentrations was also observed for $\mathrm{pH}$. Tests at higher SA/V values produced higher leachate $\mathrm{pH}^{\prime} \mathrm{s}$ at comparable $(\mathrm{SA} / \mathrm{V}) \cdot \mathrm{t}$ values. This $\mathrm{pH}$ deviation may help explain the higher normalized concentrations in the leachate at similar $(S A / V) \cdot t$ values. The cause of the $\mathrm{pH}$ deviation is not yet understood.

- Based on the standard MCC-1 and MCC-3 leach testing conducted, the initial rate of glass dissolution of the HW-39-1 glass was estimated to be $1.5 \mathrm{~g} /\left(\mathrm{m}^{2} \mathrm{~d}\right)$ and the steady-state dissolution rate was estimated to be less than $0.005 \mathrm{~g} /\left(\mathrm{m}^{2} \mathrm{~d}\right)$ based on $B$ release. At the test conditions specified in the draft WAS for qualification (MCC-1, 28-day, $90^{\circ} \mathrm{C}, 10 \mathrm{~m}^{-1}$ ), the HW-39-1 glass has a $B$ release of $0.53 \mathrm{~g} / \mathrm{m}^{2} \mathrm{~d}$, which is well below the specified $1 \mathrm{~g} / \mathrm{m}^{2} \mathrm{~d} 1$ imit.

- Although the current leach testing methodology provides a means for calculating some of the sources of variability in the test results, other sources of variance could not be estimated from the available test results. However, with some additions and modifications to the testing methodology (outlined in the recommendations in Section 7.0 ), the data required to estimate these other variance components can be generated in subsequent testing so that sufficiently defendable qualification statements can be made.

- Uncertainty in elemental analysis of the glass appears to be a significant contributor to the short-term within-1aboratory uncertainties associated with normalized concentrations. For normalized concentrations of Al from MCC-3 testing of HW-39-1 with an SA/V value of $530 \mathrm{~m}^{-1}, 70 \%$ of the uncertainty was due to glass analyses, while nearly $30 \%$ was due to uncertainties from the leach testing and leachate analysis processes. The percentage contributions may 
vary considerably from element to element. Uncertainty related to blank corrections is a minor contributor for tests conducted using DIW as the leachant.

- Data generated by the modified MCC-3 leach testing technique are very similar to the data generated by the test matrix used in this leaching study at given (SA/V) - $t$ values. The modified MCC-3 technique provides the data faster (at the $(S A / V) \cdot t$ values), with less sample preparation, and at lower cost than the methodology used in this report.

- The HWVP reference glass (HW-39-1) dissolves congruently in deionized water at early times. It is likely that congruent dissolution also occurs at later times, but this claim cannot be substantiated from the solution concentrations of the glass constituents because some of the constituents precipitate from solution. 


\subsection{RECOMMENDATIONS}

The following recommendations for WFQ laboratory work and development are made based on the results and analyses reported herein.

- For $(S A / V) \cdot t$ scaling to be used to evaluate glass dissolution behavior, the SA/V rafio used in MCC-3 testing should not exceed approximately $2000 \mathrm{~m}^{-1}$ in deionized water. Additional testing should be conducted to determine the reasons for the increase in elemental release with increasing $S A / V$ ratios at comparable $(S A / V) \cdot t$ values.

- A11 tests should be conducted in a random order over the total testing period if possible. When glass and leachate samples are sent out for analysis, the order in which the samples are analyzed should also be random. A random order of testing and analysis permits more accurate estimates of the uncertainties.

- A standard glass should be analyzed in duplicate with each set of glass analyses over the life of the tests. Glasses such as an MCC approved reference material (ARM) or an MCC approved testing material (ATM) could be used for this purpose. For a standard glass to be of the greatest use, it should be as representative of the tested glass composition as possible and should have we11-known estimates of its composition and of short-term, long-term, and labto-1ab sources of uncertainty. Currently available standard glasses should be evaluated to determine if a more appropriate standard glass should be developed. Al so, it may be necessary to conduct round robin leach testing and analysis of the standard glass to obtain "certified" values of its composition and leach rates and their corresponding uncertainties.

- Elemental composition data from more than one laboratory should be obtained to estimate 1ab-to-1ab variation in analyses of glasses or leachates.

- If a number of glass compositions are to be used in the leaching test, several of the glasses (or each glass if there are only a few) should be analyzed (for elemental composition) at four different times at least two weeks apart, with duplicate analyses at the first two times. This provides for estimating both shortand long-term within-laboratory variation in the glass analys is process.

- In addition to triplicate testing of individual leach tests to provide short-term within-lab variance estimates, sets of leach conditions should be tested (in triplicate) at different times to provide data for a long-term within-lab variance estimate that combines the variance components of leach testing and leachate analysis. 
- Leachates from each of the replicated tests recommended above should be analyzed at four different times to provide data for estimating long-term within-lab variation of leachate analys is only. Duplicate analyses should be performed on a statistically determined number of leachates from each test (depending on the total number of tests and the length of the testing period) to provide data for estimating short-term within-lab variation of leachate analysis only. Leachate stability also needs to be verified.

- The modified MCC-3 (partial leachant replacement) procedure should be used in future testing to determine the dissolution characteristics over a wide range of $(S A / V) \cdot t$ values. This modified MCC -3 procedure provides data faster (at lower $(S A / V) \cdot t$ values) and requires less sample preparation, resulting in lower cost to produce analogous data (compared with standard MCC -1 and MCC-3 testing). MCC-1 and MCC-3 tests of 7- and 28-day duration should also be conducted to provide confirmation of test results. The recommended modifications to the leach testing methodology should also be applied to the testing conducted with the modified MCC-e procedure so that variance components can be estimated. 


\subsection{REFERENCES}

1. U.S. Department of Energy. 1986. Waste Acceptance Preliminary Specifications for the Defense Waste Processing Facility High Level Waste Form. ORG/B-8, Draft for Concurrence, Apri1 1986, U.S. Department of Energy, Washington, D.C.

2. U.S. Department of Energy. 1986. Waste Acceptance Preliminary Specifications for the West Valley Demonstration Project High Level Waste Form. Draft for Concurrence, Apri1 1986, U.S. Department of Energy, Washington, D.C.

3. Code of Federal Regulations. 1983. "Disposal of High-Level Radioactive Waste in Geological Repositories: Technical Criteria." 10 CFR60. Nuclear Regulatory Commission, Washington, D. C.

4. Nelson, J. L. 1988. Hanford Waste Vitrification Plant Waste Form Qualification Program P1 an - FY 1988 Update, Rev. 1., WHC-EP-0045. Westinghouse Hanford Company, Richland, Washington.

5. Lokken, R. O., and D. M. Strachan. 1984. "Long-Term Leaching of Two Simulated Waste Glasses." In Nuclear Waste Management. Advances in Ceramics. Vol. 8, eds. G. C. Wicks and W. A. Ross, pp. 39-48, American Ceramic Society, Columbus, Ohio.

6. Materials Characterization Center. 1983. MCC-1P Static Leach Test Method. Pacific Northwest Laboratory, Rich1and, Washington.

7. Materials Characterization Center. 1984. MCC-3 Agitated Powder Leach Test Method. Pacific Northwest Laboratory, Richland, Washington.

8. Fullam, H. T. 1981. Solubility Effects in Waste Glass/DemineralizedWater Systems. PNL-3614, Pacific Northwest Laboratory, Richland, Washington.

9. McVay, G. L., D. J. Bradley, and J. F. Kircher. 1981. Elemental Release from Glass and Spent Fuel. ONWI-275, Office of Nuclear Waste Isolation, Pacific Northwest Laboratory, Richland, Washington.

10. Grambow, B. E. 1985. "A General Rate Equation for Nuclear Waste Glass Corrosion." In Scientific Basis for Nuclear Waste Management VIII, C. M. Jantzen, J. A. Stone, and R. C. Ewing, editors, Materials Research Society, Pittsburgh, Pennsylvania.

11. Coles, D. B., and M. J. Apted. 1984. "The Behavior of ${ }^{99} \mathrm{Tc}$ in DopedGlass/Basalt Hydrothermal Interaction Tests." Scientific Basis for Nuclear Waste Management VII. ed. G. L. McVay, North-Holland, New York. 
12. Konnecke, R., and J. Kirsch. 1985. EC Static High-Temperature Leach Test--Summary Report of a European Community Interlaboratory Round Robin. EUR 9772 EN Commission of the European Communities.

13. Johnston, J. W., and J. L. Danie1. 1982. Summary Report for the Interlaboratory Round Robin on the MCC-1 Static Leach Test Method. PNL-4249. Pacific Northwest Laboratory, Richland, Washington.

14. Bunnell, L. R., G. D. Maup in, and K. H. Oma. "High-Temperature Glasses for Nuclear Waste Isolation." In Nuclear Waste Management II. Advances in Ceramics. Vol. 20, eds., D. E. Clark, W. B. White, and A. J. Machiels, pp. 167-173, American Ceramic Society, Columbus, Ohio.

15. McGrail, B. P., et al. 1988. "Obsidian Hydration Dating - Field, Laboratory, and Modeling Results," Mat. Res. Soc. Symp. Proc., Vol. 125. Materials Research Society, Pittsburgh, Pennsylvania.

16. Carter, G. D., S. Koegler, and S. 0. Bates. 1988. ORNL In-Situ Vitrification Pilot-Scale Test Process and Product Evaluation. PNL-6530, Pacific Northwest Laboratory, Richland, Washington.

17. Kuhn, W. L., and R. D. Peters. 1983. "Leach Models for a Commercial Nuclear Waste Glass." In Materials Research Society Proceeding, Vol. 15, pp. 167, Materials Research Society, Pittsburgh, Pennsylvania. 


\section{APPENDIX A}

\section{TABLES OF ELEMENTAL AND NORMALIZED CONCENTRATION RESULTS}


,

. 
APPENDIX A

\section{TABLES OF ELEMENTAL AND MORMALIZED CONCENTRATION RESULTS}

This appendix contains a summary of the as-analyzed and normalized concentrations from leachate analysis obtained from the leach testing of HWVP HW-39-1 glass reported within the report. These data are reported in eight tables (A.1 through A.8). For all concentration tables, analysis was conducted by inductively coupled plasma atomic emission spectroscopy (ICP); elemental results are given in $\mathrm{ppm}$; normalized results are given in $\mathrm{g} / \mathrm{m}^{2}$. The concentrations reported are have been "blank corrected" by subtracting the elemental concentrations found in the blank from the elemental concentrations found in the leachate at the same $S A / V$ ratio and time conditions. Normalized results are calculated from blank-corrected data. Tables A.1 to A.4 present the results from each leach test conducted; Tables A.5 to A.8 present the average values for each set of triplicate tests for a given $S A / V$ ratio and time condition.

Normalized results from minor elements in the glass should not be considered as a true reflection of the release behavior of the glass. Normalized results for the elements found in very low levels (near the detection level in the glass) vary radically and in some cases are negative. The variance occurs because the blank-corrected concentration (which is usually below detection limits) is divided by the small values of elemental weight fraction in calculating the normalized values (Section 3.0). Analytical "noise" in the analysis of the blank or leachate resulted in a reported value that is the result of "noise" rather than a "true" value. Because of blank correction, some concentrations (not normalized) were also negative. Since these results were used in the determination of short-term analytical uncertainty, they are presented for completeness.

It should be noted that any data with a SA/V ratio of $10 \mathrm{~m}^{-1}$ is MCC-1 data and has not been filtered. These data have been placed in the tables of both $18 \AA$ and $0.45 \mu \mathrm{m}$ filtered data for convenience, but should not be confused with the MCC -3 data. 
The following is a listing of the Tables presented:

- A.1 Elemental concentrations (ppm) of $18 \AA$ filtered data for all leach tests.

- A.2 Elemental concentrations (ppm) of $0.45 \mu \mathrm{m}$ filtered data for all leach tests.

- A.3 Normalized concentrations $\left(\mathrm{g} / \mathrm{m}^{2}\right)$ of $18 \AA$ filtered data for all leach tests.

- A.4 Normalized concentrations $\left(\mathrm{g} / \mathrm{m}^{2}\right)$ of $0.45 \mu \mathrm{m}$ filtered data for all leach tests.

- A.5 Average elemental concentrations (ppm) of $18 \AA$ filtered data for each test condition.

- A.6 Average elemental concentrations (ppm) of $0.45 \mu \mathrm{m}$ filtered data for each test condition.

- A.7 Average normalized concentrations $\left(\mathrm{g} / \mathrm{m}^{2}\right)$ of $18 \AA$ filtered data for each test condition.

- A.8 Average normalized concentrations $\left(\mathrm{g} / \mathrm{m}^{2}\right)$ of $0.45 \mu \mathrm{m}$ filtered data for each test condition.

Tables A.1 through A.4 are each 21 pages long. Tables A.5 through A.8 are each 7 pages long. 
ELEMENTAL CONC. (ppm)FROM 18A FILTERED LEACHATE (ALL TESTS)

\begin{tabular}{|c|c|c|c|c|c|c|c|}
\hline $\mathrm{SA} / \mathrm{V}$ & Duration & & & & & & \\
\hline$(m-1)$ & (days) & $\mathrm{pH}$ final & $\mathrm{pH}$ in & AL & AS & B & BA \\
\hline 10 & 0.25 & 7.48 & 5.58 & 0.07 & 0.00 & 0.09 & 0.00 \\
\hline 10 & 0.25 & 7.34 & 5.58 & 0.09 & 0.00 & 0.11 & 0.00 \\
\hline 10 & 0.25 & 7.27 & 5.58 & 0.08 & 0.00 & 0.11 & 0.00 \\
\hline 10 & 0.5 & 8.97 & 5.88 & 0.27 & 0.00 & 0.39 & 0.00 \\
\hline 10 & 0.5 & 9.07 & 5.88 & 0.36 & 0.00 & 0.51 & 0.01 \\
\hline 10 & 0.5 & 9.03 & 5.88 & 0.39 & 0.00 & 0.55 & 0.01 \\
\hline 10 & 1 & 9.52 & 5.67 & 0.66 & -0.01 & 0.92 & 0.01 \\
\hline 10 & 1 & 9.53 & 5.67 & 0.67 & -0.01 & 0.94 & 0.01 \\
\hline 10 & 1 & 9.49 & 5.67 & 0.60 & -0.01 & 0.86 & 0.01 \\
\hline 10 & 3 & 9.68 & 5.37 & 1.41 & -0.01 & 2.17 & 0.01 \\
\hline 10 & 3 & 9.67 & 5.37 & 1.36 & 0.00 & 2.01 & 0.01 \\
\hline 10 & 3 & 9.65 & 5.37 & 1.34 & 0.00 & 1.98 & 0.01 \\
\hline 10 & 7 & 9.55 & 5.74 & 1.67 & 0.01 & 2.89 & 0.01 \\
\hline 10 & 7 & 9.51 & 5.74 & 1.68 & 0.01 & 2.94 & 0.01 \\
\hline 10 & 7 & 9.51 & 5.74 & 1.63 & 0.01 & 2.83 & 0.01 \\
\hline 10 & 14 & 9.72 & 5.74 & 1.85 & -0.01 & 3.52 & 0.01 \\
\hline 10 & 14 & 9.71 & 5.74 & 1.86 & -0.01 & 3.54 & 0.01 \\
\hline 10 & 14 & 9.71 & 5.74 & 1.86 & -0.01 & 3.54 & 0.01 \\
\hline 10 & 28 & 9.34 & 6.01 & 2.04 & 0.01 & 4.28 & 0.01 \\
\hline 10 & 28 & 9.33 & 6.01 & 2.04 & 0.00 & 4.37 & 0.01 \\
\hline 10 & 28 & 9.30 & 6.01 & 2.04 & 0.01 & 4.35 & 0.01 \\
\hline 10 & 56 & 9.43 & 6.01 & 2.12 & 0.02 & 4.88 & 0.01 \\
\hline 10 & 56 & 9.43 & 6.01 & 2.10 & 0.02 & 4.81 & 0.01 \\
\hline 10 & 56 & 9.47 & 6.01 & 2.12 & 0.01 & 4.87 & 0.01 \\
\hline 10 & 91 & 9.31 & 6.01 & 2.10 & 0.00 & 5.28 & 0.01 \\
\hline 10 & 91 & 9.38 & 6.01 & 2.10 & 0.00 & 5.33 & 0.01 \\
\hline 10 & 91 & 9.30 & 6.01 & 2.07 & 0.00 & 5.26 & 0.01 \\
\hline 10 & 182 & 9.42 & 6.01 & 2.11 & -0.01 & 5.86 & 0.01 \\
\hline 10 & 182 & 9.43 & 6.01 & 2.09 & -0.01 & 5.72 & 0.01 \\
\hline 10 & 182 & 9.48 & 6.01 & 2.19 & 0.00 & 6.07 & 0.01 \\
\hline 10 & 364 & 8.64 & 6.00 & 1.76 & -0.01 & 6.60 & 0.01 \\
\hline 10 & 364 & 8.76 & 6.00 & 1.95 & 0.01 & 6.94 & 0.01 \\
\hline 10 & 364 & 8.76 & 6.00 & 1.95 & 0.01 & 6.94 & 0.01 \\
\hline 530 & 0.25 & 9.88 & 5.58 & 1.32 & 0.01 & 3.05 & 0.00 \\
\hline 530 & 0.25 & 9.90 & 5.58 & 1.79 & 0.01 & 3.34 & 0.00 \\
\hline 530 & 0.25 & 9.93 & 5.58 & 1.77 & 0.02 & 3.56 & 0.00 \\
\hline 530 & 0.5 & 9.95 & 5.88 & 1.68 & 0.02 & 4.19 & 0.00 \\
\hline 530 & 0.5 & 9.97 & 5.88 & 1.76 & 0.00 & 4.25 & 0.00 \\
\hline 530 & 0.5 & 9.96 & 5.88 & 1.86 & 0.00 & 4.27 & 0.00 \\
\hline 530 & 1 & 9.95 & 5.74 & 1.82 & 0.00 & 5.84 & 0.00 \\
\hline 530 & 1 & 9.95 & 5.74 & 1.88 & -0.01 & 5.84 & 0.00 \\
\hline 530 & 1 & 9.95 & 5.74 & 1.81 & -0.01 & 5.68 & 0.00 \\
\hline 530 & 3 & 10.03 & 5.51 & 2.07 & 0.01 & 8.96 & 0.00 \\
\hline 530 & 3 & 9.91 & 5.51 & 2.21 & 0.00 & 9.14 & 0.00 \\
\hline 530 & 3 & 10.04 & 5.51 & 2.15 & 0.01 & 9.01 & 0.00 \\
\hline 530 & 7 & 10.10 & 5.64 & 1.96 & 0.00 & 12.29 & -0.01 \\
\hline 530 & 7 & 10.06 & 5.64 & 2.06 & 0.00 & 12.29 & -0.01 \\
\hline
\end{tabular}


ELEMENTAL CONC. (ppm)FROM 18A FILTERED LEACHATE (ALL TESTS)

\begin{tabular}{|c|c|c|c|c|c|c|c|}
\hline $\mathrm{SA} / \mathrm{V}$ & Duration & & & & & & \\
\hline$(m-1)$ & (days) & pH final & $\mathrm{pH}$ in & $\overline{A L}$ & $\overline{\mathrm{AS}}$ & B & BA \\
\hline 530 & 7 & 10.10 & 5.64 & 2.13 & -0.01 & 11.89 & -0.01 \\
\hline 530 & 14 & 9.99 & 5.69 & 1.85 & -0.02 & 14.80 & 0.00 \\
\hline 530 & 14 & 9.98 & 5.69 & 1.88 & -0.01 & 14.50 & 0.00 \\
\hline 530 & 14 & 10.00 & 5.69 & 1.84 & -0.01 & 14.30 & 0.00 \\
\hline 530 & 28 & 10.24 & 5.55 & 1.72 & 0.01 & 15.68 & 0.00 \\
\hline 530 & 28 & 10.23 & 5.55 & 1.74 & 0.00 & 15.78 & 0.00 \\
\hline 530 & 28 & 10.15 & 5.55 & 1.74 & 0.00 & 15.58 & 0.00 \\
\hline 530 & 56 & 10.11 & 5.61 & 1.27 & 0.00 & 17.18 & 0.00 \\
\hline 530 & 56 & 10.11 & 5.61 & 1.43 & 0.01 & 17.18 & $\overline{0.00}$ \\
\hline 530 & 56 & 10.07 & 5.61 & 1.32 & 0.01 & 17.28 & 0.00 \\
\hline 530 & 91 & 9.86 & 5.61 & 1.52 & 0.00 & 18.94 & -0.01 \\
\hline 530 & 91 & 9.90 & 5.61 & 1.60 & 0.00 & 18.94 & -0.01 \\
\hline 530 & 91 & 9.88 & 5.61 & 1.50 & -0.01 & 18.44 & -0.01 \\
\hline 530 & 182 & 9.98 & 5.61 & 1.27 & 0.00 & 19.40 & 0.00 \\
\hline 530 & 182 & 9.94 & 5.61 & 1.02 & 0.00 & 20.40 & 0.00 \\
\hline 530 & 182 & 9.96 & 5.61 & 1.13 & 0.01 & 20.20 & 0.00 \\
\hline 2000 & 0.25 & 10.16 & 5.58 & 2.13 & 0.00 & 6.24 & 0.00 \\
\hline 2000 & 0.25 & 10.13 & 5.58 & 2.03 & 0.02 & 6.21 & 0.00 \\
\hline 2000 & 0.25 & 10.17 & 5.58 & 1.98 & 0.01 & 6.20 & 0.00 \\
\hline 2000 & 0.5 & 10.21 & 5.88 & 1.94 & 0.00 & 8.41 & 0.00 \\
\hline 2000 & 0.5 & 10.18 & 5.88 & 2.02 & 0.00 & 8.14 & 0.00 \\
\hline 2000 & 0.5 & 10.14 & 5.88 & 2.00 & 0.00 & 7.06 & 0.00 \\
\hline 2000 & 1 & 10.02 & 5.69 & 2.26 & 0.00 & 12.16 & 0.00 \\
\hline 2000 & 1 & 10.09 & 5.69 & 1.92 & -0.01 & 12.76 & 0.00 \\
\hline 2000 & 1 & 10.02 & 5.69 & 2.12 & 0.01 & 13.16 & 0.00 \\
\hline 2000 & 3 & 10.32 & 5.51 & 1.82 & -0.03 & 20.49 & 0.00 \\
\hline 2000 & 3 & 10.30 & 5.51 & 1.98 & -0.02 & 20.49 & 0.00 \\
\hline 2000 & 3 & 10.31 & 5.51 & 2.01 & -0.01 & 20.39 & 0.00 \\
\hline 2000 & 7 & 10.39 & 5.74 & 1.92 & 0.01 & 26.00 & 0.00 \\
\hline 2000 & 7 & 10.37 & 5.74 & 1.94 & 0.01 & 25.90 & 0.00 \\
\hline 2000 & 7 & 10.37 & 5.74 & 2.07 & 0.00 & 25.60 & 0.00 \\
\hline 2000 & 14 & 10.27 & 5.69 & 1.90 & 0.01 & 28.80 & 0.00 \\
\hline 2000 & 14 & 10.30 & 5.69 & 1.68 & 0.01 & 28.80 & 0.00 \\
\hline 2000 & 14 & 10.28 & 5.69 & 1.71 & 0.01 & 28.30 & 0.00 \\
\hline 2000 & 28 & 10.51 & 5.55 & 1.64 & 0.00 & 29.65 & -0.01 \\
\hline 2000 & 28 & 10.56 & 5.55 & 1.71 & 0.00 & 32.05 & -0.01 \\
\hline 2000 & 28 & 10.57 & 5.55 & 1.54 & 0.00 & 31.35 & -0.01 \\
\hline 2000 & 56 & 10.42 & 5.61 & 1.44 & 0.00 & 34.19 & 0.00 \\
\hline 2000 & 56 & 10.45 & 5.61 & 1.33 & 0.01 & 34.49 & 0.00 \\
\hline 2000 & 56 & 10.39 & 5.61 & 1.39 & 0.00 & 33.29 & 0.00 \\
\hline 2000 & 91 & 10.34 & 5.61 & 1.87 & -0.01 & 37.69 & 0.00 \\
\hline 2000 & 91 & 10.23 & 5.61 & 1.58 & 0.00 & 37.49 & 0.00 \\
\hline 2000 & 91 & 10.23 & 5.61 & 1.52 & 0.00 & 35.29 & 0.00 \\
\hline 2000 & 182 & 10.37 & 5.61 & 1.38 & 0.01 & 41.79 & 0.00 \\
\hline 2000 & 182 & 10.36 & 5.61 & 1.37 & 0.02 & 42.99 & 0.00 \\
\hline 2000 & 182 & 10.32 & 5.61 & 1.45 & 0.01 & 40.69 & 0.00 \\
\hline 20000 & 1 & 10.79 & 5.58 & 0.92 & 0.15 & 61.47 & 0.00 \\
\hline
\end{tabular}


ELEMENTAL CONC. (ppm)FROM 18A FILTERED LEACHATE (ALL TESTS)

\begin{tabular}{|r|r|r|r|r|r|r|r|}
\hline SA/V & Duration & & & & & & \\
\hline (m-1) & (days) & pH final & pH in & \multicolumn{1}{c|}{ AL } & \multicolumn{1}{c|}{ AS } & \multicolumn{1}{c|}{ B } & \multicolumn{1}{c|}{ BA } \\
\hline 20000 & 1 & 10.80 & 5.58 & 1.02 & 0.04 & 61.21 & 0.00 \\
\hline 20000 & 1 & 10.76 & 5.58 & 1.28 & 0.01 & 67.99 & 0.00 \\
\hline 20000 & 3 & 10.91 & 5.51 & 0.33 & -0.02 & 103.96 & 0.00 \\
\hline 20000 & 3 & 10.86 & 5.51 & 0.40 & -0.02 & 97.51 & 0.00 \\
\hline 20000 & 3 & 10.90 & 5.51 & 0.43 & -0.02 & 96.96 & 0.00 \\
\hline 20000 & 7 & 10.86 & 5.64 & 0.49 & -0.05 & 116.93 & -0.01 \\
\hline 20000 & 7 & 10.89 & 5.64 & 0.68 & -0.07 & 114.43 & -0.01 \\
\hline 20000 & 7 & 10.92 & 5.64 & 0.49 & -0.03 & 114.84 & -0.01 \\
\hline 20000 & 14 & 10.96 & 5.64 & 0.52 & 0.00 & 133.98 & 0.00 \\
\hline 20000 & 14 & 10.98 & 5.64 & 0.61 & 0.00 & 129.78 & 0.00 \\
\hline 20000 & 14 & 10.85 & 5.64 & 0.58 & 0.00 & 129.48 & 0.00 \\
\hline 20000 & 28 & 11.21 & 5.55 & 0.40 & 0.00 & 150.37 & 0.01 \\
\hline 20000 & 28 & 11.20 & 5.55 & 0.44 & 0.00 & 151.37 & 0.00 \\
\hline 20000 & 28 & 11.18 & 5.55 & 0.64 & 0.02 & 146.17 & 0.00 \\
\hline
\end{tabular}


ELEMENTAL CONC. (ppm)FROM 18A FILTERED LEACHATE (ALL TESTS)

\begin{tabular}{|c|c|c|c|c|c|c|c|}
\hline $\mathrm{SA} / \mathrm{V}$ & Duration & & & & & & \\
\hline$(m-1)$ & (days) & BE & CA & $C D$ & $\mathrm{CE}$ & $\mathrm{CO}$ & CR \\
\hline 10 & 0.25 & 0.00 & 0.02 & 0.00 & 0.01 & 0.00 & 0.00 \\
\hline 10 & 0.25 & 0.00 & 0.03 & 0.00 & 0.01 & 0.00 & 0.00 \\
\hline 10 & 0.25 & 0.00 & 0.03 & 0.00 & 0.00 & 0.00 & 0.00 \\
\hline 10 & 0.5 & 0.00 & 0.21 & 0.00 & 0.00 & 0.00 & 0.00 \\
\hline 10 & 0.5 & 0.00 & 0.29 & 0.00 & 0.00 & 0.00 & 0.00 \\
\hline 10 & 0.5 & 0.00 & 0.30 & 0.00 & 0.00 & 0.00 & 0.00 \\
\hline 10 & 1 & 0.00 & 0.48 & 0.00 & 0.00 & 0.00 & 0.00 \\
\hline 10 & 1 & 0.00 & 0.49 & 0.00 & 0.00 & 0.00 & 0.00 \\
\hline 10 & 1 & 0.00 & 0.44 & 0.00 & 0.00 & 0.00 & 0.00 \\
\hline 10 & 3 & 0.00 & 1.11 & 0.00 & 0.00 & 0.00 & 0.00 \\
\hline 10 & 3 & 0.00 & 1.07 & 0.00 & 0.00 & 0.00 & 0.00 \\
\hline 10 & 3 & 0.00 & 1.05 & 0.00 & 0.00 & 0.00 & 0.00 \\
\hline 10 & 7 & 0.00 & 1.29 & 0.00 & 0.00 & 0.00 & 0.00 \\
\hline 10 & 7 & 0.00 & 1.30 & 0.00 & 0.00 & 0.00 & 0.00 \\
\hline 10 & 7 & 0.00 & 1.27 & 0.00 & 0.00 & 0.00 & 0.00 \\
\hline 10 & 14 & 0.00 & 1.35 & 0.00 & 0.00 & 0.00 & 0.00 \\
\hline 10 & 14 & 0.00 & 1.35 & 0.00 & 0.00 & 0.00 & 0.00 \\
\hline 10 & 14 & 0.00 & 1.36 & 0.00 & 0.00 & 0.00 & 0.00 \\
\hline 10 & 28 & 0.00 & 1.49 & 0.00 & 0.00 & 0.00 & 0.00 \\
\hline 10 & 28 & 0.00 & 1.49 & 0.00 & 0.00 & 0.00 & 0.00 \\
\hline 10 & 28 & 0.00 & 1.50 & 0.00 & 0.00 & 0.00 & 0.00 \\
\hline 10 & 56 & 0.00 & 1.54 & 0.00 & 0.00 & 0.00 & 0.01 \\
\hline 10 & 56 & 0.00 & 1.53 & 0.00 & 0.00 & 0.00 & 0.01 \\
\hline 10 & 56 & 0.00 & 1.51 & 0.00 & 0.00 & 0.00 & 0.00 \\
\hline 10 & 91 & 0.00 & 1.44 & 0.00 & 0.00 & 0.00 & 0.00 \\
\hline 10 & 91 & 0.00 & 1.52 & 0.00 & 0.00 & 0.00 & 0.01 \\
\hline 10 & 91 & 0.00 & 1.44 & 0.00 & 0.00 & 0.00 & 0.01 \\
\hline 10 & 182 & 0.00 & 1.78 & 0.00 & 0.00 & 0.00 & 0.01 \\
\hline 10 & 182 & 0.00 & 1.76 & 0.00 & 0.00 & 0.00 & 0.01 \\
\hline 10 & 182 & 0.00 & 1.82 & 0.00 & 0.00 & 0.00 & 0.01 \\
\hline 10 & 364 & 0.00 & 1.84 & 0.00 & 0.01 & 0.00 & 0.01 \\
\hline 10 & 364 & 0.00 & 1.82 & 0.00 & 0.02 & 0.00 & 0.01 \\
\hline 10 & 364 & 0.00 & 1.82 & 0.00 & 0.02 & 0.00 & 0.01 \\
\hline 530 & 0.25 & 0.00 & \begin{tabular}{l|}
0.37 \\
\end{tabular} & 0.00 & 0.00 & 0.00 & 0.00 \\
\hline 530 & 0.25 & 0.00 & 0.48 & 0.00 & 0.00 & 0.00 & $\overline{0.00}$ \\
\hline 530 & 0.25 & 0.00 & 0.41 & 0.00 & 0.00 & 0.00 & 0.00 \\
\hline 530 & 0.5 & 0.00 & 0.30 & 0.00 & 0.00 & 0.00 & 0.00 \\
\hline 530 & 0.5 & 0.00 & 0.42 & 0.00 & 0.00 & 0.00 & 0.00 \\
\hline 530 & 0.5 & 0.00 & 0.45 & 0.00 & 0.00 & 0.00 & 0.00 \\
\hline 530 & 1 & 0.00 & 0.22 & 0.00 & 0.00 & 0.00 & 0.00 \\
\hline 530 & 1 & 0.00 & 0.25 & 0.00 & 0.00 & 0.00 & 0.00 \\
\hline 530 & 1 & 0.00 & 0.18 & 0.00 & 0.00 & 0.00 & 0.00 \\
\hline 530 & 3 & 0.00 & 0.13 & 0.00 & 0.00 & 0.00 & 0.01 \\
\hline 530 & 3 & 0.00 & 0.12 & 0.00 & 0.00 & 0.00 & 0.01 \\
\hline 530 & 3 & 0.00 & 0.13 & 0.00 & 0.00 & 0.00 & 0.01 \\
\hline 530 & 7 & 0.00 & 0.14 & 0.00 & 0.00 & 0.00 & 0.02 \\
\hline 530 & 7 & 0.00 & 0.15 & 0.00 & 0.00 & 0.00 & 0.02 \\
\hline
\end{tabular}


ELEMENTAL CONC. (ppm)FROM 18A FILTERED LEACHATE (ALL TESTS)

\begin{tabular}{|c|c|c|c|c|c|c|c|}
\hline $\mathrm{SA} / \mathrm{V}$ & Duration & & & & & & \\
\hline$(m-1)$ & (days) & $\mathbf{B E}$ & $\overline{C A}$ & $\overline{C D}$ & $\mathrm{CE}$ & $\mathrm{CO}$ & $\overline{C R}$ \\
\hline 530 & 7 & 0.00 & 0.16 & 0.00 & 0.00 & 0.00 & 0.02 \\
\hline 530 & 14 & 0.00 & 0.18 & 0.10 & 0.00 & 0.00 & 0.02 \\
\hline 530 & 14 & 0.00 & 0.22 & 0.00 & 0.00 & 0.00 & 0.02 \\
\hline 530 & 14 & 0.00 & 0.18 & 0.20 & 0.00 & 0.00 & 0.02 \\
\hline 530 & 28 & 0.00 & 0.29 & 0.00 & 0.00 & 0.00 & 0.03 \\
\hline 530 & 28 & 0.00 & 0.28 & 0.00 & 0.00 & 0.00 & 0.03 \\
\hline 530 & 28 & 0.00 & 0.30 & 0.00 & 0.00 & 0.00 & 0.03 \\
\hline 530 & 56 & 0.00 & 0.18 & 0.00 & 0.00 & 0.00 & 0.04 \\
\hline 530 & 56 & 0.00 & 0.24 & 0.00 & 0.00 & 0.00 & 0.04 \\
\hline 530 & 56 & 0.00 & 0.18 & 0.00 & 0.00 & 0.00 & 0.04 \\
\hline 530 & 91 & 0.00 & 0.34 & 0.00 & 0.00 & 0.00 & $\overline{0.05}$ \\
\hline 530 & 91 & 0.00 & 0.40 & 0.00 & 0.00 & 0.00 & 0.05 \\
\hline 530 & 91 & 0.00 & 0.37 & 0.00 & 0.00 & 0.00 & 0.05 \\
\hline 530 & 182 & 0.00 & 0.51 & 0.00 & 0.00 & 0.00 & 0.06 \\
\hline 530 & 182 & 0.00 & 0.51 & 0.00 & 0.00 & 0.00 & 0.06 \\
\hline 530 & 182 & 0.00 & 0.49 & 0.00 & 0.00 & 0.00 & 0.06 \\
\hline 2000 & 0.25 & 0.00 & 0.04 & 0.00 & 0.00 & 0.00 & 0.00 \\
\hline 2000 & 0.25 & 0.00 & 0.06 & 0.00 & 0.00 & 0.00 & 0.00 \\
\hline 2000 & 0.25 & 0.00 & 0.06 & 0.00 & 0.00 & 0.00 & 0.00 \\
\hline 2000 & 0.5 & 0.00 & 0.07 & 0.00 & 0.00 & 0.00 & 0.00 \\
\hline 2000 & 0.5 & 0.00 & 0.05 & 0.00 & 0.00 & 0.00 & 0.00 \\
\hline 2000 & 0.5 & 0.00 & 0.06 & 0.00 & 0.00 & 0.00 & 0.00 \\
\hline 2000 & 1 & 0.00 & 0.03 & 0.00 & 0.00 & 0.00 & 0.01 \\
\hline 2000 & 1 & 0.00 & 0.02 & 0.00 & 0.00 & 0.00 & 0.01 \\
\hline 2000 & 1 & 0.00 & 0.00 & 0.00 & 0.00 & 0.00 & 0.01 \\
\hline 2000 & 3 & 0.00 & 0.05 & 0.00 & 0.00 & 0.00 & 0.01 \\
\hline 2000 & 3 & 0.00 & 0.06 & 0.00 & 0.00 & 0.00 & 0.02 \\
\hline 2000 & 3 & 0.00 & 0.07 & 0.00 & 0.00 & 0.00 & 0.02 \\
\hline 2000 & 7 & 0.00 & 0.17 & 0.00 & 0.00 & 0.00 & 0.04 \\
\hline 2000 & 7 & 0.00 & 0.14 & 0.00 & 0.00 & 0.00 & 0.04 \\
\hline 2000 & 7 & 0.00 & 0.15 & 0.00 & 0.00 & 0.00 & 0.04 \\
\hline 2000 & 14 & 0.00 & 0.17 & 0.20 & 0.00 & 0.00 & 0.05 \\
\hline 2000 & 14 & 0.00 & 0.14 & 0.00 & 0.00 & 0.00 & 0.04 \\
\hline 2000 & 14 & 0.00 & 0.15 & 1.01 & 0.00 & 0.00 & 0.04 \\
\hline 2000 & 28 & 0.00 & 0.20 & 0.00 & 0.00 & 0.00 & 0.06 \\
\hline 2000 & 28 & 0.00 & 0.21 & 0.00 & 0.00 & 0.00 & 0.06 \\
\hline 2000 & 28 & 0.00 & 0.26 & 0.00 & 0.00 & 0.00 & 0.05 \\
\hline 2000 & 56 & 0.00 & 0.32 & 0.00 & 0.00 & 0.00 & 0.09 \\
\hline 2000 & 56 & 0.00 & 0.27 & 0.00 & 0.00 & 0.00 & 0.09 \\
\hline 2000 & 56 & 0.00 & 0.34 & 0.00 & 0.00 & 0.00 & 0.09 \\
\hline 2000 & 91 & 0.00 & 0.58 & 0.00 & 0.00 & 0.00 & 0.13 \\
\hline 2000 & 91 & 0.00 & 1.07 & 0.00 & 0.00 & 0.00 & 0.12 \\
\hline 2000 & 91 & 0.00 & 0.51 & 0.00 & 0.00 & 0.00 & 0.11 \\
\hline 2000 & 182 & 0.00 & 0.69 & 0.00 & 0.00 & 0.00 & 0.16 \\
\hline 2000 & 182 & 0.00 & 0.70 & 0.00 & 0.00 & 0.00 & 0.17 \\
\hline 2000 & 182 & 0.00 & 0.79 & 0.00 & 0.00 & 0.00 & 0.16 \\
\hline 20000 & 1 & 0.00 & 0.07 & 0.00 & 0.00 & 0.00 & 0.01 \\
\hline
\end{tabular}


ELEMENTAL CONC. (ppm)FROM 18A FILTERED LEACHATE (ALL TESTS)

\begin{tabular}{|r|r|r|r|r|r|r|r|}
\hline SA/Y & Duration & & & & & & \\
\hline (m-1) & (days) & \multicolumn{1}{|c|}{ BE } & CA & \multicolumn{1}{c|}{ CD } & \multicolumn{1}{c|}{ CE } & \multicolumn{1}{c|}{ CO } & \multicolumn{1}{c|}{ CR } \\
\hline 20000 & 1 & 0.00 & 0.07 & 0.01 & 0.00 & 0.00 & 0.04 \\
\hline 20000 & 1 & 0.00 & 0.12 & 0.00 & 0.00 & 0.00 & 0.03 \\
\hline 20000 & 3 & 0.00 & 0.06 & 0.00 & 0.00 & 0.00 & 0.18 \\
\hline 20000 & 3 & 0.00 & 0.08 & 0.00 & 0.00 & 0.00 & 0.17 \\
\hline 20000 & 3 & 0.00 & 0.14 & 0.00 & 0.00 & 0.00 & 0.18 \\
\hline 20000 & 7 & 0.00 & -0.51 & 0.00 & -0.08 & -0.03 & 0.32 \\
\hline 20000 & 7 & 0.00 & -0.49 & 0.00 & -0.08 & -0.03 & 0.32 \\
\hline 20000 & 7 & 0.00 & -0.49 & 0.00 & -0.08 & -0.03 & 0.32 \\
\hline 20000 & 14 & 0.00 & -0.16 & 0.00 & 0.00 & 0.00 & 0.42 \\
\hline 20000 & 14 & 0.00 & -0.13 & 0.01 & 0.00 & 0.00 & 0.44 \\
\hline 20000 & 14 & 0.00 & -0.21 & 0.00 & 0.00 & 0.00 & 0.45 \\
\hline 20000 & 28 & 0.00 & 0.44 & 0.00 & 0.00 & 0.00 & 0.45 \\
\hline 20000 & 28 & 0.00 & 0.45 & 0.00 & 0.00 & 0.00 & 0.46 \\
\hline 20000 & 28 & 0.00 & 0.00 & 0.00 & 0.00 & 0.02 & 0.41 \\
\hline
\end{tabular}


ELEMENTAL CONC. (ppm)FROM 18A FILTERED LEACHATE (ALL TESTS)

\begin{tabular}{|c|c|c|c|c|c|c|c|}
\hline $\mathrm{SA} / \mathrm{V}$ & Duration & & & & & & \\
\hline$(m-1)$ & (days) & $\mathrm{CS}$ & $\overline{\mathrm{CU}}$ & $\overline{\mathrm{DY}}$ & $\overline{E U}$ & $\overline{F E}$ & $\overline{G D}$ \\
\hline 10 & 0.25 & 0.00 & 0.00 & 0.00 & 0.00 & 0.00 & 0.00 \\
\hline 10 & 0.25 & 0.00 & 0.00 & 0.00 & 0.00 & 0.00 & 0.00 \\
\hline 10 & 0.25 & 0.00 & 0.00 & 0.00 & 0.00 & 0.00 & 0.00 \\
\hline 10 & 0.5 & 0.01 & 0.00 & 0.00 & 0.00 & 0.00 & 0.00 \\
\hline 10 & 0.5 & 0.02 & 0.00 & 0.00 & 0.00 & 0.00 & 0.00 \\
\hline 10 & 0.5 & 0.02 & 0.00 & 0.00 & 0.00 & 0.00 & 0.00 \\
\hline 10 & 1 & 0.04 & 0.00 & 0.00 & 0.00 & 0.00 & 0.00 \\
\hline 10 & I) & 0.04 & 0.00 & 0.00 & 0.00 & 0.00 & 0.00 \\
\hline 10 & 1 & 0.03 & 0.00 & 0.00 & 0.00 & 0.00 & 0.00 \\
\hline 10 & 3 & 0.12 & 0.00 & 0.00 & 0.00 & 0.00 & -0.01 \\
\hline 10 & 3 & 0.11 & 0.00 & 0.00 & 0.00 & 0.00 & -0.01 \\
\hline 10 & 3 & 0.11 & 0.00 & 0.00 & 0.00 & 0.00 & 0.00 \\
\hline 10 & 7 & 0.16 & 0.00 & 0.00 & 0.00 & 0.00 & -0.01 \\
\hline 10 & 7 & 0.14 & 0.00 & 0.00 & 0.00 & 0.00 & -0.01 \\
\hline 10 & 7 & 0.14 & 0.00 & 0.00 & 0.00 & 0.00 & -0.01 \\
\hline 10 & 14 & 0.15 & 0.00 & 0.00 & 0.00 & 0.00 & 0.00 \\
\hline 10 & 14 & 0.16 & 0.00 & 0.00 & 0.00 & 0.00 & -0.02 \\
\hline 10 & 14 & 0.15 & 0.00 & 0.00 & 0.00 & 0.00 & -0.01 \\
\hline 10 & 28 & 0.17 & 0.00 & 0.00 & 0.00 & 0.00 & -0.01 \\
\hline 10 & 28 & 0.17 & 0.00 & 0.00 & 0.00 & 0.00 & -0.01 \\
\hline 10 & 28 & 0.18 & 0.00 & 0.00 & 0.00 & 0.00 & -0.01 \\
\hline 10 & 56 & 0.20 & 0.01 & 0.00 & 0.00 & 0.00 & -0.01 \\
\hline 10 & 56 & 0.20 & 0.00 & 0.00 & 0.00 & 0.00 & -0.02 \\
\hline 10 & 56 & 0.20 & 0.00 & 0.00 & 0.00 & 0.00 & -0.01 \\
\hline 10 & 91 & 0.23 & 0.00 & 0.00 & 0.00 & 0.00 & -0.02 \\
\hline 10 & 91 & 0.23 & 0.00 & 0.00 & 0.00 & 0.00 & -0.02 \\
\hline 10 & 91 & 0.23 & 0.00 & 0.00 & 0.00 & 0.00 & -0.02 \\
\hline 10 & 182 & 0.25 & 0.00 & 0.00 & 0.00 & 0.00 & -0.02 \\
\hline 10 & 182 & 0.23 & 0.00 & 0.00 & 0.00 & 0.00 & 0.00 \\
\hline 10 & 182 & 0.25 & 0.00 & 0.00 & 0.00 & 0.00 & 0.00 \\
\hline 10 & 364 & 0.28 & 0.01 & 0.00 & 0.00 & 0.00 & -0.01 \\
\hline 10 & 364 & 0.30 & 0.01 & 0.00 & 0.00 & 0.00 & -0.02 \\
\hline 10 & 364 & 0.30 & 0.01 & $\overline{0.00}$ & 0.00 & 0.00 & -0.02 \\
\hline 530 & 0.25 & 0.13 & 0.00 & 0.00 & 0.00 & 0.00 & 0.00 \\
\hline 530 & 0.25 & 0.15 & 0.00 & 0.00 & 0.00 & 0.00 & 0.00 \\
\hline 530 & 0.25 & 0.17 & 0.00 & 0.00 & 0.00 & 0.00 & 0.00 \\
\hline 530 & 0.5 & 0.17 & 0.00 & 0.00 & 0.00 & 0.00 & 0.00 \\
\hline 530 & 0.5 & 0.18 & 0.00 & 0.00 & 0.00 & 0.00 & 0.00 \\
\hline 530 & 0.5 & 0.17 & 0.00 & 0.00 & 0.00 & 0.00 & 0.00 \\
\hline 530 & 1 & 0.20 & 0.00 & 0.00 & 0.00 & 0.00 & -0.01 \\
\hline 530 & 1 & 0.21 & 0.00 & 0.00 & 0.00 & 0.00 & -0.01 \\
\hline 530 & 1 & 0.19 & 0.00 & 0.00 & 0.00 & 0.00 & -0.01 \\
\hline 530 & 3 & 0.25 & 0.00 & 0.00 & 0.00 & 0.00 & -0.01 \\
\hline 530 & 3 & 0.24 & 0.00 & 0.00 & 0.00 & 0.00 & -0.01 \\
\hline 530 & 3 & 0.24 & 0.00 & 0.00 & 0.00 & 0.00 & -0.01 \\
\hline 530 & 7 & 0.26 & 0.00 & 0.00 & 0.00 & 0.00 & -0.02 \\
\hline 530 & 7 & 0.26 & 0.00 & 0.00 & 0.00 & 0.00 & -0.02 \\
\hline
\end{tabular}


ELEMENTAL CONC. (ppm)FROM 18A FILTERED LEACHATE (ALL TESTS)

\begin{tabular}{|c|c|c|c|c|c|c|c|}
\hline SA/V & Duration & & & & & & \\
\hline$(m-1)$ & (days) & $\mathrm{CS}$ & $\mathrm{CU}$ & $\overline{D Y}$ & $\overline{E U}$ & FE & GD \\
\hline 530 & 7 & 0.25 & 0.00 & 0.00 & 0.00 & 0.00 & -0.02 \\
\hline 530 & 14 & 0.23 & 0.00 & 0.00 & 0.00 & 0.00 & -0.02 \\
\hline 530 & 14 & 0.24 & 0.01 & 0.00 & 0.00 & 0.00 & 0.00 \\
\hline 530 & 14 & 0.22 & 0.00 & 0.00 & 0.00 & 0.00 & -0.02 \\
\hline 530 & 28 & 0.22 & 0.00 & 0.00 & 0.00 & 0.00 & -0.02 \\
\hline 530 & 28 & 0.20 & 0.00 & 0.00 & 0.00 & 0.00 & -0.02 \\
\hline 530 & 28 & 0.20 & 0.00 & 0.00 & 0.00 & 0.00 & -0.02 \\
\hline 530 & 56 & 0.19 & 0.00 & 0.00 & 0.00 & 0.00 & -0.02 \\
\hline 530 & 56 & 0.19 & 0.00 & 0.00 & 0.00 & 0.00 & -0.02 \\
\hline 530 & 56 & 0.19 & 0.00 & 0.00 & 0.00 & 0.00 & -0.02 \\
\hline 530 & 91 & 0.22 & 0.00 & 0.00 & 0.00 & 0.00 & -0.02 \\
\hline 530 & 91 & 0.22 & 0.00 & 0.00 & 0.00 & 0.00 & -0.01 \\
\hline 530 & 91 & 0.22 & 0.00 & 0.00 & 0.00 & 0.00 & -0.01 \\
\hline 530 & 182 & 0.25 & 0.00 & 0.00 & 0.00 & 0.00 & -0.02 \\
\hline 530 & 182 & 0.21 & 0.00 & 0.00 & 0.00 & 0.00 & -0.03 \\
\hline 530 & 182 & 0.22 & 0.00 & 0.00 & 0.00 & 0.00 & -0.01 \\
\hline 2000 & 0.25 & 0.23 & 0.00 & 0.00 & 0.00 & 0.00 & 0.00 \\
\hline 2000 & 0.25 & 0.21 & 0.00 & 0.00 & 0.00 & 0.00 & 0.00 \\
\hline 2000 & 0.25 & 0.26 & 0.00 & 0.00 & 0.00 & 0.00 & 0.00 \\
\hline 2000 & 0.5 & 0.20 & 0.00 & 0.00 & 0.00 & 0.00 & 0.00 \\
\hline 2000 & 0.5 & 0.20 & 0.00 & 0.00 & 0.00 & 0.00 & 0.00 \\
\hline 2000 & 0.5 & 0.21 & 0.00 & 0.00 & 0.00 & 0.00 & 0.00 \\
\hline 2000 & 1 & 0.18 & 0.00 & 0.00 & 0.00 & 0.00 & -0.02 \\
\hline 2000 & 1 & 0.16 & 0.00 & 0.00 & 0.00 & 0.00 & -0.02 \\
\hline 2000 & 1 & 0.19 & 0.00 & 0.00 & 0.00 & 0.00 & -0.02 \\
\hline 2000 & 3 & 0.13 & 0.00 & 0.00 & 0.00 & 0.00 & -0.03 \\
\hline 2000 & 3 & 0.17 & 0.00 & 0.00 & 0.00 & 0.00 & -0.03 \\
\hline 2000 & 3 & 0.13 & 0.00 & 0.00 & 0.00 & 0.00 & -0.03 \\
\hline 2000 & 7 & 0.15 & 0.01 & 0.00 & 0.00 & 0.00 & -0.03 \\
\hline 2000 & 7 & 0.15 & 0.01 & 0.00 & 0.00 & 0.00 & -0.02 \\
\hline 2000 & 7 & 0.15 & 0.00 & 0.00 & 0.00 & 0.00 & -0.03 \\
\hline 2000 & 14 & 0.19 & 0.01 & 0.00 & 0.00 & 0.00 & 0.01 \\
\hline 2000 & 14 & 0.15 & 0.00 & 0.00 & 0.00 & 0.00 & -0.01 \\
\hline 2000 & 14 & 0.16 & 0.00 & 0.00 & 0.00 & 0.00 & -0.01 \\
\hline 2000 & 28 & 0.15 & 0.00 & 0.00 & 0.00 & 0.00 & -0.04 \\
\hline 2000 & 28 & 0.17 & 0.00 & 0.00 & 0.00 & 0.00 & -0.04 \\
\hline 2000 & 28 & 0.15 & 0.00 & 0.00 & 0.00 & 0.00 & -0.04 \\
\hline 2000 & 56 & 0.16 & 0.01 & 0.00 & 0.00 & 0.00 & -0.04 \\
\hline 2000 & 56 & 0.15 & 0.00 & 0.00 & 0.00 & 0.00 & -0.04 \\
\hline 2000 & 56 & 0.14 & 0.01 & 0.00 & 0.00 & 0.00 & -0.04 \\
\hline 2000 & 91 & 0.23 & 0.03 & 0.00 & 0.00 & 0.00 & -0.02 \\
\hline 2000 & 91 & 0.22 & 0.02 & 0.00 & 0.00 & 0.00 & -0.04 \\
\hline 2000 & 91 & 0.22 & 0.01 & 0.00 & 0.00 & 0.00 & -0.04 \\
\hline 2000 & 182 & 0.28 & 0.00 & 0.00 & 0.00 & 0.00 & -0.01 \\
\hline 2000 & 182 & 0.30 & 0.00 & 0.00 & 0.00 & 0.00 & -0.02 \\
\hline 2000 & 182 & 0.29 & 0.02 & 0.00 & 0.00 & 0.00 & 0.00 \\
\hline 20000 & 1 & 0.16 & 0.03 & 0.00 & 0.00 & 0.00 & 0.00 \\
\hline
\end{tabular}


ELEMENTAL CONC. (ppm)FROM 18A FILTERED LEACHATE (ALL TESTS)

\begin{tabular}{|r|r|r|r|r|r|r|r|}
\hline SA/V & Duration & & & & & & \\
\hline (m-1) & (days) & \multicolumn{1}{c|}{ CS } & \multicolumn{1}{c|}{ CU } & \multicolumn{1}{c|}{ DY } & \multicolumn{1}{c|}{ EU } & \multicolumn{1}{c|}{ FE } & \multicolumn{1}{c|}{ GD } \\
\hline 20000 & 1 & 0.16 & 0.02 & 0.00 & 0.00 & 0.00 & 0.00 \\
\hline 20000 & 1 & 0.18 & 0.05 & 0.00 & 0.00 & 0.00 & 0.00 \\
\hline 20000 & 3 & 0.20 & 0.00 & 0.00 & 0.00 & 0.00 & -0.10 \\
\hline 20000 & 3 & 0.20 & 0.03 & 0.00 & 0.00 & 0.00 & -0.13 \\
\hline 20000 & 3 & 0.17 & 0.00 & 0.00 & 0.00 & 0.00 & -0.10 \\
\hline 20000 & 7 & 0.22 & -0.02 & -0.02 & -0.01 & -0.01 & -0.26 \\
\hline 20000 & 7 & 0.30 & 0.06 & -0.02 & -0.01 & -0.01 & -0.21 \\
\hline 20000 & 7 & 0.27 & -0.01 & -0.02 & -0.01 & -0.01 & -0.21 \\
\hline 20000 & 14 & 0.23 & 0.00 & 0.00 & 0.00 & 0.00 & -0.15 \\
\hline 20000 & 14 & 0.20 & 0.00 & 0.00 & 0.00 & 0.00 & -0.15 \\
\hline 20000 & 14 & 0.18 & 0.00 & 0.00 & 0.00 & 0.00 & -0.15 \\
\hline 20000 & 28 & 0.13 & 0.00 & 0.00 & 0.00 & 0.00 & -0.15 \\
\hline 20000 & 28 & 0.13 & 0.00 & 0.00 & 0.00 & 0.00 & -0.15 \\
\hline 20000 & 28 & 0.11 & 0.00 & 0.01 & 0.00 & 0.00 & -0.11 \\
\hline
\end{tabular}


ELEMENTAL CONC. (ppm)FROM 18A FILTERED LEACHATE (ALL TESTS)

\begin{tabular}{|c|c|c|c|c|c|c|c|}
\hline $\mathrm{SA} / \mathrm{V}$ & Duration & & & & & & \\
\hline$(m-1)$ & (days) & $\mathbf{K}$ & LA & LI & MG & $\mathrm{MN}$ & $\mathrm{MO}$ \\
\hline 10 & 0.25 & 0.01 & 0.00 & 0.05 & 0.00 & 0.00 & 0.01 \\
\hline 10 & 0.25 & 0.00 & 0.00 & 0.07 & 0.01 & 0.00 & 0.01 \\
\hline 10 & 0.25 & 0.00 & 0.00 & 0.06 & 0.00 & 0.00 & 0.01 \\
\hline 10 & 0.5 & 0.00 & 0.00 & 0.21 & 0.03 & 0.00 & 0.00 \\
\hline 10 & 0.5 & 0.00 & 0.00 & 0.28 & 0.04 & 0.00 & 0.03 \\
\hline 10 & 0.5 & 0.00 & 0.00 & 0.30 & 0.05 & 0.00 & 0.03 \\
\hline 10 & 1 & 0.00 & 0.00 & 0.49 & 0.10 & 0.00 & 0.05 \\
\hline 10 & 1 & 0.00 & 0.00 & 0.49 & 0.10 & 0.00 & 0.05 \\
\hline 10 & 1 & 0.00 & 0.00 & 0.48 & 0.09 & 0.00 & 0.04 \\
\hline 10 & 3 & 0.00 & 0.00 & 1.22 & 0.16 & 0.00 & 0.13 \\
\hline 10 & 3 & 0.00 & 0.00 & 1.11 & 0.20 & 0.00 & 0.13 \\
\hline 10 & 3 & 0.00 & 0.00 & 1.11 & 0.20 & 0.00 & 0.12 \\
\hline 10 & 7 & 0.00 & 0.00 & 1.63 & 0.04 & 0.00 & 0.18 \\
\hline 10 & 7 & 0.00 & 0.00 & 1.66 & 0.04 & 0.00 & 0.18 \\
\hline 10 & 7 & 0.00 & 0.00 & 1.60 & 0.03 & 0.00 & 0.17 \\
\hline 10 & 14 & 0.00 & 0.00 & 1.95 & 0.02 & 0.00 & 0.22 \\
\hline 10 & 14 & 0.00 & 0.00 & 1.99 & 0.01 & 0.00 & 0.22 \\
\hline 10 & 14 & 0.00 & 0.00 & 2.02 & 0.01 & 0.00 & 0.22 \\
\hline 10 & 28 & 0.00 & 0.00 & 2.42 & 0.00 & 0.00 & 0.26 \\
\hline 10 & 28 & 0.00 & 0.00 & 2.49 & 0.02 & 0.00 & 0.26 \\
\hline 10 & 28 & 0.00 & 0.00 & 2.48 & 0.02 & 0.00 & 0.26 \\
\hline 10 & 56 & 0.00 & 0.00 & 2.73 & 0.04 & 0.01 & 0.30 \\
\hline 10 & 56 & 0.00 & 0.00 & 2.73 & 0.03 & 0.01 & 0.29 \\
\hline 10 & 56 & 0.00 & 0.00 & 2.76 & 0.02 & 0.00 & 0.30 \\
\hline 10 & 91 & 0.00 & -0.01 & 2.96 & 0.02 & 0.01 & 0.32 \\
\hline 10 & 91 & 0.00 & -0.01 & 2.95 & 0.02 & 0.01 & 0.33 \\
\hline 10 & 91 & 0.00 & -0.01 & 2.94 & 0.00 & 0.01 & 0.32 \\
\hline 10 & 182 & 0.09 & 0.00 & 3.26 & 0.06 & 0.01 & 0.36 \\
\hline 10 & 182 & 0.18 & 0.00 & 3.19 & 0.08 & 0.01 & 0.35 \\
\hline 10 & 182 & -0.07 & 0.00 & 3.38 & 0.05 & 0.01 & 0.37 \\
\hline 10 & 364 & 0.00 & 0.00 & 3.73 & 0.06 & 0.01 & 0.42 \\
\hline 10 & 364 & 0.00 & 0.01 & 3.96 & 0.08 & 0.01 & 0.43 \\
\hline 10 & 364 & 0.00 & 0.01 & 3.96 & 0.08 & 0.01 & 0.43 \\
\hline 530 & 0.25 & 0.00 & 0.00 & 1.98 & 0.09 & 0.00 & 0.12 \\
\hline 530 & 0.25 & -0.03 & 0.00 & 2.22 & 0.12 & 0.00 & 0.20 \\
\hline 530 & 0.25 & -0.03 & 0.00 & 2.36 & 0.09 & 0.00 & 0.21 \\
\hline 530 & 0.5 & 0.03 & 0.00 & 2.82 & 0.07 & 0.00 & 0.26 \\
\hline 530 & 0.5 & 0.03 & 0.00 & 2.86 & 0.07 & 0.00 & 0.27 \\
\hline 530 & 0.5 & 0.03 & 0.00 & 2.92 & 0.08 & 0.00 & 0.28 \\
\hline 530 & 1 & 0.00 & 0.00 & 3.63 & 0.00 & 0.00 & 0.34 \\
\hline 530 & 1 & 0.00 & 0.00 & 3.61 & 0.00 & 0.00 & 0.35 \\
\hline 530 & 1 & 0.00 & 0.00 & 3.48 & 0.00 & 0.00 & 0.34 \\
\hline 530 & 3 & 0.00 & 0.00 & 5.35 & 0.01 & 0.00 & 0.55 \\
\hline 530 & 3 & 0.00 & 0.00 & 5.41 & 0.01 & 0.00 & 0.56 \\
\hline 530 & 3 & 0.00 & 0.00 & 5.32 & 0.01 & 0.00 & 0.55 \\
\hline 530 & 7 & 0.00 & 0.00 & 7.36 & 0.03 & 0.00 & 0.74 \\
\hline 530 & 7 & 0.00 & 0.00 & 7.35 & 0.00 & 0.00 & 0.74 \\
\hline
\end{tabular}


ELEMENTAL CONC. (ppm)FROM 18A FILTERED LEACHATE (ALL TESTS)

\begin{tabular}{|c|c|c|c|c|c|c|c|}
\hline $\mathrm{SA} / \mathrm{V}$ & Duration & & & & & & \\
\hline$(m-1)$ & (days) & $\mathbf{K}$ & LA & LI & MG & $\mathrm{MN}$ & $\mathrm{MO}$ \\
\hline 530 & 7 & 0.00 & 0.00 & 7.12 & 0.01 & 0.00 & 0.72 \\
\hline 530 & 14 & 0.00 & 0.00 & 8.39 & 0.01 & 0.00 & 0.85 \\
\hline 530 & 14 & 0.00 & 0.00 & 8.21 & 0.06 & 0.01 & 0.83 \\
\hline 530 & 14 & 0.00 & 0.00 & 8.08 & 0.02 & 0.00 & 0.83 \\
\hline 530 & 28 & 0.00 & 0.00 & 9.49 & 0.00 & 0.00 & 0.94 \\
\hline 530 & 28 & 0.00 & 0.00 & 9.46 & 0.00 & 0.00 & 0.94 \\
\hline 530 & 28 & 0.00 & 0.00 & 9.39 & 0.00 & 0.00 & 0.95 \\
\hline 530 & 56 & 0.00 & 0.00 & 10.60 & 0.00 & 0.00 & 1.03 \\
\hline 530 & 56 & 0.00 & 0.00 & 10.70 & 0.02 & 0.00 & 1.04 \\
\hline 530 & 56 & 0.00 & 0.00 & 10.70 & 0.01 & 0.00 & 1.04 \\
\hline 530 & 91 & 0.04 & 0.00 & 11.89 & 0.02 & 0.00 & 1.14 \\
\hline 530 & 91 & 0.00 & 0.00 & 11.99 & 0.00 & 0.00 & 1.14 \\
\hline 530 & 91 & 0.00 & 0.00 & 11.69 & -0.01 & 0.00 & 1.12 \\
\hline 530 & 182 & 0.00 & 0.00 & 12.50 & 0.02 & 0.00 & 1.15 \\
\hline 530 & 182 & 0.00 & 0.00 & 13.30 & 0.00 & 0.00 & 1.16 \\
\hline 530 & 182 & 0.00 & 0.00 & 13.20 & 0.02 & 0.00 & 1.18 \\
\hline 2000 & 0.25 & 0.02 & 0.00 & 4.86 & 0.01 & 0.00 & 0.40 \\
\hline 2000 & 0.25 & 0.00 & 0.00 & 4.77 & 0.03 & 0.00 & 0.39 \\
\hline 2000 & 0.25 & 0.03 & 0.00 & 4.73 & 0.01 & 0.00 & 0.39 \\
\hline 2000 & 0.5 & 0.08 & 0.00 & 5.99 & -0.01 & 0.00 & 0.49 \\
\hline 2000 & 0.5 & 0.03 & 0.00 & 5.76 & -0.01 & 0.00 & 0.47 \\
\hline 2000 & 0.5 & 0.06 & 0.00 & 5.04 & 0.01 & 0.00 & 0.41 \\
\hline 2000 & 1 & 0.00 & 0.00 & 8.10 & 0.01 & 0.00 & 0.76 \\
\hline 2000 & 1 & 0.00 & 0.00 & 8.39 & 0.00 & 0.00 & 0.77 \\
\hline 2000 & 1 & 0.00 & 0.00 & 8.69 & 0.00 & 0.00 & 0.81 \\
\hline 2000 & 3 & 0.00 & 0.00 & 12.69 & -0.01 & 0.00 & 1.23 \\
\hline 2000 & 3 & 0.00 & 0.00 & 12.69 & -0.01 & 0.00 & 1.23 \\
\hline 2000 & 3 & 0.00 & 0.00 & 12.59 & 0.01 & 0.00 & 1.26 \\
\hline 2000 & 7 & 0.00 & 0.00 & 15.80 & 0.03 & 0.00 & 1.48 \\
\hline 2000 & 7 & 0.00 & 0.00 & 15.80 & 0.01 & 0.00 & 1.49 \\
\hline 2000 & 7 & 0.00 & 0.00 & 15.50 & 0.01 & 0.00 & 1.47 \\
\hline 2000 & 14 & 0.00 & 0.00 & 17.40 & 0.03 & 0.00 & 1.65 \\
\hline 2000 & 14 & 0.00 & 0.00 & 17.40 & 0.02 & 0.00 & 1.63 \\
\hline 2000 & 14 & 0.00 & 0.00 & 17.00 & 0.00 & 0.00 & 1.61 \\
\hline 2000 & 28 & 0.00 & 0.00 & 19.00 & 0.00 & 0.00 & 1.73 \\
\hline 2000 & 28 & 0.00 & 0.00 & 20.80 & 0.00 & 0.00 & 1.84 \\
\hline 2000 & 28 & 0.00 & 0.00 & 20.30 & 0.00 & 0.01 & 1.73 \\
\hline 2000 & 56 & 0.00 & 0.00 & 22.60 & 0.05 & 0.01 & 1.93 \\
\hline 2000 & 56 & 0.00 & 0.00 & 22.90 & 0.01 & 0.00 & 1.95 \\
\hline 2000 & 56 & 0.00 & 0.00 & 21.90 & 0.06 & 0.01 & 1.89 \\
\hline 2000 & 91 & 0.00 & 0.03 & 25.50 & 0.16 & 0.03 & 2.17 \\
\hline 2000 & 91 & 0.00 & 0.01 & 25.10 & 0.08 & 0.01 & 2.15 \\
\hline 2000 & 91 & 0.00 & 0.01 & 23.80 & 0.07 & 0.01 & 2.03 \\
\hline 2000 & 182 & 0.00 & 0.00 & 29.00 & 0.03 & 0.00 & 2.46 \\
\hline 2000 & 182 & 0.00 & 0.00 & 29.70 & 0.02 & 0.00 & 2.53 \\
\hline 2000 & 182 & 0.00 & 0.02 & 28.40 & 0.10 & 0.02 & 2.39 \\
\hline 20000 & 1 & 0.00 & 0.00 & 41.10 & 0.09 & -0.01 & 3.49 \\
\hline
\end{tabular}


ELEMENTAL CONC. (ppm)FROM 18A FILTERED LEACHATE (ALL TESTS)

\begin{tabular}{|r|r|r|r|r|r|r|r|}
\hline SA/V & Duration & & & & & & \\
\hline (m-1) & (days) & \multicolumn{1}{c|}{ K } & LA & LI & MG & MN & \multicolumn{1}{c|}{ MO } \\
\hline 20000 & 1 & 0.00 & 0.00 & 42.25 & 0.12 & 0.00 & 3.47 \\
\hline 20000 & 1 & 0.00 & 0.00 & 47.31 & 0.20 & 0.00 & 3.90 \\
\hline 20000 & 3 & 0.00 & 0.00 & 62.47 & -0.01 & 0.00 & 5.70 \\
\hline 20000 & 3 & 0.00 & 0.00 & 60.04 & -0.01 & 0.00 & 5.36 \\
\hline 20000 & 3 & 0.00 & 0.00 & 59.47 & -0.01 & 0.01 & 5.35 \\
\hline 20000 & 7 & -0.98 & -0.02 & 71.40 & -0.21 & 0.00 & 6.38 \\
\hline 20000 & 7 & -0.98 & -0.02 & 69.90 & -0.05 & 0.04 & 6.18 \\
\hline 20000 & 7 & -0.98 & -0.02 & 70.18 & -0.21 & 0.00 & 6.18 \\
\hline 20000 & 14 & 0.00 & 0.00 & 82.00 & 0.00 & 0.00 & 6.70 \\
\hline 20000 & 14 & 0.00 & 0.00 & 79.40 & 0.00 & 0.00 & 6.62 \\
\hline 20000 & 14 & 0.00 & 0.00 & 79.00 & 0.00 & 0.00 & 6.45 \\
\hline 20000 & 28 & 0.00 & -0.01 & 88.88 & 0.04 & 0.04 & 8.30 \\
\hline 20000 & 28 & 0.00 & 0.00 & 89.38 & 0.06 & 0.01 & 8.25 \\
\hline 20000 & 28 & 0.00 & 0.02 & 85.02 & 0.01 & 0.07 & 7.85 \\
\hline
\end{tabular}


ELEMENTAL CONC. (ppm)FROM 18A FILTERED LEACHATE (ALL TESTS)

\begin{tabular}{|c|c|c|c|c|c|c|c|}
\hline $\mathrm{SA} / \mathrm{V}$ & Duration & & & & & & \\
\hline$(m-1)$ & (days) & NA & ND & NI & $\mathbf{P}$ & PB & $\overline{R U}$ \\
\hline 10 & 0.25 & 0.24 & 0.00 & 0.00 & -0.01 & 0.00 & 0.00 \\
\hline 10 & 0.25 & 0.27 & 0.00 & 0.00 & -0.01 & 0.00 & 0.00 \\
\hline 10 & 0.25 & 0.23 & 0.00 & 0.00 & -0.01 & 0.00 & 0.00 \\
\hline 10 & 0.5 & 0.89 & 0.00 & 0.00 & 0.00 & 0.00 & 0.00 \\
\hline 10 & 0.5 & 1.18 & 0.00 & 0.00 & 0.00 & 0.00 & 0.00 \\
\hline 10 & 0.5 & 1.24 & 0.00 & 0.00 & 0.00 & 0.00 & 0.00 \\
\hline 10 & 1 & 2.13 & 0.00 & 0.00 & 0.00 & 0.00 & 0.00 \\
\hline 10 & 1 & 2.17 & 0.00 & 0.00 & 0.00 & 0.00 & 0.00 \\
\hline 10 & 1 & 1.90 & 0.00 & 0.00 & 0.00 & 0.00 & 0.00 \\
\hline 10 & 3 & 5.10 & 0.00 & 0.00 & 0.00 & 0.00 & 0.00 \\
\hline 10 & 3 & 4.74 & 0.00 & 0.01 & 0.00 & 0.00 & 0.00 \\
\hline 10 & 3. & 4.78 & 0.00 & 0.00 & -0.02 & 0.00 & 0.00 \\
\hline 10 & 7 & 7.02 & 0.00 & 0.00 & -0.01 & -0.02 & 0.00 \\
\hline 10 & 7 & 7.20 & 0.00 & 0.00 & 0.01 & -0.01 & 0.00 \\
\hline 10 & 7 & 6.97 & 0.00 & 0.00 & -0.01 & -0.02 & 0.00 \\
\hline 10 & 14 & 8.67 & 0.00 & 0.01 & 0.03 & -0.02 & 0.00 \\
\hline 10 & 14 & 8.70 & 0.00 & 0.00 & 0.02 & -0.02 & 0.00 \\
\hline 10 & 14 & 8.73 & 0.00 & 0.00 & 0.01 & -0.03 & 0.00 \\
\hline 10 & 28 & 9.88 & 0.00 & 0.00 & -0.01 & -0.03 & 0.00 \\
\hline 10 & 28 & 10.19 & 0.00 & 0.00 & 0.00 & -0.03 & 0.00 \\
\hline 10 & 28 & 10.09 & 0.00 & 0.00 & -0.01 & -0.03 & 0.00 \\
\hline 10 & 56 & 11.30 & 0.00 & 0.01 & 0.01 & -0.02 & 0.00 \\
\hline 10 & 56 & 11.20 & 0.00 & 0.00 & 0.03 & -0.02 & 0.00 \\
\hline 10 & 56 & 11.40 & 0.00 & 0.01 & 0.00 & -0.03 & 0.00 \\
\hline 10 & 91 & 12.70 & 0.00 & 0.00 & 0.02 & -0.04 & 0.00 \\
\hline 10 & 91 & 12.80 & 0.00 & 0.01 & 0.00 & -0.04 & 0.00 \\
\hline 10 & 91 & 12.60 & 0.00 & 0.00 & 0.00 & -0.04 & 0.00 \\
\hline 10 & 182 & 14.35 & 0.00 & 0.00 & 0.07 & -0.01 & 0.00 \\
\hline 10 & 182 & 13.95 & 0.00 & 0.00 & 0.05 & 0.00 & 0.00 \\
\hline 10 & 182 & 14.75 & 0.00 & 0.00 & 0.04 & -0.01 & -0.01 \\
\hline 10 & 364 & 15.25 & 0.01 & 0.02 & 0.07 & 0.02 & 0.00 \\
\hline 10 & 364 & 16.05 & 0.01 & 0.01 & 0.08 & 0.02 & 0.00 \\
\hline 10 & 364 & 16.05 & 0.01 & 0.01 & 0.08 & 0.02 & 0.00 \\
\hline 530 & 0.25 & 7.84 & 0.00 & 0.00 & 0.01 & 0.00 & 0.01 \\
\hline 530 & 0.25 & 9.16 & 0.00 & -0.01 & -0.01 & -0.04 & 0.00 \\
\hline 530 & 0.25 & 9.62 & 0.00 & -0.01 & 0.02 & -0.02 & 0.00 \\
\hline 530 & 0.5 & 11.34 & 0.00 & 0.00 & 0.02 & 0.00 & 0.00 \\
\hline 530 & 0.5 & 11.40 & 0.00 & 0.00 & 0.01 & 0.00 & 0.01 \\
\hline 530 & 0.5 & 11.62 & 0.00 & 0.00 & 0.02 & -0.01 & 0.01 \\
\hline 530 & 1 & 14.93 & 0.00 & 0.00 & 0.03 & -0.04 & 0.00 \\
\hline 530 & 1 & 15.03 & 0.00 & 0.00 & 0.02 & -0.04 & 0.00 \\
\hline 530 & 1 & 14.53 & 0.00 & 0.00 & 0.02 & -0.04 & 0.00 \\
\hline 530 & 3 & 22.36 & 0.00 & 0.00 & 0.04 & -0.04 & 0.00 \\
\hline 530 & 3 & 22.86 & 0.00 & 0.00 & 0.05 & -0.05 & 0.00 \\
\hline 530 & 3 & 22.56 & 0.00 & 0.00 & 0.03 & -0.04 & 0.00 \\
\hline 530 & 7 & 31.16 & 0.00 & 0.01 & 0.07 & -0.05 & 0.00 \\
\hline 530 & 7 & 31.16 & 0.00 & 0.00 & 0.05 & -0.05 & 0.00 \\
\hline
\end{tabular}


ELEMENTAL CONC. (ppm)FROM 18A FILTERED LEACHATE (ALL TESTS)

\begin{tabular}{|c|c|c|c|c|c|c|c|}
\hline SA/V & Duration & & & & & & \\
\hline$(m-1)$ & (days) & NA & ND & NI & $\mathrm{P}$ & PB & RU \\
\hline 530 & 7 & 30.16 & 0.00 & 0.00 & 0.05 & -0.05 & 0.00 \\
\hline 530 & 14 & 35.83 & 0.00 & 0.00 & 0.07 & -0.07 & 0.00 \\
\hline 530 & 14 & 35.13 & 0.00 & 0.02 & 0.08 & -0.06 & 0.04 \\
\hline 530 & 14 & 34.63 & 0.00 & 0.01 & 0.10 & -0.07 & 0.01 \\
\hline 530 & 28 & 39.15 & 0.00 & 0.00 & 0.07 & -0.01 & 0.00 \\
\hline 530 & 28 & 39.15 & 0.00 & 0.00 & 0.06 & -0.01 & 0.00 \\
\hline 530 & 28 & 38.85 & 0.00 & 0.00 & 0.07 & -0.01 & 0.00 \\
\hline 530 & 56 & 41.69 & 0.00 & 0.00 & 0.07 & -0.06 & 0.00 \\
\hline 530 & 56 & 41.89 & 0.00 & 0.00 & 0.08 & -0.05 & 0.00 \\
\hline 530 & 56 & 42.29 & 0.00 & 0.00 & 0.10 & -0.05 & 0.00 \\
\hline 530 & 91 & 47.84 & 0.00 & 0.00 & 0.10 & -0.05 & 0.00 \\
\hline 530 & 91 & 47.84 & 0.00 & 0.00 & 0.10 & -0.05 & 0.00 \\
\hline 530 & 91 & 46.74 & 0.00 & 0.00 & 0.07 & -0.05 & 0.00 \\
\hline 530 & 182 & 47.98 & 0.00 & 0.00 & 0.08 & 0.01 & 0.00 \\
\hline 530 & 182 & 50.48 & 0.00 & 0.00 & 0.09 & -0.01 & 0.00 \\
\hline 530 & 182 & 49.88 & 0.00 & 0.00 & 0.10 & 0.00 & 0.00 \\
\hline 2000 & 0.25 & 18.36 & 0.00 & -0.01 & 0.08 & 0.00 & 0.00 \\
\hline 2000 & 0.25 & 18.12 & 0.00 & 0.00 & 0.03 & 0.05 & 0.00 \\
\hline 2000 & 0.25 & 18.05 & 0.00 & 0.00 & 0.05 & 0.01 & 0.00 \\
\hline 2000 & 0.5 & 22.98 & 0.00 & 0.01 & 0.01 & 0.00 & 0.00 \\
\hline 2000 & 0.5 & 22.35 & 0.00 & 0.00 & 0.04 & 0.00 & 0.00 \\
\hline 2000 & 0.5 & 19.91 & 0.00 & 0.00 & 0.03 & 0.00 & 0.01 \\
\hline 2000 & 1 & 30.83 & 0.00 & 0.00 & 0.07 & -0.05 & 0.00 \\
\hline 2000 & 1 & 31.93 & 0.00 & 0.00 & 0.05 & -0.06 & 0.00 \\
\hline 2000 & 1 & 32.83 & 0.00 & 0.00 & 0.07 & -0.05 & 0.00 \\
\hline 2000 & 3 & 50.35 & 0.00 & 0.00 & 0.05 & -0.09 & 0.00 \\
\hline 2000 & 3 & 50.45 & 0.00 & 0.00 & 0.05 & -0.09 & 0.00 \\
\hline 2000 & 3 & 49.85 & 0.00 & 0.00 & 0.09 & -0.08 & 0.00 \\
\hline 2000 & 7 & 61.74 & 0.00 & 0.01 & 0.10 & -0.08 & 0.00 \\
\hline 2000 & 7 & 61.54 & 0.00 & 0.01 & 0.13 & -0.08 & 0.00 \\
\hline 2000 & 7 & 60.84 & 0.00 & 0.01 & 0.11 & -0.08 & 0.00 \\
\hline 2000 & 14 & 68.70 & 0.00 & 0.01 & 0.13 & -0.09 & $\overline{0.05}$ \\
\hline 2000 & 14 & 68.50 & 0.00 & 0.01 & 0.13 & -0.09 & 0.00 \\
\hline 2000 & 14 & 67.10 & 0.00 & 0.00 & 0.13 & -0.09 & 0.00 \\
\hline 2000 & 28 & 72.81 & 0.00 & 0.00 & 0.11 & -0.01 & 0.00 \\
\hline 2000 & 28 & 76.51 & 0.00 & 0.01 & 0.08 & -0.01 & 0.00 \\
\hline 2000 & 28 & 74.61 & 0.00 & 0.01 & 0.06 & -0.01 & 0.00 \\
\hline 2000 & 56 & 81.73 & 0.00 & 0.02 & 0.16 & -0.08 & 0.00 \\
\hline 2000 & 56 & 82.63 & 0.00 & 0.00 & 0.17 & -0.09 & 0.00 \\
\hline 2000 & 56 & 79.63 & 0.00 & 0.02 & 0.15 & -0.09 & 0.00 \\
\hline 2000 & 91 & 93.34 & 0.02 & 0.08 & 0.18 & -0.11 & 0.00 \\
\hline 2000 & 91 & 92.64 & 0.00 & 0.04 & 0.19 & -0.11 & 0.00 \\
\hline 2000 & 91 & 87.74 & 0.00 & 0.03 & 0.19 & -0.12 & 0.00 \\
\hline 2000 & 182 & 101.75 & 0.00 & 0.01 & 0.22 & 0.02 & 0.01 \\
\hline 2000 & 182 & 102.75 & 0.00 & 0.00 & 0.22 & 0.02 & 0.00 \\
\hline 2000 & 182 & 98.35 & 0.01 & 0.05 & 0.22 & 0.03 & 0.01 \\
\hline 20000 & 1 & 139.45 & 0.00 & 0.00 & 0.18 & 0.00 & 0.00 \\
\hline
\end{tabular}


ELEMENTAL CONC. (ppm)FROM 18A FILTERED LEACHATE (ALL TESTS)

\begin{tabular}{|r|r|r|r|r|r|r|r|}
\hline SA/V & Duration & & & & & & \\
\hline (m-1) & (days) & \multicolumn{1}{c|}{ NA } & \multicolumn{1}{c|}{ ND } & \multicolumn{1}{c|}{ NI } & \multicolumn{1}{l|}{ P } & \multicolumn{1}{l|}{ PB } & \multicolumn{1}{c|}{ RU } \\
\hline 20000 & 1 & 141.51 & 0.00 & 0.00 & 0.28 & 0.15 & 0.00 \\
\hline 20000 & 1 & 157.98 & 0.00 & 0.03 & 0.23 & -0.11 & 0.00 \\
\hline 20000 & 3 & 226.03 & 0.00 & -0.01 & 0.15 & -0.26 & 0.00 \\
\hline 20000 & 3 & 214.16 & 0.00 & -0.01 & 0.29 & -0.23 & 0.00 \\
\hline 20000 & 3 & 213.03 & 0.00 & -0.01 & 0.22 & -0.21 & 0.00 \\
\hline 20000 & 7 & 255.61 & -0.07 & -0.03 & 0.21 & -0.42 & 0.00 \\
\hline 20000 & 7 & 252.11 & -0.07 & 0.08 & 0.30 & -0.42 & 0.00 \\
\hline 20000 & 7 & 252.53 & -0.07 & -0.04 & 0.24 & -0.42 & 0.00 \\
\hline 20000 & 14 & 287.99 & 0.00 & 0.00 & 0.38 & -0.30 & 0.00 \\
\hline 20000 & 14 & 278.43 & 0.00 & 0.00 & 0.38 & -0.25 & 0.00 \\
\hline 20000 & 14 & 279.49 & 0.00 & 0.00 & 0.40 & -0.30 & 0.00 \\
\hline 20000 & 28 & 313.12 & 0.00 & 0.00 & 0.22 & 0.00 & 0.00 \\
\hline 20000 & 28 & 313.62 & 0.00 & 0.00 & 0.27 & 0.00 & 0.00 \\
\hline 20000 & 28 & 303.82 & 0.00 & 0.00 & 0.51 & 0.00 & 0.02 \\
\hline
\end{tabular}


ELEMENTAL CONC. (ppm)FROM 18A FILTERED LEACHATE (ALL TESTS)

\begin{tabular}{|c|c|c|c|c|c|c|c|}
\hline SA/V & Duration & & & & & & \\
\hline$(m-1)$ & (days) & SB & SI & $\mathrm{SR}$ & $\mathrm{TE}$ & TI & $\mathrm{V}$ \\
\hline 10 & 0.25 & 0.00 & 0.62 & 0.00 & 0.00 & 0.00 & 0.00 \\
\hline 10 & 0.25 & 0.00 & 0.79 & 0.00 & 0.00 & 0.00 & 0.00 \\
\hline 10 & 0.25 & 0.00 & 0.84 & 0.00 & 0.01 & 0.00 & $\overline{0.00}$ \\
\hline 10 & 0.5 & 0.00 & 2.91 & 0.01 & 0.00 & 0.00 & 0.00 \\
\hline 10 & 0.5 & 0.00 & 3.79 & 0.01 & 0.00 & 0.00 & 0.00 \\
\hline 10 & 0.5 & 0.00 & 4.01 & 0.01 & 0.00 & 0.00 & 0.00 \\
\hline 10 & 1 & 0.00 & 6.57 & 0.01 & 0.00 & 0.00 & 0.00 \\
\hline 10 & 1 & 0.00 & 6.85 & 0.02 & 0.00 & 0.00 & 0.00 \\
\hline 10 & $1)$ & 0.00 & 6.26 & 0.01 & 0.00 & 0.00 & 0.00 \\
\hline 10 & 3 & 0.01 & 15.17 & 0.03 & 0.00 & 0.00 & 0.00 \\
\hline 10 & 3 & 0.01 & 14.17 & 0.03 & 0.00 & 0.00 & 0.00 \\
\hline 10 & 3 & 0.00 & 13.97 & 0.03 & 0.00 & 0.00 & 0.00 \\
\hline 10 & 7 & 0.01 & 19.47 & 0.04 & -0.01 & 0.00 & 0.00 \\
\hline 10 & 7 & 0.01 & 19.77 & 0.04 & 0.00 & 0.00 & 0.00 \\
\hline 10 & 7 & 0.01 & 19.07 & 0.04 & -0.01 & 0.00 & 0.00 \\
\hline 10 & 14 & 0.00 & 23.19 & 0.04 & -0.01 & 0.00 & 0.00 \\
\hline 10 & 14 & 0.00 & 23.29 & 0.04 & -0.01 & 0.00 & 0.00 \\
\hline 10 & 14 & 0.00 & 23.39 & 0.04 & -0.02 & 0.00 & 0.00 \\
\hline 10 & 28 & 0.01 & 26.59 & 0.04 & -0.01 & 0.00 & 0.00 \\
\hline 10 & 28 & 0.00 & 27.09 & $0 . \overline{04}$ & -0.01 & 0.00 & 0.00 \\
\hline 10 & 28 & 0.00 & 26.99 & 0.04 & -0.01 & 0.00 & 0.00 \\
\hline 10 & 56 & 0.01 & 29.78 & 0.04 & 0.01 & 0.00 & 0.00 \\
\hline 10 & 56 & 0.01 & 29.28 & 0.04 & 0.01 & 0.00 & 0.00 \\
\hline 10 & 56 & 0.00 & 29.68 & 0.04 & 0.00 & 0.00 & 0.00 \\
\hline 10 & 91 & 0.00 & 31.96 & 0.04 & -0.03 & 0.00 & 0.00 \\
\hline 10 & 91 & 0.00 & 32.16 & 0.04 & -0.03 & 0.00 & 0.00 \\
\hline 10 & 91 & 0.00 & 31.96 & 0.04 & -0.02 & 0.00 & 0.00 \\
\hline 10 & 182 & 0.00 & 35.20 & 0.04 & -0.02 & 0.00 & 0.00 \\
\hline 10 & 182 & 0.00 & 34.50 & 0.04 & -0.01 & 0.00 & 0.00 \\
\hline 10 & 182 & 0.00 & 36.60 & 0.04 & -0.02 & 0.00 & 0.00 \\
\hline 10 & 364 & 0.03 & 39.07 & 0.04 & -0.01 & 0.00 & 0.00 \\
\hline 10 & 364 & 0.00 & 40.97 & 0.04 & -0.02 & 0.00 & 0.00 \\
\hline 10 & 364 & 0.00 & 40.97 & 0.04 & -0.02 & 0.00 & 0.00 \\
\hline 530 & 0.25 & 0.00 & 20.81 & 0.01 & 0.16 & 0.00 & 0.00 \\
\hline 530 & 0.25 & 0.00 & 23.08 & 0.01 & -0.03 & 0.00 & 0.00 \\
\hline 530 & 0.25 & 0.00 & 24.20 & 0.01 & 0.00 & 0.00 & 0.00 \\
\hline 530 & 0.5 & -0.02 & 27.13 & 0.00 & -0.06 & 0.00 & 0.00 \\
\hline 530 & 0.5 & -0.02 & 27.73 & 0.01 & 0.00 & 0.00 & 0.00 \\
\hline 530 & 0.5 & 0.00 & 28.09 & 0.01 & 0.06 & 0.00 & 0.00 \\
\hline 530 & 1 & 0.00 & 32.50 & 0.00 & -0.03 & 0.00 & 0.00 \\
\hline 530 & 1 & 0.00 & 32.50 & 0.00 & -0.02 & 0.00 & 0.00 \\
\hline 530 & 1 & 0.01 & 31.60 & 0.00 & -0.02 & 0.00 & 0.00 \\
\hline 530 & 3 & 0.02 & 45.69 & 0.00 & -0.01 & 0.00 & 0.00 \\
\hline 530 & 3 & 0.02 & 46.89 & 0.00 & -0.02 & 0.00 & 0.00 \\
\hline 530 & 3 & 0.02 & 46.29 & 0.00 & -0.01 & 0.00 & 0.00 \\
\hline 530 & 7 & 0.02 & 56.60 & 0.00 & -0.01 & 0.00 & 0.00 \\
\hline 530 & 7 & 0.02 & 56.70 & 0.00 & -0.02 & 0.00 & 0.00 \\
\hline
\end{tabular}


ELEMENTAL CONC. (ppm)FROM 18A FILTERED LEACHATE (ALL TESTS)

\begin{tabular}{|c|c|c|c|c|c|c|c|}
\hline $\mathrm{SA} / \mathrm{V}$ & Duration & & & & & & \\
\hline$(m-1)$ & (days) & SB & SI & SR & TE & TI & V \\
\hline 530 & 7 & 0.01 & 55.80 & 0.00 & -0.02 & 0.00 & 0.00 \\
\hline 530 & 14 & 0.02 & 65.70 & 0.00 & -0.01 & 0.00 & 0.00 \\
\hline 530 & 14 & 0.02 & 64.80 & 0.00 & -0.02 & 0.00 & 0.00 \\
\hline 530 & 14 & 0.01 & 63.80 & 0.00 & -0.03 & 0.00 & 0.00 \\
\hline 530 & 28 & 0.04 & 68.36 & 0.01 & -0.03 & 0.00 & 0.00 \\
\hline 530 & 28 & 0.02 & 68.16 & 0.01 & -0.04 & 0.00 & 0.00 \\
\hline 530 & 28 & 0.00 & 67.66 & 0.01 & -0.04 & 0.00 & 0.00 \\
\hline 530 & 56 & 0.00 & 69.30 & 0.00 & 0.00 & 0.00 & 0.00 \\
\hline 530 & 56 & 0.00 & 69.50 & 0.00 & 0.01 & 0.00 & 0.00 \\
\hline 530 & 56 & 0.02 & 69.50 & 0.00 & 0.01 & 0.00 & 0.00 \\
\hline 530 & 91 & 0.03 & 77.34 & 0.01 & 0.02 & 0.00 & 0.00 \\
\hline 530 & 91 & 0.03 & 77.24 & 0.01 & 0.02 & 0.00 & 0.00 \\
\hline 530 & 91 & 0.03 & 75.44 & 0.01 & 0.02 & 0.00 & 0.00 \\
\hline 530 & 182 & 0.03 & 80.49 & 0.01 & -0.02 & 0.00 & 0.00 \\
\hline 530 & 182 & 0.02 & 82.49 & 0.01 & -0.03 & 0.00 & 0.00 \\
\hline 530 & 182 & 0.03 & 82.29 & 0.01 & -0.02 & 0.00 & 0.00 \\
\hline 2000 & 0.25 & 0.02 & 37.16 & 0.00 & 0.13 & 0.00 & 0.00 \\
\hline 2000 & 0.25 & 0.01 & 36.71 & 0.00 & 0.13 & 0.00 & 0.00 \\
\hline 2000 & 0.25 & 0.02 & 36.86 & 0.00 & 0.13 & 0.00 & 0.00 \\
\hline 2000 & 0.5 & 0.00 & 44.44 & 0.00 & 0.10 & 0.00 & 0.00 \\
\hline 2000 & 0.5 & -0.01 & 43.96 & 0.00 & 0.00 & 0.00 & 0.00 \\
\hline 2000 & 0.5 & 0.02 & 40.79 & 0.00 & -0.03 & 0.00 & $\overline{0.00}$ \\
\hline 2000 & 1 & 0.01 & 55.37 & 0.00 & 0.02 & 0.00 & 0.00 \\
\hline 2000 & 1 & 0.01 & 55.77 & 0.00 & 0.02 & 0.00 & 0.00 \\
\hline 2000 & 1 & 0.01 & 58.17 & 0.00 & 0.02 & 0.00 & 0.00 \\
\hline 2000 & 3 & 0.01 & 79.49 & 0.00 & -0.02 & 0.00 & 0.00 \\
\hline 2000 & 3 & 0.00 & 79.99 & 0.00 & -0.01 & 0.00 & 0.00 \\
\hline 2000 & 3 & 0.01 & 79.89 & 0.00 & 0.00 & 0.00 & 0.00 \\
\hline 2000 & 7 & 0.02 & 91.50 & 0.00 & 0.02 & 0.00 & 0.00 \\
\hline 2000 & 7 & 0.02 & 91.40 & 0.00 & 0.02 & 0.00 & 0.00 \\
\hline 2000 & 7 & 0.02 & 91.20 & 0.00 & 0.03 & 0.00 & 0.00 \\
\hline 2000 & 14 & 0.03 & 101.00 & 0.00 & 0.04 & 0.00 & 0.00 \\
\hline 2000 & 14 & 0.03 & 100.00 & 0.00 & 0.04 & 0.00 & 0.00 \\
\hline 2000 & 14 & 0.03 & 98.00 & 0.00 & -0.01 & 0.00 & 0.00 \\
\hline 2000 & 28 & 0.04 & 101.95 & 0.00 & 0.02 & 0.00 & 0.00 \\
\hline 2000 & 28 & 0.04 & 107.95 & 0.00 & -0.01 & 0.00 & 0.00 \\
\hline 2000 & 28 & 0.04 & 105.95 & 0.00 & -0.02 & 0.00 & 0.00 \\
\hline 2000 & 56 & 0.02 & 109.00 & 0.00 & 0.03 & 0.00 & 0.00 \\
\hline 2000 & 56 & 0.02 & 109.00 & 0.00 & 0.03 & 0.00 & 0.00 \\
\hline 2000 & 56 & 0.03 & 107.00 & 0.00 & 0.02 & 0.00 & 0.00 \\
\hline 2000 & 91 & 0.02 & 120.98 & 0.01 & -0.01 & 0.00 & 0.00 \\
\hline 2000 & 91 & 0.02 & 119.98 & 0.01 & -0.02 & 0.00 & 0.00 \\
\hline 2000 & 91 & 0.02 & 114.98 & 0.01 & -0.01 & 0.00 & 0.00 \\
\hline 2000 & 182 & 0.07 & 137.00 & 0.01 & -0.05 & 0.00 & 0.01 \\
\hline 2000 & 182 & $\overline{0.06}$ & 140.00 & 0.01 & -0.06 & 0.00 & 0.01 \\
\hline 2000 & 182 & 0.05 & 134.00 & 0.01 & -0.01 & 0.00 & 0.01 \\
\hline 20000 & 1 & 0.07 & 175.67 & 0.00 & 0.51 & 0.00 & 0.00 \\
\hline
\end{tabular}


ELEMENTAL CONC. (ppm)FROM 18A FILTERED LEACHATE (ALL TESTS)

\begin{tabular}{|r|r|r|r|r|r|r|r|}
\hline SA/V & Duration & & & & & & \\
\hline (m-1) & (days) & \multicolumn{1}{c|}{ SB } & \multicolumn{1}{c|}{ SI } & \multicolumn{1}{c|}{ SR } & \multicolumn{1}{c|}{ TE } & \multicolumn{1}{c|}{ TI } & \multicolumn{1}{|c|}{ V } \\
\hline 20000 & 1 & 0.07 & 176.80 & 0.00 & 0.76 & 0.00 & 0.00 \\
\hline 20000 & 1 & -0.01 & 199.45 & 0.00 & -0.16 & 0.00 & 0.00 \\
\hline 20000 & 3 & 0.00 & 220.97 & 0.00 & 0.14 & 0.00 & 0.00 \\
\hline 20000 & 3 & 0.00 & 209.98 & 0.00 & 0.20 & 0.00 & 0.00 \\
\hline 20000 & 3 & 0.00 & 211.47 & 0.00 & 0.17 & 0.00 & 0.00 \\
\hline 20000 & 7 & -0.03 & 236.74 & -0.01 & 0.64 & -0.01 & -0.02 \\
\hline 20000 & 7 & -0.05 & 234.74 & -0.01 & 0.58 & -0.01 & -0.02 \\
\hline 20000 & 7 & -0.03 & 235.72 & -0.01 & 0.54 & -0.01 & -0.02 \\
\hline 20000 & 14 & 0.00 & 268.97 & 0.00 & 0.46 & 0.00 & 0.00 \\
\hline 20000 & 14 & 0.10 & 261.59 & 0.00 & 0.55 & 0.00 & 0.00 \\
\hline 20000 & 14 & 0.00 & 264.97 & 0.00 & 0.30 & 0.00 & 0.00 \\
\hline 20000 & 28 & 0.11 & 289.00 & 0.01 & 0.19 & 0.00 & 0.01 \\
\hline 20000 & 28 & 0.13 & 289.50 & 0.01 & 0.13 & 0.00 & 0.00 \\
\hline 20000 & 28 & 0.14 & 282.70 & 0.00 & 0.33 & 0.00 & 0.00 \\
\hline
\end{tabular}


ELEMENTAL CONC. (ppm)FROM 18A FILTERED LEACHATE (ALL TESTS)

\begin{tabular}{|c|c|c|c|c|}
\hline $\mathrm{SA} / \mathrm{V}$ & Duration & & & \\
\hline$(m-1)$ & (days) & $\mathbf{Y}$ & ZN & ZR \\
\hline 10 & 0.25 & 0.00 & -0.02 & 0.00 \\
\hline 10 & 0.25 & 0.00 & -0.02 & 0.00 \\
\hline 10 & 0.25 & 0.00 & -0.02 & 0.00 \\
\hline 10 & 0.5 & 0.00 & 0.00 & 0.00 \\
\hline 10 & 0.5 & 0.00 & 0.00 & 0.00 \\
\hline 10 & 0.5 & 0.00 & 0.00 & 0.00 \\
\hline 10 & 1 & 0.00 & 0.00 & 0.00 \\
\hline 10 & 1 & 0.00 & 0.00 & 0.00 \\
\hline 10 & 1 & 0.00 & 0.00 & 0.00 \\
\hline 10 & 3 & 0.00 & 0.00 & 0.00 \\
\hline 10 & 3 & 0.00 & 0.00 & 0.00 \\
\hline 10 & 3 & 0.00 & 0.00 & 0.00 \\
\hline 10 & 7 & 0.00 & 0.00 & 0.00 \\
\hline 10 & 7 & 0.00 & 0.00 & 0.00 \\
\hline 10 & 7 & 0.00 & 0.00 & 0.00 \\
\hline 10 & 14 & 0.00 & 0.00 & 0.00 \\
\hline 10 & 14 & 0.00 & 0.00 & 0.00 \\
\hline 10 & 14 & 0.00 & 0.00 & 0.00 \\
\hline 10 & 28 & 0.00 & 0.00 & -0.01 \\
\hline 10 & 28 & 0.00 & 0.00 & -0.01 \\
\hline 10 & 28 & 0.00 & 0.00 & -0.01 \\
\hline 10 & 56 & 0.00 & -0.04 & 0.00 \\
\hline 10 & 56 & 0.00 & -0.01 & 0.00 \\
\hline 10 & 56 & 0.00 & -0.04 & 0.00 \\
\hline 10 & 91 & 0.00 & 0.00 & 0.01 \\
\hline 10 & 91 & 0.00 & 0.00 & 0.01 \\
\hline 10 & 91 & 0.00 & 0.00 & 0.01 \\
\hline 10 & 182 & 0.00 & 0.00 & 0.00 \\
\hline 10 & 182 & 0.00 & 0.00 & 0.00 \\
\hline 10 & 182 & 0.00 & 0.00 & 0.00 \\
\hline 10 & 364 & 0.00 & 0.00 & 0.00 \\
\hline 10 & 364 & 0.00 & 0.00 & 0.00 \\
\hline 10 & 364 & 0.00 & 0.00 & 0.00 \\
\hline 530 & 0.25 & 0.00 & -0.34 & 0.00 \\
\hline 530 & 0.25 & 0.00 & -0.35 & 0.00 \\
\hline 530 & 0.25 & 0.00 & -0.33 & 0.00 \\
\hline 530 & 0.5 & 0.00 & -0.09 & 0.00 \\
\hline 530 & 0.5 & 0.00 & -0.08 & 0.00 \\
\hline 530 & 0.5 & 0.00 & -0.09 & 0.00 \\
\hline 530 & 1 & 0.00 & -0.17 & 0.00 \\
\hline 530 & 1 & 0.00 & -0.19 & 0.00 \\
\hline 530 & 1 & 0.00 & -0.18 & 0.00 \\
\hline 530 & 3 & 0.00 & -0.07 & 0.00 \\
\hline 530 & 3 & 0.00 & 0.02 & 0.01 \\
\hline 530 & 3 & 0.00 & -0.01 & $\overline{0.01}$ \\
\hline 530 & 7 & 0.00 & -0.08 & 0.00 \\
\hline 530 & 7 & 0.00 & -0.04 & 0.00 \\
\hline
\end{tabular}


ELEMENTAL CONC. (ppm)FROM 18A FILTERED LEACHATE (ALL TESTS)

\begin{tabular}{|c|c|c|c|c|}
\hline $\mathrm{SA} / \mathrm{V}$ & Duration & & & \\
\hline$(m-1)$ & (days) & $\mathbf{Y}$ & $\mathrm{ZN}$ & ZR \\
\hline 530 & 7 & 0.00 & -0.07 & 0.00 \\
\hline 530 & 14 & 0.00 & 0.36 & 0.01 \\
\hline 530 & 14 & 0.00 & 0.21 & 0.01 \\
\hline 530 & 14 & 0.00 & 0.20 & 0.00 \\
\hline 530 & 28 & 0.00 & 0.05 & 0.00 \\
\hline 530 & 28 & 0.00 & 0.27 & 0.00 \\
\hline 530 & 28 & 0.00 & 0.19 & 0.00 \\
\hline 530 & 56 & 0.00 & 0.28 & 0.00 \\
\hline 530 & 56 & 0.00 & 0.09 & 0.00 \\
\hline 530 & 56 & 0.00 & 0.15 & 0.00 \\
\hline 530 & 91 & 0.00 & 0.09 & 0.01 \\
\hline 530 & 91 & 0.00 & -0.01 & 0.01 \\
\hline 530 & 91 & 0.00 & 0.02 & 0.00 \\
\hline 530 & 182 & 0.00 & 0.16 & 0.00 \\
\hline 530 & 182 & 0.00 & 0.33 & 0.00 \\
\hline 530 & 182 & 0.00 & 0.38 & 0.00 \\
\hline 2000 & 0.25 & 0.00 & -0.12 & 0.00 \\
\hline 2000 & 0.25 & 0.00 & -0.10 & 0.00 \\
\hline 2000 & 0.25 & 0.00 & -0.04 & 0.00 \\
\hline 2000 & 0.5 & 0.00 & -0.04 & 0.00 \\
\hline 2000 & 0.5 & 0.00 & -0.10 & 0.00 \\
\hline 2000 & 0.5 & 0.00 & -0.15 & 0.00 \\
\hline 2000 & 1 & 0.00 & 0.21 & 0.00 \\
\hline 2000 & 1 & 0.00 & 0.38 & 0.00 \\
\hline 2000 & 1 & 0.00 & 0.26 & 0.00 \\
\hline 2000 & 3 & 0.00 & 0.09 & 0.00 \\
\hline 2000 & 3 & 0.00 & 0.09 & 0.00 \\
\hline 2000 & 3 & 0.00 & 0.06 & 0.01 \\
\hline 2000 & 7 & 0.00 & 0.19 & 0.01 \\
\hline 2000 & 7 & 0.00 & 0.20 & 0.01 \\
\hline 2000 & 7 & 0.00 & 0.16 & 0.01 \\
\hline 2000 & 14 & 0.00 & 0.32 & 0.01 \\
\hline 2000 & 14 & 0.00 & 0.31 & 0.01 \\
\hline 2000 & 14 & 0.00 & 0.35 & 0.00 \\
\hline 2000 & 28 & 0.00 & 0.10 & 0.00 \\
\hline 2000 & 28 & 0.00 & 0.15 & 0.01 \\
\hline 2000 & 28 & 0.00 & 0.21 & 0.01 \\
\hline 2000 & 56 & 0.00 & 0.40 & 0.01 \\
\hline 2000 & 56 & 0.00 & 0.57 & 0.00 \\
\hline 2000 & 56 & 0.00 & 0.54 & 0.01 \\
\hline 2000 & 91 & 0.00 & 0.45 & 0.05 \\
\hline 2000 & 91 & 0.00 & 0.97 & 0.02 \\
\hline 2000 & 91 & 0.00 & 0.94 & 0.03 \\
\hline 2000 & 182 & 0.00 & 0.37 & 0.01 \\
\hline 2000 & 182 & 0.00 & 0.50 & 0.00 \\
\hline 2000 & 182 & 0.00 & 0.75 & 0.02 \\
\hline 20000 & 1 & 0.00 & 0.62 & 0.00 \\
\hline
\end{tabular}


ELEMENTAL CONC. (ppm)FROM 18A FILTERED LEACHATE (ALL TESTS)

\begin{tabular}{|r|r|r|r|r|}
\hline SA/V & Duration & & & \\
\hline (m-1) & (days) & \multicolumn{1}{|c|}{ Y } & \multicolumn{1}{|c|}{ ZN } & \multicolumn{1}{c|}{ ZR } \\
\hline 20000 & 1 & 0.00 & 0.06 & 0.00 \\
\hline 20000 & 1 & 0.00 & 0.23 & 0.00 \\
\hline 20000 & 3 & 0.00 & 1.58 & 0.00 \\
\hline 20000 & 3 & 0.00 & 1.29 & 0.03 \\
\hline 20000 & 3 & 0.00 & 1.53 & 0.04 \\
\hline 20000 & 7 & -0.01 & -0.66 & 0.02 \\
\hline 20000 & 7 & -0.01 & -0.56 & 0.05 \\
\hline 20000 & 7 & -0.01 & -0.56 & 0.04 \\
\hline 20000 & 14 & 0.00 & -0.55 & 0.04 \\
\hline 20000 & 14 & 0.00 & 0.44 & 0.03 \\
\hline 20000 & 14 & 0.00 & 0.64 & 0.01 \\
\hline 20000 & 28 & 0.00 & 1.30 & 0.03 \\
\hline 20000 & 28 & 0.00 & 1.04 & 0.06 \\
\hline 20000 & 28 & 0.00 & 1.11 & 0.04 \\
\hline
\end{tabular}


, 
CONC. (ppm, blank corrected) FOR 0.45u FILTERED LEACHATES (all test)

\begin{tabular}{|c|c|c|c|c|c|c|c|}
\hline SA/V & Duration & & & & & & \\
\hline$(m-1)$ & (days) & pH final & $\mathrm{pH}$ in & $\overline{\mathrm{AL}}$ & AS & B & $\overline{\mathrm{BA}}$ \\
\hline 10 & 0.25 & 7.48 & 5.58 & 0.07 & 0.00 & 0.09 & 0.00 \\
\hline 10 & 0.25 & 7.34 & 5.58 & 0.09 & 0.00 & 0.11 & 0.00 \\
\hline 10 & 0.25 & 7.27 & 5.58 & 0.08 & 0.00 & 0.11 & 0.00 \\
\hline 10 & 0.5 & 8.97 & 5.88 & 0.27 & 0.00 & 0.39 & 0.00 \\
\hline 10 & 0.5 & 9.07 & 5.88 & 0.36 & 0.00 & 0.51 & 0.01 \\
\hline 10 & 0.5 & 9.03 & 5.88 & 0.39 & 0.00 & 0.55 & 0.01 \\
\hline 10 & 1 & 9.52 & 5.67 & 0.66 & -0.01 & 0.92 & 0.01 \\
\hline 10 & 1 & 9.53 & 5.67 & 0.67 & -0.01 & 0.94 & 0.01 \\
\hline 10 & 1 & 9.49 & 5.67 & 0.60 & -0.01 & 0.86 & 0.01 \\
\hline 10 & 3 & 9.68 & 5.37 & 1.41 & -0.01 & 2.17 & 0.01 \\
\hline 10 & 3 & 9.67 & 5.37 & 1.36 & 0.00 & 2.01 & 0.01 \\
\hline 10 & 3 & 9.65 & 5.37 & 1.34 & 0.00 & 1.98 & 0.01 \\
\hline 10 & 7 & 9.55 & 5.74 & 1.67 & 0.01 & 2.89 & 0.01 \\
\hline 10 & 7 & 9.51 & 5.74 & 1.68 & 0.01 & 2.94 & 0.01 \\
\hline 10 & 7 & 9.51 & 5.74 & 1.63 & 0.01 & 2.83 & 0.01 \\
\hline 10 & 14 & 9.72 & 5.74 & 1.85 & -0.01 & 3.52 & 0.01 \\
\hline 10 & 14 & 9.71 & 5.74 & 1.86 & -0.01 & 3.54 & 0.01 \\
\hline 10 & 14 & 9.71 & 5.74 & 1.86 & -0.01 & 3.54 & 0.01 \\
\hline 10 & 28 & 9.34 & 6.01 & 2.04 & 0.01 & 4.28 & 0.01 \\
\hline 10 & 28 & 9.33 & 6.01 & 2.04 & 0.00 & 4.37 & 0.01 \\
\hline 10 & 28 & 9.30 & 6.01 & 2.04 & 0.01 & 4.35 & 0.01 \\
\hline 10 & 56 & 9.43 & 6.01 & 2.12 & 0.02 & 4.88 & 0.01 \\
\hline 10 & 56 & 9.43 & 6.01 & 2.10 & 0.02 & 4.81 & 0.01 \\
\hline 10 & 56 & 9.47 & 6.01 & 2.12 & 0.01 & 4.87 & 0.01 \\
\hline 10 & 91 & 9.31 & 6.01 & 2.10 & 0.00 & 5.28 & 0.01 \\
\hline 10 & 91 & 9.38 & 6.01 & 2.10 & 0.00 & 5.33 & 0.01 \\
\hline 10 & 91 & 9.30 & 6.01 & 2.07 & 0.00 & 5.26 & 0.01 \\
\hline 10 & 182 & 9.42 & 6.01 & 2.11 & -0.01 & 5.86 & 0.01 \\
\hline 10 & 182 & 9.43 & 6.01 & 2.09 & -0.01 & 5.72 & 0.01 \\
\hline 10 & 182 & 9.48 & 6.01 & 2.19 & 0.00 & 6.07 & 0.01 \\
\hline 10 & 364 & 8.64 & 6.00 & 1.76 & -0.01 & 6.60 & 0.01 \\
\hline 10 & 364 & 8.76 & 6.00 & 1.95 & 0.01 & 6.94 & 0.01 \\
\hline 10 & 364 & 8.76 & 6.00 & 1.95 & 0.01 & 6.94 & 0.01 \\
\hline 530 & 0.25 & 9.88 & 5.58 & 1.81 & 0.00 & 3.12 & 0.01 \\
\hline 530 & 0.25 & 9.90 & 5.58 & 1.88 & -0.01 & 3.29 & 0.01 \\
\hline 530 & 0.25 & 9.93 & 5.58 & 2.03 & -0.01 & 3.51 & 0.02 \\
\hline 530 & 0.5 & 9.95 & 5.88 & 2.05 & 0.00 & 4.17 & 0.02 \\
\hline 530 & 0.5 & \begin{tabular}{|c|}
9.97 \\
\end{tabular} & $\begin{array}{l}5.88 \\
\end{array}$ & 2.05 & 0.02 & 4.27 & 0.01 \\
\hline 530 & 0.5 & 9.96 & 5.88 & 2.00 & -0.01 & 4.23 & 0.01 \\
\hline 530 & 1 & 9.95 & 5.74 & 2.38 & -0.01 & 5.74 & 0.02 \\
\hline 530 & 1 & 9.95 & 5.74 & 2.31 & -0.02 & 5.71 & 0.02 \\
\hline 530 & 1 & 9.95 & 5.74 & 2.29 & -0.01 & 5.58 & 0.02 \\
\hline 530 & 3 & 10.03 & 5.51 & 2.99 & 0.02 & 8.80 & 0.02 \\
\hline 530 & 3 & 9.91 & 5.51 & 3.11 & 0.03 & 8.90 & 0.03 \\
\hline 530 & 3 & 10.04 & 5.51 & 3.15 & 0.03 & 8.85 & 0.03 \\
\hline 530 & 7 & 10.10 & 5.64 & 4.14 & 0.02 & 12.09 & 0.05 \\
\hline 530 & 7 & 10.06 & 5.64 & 4.12 & 0.03 & 11.99 & 0.04 \\
\hline
\end{tabular}


CONC. (ppm, blank corrected) FOR 0.45u FILTERED LEACHATES (all test)

\begin{tabular}{|c|c|c|c|c|c|c|c|}
\hline $\mathrm{SA} / \mathrm{V}$ & Duration & & & & & & \\
\hline$(m-1)$ & (days) & pH final & $\mathrm{pH}$ in & $\mathrm{AL}$ & AS & B & BA \\
\hline 530 & 7 & 10.10 & 5.64 & 3.89 & 0.01 & 11.69 & 0.04 \\
\hline 530 & 14 & 9.99 & 5.69 & 4.72 & 0.03 & 14.19 & 0.06 \\
\hline 530 & 14 & 9.98 & 5.69 & 4.50 & 0.02 & 14.09 & 0.05 \\
\hline 530 & 14 & 10.00 & 5.69 & 4.64 & 0.03 & 14.09 & 0.06 \\
\hline 530 & 28 & 10.24 & 5.55 & 5.66 & -0.01 & 15.47 & 0.08 \\
\hline 530 & 28 & 10.23 & 5.55 & 5.74 & 0.02 & 15.47 & 0.08 \\
\hline 530 & 28 & 10.15 & 5.55 & 5.56 & 0.00 & 15.47 & 0.07 \\
\hline 530 & 56 & 10.11 & 5.61 & 5.18 & 0.03 & 17.00 & 0.06 \\
\hline 530 & 56 & 10.11 & 5.61 & 3.62 & 0.02 & 16.90 & 0.04 \\
\hline 530 & 56 & 10.07 & 5.61 & 5.67 & 0.02 & 17.20 & 0.07 \\
\hline 530 & 91 & 9.86 & 5.61 & 5.03 & 0.03 & 18.19 & 0.05 \\
\hline 530 & 91 & 9.90 & 5.61 & 5.64 & 0.03 & 18.29 & 0.06 \\
\hline 530 & 91 & 9.88 & 5.61 & 5.60 & 0.04 & 18.19 & 0.06 \\
\hline 530 & 182 & 9.98 & 5.61 & 5.81 & 0.02 & 19.20 & 0.07 \\
\hline 530 & 182 & 9.94 & 5.61 & 6.02 & 0.00 & 20.00 & 0.08 \\
\hline 530 & 182 & 9.96 & 5.61 & 5.91 & 0.01 & 20.10 & 0.07 \\
\hline 2000 & 0.25 & 10.16 & 5.58 & 2.29 & 0.00 & 6.14 & 0.01 \\
\hline 2000 & 0.25 & 10.13 & 5.58 & 2.23 & 0.01 & 6.10 & 0.01 \\
\hline 2000 & 0.25 & 10.17 & 5.58 & 2.33 & 0.00 & 6.15 & 0.01 \\
\hline 2000 & 0.5 & 10.21 & 5.88 & 2.45 & 0.01 & 8.30 & 0.01 \\
\hline 2000 & 0.5 & 10.18 & 5.88 & 2.50 & 0.00 & 8.17 & 0.01 \\
\hline 2000 & 0.5 & 10.14 & 5.88 & 2.32 & 0.01 & 6.93 & 0.01 \\
\hline 2000 & 1 & 10.02 & 5.69 & 2.78 & 0.02 & 12.20 & 0.01 \\
\hline 2000 & 1 & 10.09 & 5.69 & 2.81 & 0.02 & 12.60 & 0.01 \\
\hline 2000 & 1 & 10.02 & \begin{tabular}{|l|}
5.69 \\
\end{tabular} & 2.93 & 0.02 & 13.00 & 0.01 \\
\hline 2000 & 3 & 10.32 & 5.51 & 3.87 & -0.01 & 20.10 & 0.03 \\
\hline 2000 & 3 & 10.30 & 5.51 & 3.74 & -0.01 & 20.10 & 0.02 \\
\hline 2000 & 3 & 10.31 & 5.51 & 3.74 & 0.01 & 19.90 & 0.02 \\
\hline 2000 & 7 & 10.39 & 5.74 & 5.68 & 0.04 & 25.40 & 0.04 \\
\hline 2000 & 7 & 10.37 & 5.74 & 5.38 & 0.04 & 25.20 & 0.03 \\
\hline 2000 & 7 & 10.37 & 5.74 & 5.56 & 0.04 & 25.10 & 0.03 \\
\hline 2000 & 14 & 10.27 & 5.69 & 6.53 & 0.04 & 28.49 & $\overline{0.04}$ \\
\hline 2000 & 14 & 10.30 & 5.69 & 6.34 & 0.04 & 28.29 & 0.04 \\
\hline 2000 & 14 & 10.28 & 5.69 & 6.50 & 0.02 & 27.79 & 0.05 \\
\hline 2000 & 28 & 10.51 & 5.55 & 7.22 & 0.04 & 29.35 & 0.05 \\
\hline 2000 & 28 & 10.56 & 5.55 & 7.65 & 0.01 & 31.75 & 0.07 \\
\hline 2000 & 28 & 10.57 & 5.55 & 7.73 & 0.02 & 31.25 & 0.08 \\
\hline 2000 & 56 & 10.42 & 5.61 & 7.89 & 0.05 & 33.78 & 0.07 \\
\hline 2000 & 56 & 10.45 & 5.61 & 7.82 & 0.04 & 34.08 & 0.07 \\
\hline 2000 & 56 & 10.39 & 5.61 & 7.84 & 0.05 & 32.88 & 0.07 \\
\hline 2000 & 91 & 10.34 & 5.61 & 10.10 & 0.05 & 36.70 & 0.10 \\
\hline 2000 & 91 & 10.23 & 5.61 & 10.50 & 0.05 & 37.30 & 0.10 \\
\hline 2000 & 91 & 10.23 & 5.61 & 10.20 & 0.07 & 35.60 & 0.09 \\
\hline 2000 & 182 & 10.37 & 5.61 & 12.20 & 0.03 & 40.60 & 0.13 \\
\hline 2000 & 182 & 10.36 & 5.61 & 12.10 & 0.03 & 41.90 & 0.13 \\
\hline 2000 & 182 & 10.32 & 5.61 & 11.90 & 0.04 & 41.10 & 0.13 \\
\hline 20000 & 1 & 10.79 & 5.58 & 3.42 & 0.01 & 61.38 & 0.02 \\
\hline
\end{tabular}


CONC. (ppm, blank corrected) FOR 0.45u FILTERED LEACHATES (all test)

\begin{tabular}{|r|r|r|r|r|r|r|r|}
\hline SA/V & Duration & & & & & & \\
\hline$(\mathrm{m}-1)$ & (days) & pH final & pH in & \multicolumn{1}{c|}{ AL } & \multicolumn{1}{c|}{ AS } & \multicolumn{1}{c|}{ B } & \multicolumn{1}{c|}{ BA } \\
\hline 20000 & 1 & 10.80 & 5.58 & 3.07 & -0.06 & 60.88 & 0.02 \\
\hline 20000 & 1 & 10.76 & 5.58 & 3.49 & 0.11 & 67.63 & 0.02 \\
\hline 20000 & 3 & 10.91 & 5.51 & 7.32 & -0.03 & 103.47 & 0.03 \\
\hline 20000 & 3 & 10.86 & 5.51 & 6.53 & -0.04 & 95.82 & 0.03 \\
\hline 20000 & 3 & 10.90 & 5.51 & 6.62 & -0.07 & 95.47 & 0.03 \\
\hline 20000 & 7 & 10.86 & 5.64 & 11.49 & 0.05 & 114.94 & 0.05 \\
\hline 20000 & 7 & 10.89 & 5.64 & 11.89 & 0.13 & 113.44 & 0.05 \\
\hline 20000 & 7 & 10.92 & 5.64 & 11.69 & 0.07 & 113.31 & 0.05 \\
\hline 20000 & 14 & 10.96 & 5.64 & 15.20 & -0.05 & 132.99 & 0.06 \\
\hline 20000 & 14 & 10.98 & 5.64 & 15.73 & 0.00 & 128.26 & 0.06 \\
\hline 20000 & 14 & 10.85 & 5.64 & 17.15 & 0.00 & 126.99 & 0.07 \\
\hline 20000 & 28 & 11.21 & 5.55 & 0.49 & 0.01 & 150.40 & 0.00 \\
\hline 20000 & 28 & 11.20 & 5.55 & 0.53 & 0.00 & 150.40 & 0.00 \\
\hline 20000 & 28 & 11.18 & 5.55 & 0.55 & 0.00 & 146.20 & -0.01 \\
\hline
\end{tabular}


CONC. (ppm, blank corrected) FOR 0.45u FILTERED LEACHATES (all test)

\begin{tabular}{|c|c|c|c|c|c|c|c|}
\hline SA/V & Duration & & & & & & \\
\hline$(m-1)$ & (days) & $\overline{B E}$ & $\mathrm{CA}$ & $C D$ & $\mathrm{CE}$ & $\mathrm{CO}$ & CR \\
\hline 10 & 0.25 & 0.00 & 0.02 & 0.00 & 0.01 & 0.00 & 0.00 \\
\hline 10 & 0.25 & 0.00 & 0.03 & 0.00 & 0.01 & 0.00 & 0.00 \\
\hline 10 & 0.25 & 0.00 & 0.03 & 0.00 & 0.00 & 0.00 & 0.00 \\
\hline 10 & 0.5 & 0.00 & 0.21 & 0.00 & 0.00 & 0.00 & 0.00 \\
\hline 10 & 0.5 & 0.00 & 0.29 & 0.00 & 0.00 & 0.00 & 0.00 \\
\hline 10 & 0.5 & 0.00 & 0.30 & 0.00 & 0.00 & 0.00 & 0.00 \\
\hline 10 & 1 & 0.00 & 0.48 & 0.00 & 0.00 & 0.00 & 0.00 \\
\hline 10 & 1 & 0.00 & 0.49 & 0.00 & 0.00 & 0.00 & 0.00 \\
\hline 10 & 1 & 0.00 & 0.44 & 0.00 & 0.00 & 0.00 & 0.00 \\
\hline 10 & 3 & 0.00 & 1.11 & 0.00 & 0.00 & 0.00 & 0.00 \\
\hline 10 & 3 & 0.00 & 1.07 & 0.00 & 0.00 & 0.00 & 0.00 \\
\hline 10 & 3 & 0.00 & 1.05 & 0.00 & 0.00 & 0.00 & 0.00 \\
\hline 10 & 7 & 0.00 & 1.29 & 0.00 & 0.00 & 0.00 & 0.00 \\
\hline 10 & 7 & 0.00 & 1.30 & 0.00 & 0.00 & 0.00 & 0.00 \\
\hline 10 & 7 & 0.00 & 1.27 & 0.00 & 0.00 & 0.00 & 0.00 \\
\hline 10 & 14 & 0.00 & 1.35 & 0.00 & 0.00 & 0.00 & 0.00 \\
\hline 10 & 14 & 0.00 & 1.35 & 0.00 & 0.00 & 0.00 & 0.00 \\
\hline 10 & 14 & 0.00 & 1.36 & 0.00 & 0.00 & 0.00 & 0.00 \\
\hline 10 & 28 & 0.00 & 1.49 & 0.00 & 0.00 & 0.00 & 0.00 \\
\hline 10 & 28 & 0.00 & 1.49 & 0.00 & 0.00 & 0.00 & 0.00 \\
\hline 10 & 28 & 0.00 & 1.50 & 0.00 & 0.00 & 0.00 & 0.00 \\
\hline 10 & 56 & 0.00 & 1.54 & 0.00 & 0.00 & 0.00 & 0.01 \\
\hline 10 & 56 & 0.00 & 1.53 & 0.00 & 0.00 & 0.00 & 0.01 \\
\hline 10 & 56 & 0.00 & 1.51 & 0.00 & 0.00 & 0.00 & 0.00 \\
\hline 10 & 91 & 0.00 & 1.44 & 0.00 & 0.00 & 0.00 & 0.00 \\
\hline 10 & 91 & 0.00 & 1.52 & 0.00 & 0.00 & 0.00 & 0.01 \\
\hline 10 & 91 & 0.00 & 1.44 & 0.00 & 0.00 & 0.00 & 0.01 \\
\hline 10 & 182 & 0.00 & 1.78 & 0.00 & 0.00 & 0.00 & 0.01 \\
\hline 10 & 182 & 0.00 & 1.76 & 0.00 & 0.00 & 0.00 & 0.01 \\
\hline 10 & 182 & 0.00 & 1.82 & 0.00 & 0.00 & 0.00 & 0.01 \\
\hline 10 & 364 & 0.00 & 1.84 & 0.00 & 0.01 & 0.00 & 0.01 \\
\hline 10 & 364 & 0.00 & 1.82 & 0.00 & 0.02 & 0.00 & 0.01 \\
\hline 10 & 364 & 0.00 & 1.82 & 0.00 & 0.02 & 0.00 & 0.01 \\
\hline 530 & 0.25 & 0.00 & 0.91 & 0.00 & 0.00 & 0.00 & 0.01 \\
\hline 530 & 0.25 & 0.00 & 0.96 & 0.00 & 0.00 & 0.00 & 0.01 \\
\hline 530 & 0.25 & 0.00 & 0.97 & 0.00 & 0.00 & 0.00 & 0.02 \\
\hline 530 & 0.5 & 0.00 & 0.96 & 0.00 & 0.02 & 0.00 & 0.03 \\
\hline 530 & 0.5 & 0.00 & 0.91 & 0.00 & 0.01 & 0.00 & 0.02 \\
\hline 530 & 0.5 & 0.00 & 0.85 & 0.00 & 0.00 & 0.00 & 0.02 \\
\hline 530 & 1 & 0.00 & 0.98 & 0.00 & 0.03 & 0.00 & 0.07 \\
\hline 530 & 1 & 0.00 & 0.92 & 0.00 & 0.01 & 0.00 & 0.06 \\
\hline 530 & 1 & 0.00 & 0.95 & 0.00 & 0.02 & 0.00 & 0.06 \\
\hline 530 & 3 & 0.00 & 0.76 & 0.00 & 0.04 & 0.00 & 0.04 \\
\hline 530 & 3 & 0.00 & 0.90 & 0.00 & 0.05 & 0.01 & 0.06 \\
\hline 530 & 3 & 0.00 & 1.00 & 0.00 & 0.06 & 0.01 & 0.06 \\
\hline 530 & 7 & 0.00 & 1.45 & 0.00 & 0.04 & 0.00 & 0.07 \\
\hline 530 & 7 & 0.00 & 1.44 & 0.00 & 0.05 & 0.00 & 0.07 \\
\hline
\end{tabular}


CONC. (ppm, blank corrected) FOR 0.45u FILTERED LEACHATES (all test)

\begin{tabular}{|c|c|c|c|c|c|c|c|}
\hline $\mathrm{SA} / \mathrm{V}$ & Duration & & & & & & \\
\hline$(m-1)$ & (days) & BE & CA & $C D$ & CE & $\mathrm{CO}$ & CR \\
\hline 530 & 7 & 0.00 & 1.33 & 0.00 & 0.04 & 0.00 & 0.06 \\
\hline 530 & 14 & 0.00 & 1.99 & 0.50 & 0.07 & 0.00 & 0.08 \\
\hline 530 & 14 & 0.00 & 1.80 & 0.61 & 0.05 & 0.00 & 0.07 \\
\hline 530 & 14 & 0.00 & 1.96 & 0.91 & 0.07 & 0.00 & 0.08 \\
\hline 530 & 28 & 0.00 & 2.76 & 0.00 & 0.10 & 0.00 & 0.12 \\
\hline 530 & 28 & 0.00 & 2.71 & 0.00 & 0.11 & 0.00 & 0.14 \\
\hline 530 & 28 & 0.00 & 2.60 & 0.00 & 0.08 & 0.00 & 0.13 \\
\hline 530 & 56 & 0.00 & 2.47 & 0.00 & 0.07 & 0.01 & 0.11 \\
\hline 530 & 56 & 0.00 & 1.54 & 0.00 & 0.05 & 0.00 & 0.09 \\
\hline 530 & 56 & 0.00 & 2.76 & 0.00 & 0.12 & 0.01 & 0.14 \\
\hline 530 & 91 & 0.00 & 2.33 & 0.00 & 0.09 & 0.01 & 0.13 \\
\hline 530 & 91 & 0.00 & 2.69 & 0.00 & 0.09 & 0.01 & 0.14 \\
\hline 530 & 91 & 0.00 & 2.63 & 0.00 & 0.08 & 0.01 & 0.13 \\
\hline 530 & 182 & 0.00 & 2.98 & 0.00 & 0.07 & 0.00 & 0.17 \\
\hline 530 & 182 & 0.00 & 3.29 & 0.00 & 0.10 & 0.00 & 0.19 \\
\hline 530 & 182 & 0.00 & 3.15 & 0.00 & 0.08 & 0.00 & 0.18 \\
\hline 2000 & 0.25 & 0.00 & 0.25 & 0.00 & 0.00 & 0.00 & 0.01 \\
\hline 2000 & 0.25 & 0.00 & 0.27 & 0.00 & 0.00 & 0.00 & 0.01 \\
\hline 2000 & 0.25 & 0.00 & 0.35 & 0.00 & 0.00 & 0.00 & 0.02 \\
\hline 2000 & 0.5 & 0.00 & 0.27 & 0.00 & 0.00 & 0.00 & 0.02 \\
\hline 2000 & 0.5 & 0.00 & 0.23 & 0.00 & 0.00 & 0.00 & 0.01 \\
\hline 2000 & 0.5 & 0.00 & 0.28 & 0.00 & 0.00 & 0.00 & 0.01 \\
\hline 2000 & 1 & 0.00 & 0.31 & 0.00 & 0.03 & 0.01 & 0.04 \\
\hline 2000 & 1 & 0.00 & 0.32 & 0.00 & 0.03 & 0.01 & 0.04 \\
\hline 2000 & 1 & 0.00 & 0.37 & 0.00 & 0.04 & 0.01 & $\overline{0.04}$ \\
\hline 2000 & 3 & 0.00 & 0.98 & 0.00 & 0.04 & 0.00 & 0.08 \\
\hline 2000 & 3 & 0.00 & 0.80 & 0.00 & 0.02 & 0.00 & $0 . \overline{08}$ \\
\hline 2000 & 3 & 0.00 & 0.92 & 0.00 & 0.03 & 0.00 & 0.08 \\
\hline 2000 & 7 & 0.00 & 1.76 & 0.00 & 0.13 & 0.02 & 0.16 \\
\hline 2000 & 7 & 0.00 & 1.54 & 0.00 & 0.11 & 0.02 & 0.14 \\
\hline 2000 & 7 & 0.00 & 1.62 & 0.00 & 0.10 & 0.02 & 0.14 \\
\hline 2000 & 14 & 0.00 & 2.01 & 1.81 & 0.14 & 0.01 & 0.17 \\
\hline 2000 & 14 & 0.00 & 1.96 & 1.41 & 0.13 & 0.01 & 0.16 \\
\hline 2000 & 14 & 0.00 & 2.13 & 1.71 & 0.13 & 0.01 & 0.18 \\
\hline 2000 & 28 & 0.00 & 2.45 & 0.00 & 0.09 & 0.00 & 0.19 \\
\hline 2000 & 28 & 0.00 & 2.72 & 0.00 & 0.15 & 0.01 & 0.20 \\
\hline 2000 & 28 & 0.00 & 3.00 & 0.00 & 0.17 & 0.01 & 0.20 \\
\hline 2000 & 56 & 0.00 & 3.07 & 0.00 & 0.24 & 0.02 & 0.31 \\
\hline 2000 & 56 & 0.00 & 2.99 & 0.00 & 0.24 & 0.02 & 0.31 \\
\hline 2000 & 56 & 0.00 & 3.26 & 0.00 & 0.26 & 0.02 & 0.31 \\
\hline 2000 & 91 & 0.00 & 4.26 & 0.00 & 0.34 & 0.02 & 0.42 \\
\hline 2000 & 91 & 0.00 & 4.58 & 0.00 & 0.35 & 0.02 & 0.43 \\
\hline 2000 & 91 & 0.00 & 4.43 & 0.00 & 0.33 & 0.03 & 0.42 \\
\hline 2000 & 182 & 0.00 & 5.79 & 0.00 & 0.49 & 0.02 & 0.62 \\
\hline 2000 & 182 & 0.00 & 6.16 & 0.00 & 0.53 & 0.02 & 0.60 \\
\hline 2000 & 182 & 0.00 & 6.23 & 0.00 & 0.52 & 0.02 & 0.58 \\
\hline 20000 & 1 & 0.00 & 0.90 & 0.01 & 0.00 & 0.00 & 0.08 \\
\hline
\end{tabular}


CONC. (ppm, blank corrected) FOR 0.45u FILTERED LEACHATES (all test)

\begin{tabular}{|r|r|r|r|r|r|r|r|}
\hline SA/V & Duration & & & & & & \\
\hline$(\mathrm{m}-1)$ & (days) & BE & \multicolumn{1}{c|}{ CA } & \multicolumn{1}{c|}{ CD } & \multicolumn{1}{c|}{ CE } & \multicolumn{1}{c|}{ CO } & \multicolumn{1}{c|}{ CR } \\
\hline 20000 & 1 & 0.00 & 0.81 & 0.01 & 0.00 & 0.00 & 0.09 \\
\hline 20000 & 1 & 0.00 & 0.94 & 0.01 & 0.00 & 0.00 & 0.12 \\
\hline 20000 & 3 & 0.00 & 2.08 & 0.00 & 0.09 & 0.09 & 0.40 \\
\hline 20000 & 3 & 0.00 & 1.85 & 0.00 & -0.04 & 0.08 & 0.37 \\
\hline 20000 & 3 & 0.00 & 1.95 & 0.00 & 0.02 & 0.09 & 0.40 \\
\hline 20000 & 7 & 0.00 & 2.98 & 0.00 & 0.31 & 0.10 & 0.73 \\
\hline 20000 & 7 & 0.00 & 2.98 & 0.00 & 0.36 & 0.12 & 0.75 \\
\hline 20000 & 7 & 0.00 & 2.91 & 0.00 & 0.30 & 0.11 & 0.71 \\
\hline 20000 & 14 & 0.00 & 3.04 & 0.01 & 0.32 & 0.12 & 1.10 \\
\hline 20000 & 14 & 0.00 & 3.13 & 0.01 & 0.35 & 0.11 & 1.13 \\
\hline 20000 & 14 & 0.00 & 3.66 & 0.01 & 0.47 & 0.13 & 1.25 \\
\hline 20000 & 28 & 0.00 & 0.40 & 0.00 & 0.00 & 0.01 & 0.49 \\
\hline 20000 & 28 & 0.00 & 0.33 & 0.00 & 0.00 & 0.00 & 0.49 \\
\hline 20000 & 28 & 0.00 & 0.00 & 0.00 & 0.00 & 0.02 & 0.48 \\
\hline
\end{tabular}


CONC. (ppm, blank corrected) FOR 0.45u FILTERED LEACHATES (all test)

\begin{tabular}{|c|c|c|c|c|c|c|c|}
\hline $\mathrm{SA} / \mathrm{V}$ & Duration & & & & & & \\
\hline$(m-1)$ & (days) & $C S$ & $\mathrm{CU}$ & DY & $\overline{E U}$ & FE & $\overline{G D}$ \\
\hline 10 & 0.25 & 0.00 & 0.00 & 0.00 & 0.00 & 0.00 & 0.00 \\
\hline 10 & \begin{tabular}{l|l}
0.25 \\
\end{tabular} & 0.00 & 0.00 & $\begin{array}{l}0.00 \\
\end{array}$ & 0.00 & 0.00 & 0.00 \\
\hline 10 & 0.25 & 0.00 & 0.00 & 0.00 & 0.00 & 0.00 & 0.00 \\
\hline 10 & 0.5 & 0.01 & 0.00 & 0.00 & 0.00 & 0.00 & 0.00 \\
\hline 10 & 0.5 & 0.02 & 0.00 & 0.00 & 0.00 & 0.00 & 0.00 \\
\hline 10 & 0.5 & 0.02 & 0.00 & \begin{tabular}{l|l}
0.00 \\
\end{tabular} & 0.00 & 0.00 & 0.00 \\
\hline 10 & 1 & 0.04 & 0.00 & \begin{tabular}{l|l}
0.00 \\
\end{tabular} & 0.00 & 0.00 & 0.00 \\
\hline 10 & 1 & 0.04 & 0.00 & 0.00 & 0.00 & 0.00 & 0.00 \\
\hline 10 & 1 & 0.03 & 0.00 & 0.00 & 0.00 & 0.00 & 0.00 \\
\hline 10 & 3 & 0.12 & 0.00 & 0.00 & 0.00 & 0.00 & -0.01 \\
\hline 10 & 3 & 0.11 & 0.00 & 0.00 & 0.00 & 0.00 & -0.01 \\
\hline 10 & 3 & 0.11 & 0.00 & 0.00 & 0.00 & 0.00 & 0.00 \\
\hline 10 & \begin{tabular}{l|}
7 \\
\end{tabular} & 0.16 & 0.00 & 0.00 & 0.00 & 0.00 & -0.01 \\
\hline 10 & 7 & 0.14 & 0.00 & 0.00 & 0.00 & 0.00 & -0.01 \\
\hline 10 & 7 & 0.14 & 0.00 & 0.00 & 0.00 & 0.00 & -0.01 \\
\hline 10 & 14 & 0.15 & 0.00 & 0.00 & 0.00 & 0.00 & 0.00 \\
\hline 10 & 14 & 0.16 & 0.00 & 0.00 & 0.00 & 0.00 & -0.02 \\
\hline 10 & 14 & 0.15 & 0.00 & 0.00 & 0.00 & 0.00 & -0.01 \\
\hline 10 & 28 & 0.17 & 0.00 & 0.00 & 0.00 & 0.00 & -0.01 \\
\hline 10 & 28 & 0.17 & 0.00 & 0.00 & 0.00 & 0.00 & -0.01 \\
\hline 10 & 28 & 0.18 & 0.00 & 0.00 & 0.00 & 0.00 & -0.01 \\
\hline 10 & 56 & 0.20 & 0.01 & 0.00 & 0.00 & 0.00 & -0.01 \\
\hline 10 & \begin{tabular}{l|l}
56 \\
\end{tabular} & 0.20 & 0.00 & 0.00 & 0.00 & 0.00 & -0.02 \\
\hline 10 & 56 & 0.20 & 0.00 & 0.00 & 0.00 & 0.00 & -0.01 \\
\hline 10 & 91 & 0.23 & 0.00 & 0.00 & 0.00 & 0.00 & -0.02 \\
\hline 10 & 91 & 0.23 & 0.00 & 0.00 & 0.00 & 0.00 & -0.02 \\
\hline 10 & 91 & 0.23 & 0.00 & \begin{tabular}{l|l}
0.00 \\
\end{tabular} & $0 . \overline{00}$ & 0.00 & -0.02 \\
\hline 10 & 182 & 0.25 & 0.00 & 0.00 & 0.00 & 0.00 & -0.02 \\
\hline 10 & 182 & 0.23 & 0.00 & 0.00 & 0.00 & 0.00 & 0.00 \\
\hline 10 & 182 & 0.25 & 0.00 & 0.00 & 0.00 & 0.00 & 0.00 \\
\hline 10 & 364 & 0.28 & 0.01 & 0.00 & 0.00 & 0.00 & -0.01 \\
\hline 10 & 364 & 0.30 & 0.01 & 0.00 & 0.00 & 0.00 & -0.02 \\
\hline 10 & 364 & 0.30 & 0.01 & 0.00 & 0.00 & 0.00 & -0.02 \\
\hline 530 & 0.25 & 0.16 & 0.03 & 0.00 & 0.00 & 0.00 & 0.00 \\
\hline 530 & 0.25 & 0.17 & 0.04 & 0.00 & 0.00 & 0.00 & 0.00 \\
\hline 530 & 0.25 & 0.18 & 0.04 & 0.00 & 0.00 & 0.00 & 0.00 \\
\hline 530 & 0.5 & 0.20 & 0.06 & 0.00 & 0.00 & 0.00 & 0.00 \\
\hline 530 & 0.5 & 0.19 & 0.05 & 0.00 & 0.00 & 0.00 & 0.00 \\
\hline 530 & 0.5 & 0.19 & 0.04 & 0.00 & 0.00 & 0.00 & 0.00 \\
\hline 530 & 1 & 0.22 & 0.08 & 0.00 & 0.00 & 0.00 & -0.02 \\
\hline 530 & 1 & 0.22 & 0.07 & 0.00 & 0.00 & 0.00 & -0.02 \\
\hline 530 & 1 & 0.21 & 0.07 & 0.00 & 0.00 & 0.00 & -0.02 \\
\hline 530 & 3 & 0.31 & 0.08 & 0.00 & 0.00 & 0.00 & -0.02 \\
\hline 530 & 3 & 0.30 & 0.09 & 0.00 & 0.00 & 0.00 & -0.02 \\
\hline 530 & 3 & 0.31 & $0 . \overline{10}$ & 0.00 & 0.00 & 0.00 & 0.00 \\
\hline 530 & 7 & 0.40 & 0.15 & 0.00 & 0.00 & 0.00 & -0.04 \\
\hline 530 & 7 & 0.42 & 0.15 & 0.00 & 0.00 & 0.00 & -0.04 \\
\hline
\end{tabular}


CONC. (ppm, blank corrected) FOR 0.45u FILTERED LEACHATES (all test)

\begin{tabular}{|c|c|c|c|c|c|c|c|}
\hline SA/V & Duration & & & & & & \\
\hline$(m-1)$ & (days) & $\mathrm{CS}$ & $\mathrm{CU}$ & DY & EU & $\mathrm{FE}$ & GD \\
\hline 530 & 7 & 0.38 & 0.13 & 0.00 & 0.00 & 0.00 & -0.03 \\
\hline 530 & 14 & 0.42 & 0.20 & 0.00 & 0.00 & 0.00 & 0.00 \\
\hline 530 & 14 & 0.41 & 0.17 & 0.00 & 0.00 & 0.00 & -0.01 \\
\hline 530 & 14 & 0.38 & 0.19 & 0.00 & 0.00 & 0.00 & -0.01 \\
\hline 530 & 28 & 0.42 & 0.27 & 0.00 & 0.00 & 0.00 & -0.05 \\
\hline 530 & 28 & 0.40 & 0.27 & 0.00 & 0.00 & 0.00 & -0.07 \\
\hline 530 & 28 & 0.39 & 0.25 & 0.00 & 0.00 & 0.00 & -0.06 \\
\hline 530 & 56 & 0.39 & 0.25 & 0.00 & 0.00 & 0.00 & -0.03 \\
\hline 530 & 56 & 0.29 & 0.15 & 0.00 & 0.00 & 0.00 & -0.03 \\
\hline 530 & 56 & 0.42 & 0.29 & 0.00 & 0.00 & 0.00 & -0.03 \\
\hline 530 & 91 & 0.42 & 0.23 & 0.00 & 0.00 & 0.00 & -0.01 \\
\hline 530 & 91 & 0.44 & 0.28 & 0.00 & 0.00 & 0.00 & -0.03 \\
\hline 530 & \begin{tabular}{|l|}
91 \\
\end{tabular} & 0.46 & 0.27 & 0.00 & 0.00 & 0.00 & -0.04 \\
\hline 530 & 182 & 0.53 & 0.30 & 0.00 & 0.00 & 0.00 & -0.04 \\
\hline 530 & 182 & 0.48 & 0.31 & 0.00 & 0.00 & 0.00 & -0.04 \\
\hline 530 & 182 & 0.47 & 0.31 & 0.00 & 0.00 & 0.00 & -0.05 \\
\hline 2000 & 0.25 & 0.25 & 0.03 & 0.00 & 0.00 & 0.00 & 0.00 \\
\hline 2000 & 0.25 & 0.23 & 0.03 & 0.00 & 0.00 & 0.00 & 0.00 \\
\hline 2000 & 0.25 & 0.28 & 0.05 & 0.00 & 0.00 & 0.00 & 0.00 \\
\hline 2000 & 0.5 & 0.23 & 0.05 & 0.00 & 0.00 & 0.00 & 0.00 \\
\hline 2000 & 0.5 & 0.22 & 0.04 & 0.00 & 0.00 & 0.00 & 0.00 \\
\hline 2000 & 0.5 & 0.24 & 0.03 & 0.00 & 0.00 & 0.00 & 0.00 \\
\hline 2000 & 1 & 0.21 & 0.07 & 0.00 & 0.00 & 0.00 & -0.02 \\
\hline 2000 & 1 & 0.20 & 0.07 & 0.00 & 0.00 & 0.00 & -0.02 \\
\hline 2000 & 1 & 0.22 & 0.08 & 0.00 & 0.00 & 0.00 & -0.02 \\
\hline 2000 & 3 & 0.21 & 0.18 & 0.00 & 0.00 & 0.00 & -0.06 \\
\hline 2000 & 3 & 0.27 & 0.16 & 0.00 & 0.00 & 0.00 & -0.05 \\
\hline 2000 & 3 & 0.21 & 0.18 & 0.00 & 0.00 & 0.00 & -0.05 \\
\hline 2000 & 7 & 0.34 & 0.42 & 0.00 & 0.00 & 0.00 & -0.03 \\
\hline 2000 & 7 & 0.33 & 0.37 & 0.00 & 0.00 & 0.00 & -0.04 \\
\hline 2000 & 7 & 0.34 & 0.39 & 0.00 & 0.00 & 0.00 & -0.03 \\
\hline 2000 & 14 & 0.45 & 0.45 & 0.00 & 0.00 & 0.00 & -0.04 \\
\hline 2000 & 14 & 0.38 & 0.45 & 0.00 & 0.00 & 0.00 & -0.02 \\
\hline 2000 & 14 & 0.40 & 0.47 & 0.00 & 0.00 & 0.00 & -0.06 \\
\hline 2000 & 28 & 0.36 & 0.54 & 0.00 & 0.00 & 0.00 & -0.09 \\
\hline 2000 & 28 & 0.42 & 0.66 & 0.00 & 0.00 & 0.00 & -0.09 \\
\hline 2000 & 28 & 0.39 & 0.68 & 0.00 & 0.00 & 0.00 & -0.10 \\
\hline 2000 & 56 & 0.44 & 0.67 & 0.00 & 0.00 & 0.00 & -0.07 \\
\hline 2000 & 56 & 0.44 & 0.67 & 0.00 & 0.00 & 0.00 & -0.05 \\
\hline 2000 & 56 & 0.45 & 0.68 & 0.00 & 0.00 & 0.00 & -0.05 \\
\hline 2000 & 91 & 0.64 & 0.85 & 0.00 & 0.00 & 0.00 & -0.05 \\
\hline 2000 & 91 & 0.66 & 0.87 & 0.00 & 0.00 & 0.00 & -0.05 \\
\hline 2000 & 91 & 0.67 & 0.82 & 0.00 & 0.00 & 0.00 & -0.04 \\
\hline 2000 & 182 & 0.82 & 0.93 & 0.00 & 0.00 & 0.00 & -0.08 \\
\hline 2000 & 182 & 0.90 & 0.87 & 0.00 & 0.00 & 0.00 & -0.08 \\
\hline 2000 & 182 & 0.89 & 0.89 & 0.00 & 0.00 & 0.00 & -0.09 \\
\hline 20000 & 1 & 0.22 & 0.82 & 0.00 & 0.00 & 0.00 & 0.00 \\
\hline
\end{tabular}


CONC. (ppm, blank corrected) FOR 0.45u FILTERED LEACHATES (all test)

\begin{tabular}{|r|r|r|r|r|r|r|r|}
\hline SA/V & Duration & & & & & & \\
\hline (m-1) & (days) & \multicolumn{1}{c|}{ CS } & CU & DY & EU & FE & \multicolumn{1}{c|}{ GD } \\
\hline 20000 & 1 & 0.23 & 0.76 & 0.00 & 0.00 & 0.00 & 0.00 \\
\hline 20000 & 1 & 0.26 & 0.86 & 0.00 & 0.00 & 0.00 & 0.00 \\
\hline 20000 & 3 & 0.45 & 2.32 & 0.00 & 0.00 & 0.00 & -0.25 \\
\hline 20000 & 3 & 0.44 & 2.27 & 0.00 & 0.00 & 0.00 & -0.21 \\
\hline 20000 & 3 & 0.37 & 2.19 & 0.00 & 0.00 & 0.00 & -0.20 \\
\hline 20000 & 7 & 0.58 & 3.97 & -0.02 & 0.00 & 0.00 & -0.15 \\
\hline 20000 & 7 & 0.73 & 4.13 & -0.01 & 0.01 & 0.01 & -0.15 \\
\hline 20000 & 7 & 0.69 & 4.04 & -0.02 & 0.00 & 0.00 & -0.20 \\
\hline 20000 & 14 & 0.58 & 4.80 & 0.00 & 0.00 & 0.00 & -0.35 \\
\hline 20000 & 14 & 0.53 & 4.90 & 0.00 & 0.00 & 0.00 & -0.36 \\
\hline 20000 & 14 & 0.53 & 4.97 & 0.00 & 0.00 & 0.00 & -0.35 \\
\hline 20000 & 28 & 0.14 & 0.02 & 0.00 & 0.00 & 0.00 & -0.15 \\
\hline 20000 & 28 & 0.17 & 0.01 & 0.01 & 0.00 & 0.00 & -0.15 \\
\hline 20000 & 28 & 0.12 & 0.01 & 0.02 & 0.00 & 0.00 & 0.02 \\
\hline
\end{tabular}


CONC. (ppm, blank corrected) FOR 0.45u FILTERED LEACHATES (all test)

\begin{tabular}{|c|c|c|c|c|c|c|c|}
\hline $\mathrm{SA} / \mathrm{V}$ & Duration & & & & & & \\
\hline$(m-1)$ & (days) & $\mathbf{K}$ & LA & LI & MG & $\mathbf{M N}$ & $\mathrm{MO}$ \\
\hline 10 & 0.25 & 0.01 & 0.00 & 0.05 & 0.00 & 0.00 & 0.01 \\
\hline 10 & 0.25 & 0.00 & 0.00 & 0.07 & 0.01 & 0.00 & 0.01 \\
\hline 10 & 0.25 & 0.00 & 0.00 & 0.06 & 0.00 & 0.00 & 0.01 \\
\hline 10 & 0.5 & 0.00 & 0.00 & 0.21 & 0.03 & 0.00 & 0.00 \\
\hline 10 & 0.5 & 0.00 & 0.00 & 0.28 & 0.04 & 0.00 & 0.03 \\
\hline 10 & 0.5 & 0.00 & 0.00 & 0.30 & 0.05 & 0.00 & 0.03 \\
\hline 10 & 1) & 0.00 & 0.00 & 0.49 & 0.10 & 0.00 & 0.05 \\
\hline 10 & 1 & 0.00 & 0.00 & 0.49 & 0.10 & 0.00 & 0.05 \\
\hline 10 & 1 & 0.00 & 0.00 & 0.48 & 0.09 & 0.00 & $\overline{0.04}$ \\
\hline 10 & 3 & 0.00 & 0.00 & 1.22 & 0.16 & 0.00 & 0.13 \\
\hline 10 & 3 & 0.00 & 0.00 & 1.11 & 0.20 & 0.00 & 0.13 \\
\hline 10 & 3 & 0.00 & 0.00 & 1.11 & 0.20 & 0.00 & 0.12 \\
\hline 10 & 7 & 0.00 & 0.00 & 1.63 & 0.04 & 0.00 & 0.18 \\
\hline 10 & 7 & 0.00 & 0.00 & 1.66 & 0.04 & 0.00 & 0.18 \\
\hline 10 & 7 & 0.00 & 0.00 & 1.60 & 0.03 & 0.00 & 0.17 \\
\hline 10 & 14 & 0.00 & 0.00 & 1.95 & 0.02 & 0.00 & 0.22 \\
\hline 10 & 14 & 0.00 & 0.00 & 1.99 & 0.01 & 0.00 & 0.22 \\
\hline 10 & 14 & 0.00 & 0.00 & 2.02 & 0.01 & 0.00 & 0.22 \\
\hline 10 & 28 & 0.00 & 0.00 & 2.42 & 0.00 & 0.00 & 0.26 \\
\hline 10 & 28 & 0.00 & 0.00 & 2.49 & 0.02 & 0.00 & 0.26 \\
\hline 10 & 28 & 0.00 & 0.00 & 2.48 & 0.02 & 0.00 & 0.26 \\
\hline 10 & 56 & 0.00 & 0.00 & 2.73 & 0.04 & 0.01 & 0.30 \\
\hline 10 & 56 & 0.00 & 0.00 & 2.73 & 0.03 & 0.01 & 0.29 \\
\hline 10 & 56 & 0.00 & 0.00 & 2.76 & 0.02 & 0.00 & 0.30 \\
\hline 10 & 91 & 0.00 & -0.01 & 2.96 & 0.02 & 0.01 & 0.32 \\
\hline 10 & 91 & 0.00 & -0.01 & 2.95 & 0.02 & 0.01 & 0.33 \\
\hline 10 & 91 & 0.00 & -0.01 & 2.94 & 0.00 & 0.01 & 0.32 \\
\hline 10 & 182 & 0.09 & 0.00 & 3.26 & 0.06 & 0.01 & 0.36 \\
\hline 10 & 182 & 0.18 & 0.00 & 3.19 & 0.08 & 0.01 & 0.35 \\
\hline 10 & 182 & -0.07 & 0.00 & 3.38 & 0.05 & 0.01 & 0.37 \\
\hline 10 & 364 & 0.00 & 0.00 & 3.73 & 0.06 & 0.01 & 0.42 \\
\hline 10 & 364 & 0.00 & 0.01 & 3.96 & 0.08 & 0.01 & 0.43 \\
\hline 10 & 364 & 0.00 & 0.01 & 3.96 & 0.08 & 0.01 & 0.43 \\
\hline 530 & 0.25 & 0.00 & 0.05 & 2.11 & 0.18 & 0.02 & 0.19 \\
\hline 530 & 0.25 & 0.00 & 0.08 & 2.21 & 0.19 & 0.02 & 0.20 \\
\hline 530 & 0.25 & 0.00 & 0.09 & 2.36 & 0.18 & 0.02 & 0.21 \\
\hline 530 & 0.5 & 0.00 & 0.14 & 2.83 & 0.20 & 0.04 & 0.26 \\
\hline 530 & 0.5 & 0.00 & 0.12 & 2.89 & 0.17 & 0.03 & 0.26 \\
\hline 530 & 0.5 & 0.00 & 0.08 & 2.91 & 0.16 & 0.02 & 0.26 \\
\hline 530 & 1 & 0.00 & 0.18 & 3.61 & 0.29 & 0.06 & 0.34 \\
\hline 530 & 1 & 0.00 & 0.15 & 3.60 & 0.23 & 0.05 & 0.34 \\
\hline 530 & 1 & 0.00 & 0.16 & 3.48 & 0.25 & 0.06 & 0.33 \\
\hline 530 & 3 & 0.00 & 0.11 & 5.34 & 0.56 & 0.08 & 0.54 \\
\hline 530 & 3 & 0.00 & 0.14 & 5.38 & 0.64 & 0.10 & 0.55 \\
\hline 530 & 3 & 0.00 & 0.17 & 5.36 & 0.71 & 0.11 & 0.55 \\
\hline 530 & 7 & 0.00 & 0.16 & 7.51 & 1.11 & 0.18 & 0.75 \\
\hline 530 & 7 & 0.00 & 0.16 & 7.40 & 1.11 & 0.18 & 0.74 \\
\hline
\end{tabular}


CONC. (ppm, blank corrected) FOR 0.45u FILTERED LEACHATES (all test)

\begin{tabular}{|c|c|c|c|c|c|c|c|}
\hline SA/V & Duration & & & & & & \\
\hline$(m-1)$ & (days) & $\overline{\mathrm{K}}$ & LA & LI & MG & $\overline{\mathrm{MN}}$ & $\mathrm{MO}$ \\
\hline 530 & 7 & 0.00 & 0.14 & 7.20 & 0.94 & 0.15 & 0.71 \\
\hline 530 & 14 & -0.14 & 0.20 & 8.50 & 1.43 & 0.23 & 0.87 \\
\hline 530 & 14 & -0.14 & 0.15 & 8.37 & 1.30 & 0.21 & 0.87 \\
\hline 530 & 14 & -0.14 & 0.19 & 8.40 & 1.39 & 0.23 & 0.86 \\
\hline 530 & 28 & 0.00 & 0.29 & 9.80 & 1.65 & 0.29 & 0.96 \\
\hline 530 & 28 & 0.00 & 0.30 & 9.82 & 1.69 & 0.30 & 0.99 \\
\hline 530 & 28 & 0.00 & 0.26 & 9.76 & 1.61 & 0.28 & 0.98 \\
\hline 530 & 56 & 0.07 & 0.20 & 11.00 & 1.47 & 0.25 & 1.01 \\
\hline 530 & 56 & 0.00 & 0.15 & 10.80 & 0.83 & 0.14 & 1.01 \\
\hline 530 & 56 & 0.00 & 0.30 & 11.20 & 1.68 & 0.29 & 1.03 \\
\hline 530 & 91 & 0.00 & 0.22 & 11.90 & 1.35 & 0.22 & 1.11 \\
\hline 530 & 91 & 0.00 & 0.23 & 12.10 & 1.59 & 0.26 & 1.12 \\
\hline 530 & 91 & 0.00 & 0.22 & 12.10 & 1.56 & 0.26 & 1.12 \\
\hline 530 & 182 & 0.00 & 0.25 & 13.00 & 1.59 & 0.27 & 1.15 \\
\hline 530 & 182 & 0.00 & 0.31 & 13.50 & 1.62 & 0.28 & 1.19 \\
\hline 530 & 182 & 0.00 & 0.29 & 13.70 & 1.59 & 0.28 & 1.19 \\
\hline 2000 & 0.25 & 0.03 & 0.04 & 4.86 & 0.06 & 0.01 & 0.39 \\
\hline 2000 & 0.25 & 0.02 & 0.04 & 4.77 & 0.07 & 0.01 & 0.39 \\
\hline 2000 & 0.25 & 0.02 & 0.07 & 4.77 & 0.08 & 0.02 & 0.38 \\
\hline 2000 & 0.5 & 0.03 & 0.07 & 6.02 & 0.07 & 0.02 & 0.50 \\
\hline 2000 & 0.5 & 0.00 & 0.06 & 5.90 & 0.06 & 0.02 & 0.50 \\
\hline 2000 & 0.5 & 0.00 & 0.06 & 5.02 & 0.07 & 0.02 & 0.43 \\
\hline 2000 & 1 & 0.00 & 0.09 & 8.21 & 0.22 & 0.04 & 0.75 \\
\hline 2000 & 1 & 0.00 & 0.10 & 8.47 & 0.26 & 0.04 & 0.78 \\
\hline 2000 & 1 & 0.00 & 0.11 & 8.73 & 0.31 & 0.05 & 0.80 \\
\hline 2000 & 3 & 0.00 & 0.20 & 12.89 & 1.63 & 0.21 & 1.20 \\
\hline 2000 & 3 & 0.00 & 0.16 & 12.79 & 1.52 & 0.19 & 1.20 \\
\hline 2000 & 3 & 0.00 & 0.19 & 12.79 & 1.56 & 0.20 & 1.19 \\
\hline 2000 & 7 & 0.00 & 0.31 & 16.30 & 2.66 & 0.42 & 1.44 \\
\hline 2000 & 7 & 0.00 & 0.27 & 16.10 & 2.48 & 0.38 & $1 . \overline{44}$ \\
\hline 2000 & 7 & 0.00 & $0 . \overline{25}$ & 16.10 & 2.57 & 0.40 & $1 . \overline{44}$ \\
\hline 2000 & 14 & 0.00 & 0.31 & 18.30 & 3.05 & 0.48 & 1.67 \\
\hline 2000 & 14 & 0.00 & 0.29 & 18.10 & 2.96 & 0.46 & 1.66 \\
\hline 2000 & 14 & 0.00 & 0.34 & 18.00 & 3.05 & 0.49 & 1.62 \\
\hline 2000 & 28 & 0.00 & 0.30 & 20.30 & 3.21 & 0.52 & 1.75 \\
\hline 2000 & 28 & 0.00 & 0.39 & 22.20 & 3.55 & 0.59 & 1.85 \\
\hline 2000 & 28 & 0.00 & 0.44 & 21.90 & 3.53 & 0.59 & 1.83 \\
\hline 2000 & 56 & 0.00 & 0.52 & 23.90 & $3 . \overline{37}$ & 0.58 & 1.91 \\
\hline 2000 & 56 & 0.00 & 0.50 & 24.10 & 3.40 & 0.58 & 1.93 \\
\hline 2000 & 56 & 0.00 & 0.54 & 23.20 & 3.31 & 0.57 & 1.87 \\
\hline 2000 & 91 & 0.00 & 0.71 & 26.70 & 4.25 & 0.72 & 2.07 \\
\hline 2000 & 91 & 0.00 & 0.74 & 27.30 & 4.33 & 0.73 & 2.10 \\
\hline 2000 & 91 & 0.00 & 0.68 & 25.90 & 4.17 & 0.70 & 2.02 \\
\hline 2000 & 182 & 0.00 & 1.08 & 30.70 & 4.43 & 0.78 & 2.26 \\
\hline 2000 & 182 & 0.00 & 1.17 & 31.60 & 4.16 & 0.72 & 2.26 \\
\hline 2000 & 182 & 0.00 & 1.14 & 31.10 & 4.13 & 0.71 & 2.21 \\
\hline 20000 & 1 & 0.00 & 0.22 & 42.65 & 4.07 & 0.53 & 3.52 \\
\hline
\end{tabular}


CONC. (ppm, blank corrected) FOR 0.45u FILTERED LEACHATES (all test)

\begin{tabular}{|r|r|r|r|r|r|r|r|}
\hline SA/V & Duration & & & & & & \\
\hline$(\mathrm{m}-1)$ & (days) & \multicolumn{1}{|c|}{ K } & LA & LI & MG & MN & \multicolumn{1}{c|}{ MO } \\
\hline 20000 & 1 & 0.06 & 0.20 & 42.98 & 3.69 & 0.45 & 3.47 \\
\hline 20000 & 1 & 0.00 & 0.23 & 48.21 & 3.96 & 0.47 & 3.83 \\
\hline 20000 & 3 & 0.00 & 0.46 & 67.45 & 10.24 & 1.70 & 5.75 \\
\hline 20000 & 3 & 0.00 & 0.39 & 64.70 & 8.97 & 1.50 & 5.28 \\
\hline 20000 & 3 & 0.00 & 0.43 & 63.45 & 8.69 & 1.46 & 5.25 \\
\hline 20000 & 7 & -0.41 & 0.68 & 79.43 & 12.95 & 2.28 & 6.28 \\
\hline 20000 & 7 & -0.41 & 0.74 & 78.93 & 13.20 & 2.31 & 6.18 \\
\hline 20000 & 7 & -0.41 & 0.70 & 77.90 & 12.93 & 2.28 & 6.14 \\
\hline 20000 & 14 & 0.00 & 0.98 & 94.00 & 14.00 & 2.62 & 6.95 \\
\hline 20000 & 14 & 0.00 & 1.04 & 91.62 & 14.35 & 2.68 & 6.72 \\
\hline 20000 & 14 & 0.00 & 1.26 & 91.50 & 14.90 & 2.79 & 6.65 \\
\hline 20000 & 28 & 0.00 & 0.09 & 89.37 & 0.07 & 0.01 & 8.30 \\
\hline 20000 & 28 & 0.00 & 0.10 & 89.37 & 0.06 & 0.01 & 8.25 \\
\hline 20000 & 28 & 0.00 & 0.13 & 85.67 & 0.10 & 0.00 & 8.01 \\
\hline
\end{tabular}


CONC. (ppm, blank corrected) FOR 0.45u FILTERED LEACHATES (all test)

\begin{tabular}{|c|c|c|c|c|c|c|c|}
\hline SA/V & Duration & & & & & & \\
\hline$(m-1)$ & (days) & NA & ND & NI & $\mathbf{P}$ & PB & RU \\
\hline 10 & 0.25 & 0.24 & 0.00 & 0.00 & -0.01 & 0.00 & 0.00 \\
\hline 10 & 0.25 & 0.27 & 0.00 & 0.00 & -0.01 & 0.00 & 0.00 \\
\hline 10 & 0.25 & 0.23 & 0.00 & 0.00 & -0.01 & 0.00 & 0.00 \\
\hline 10 & 0.5 & 0.89 & 0.00 & 0.00 & 0.00 & 0.00 & 0.00 \\
\hline 10 & 0.5 & 1.18 & 0.00 & 0.00 & 0.00 & 0.00 & 0.00 \\
\hline 10 & 0.5 & 1.24 & 0.00 & 0.00 & 0.00 & 0.00 & 0.00 \\
\hline 10 & 1 & 2.13 & 0.00 & 0.00 & 0.00 & 0.00 & 0.00 \\
\hline 10 & 1 & 2.17 & 0.00 & 0.00 & 0.00 & 0.00 & 0.00 \\
\hline 10 & 1 & 1.90 & 0.00 & 0.00 & 0.00 & 0.00 & 0.00 \\
\hline 10 & 3 & 5.10 & 0.00 & 0.00 & 0.00 & 0.00 & 0.00 \\
\hline 10 & 3 & 4.74 & 0.00 & 0.01 & 0.00 & 0.00 & 0.00 \\
\hline 10 & 3 & 4.78 & 0.00 & 0.00 & -0.02 & 0.00 & 0.00 \\
\hline 10 & 7 & 7.02 & 0.00 & 0.00 & -0.01 & -0.02 & 0.00 \\
\hline 10 & 7 & 7.20 & 0.00 & 0.00 & 0.01 & -0.01 & 0.00 \\
\hline 10 & 7 & 6.97 & 0.00 & 0.00 & -0.01 & -0.02 & 0.00 \\
\hline 10 & 14 & 8.67 & 0.00 & 0.01 & 0.03 & -0.02 & 0.00 \\
\hline 10 & 14 & 8.70 & 0.00 & 0.00 & 0.02 & -0.02 & 0.00 \\
\hline 10 & 14 & 8.73 & 0.00 & 0.00 & 0.01 & -0.03 & 0.00 \\
\hline 10 & 28 & 9.88 & 0.00 & 0.00 & -0.01 & -0.03 & 0.00 \\
\hline 10 & 28 & 10.19 & 0.00 & 0.00 & 0.00 & -0.03 & 0.00 \\
\hline 10 & 28 & 10.09 & 0.00 & 0.00 & -0.01 & -0.03 & 0.00 \\
\hline 10 & 56 & 11.30 & 0.00 & 0.01 & 0.01 & -0.02 & 0.00 \\
\hline 10 & 56 & 11.20 & 0.00 & 0.00 & 0.03 & -0.02 & 0.00 \\
\hline 10 & 56 & 11.40 & 0.00 & 0.01 & 0.00 & -0.03 & 0.00 \\
\hline 10 & 91 & 12.70 & 0.00 & 0.00 & 0.02 & -0.04 & 0.00 \\
\hline 10 & 91 & 12.80 & 0.00 & 0.01 & 0.00 & -0.04 & 0.00 \\
\hline 10 & 91 & 12.60 & 0.00 & 0.00 & 0.00 & -0.04 & 0.00 \\
\hline 10 & 182 & 14.35 & 0.00 & 0.00 & 0.07 & -0.01 & 0.00 \\
\hline 10 & 182 & 13.95 & 0.00 & 0.00 & 0.05 & 0.00 & 0.00 \\
\hline 10 & 182 & 14.75 & 0.00 & 0.00 & 0.04 & -0.01 & -0.01 \\
\hline 10 & 364 & 15.25 & 0.01 & 0.02 & 0.07 & 0.02 & 0.00 \\
\hline 10 & 364 & 16.05 & 0.01 & 0.01 & 0.08 & 0.02 & 0.00 \\
\hline 10 & 364 & 16.05 & 0.01 & 0.01 & 0.08 & 0.02 & 0.00 \\
\hline 530 & 0.25 & 8.43 & 0.01 & 0.04 & 0.01 & 0.01 & 0.01 \\
\hline 530 & 0.25 & 8.81 & 0.03 & 0.06 & 0.01 & 0.01 & 0.00 \\
\hline 530 & 0.25 & 9.45 & 0.05 & 0.07 & 0.00 & 0.02 & 0.00 \\
\hline 530 & 0.5 & 11.07 & 0.08 & 0.10 & 0.00 & 0.04 & 0.02 \\
\hline 530 & 0.5 & 11.32 & 0.07 & 0.08 & 0.01 & 0.02 & 0.00 \\
\hline 530 & 0.5 & 11.39 & 0.03 & 0.06 & 0.00 & -0.01 & 0.00 \\
\hline 530 & 1 & 14.90 & 0.11 & 0.18 & 0.03 & -0.04 & 0.00 \\
\hline 530 & 1 & 14.90 & 0.09 & 0.16 & 0.03 & -0.04 & 0.00 \\
\hline 530 & 1 & 14.60 & 0.09 & 0.16 & 0.02 & -0.04 & 0.00 \\
\hline 530 & 3 & 22.48 & 0.07 & 0.20 & 0.05 & -0.03 & 0.00 \\
\hline 530 & 3 & 22.78 & 0.11 & 0.24 & 0.05 & -0.03 & 0.00 \\
\hline 530 & 3 & 22.48 & 0.13 & 0.27 & 0.04 & -0.04 & 0.00 \\
\hline 530 & 7 & 31.50 & 0.12 & 0.38 & 0.01 & -0.05 & 0.00 \\
\hline 530 & 7 & 31.20 & 0.12 & 0.40 & 0.01 & -0.05 & 0.00 \\
\hline
\end{tabular}


CONC. (ppm, blank corrected) FOR 0.45u FILTERED LEACHATES (all test)

\begin{tabular}{|c|c|c|c|c|c|c|c|}
\hline $\mathrm{SA} / \mathrm{V}$ & Duration & & & & & & \\
\hline$(m-1)$ & (days) & NA & ND & NI & $\mathbf{P}$ & PB & RU \\
\hline 530 & 7 & 30.30 & 0.10 & 0.36 & 0.04 & -0.05 & 0.00 \\
\hline 530 & 14 & 35.66 & 0.16 & 0.54 & 0.07 & -0.06 & 1.49 \\
\hline 530 & 14 & 35.16 & 0.12 & 0.44 & 0.08 & -0.05 & 1.35 \\
\hline 530 & 14 & 35.36 & 0.14 & 0.51 & 0.08 & -0.04 & 1.45 \\
\hline 530 & 28 & 39.70 & 0.24 & 0.69 & 0.06 & -0.01 & 0.05 \\
\hline 530 & 28 & 39.90 & 0.24 & 0.67 & 0.05 & -0.01 & 0.07 \\
\hline 530 & 28 & 39.40 & 0.20 & 0.61 & 0.04 & -0.01 & 0.06 \\
\hline 530 & 56 & 42.84 & 0.17 & 0.62 & 0.06 & -0.06 & 0.00 \\
\hline 530 & 56 & 42.14 & 0.12 & 0.38 & 0.08 & -0.06 & 0.00 \\
\hline 530 & 56 & 43.54 & 0.25 & 0.77 & 0.08 & -0.07 & 0.00 \\
\hline 530 & 91 & 47.20 & 0.19 & 0.60 & 0.10 & -0.05 & 0.00 \\
\hline 530 & 91 & 47.90 & 0.20 & 0.71 & 0.09 & -0.06 & 0.00 \\
\hline 530 & 91 & 47.80 & 0.18 & 0.69 & 0.09 & -0.07 & 0.00 \\
\hline 530 & 182 & 49.10 & 0.19 & 0.74 & 0.10 & 0.00 & 0.06 \\
\hline 530 & 182 & 51.00 & 0.26 & 0.82 & 0.08 & 0.00 & 0.06 \\
\hline 530 & 182 & 51.60 & 0.22 & 0.77 & 0.06 & -0.02 & 0.05 \\
\hline 2000 & 0.25 & 18.21 & 0.00 & 0.03 & 0.01 & 0.02 & 0.00 \\
\hline 2000 & 0.25 & 18.00 & 0.01 & 0.03 & 0.02 & 0.00 & 0.00 \\
\hline 2000 & 0.25 & 18.08 & 0.03 & 0.05 & 0.05 & 0.02 & 0.01 \\
\hline 2000 & 0.5 & 22.73 & 0.02 & 0.05 & -0.01 & 0.04 & 0.00 \\
\hline 2000 & 0.5 & 22.65 & 0.02 & 0.05 & -0.01 & 0.02 & 0.00 \\
\hline 2000 & 0.5 & 19.59 & 0.02 & 0.04 & 0.01 & 0.04 & 0.00 \\
\hline 2000 & 1 & 31.30 & 0.07 & 0.14 & 0.06 & -0.05 & 0.01 \\
\hline 2000 & 1 & 32.30 & 0.08 & 0.15 & 0.05 & -0.05 & 0.00 \\
\hline 2000 & 1 & 33.10 & 0.09 & 0.18 & 0.05 & -0.04 & 0.01 \\
\hline 2000 & 3 & 50.50 & 0.14 & 0.54 & 0.04 & -0.11 & 0.00 \\
\hline 2000 & 3 & 50.50 & 0.10 & 0.48 & 0.06 & -0.11 & 0.00 \\
\hline 2000 & 3 & 50.20 & 0.12 & 0.52 & 0.05 & -0.10 & 0.00 \\
\hline 2000 & 7 & 62.30 & 0.26 & 1.13 & 0.09 & -0.08 & 0.00 \\
\hline 2000 & 7 & 61.90 & 0.21 & 1.01 & 0.07 & -0.08 & 0.00 \\
\hline 2000 & 7 & 61.60 & 0.20 & 1.07 & 0.09 & -0.07 & 0.00 \\
\hline 2000 & 14 & 70.38 & 0.26 & 1.29 & 0.10 & -0.08 & 2.88 \\
\hline 2000 & 14 & 69.38 & 0.25 & 1.25 & 0.10 & -0.07 & 2.80 \\
\hline 2000 & 14 & 69.38 & 0.27 & 1.32 & 0.08 & -0.10 & 2.82 \\
\hline 2000 & 28 & 75.20 & 0.24 & 1.31 & 0.08 & -0.01 & 0.07 \\
\hline 2000 & 28 & 79.60 & 0.32 & 1.48 & 0.02 & -0.01 & 0.09 \\
\hline 2000 & 28 & 78.20 & 0.37 & 1.50 & 0.01 & -0.02 & 0.09 \\
\hline 2000 & 56 & 84.10 & 0.47 & 1.75 & 0.15 & -0.09 & -0.01 \\
\hline 2000 & 56 & 84.40 & 0.45 & 1.74 & 0.15 & -0.08 & 0.00 \\
\hline 2000 & 56 & 81.70 & 0.49 & 1.76 & 0.13 & -0.08 & 0.01 \\
\hline 2000 & 91 & 95.80 & 0.65 & 2.22 & 0.13 & -0.09 & 0.00 \\
\hline 2000 & 91 & 97.90 & 0.69 & 2.33 & 0.15 & -0.09 & 0.00 \\
\hline 2000 & 91 & 93.20 & 0.63 & 2.25 & 0.16 & -0.07 & 0.00 \\
\hline 2000 & 182 & 103.00 & 1.00 & 2.53 & 0.15 & 0.01 & 0.21 \\
\hline 2000 & 182 & 103.00 & 1.08 & 2.40 & 0.17 & 0.00 & 0.17 \\
\hline 2000 & 182 & 101.00 & 1.06 & 2.38 & 0.16 & 0.01 & 0.17 \\
\hline 20000 & 1 & 142.43 & 0.09 & 1.51 & 0.18 & 0.00 & 0.06 \\
\hline
\end{tabular}


CONC. (ppm, blank corrected) FOR 0.45u FILTERED LEACHATES (all test)

\begin{tabular}{|r|r|r|r|r|r|r|r|}
\hline SA/V & Duration & & & & & & \\
\hline (m-1) & (days) & NA & ND & NI & \multicolumn{1}{|c|}{ P } & \multicolumn{1}{|c|}{ PB } & \multicolumn{1}{|c|}{ RU } \\
\hline 20000 & 1 & 142.17 & 0.11 & 1.31 & 0.18 & 0.14 & 0.04 \\
\hline 20000 & 1 & 158.84 & 0.05 & 1.38 & 0.19 & 0.06 & 0.03 \\
\hline 20000 & 3 & 231.89 & 0.32 & 4.57 & 0.24 & -0.29 & 0.00 \\
\hline 20000 & 3 & 220.55 & 0.14 & 4.19 & 0.22 & -0.34 & 0.00 \\
\hline 20000 & 3 & 217.39 & 0.22 & 4.05 & 0.19 & -0.34 & 0.00 \\
\hline 20000 & 7 & 265.20 & 0.61 & 6.45 & 0.33 & -0.37 & 0.00 \\
\hline 20000 & 7 & 262.70 & 0.65 & 6.60 & 0.23 & -0.32 & 0.00 \\
\hline 20000 & 7 & 260.30 & 0.63 & 6.51 & 0.26 & -0.32 & 0.00 \\
\hline 20000 & 14 & 302.50 & 0.83 & 7.20 & 0.26 & -0.50 & 0.67 \\
\hline 20000 & 14 & 293.18 & 0.86 & 7.38 & 0.32 & -0.46 & 0.60 \\
\hline 20000 & 14 & 291.50 & 1.08 & 7.85 & 0.28 & -0.45 & 0.66 \\
\hline 20000 & 28 & 314.62 & 0.00 & 0.01 & 0.31 & 0.00 & 0.00 \\
\hline 20000 & 28 & 316.62 & 0.00 & 0.00 & 0.28 & 0.00 & 0.00 \\
\hline 20000 & 28 & 306.52 & 0.00 & 0.00 & 0.18 & 0.00 & 0.03 \\
\hline
\end{tabular}


CONC. (ppm, blank corrected) FOR 0.45u FILTERED LEACHATES (all test)

\begin{tabular}{|c|c|c|c|c|c|c|c|}
\hline $\mathrm{SA} / \mathrm{V}$ & Duration & & & & & & \\
\hline$(m-1)$ & (days) & SB & SI & SR & $\mathrm{TE}$ & TI & $\mathrm{V}$ \\
\hline 10 & 0.25 & 0.00 & 0.62 & 0.00 & 0.00 & 0.00 & 0.00 \\
\hline 10 & 0.25 & 0.00 & 0.79 & 0.00 & 0.00 & 0.00 & 0.00 \\
\hline 10 & 0.25 & 0.00 & 0.84 & 0.00 & 0.01 & 0.00 & 0.00 \\
\hline 10 & 0.5 & 0.00 & 2.91 & 0.01 & 0.00 & 0.00 & 0.00 \\
\hline 10 & 0.5 & 0.00 & 3.79 & 0.01 & 0.00 & 0.00 & 0.00 \\
\hline 10 & 0.5 & 0.00 & 4.01 & 0.01 & 0.00 & 0.00 & 0.00 \\
\hline 10 & 1 & 0.00 & 6.57 & 0.01 & 0.00 & 0.00 & 0.00 \\
\hline 10 & 1 & 0.00 & 6.85 & 0.02 & 0.00 & 0.00 & 0.00 \\
\hline 10 & 1 & 0.00 & 6.26 & 0.01 & 0.00 & 0.00 & 0.00 \\
\hline 10 & 3 & 0.01 & 15.17 & 0.03 & 0.00 & 0.00 & 0.00 \\
\hline 10 & 3 & 0.01 & 14.17 & 0.03 & 0.00 & 0.00 & 0.00 \\
\hline 10 & 3 & 0.00 & 13.97 & 0.03 & 0.00 & 0.00 & 0.00 \\
\hline 10 & 7 & 0.01 & 19.47 & 0.04 & -0.01 & 0.00 & 0.00 \\
\hline 10 & 7 & 0.01 & 19.77 & 0.04 & 0.00 & 0.00 & 0.00 \\
\hline 10 & 7 & 0.01 & 19.07 & 0.04 & -0.01 & 0.00 & 0.00 \\
\hline 10 & 14 & 0.00 & 23.19 & 0.04 & -0.01 & 0.00 & 0.00 \\
\hline 10 & 14 & 0.00 & 23.29 & 0.04 & -0.01 & 0.00 & 0.00 \\
\hline 10 & 14 & 0.00 & 23.39 & 0.04 & -0.02 & 0.00 & 0.00 \\
\hline 10 & 28 & 0.01 & 26.59 & 0.04 & -0.01 & 0.00 & 0.00 \\
\hline 10 & 28 & 0.00 & 27.09 & 0.04 & -0.01 & 0.00 & 0.00 \\
\hline 10 & 28 & 0.00 & 26.99 & 0.04 & -0.01 & 0.00 & 0.00 \\
\hline 10 & 56 & 0.01 & 29.78 & 0.04 & 0.01 & 0.00 & 0.00 \\
\hline 10 & 56 & 0.01 & 29.28 & 0.04 & 0.01 & 0.00 & 0.00 \\
\hline 10 & 56 & 0.00 & 29.68 & 0.04 & 0.00 & 0.00 & 0.00 \\
\hline 10 & 91 & 0.00 & 31.96 & 0.04 & -0.03 & 0.00 & 0.00 \\
\hline 10 & 91 & 0.00 & 32.16 & 0.04 & -0.03 & 0.00 & 0.00 \\
\hline 10 & 91 & 0.00 & 31.96 & 0.04 & -0.02 & 0.00 & 0.00 \\
\hline 10 & 182 & 0.00 & 35.20 & 0.04 & -0.02 & 0.00 & 0.00 \\
\hline 10 & 182 & 0.00 & 34.50 & 0.04 & -0.01 & 0.00 & 0.00 \\
\hline 10 & 182 & 0.00 & 36.60 & 0.04 & -0.02 & 0.00 & 0.00 \\
\hline 10 & 364 & 0.03 & 39.07 & 0.04 & -0.01 & 0.00 & 0.00 \\
\hline 10 & 364 & 0.00 & 40.97 & 0.04 & -0.02 & 0.00 & 0.00 \\
\hline 10 & 364 & 0.00 & 40.97 & 0.04 & -0.02 & 0.00 & 0.00 \\
\hline 530 & 0.25 & 0.02 & 22.38 & 0.03 & 0.10 & 0.00 & 0.00 \\
\hline 530 & 0.25 & 0.02 & 23.59 & 0.03 & 0.16 & 0.00 & 0.00 \\
\hline 530 & 0.25 & 0.01 & 24.98 & 0.03 & 0.06 & 0.00 & 0.00 \\
\hline 530 & 0.5 & -0.01 & 28.46 & 0.03 & -0.03 & 0.00 & 0.00 \\
\hline 530 & 0.5 & 0.01 & 28.85 & 0.02 & 0.03 & 0.00 & 0.00 \\
\hline 530 & 0.5 & 0.01 & 28.49 & 0.02 & -0.10 & 0.00 & 0.00 \\
\hline 530 & 1 & 0.00 & 33.99 & 0.03 & -0.02 & 0.00 & 0.00 \\
\hline 530 & 1 & -0.01 & 33.49 & 0.02 & -0.02 & 0.00 & 0.00 \\
\hline 530 & 1 & 0.00 & 32.99 & 0.02 & -0.02 & 0.00 & 0.00 \\
\hline 530 & 3 & 0.01 & 48.19 & 0.02 & -0.01 & 0.00 & 0.00 \\
\hline 530 & 3 & 0.02 & 49.49 & 0.03 & -0.01 & 0.01 & 0.00 \\
\hline 530 & 3 & 0.02 & 49.29 & 0.03 & -0.03 & 0.01 & 0.00 \\
\hline 530 & 7 & 0.02 & 62.80 & 0.04 & 0.01 & 0.01 & 0.00 \\
\hline 530 & 7 & 0.02 & 63.10 & 0.04 & -0.02 & 0.01 & 0.00 \\
\hline
\end{tabular}


CONC. (ppm, blank corrected) FOR 0.45u FILTERED LEACHATES (all test)

\begin{tabular}{|c|c|c|c|c|c|c|c|}
\hline $\mathrm{SA} / \mathrm{V}$ & Duration & & & & & & \\
\hline$(m-1)$ & (days) & SB & SI & SR & TE & TI & V \\
\hline 530 & 7 & 0.02 & 60.20 & 0.04 & -0.01 & 0.01 & 0.00 \\
\hline 530 & 14 & 0.01 & 73.30 & 0.05 & -0.01 & 0.01 & 0.00 \\
\hline 530 & 14 & 0.01 & 72.00 & 0.05 & -0.02 & 0.01 & 0.00 \\
\hline 530 & 14 & 0.01 & 72.50 & 0.05 & -0.03 & 0.01 & 0.00 \\
\hline 530 & 28 & 0.05 & 80.44 & 0.07 & -0.02 & 0.01 & 0.00 \\
\hline 530 & 28 & 0.05 & 100.94 & 0.07 & -0.03 & 0.02 & 0.00 \\
\hline 530 & 28 & 0.05 & 99.04 & 0.07 & -0.03 & 0.01 & 0.00 \\
\hline 530 & 56 & 0.04 & 80.37 & 0.06 & 0.03 & 0.01 & 0.01 \\
\hline 530 & 56 & 0.01 & 75.97 & 0.04 & 0.03 & 0.01 & 0.00 \\
\hline 530 & 56 & 0.04 & 82.27 & 0.07 & 0.04 & 0.01 & 0.01 \\
\hline 530 & 91 & 0.02 & 85.39 & 0.06 & 0.02 & 0.01 & 0.01 \\
\hline 530 & 91 & 0.02 & 87.89 & 0.07 & 0.00 & 0.01 & 0.01 \\
\hline 530 & 91 & 0.02 & 87.69 & 0.07 & 0.02 & 0.01 & 0.01 \\
\hline 530 & 182 & 0.04 & 94.40 & 0.07 & 0.01 & 0.01 & 0.00 \\
\hline 530 & 182 & 0.03 & 95.30 & 0.08 & -0.01 & 0.01 & 0.00 \\
\hline 530 & 182 & 0.02 & 95.20 & 0.08 & -0.01 & 0.01 & 0.00 \\
\hline 2000 & 0.25 & -0.04 & 37.49 & 0.01 & -0.03 & 0.00 & 0.00 \\
\hline 2000 & 0.25 & -0.02 & 37.40 & 0.01 & -0.06 & 0.00 & 0.00 \\
\hline 2000 & 0.25 & -0.03 & 38.19 & 0.01 & -0.06 & 0.00 & 0.00 \\
\hline 2000 & 0.5 & 0.01 & 45.98 & 0.01 & 0.29 & 0.00 & 0.00 \\
\hline 2000 & 0.5 & 0.01 & 45.89 & 0.01 & 0.10 & 0.00 & 0.00 \\
\hline 2000 & 0.5 & -0.01 & 41.33 & 0.01 & 0.16 & 0.00 & 0.00 \\
\hline 2000 & 1 & 0.01 & 57.60 & 0.01 & 0.04 & 0.00 & 0.00 \\
\hline 2000 & 1 & 0.01 & 58.90 & 0.01 & 0.03 & 0.00 & 0.00 \\
\hline 2000 & 1 & 0.01 & 60.90 & 0.01 & 0.05 & 0.00 & 0.00 \\
\hline 2000 & 3 & 0.00 & 83.40 & 0.03 & 0.00 & 0.01 & 0.00 \\
\hline 2000 & 3 & 0.02 & 83.50 & 0.02 & 0.01 & 0.01 & 0.00 \\
\hline 2000 & 3 & 0.01 & 83.00 & 0.02 & 0.00 & 0.01 & 0.00 \\
\hline 2000 & 7 & 0.03 & 98.70 & 0.04 & 0.01 & 0.02 & 0.01 \\
\hline 2000 & 7 & 0.03 & 97.50 & 0.04 & 0.03 & 0.02 & 0.01 \\
\hline 2000 & 7 & 0.01 & 98.10 & 0.04 & 0.03 & 0.02 & 0.01 \\
\hline 2000 & 14 & 0.01 & 109.00 & 0.05 & 0.01 & 0.02 & 0.01 \\
\hline 2000 & 14 & 0.06 & 107.00 & 0.05 & 0.02 & 0.02 & 0.01 \\
\hline 2000 & 14 & 0.01 & 106.00 & 0.05 & -0.02 & 0.02 & 0.01 \\
\hline 2000 & 28 & 0.06 & 113.94 & 0.06 & 0.06 & 0.02 & 0.00 \\
\hline 2000 & 28 & 0.06 & 116.94 & 0.06 & 0.04 & 0.02 & 0.00 \\
\hline 2000 & 28 & 0.06 & 116.94 & 0.07 & 0.03 & 0.02 & 0.00 \\
\hline 2000 & 56 & 0.06 & 112.99 & 0.08 & 0.09 & 0.03 & 0.01 \\
\hline 2000 & 56 & 0.06 & 113.99 & 0.07 & 0.09 & 0.03 & 0.01 \\
\hline 2000 & 56 & 0.06 & 112.99 & 0.08 & 0.09 & 0.03 & 0.01 \\
\hline 2000 & 91 & 0.06 & 122.00 & 0.11 & -0.01 & 0.03 & 0.01 \\
\hline 2000 & 91 & 0.07 & 123.00 & 0.11 & 0.00 & 0.03 & 0.02 \\
\hline 2000 & 91 & 0.07 & 122.00 & 0.11 & 0.03 & 0.03 & 0.02 \\
\hline 2000 & 182 & 0.05 & 149.00 & 0.14 & 0.02 & 0.03 & 0.01 \\
\hline 2000 & 182 & 0.05 & 133.00 & 0.15 & 0.03 & 0.02 & 0.01 \\
\hline 2000 & 182 & 0.05 & 133.00 & 0.15 & 0.05 & 0.02 & 0.01 \\
\hline 20000 & 1 & 0.10 & 188.61 & 0.02 & 0.64 & 0.01 & 0.00 \\
\hline
\end{tabular}


CONC. (ppm, blank corrected) FOR 0.45u FILTERED LEACHATES (all test)

\begin{tabular}{|r|r|r|r|r|r|r|r|}
\hline SA/V & Duration & & & & & & \\
\hline (m-1) & (days) & \multicolumn{1}{|c|}{ SB } & SI & \multicolumn{1}{|c|}{ SR } & \multicolumn{1}{l|}{ TE } & \multicolumn{1}{c|}{ TI } & V \\
\hline 20000 & 1 & 0.21 & 186.96 & 0.02 & 0.76 & 0.00 & 0.00 \\
\hline 20000 & 1 & 0.06 & 213.06 & 0.02 & 0.72 & 0.00 & 0.00 \\
\hline 20000 & 3 & 0.06 & 277.42 & 0.04 & 0.30 & 0.07 & 0.01 \\
\hline 20000 & 3 & -0.01 & 259.78 & 0.04 & 0.22 & 0.05 & 0.00 \\
\hline 20000 & 3 & -0.01 & 268.42 & 0.04 & 0.23 & 0.06 & 0.00 \\
\hline 20000 & 7 & 0.06 & 332.24 & 0.06 & 0.70 & 0.12 & 0.03 \\
\hline 20000 & 7 & 0.12 & 327.74 & 0.06 & 0.65 & 0.12 & 0.03 \\
\hline 20000 & 7 & 0.10 & 310.10 & 0.06 & 0.63 & 0.12 & 0.03 \\
\hline 20000 & 14 & 0.06 & 385.47 & 0.07 & 0.65 & 0.18 & 0.01 \\
\hline 20000 & 14 & 0.05 & 374.08 & 0.07 & 0.64 & 0.17 & 0.01 \\
\hline 20000 & 14 & 0.08 & 366.47 & 0.09 & 0.58 & 0.19 & 0.01 \\
\hline 20000 & 28 & 0.08 & 289.44 & 0.01 & 0.28 & 0.00 & 0.01 \\
\hline 20000 & 28 & 0.08 & 289.44 & 0.01 & 0.17 & 0.00 & 0.01 \\
\hline 20000 & 28 & 0.11 & 285.94 & 0.01 & 0.19 & 0.01 & 0.01 \\
\hline
\end{tabular}


CONC. (ppm, blank corrected) FOR 0.45u FILTERED LEACHATES (all test)

\begin{tabular}{|c|c|c|c|c|}
\hline $\mathrm{SA} / \mathrm{V}$ & Duration & & & \\
\hline$(m-1)$ & (days) & $\mathbf{Y}$ & $\mathrm{ZN}$ & ZR \\
\hline 10 & 0.25 & 0.00 & -0.02 & 0.00 \\
\hline 10 & 0.25 & 0.00 & -0.02 & 0.00 \\
\hline 10 & 0.25 & 0.00 & -0.02 & 0.00 \\
\hline 10 & 0.5 & 0.00 & 0.00 & 0.00 \\
\hline 10 & 0.5 & 0.00 & 0.00 & 0.00 \\
\hline 10 & 0.5 & 0.00 & 0.00 & 0.00 \\
\hline 10 & 1 & 0.00 & 0.00 & 0.00 \\
\hline 10 & 1 & 0.00 & 0.00 & 0.00 \\
\hline 10 & 1 & 0.00 & 0.00 & 0.00 \\
\hline 10 & 3 & 0.00 & 0.00 & 0.00 \\
\hline 10 & 3 & 0.00 & 0.00 & 0.00 \\
\hline 10 & 3 & 0.00 & 0.00 & 0.00 \\
\hline 10 & 7 & 0.00 & 0.00 & 0.00 \\
\hline 10 & 7 & 0.00 & 0.00 & 0.00 \\
\hline 10 & 7 & 0.00 & 0.00 & 0.00 \\
\hline 10 & 14 & 0.00 & 0.00 & 0.00 \\
\hline 10 & 14 & 0.00 & 0.00 & 0.00 \\
\hline 10 & 14 & 0.00 & 0.00 & 0.00 \\
\hline 10 & 28 & 0.00 & 0.00 & -0.01 \\
\hline 10 & 28 & 0.00 & 0.00 & -0.01 \\
\hline 10 & 28 & 0.00 & 0.00 & -0.01 \\
\hline 10 & 56 & 0.00 & -0.04 & 0.00 \\
\hline 10 & 56 & 0.00 & -0.01 & 0.00 \\
\hline 10 & 56 & 0.00 & -0.04 & 0.00 \\
\hline 10 & 91 & 0.00 & 0.00 & 0.01 \\
\hline 10 & 91 & 0.00 & 0.00 & 0.01 \\
\hline 10 & 91 & 0.00 & 0.00 & 0.01 \\
\hline 10 & 182 & 0.00 & 0.00 & 0.00 \\
\hline 10 & 182 & 0.00 & 0.00 & 0.00 \\
\hline 10 & 182 & 0.00 & 0.00 & 0.00 \\
\hline 10 & 364 & 0.00 & 0.00 & 0.00 \\
\hline 10 & 364 & 0.00 & 0.00 & 0.00 \\
\hline 10 & 364 & 0.00 & 0.00 & 0.00 \\
\hline 530 & 0.25 & 0.00 & 0.00 & 0.03 \\
\hline 530 & 0.25 & 0.00 & 0.00 & 0.05 \\
\hline 530 & 0.25 & 0.00 & 0.00 & 0.05 \\
\hline 530 & 0.5 & 0.00 & 0.00 & 0.09 \\
\hline 530 & 0.5 & 0.00 & 0.00 & 0.08 \\
\hline 530 & 0.5 & 0.00 & 0.00 & 0.05 \\
\hline 530 & 1 & 0.01 & 0.00 & 0.15 \\
\hline 530 & 1 & 0.01 & 0.00 & 0.12 \\
\hline 530 & 1 & 0.01 & 0.00 & 0.13 \\
\hline 530 & 3 & 0.01 & 0.00 & 0.09 \\
\hline 530 & 3 & 0.01 & 0.00 & 0.12 \\
\hline 530 & 3 & 0.01 & 0.00 & 0.14 \\
\hline 530 & 7 & 0.01 & 0.00 & 0.12 \\
\hline 530 & 7 & 0.01 & 0.00 & 0.13 \\
\hline
\end{tabular}


CONC. (ppm, blank corrected) FOR 0.45u FILTERED LEACHATES (all test)

\begin{tabular}{|c|c|c|c|c|}
\hline $\mathrm{SA} / \mathrm{V}$ & Duration & & & \\
\hline$(\mathbf{m}-1)$ & (days) & $\mathbf{Y}$ & ZN & ZR \\
\hline 530 & 7 & 0.01 & 0.00 & 0.11 \\
\hline 530 & 14 & 0.02 & 0.00 & 0.16 \\
\hline 530 & 14 & 0.01 & 0.00 & 0.14 \\
\hline 530 & 14 & 0.01 & 0.00 & 0.16 \\
\hline 530 & 28 & 0.02 & 0.00 & 0.25 \\
\hline 530 & 28 & 0.02 & 0.00 & 0.41 \\
\hline 530 & 28 & 0.02 & 0.00 & 0.34 \\
\hline 530 & 56 & 0.02 & 0.00 & 0.23 \\
\hline 530 & 56 & 0.01 & 0.00 & 0.17 \\
\hline 530 & 56 & 0.02 & 0.00 & 0.35 \\
\hline 530 & 91 & 0.02 & 0.00 & 0.23 \\
\hline 530 & 91 & 0.02 & 0.00 & 0.24 \\
\hline 530 & 91 & 0.02 & 0.00 & 0.24 \\
\hline 530 & 182 & 0.02 & 0.01 & 0.25 \\
\hline 530 & 182 & 0.03 & 0.01 & 0.25 \\
\hline 530 & 182 & 0.03 & 0.01 & 0.26 \\
\hline 2000 & 0.25 & 0.00 & 0.00 & 0.02 \\
\hline 2000 & 0.25 & 0.00 & 0.00 & 0.02 \\
\hline 2000 & 0.25 & 0.00 & 0.00 & 0.04 \\
\hline 2000 & 0.5 & 0.00 & 0.00 & 0.04 \\
\hline 2000 & 0.5 & 0.00 & 0.00 & 0.04 \\
\hline 2000 & 0.5 & 0.00 & 0.00 & 0.04 \\
\hline 2000 & 1 & 0.01 & 0.00 & 0.08 \\
\hline 2000 & 1 & 0.01 & 0.00 & 0.07 \\
\hline 2000 & 1 & 0.01 & 0.00 & 0.09 \\
\hline 2000 & 3 & 0.01 & 0.00 & 0.16 \\
\hline 2000 & 3 & 0.01 & 0.01 & 0.14 \\
\hline 2000 & 3 & 0.01 & 0.00 & 0.15 \\
\hline 2000 & 7 & 0.02 & 0.01 & 0.20 \\
\hline 2000 & 7 & 0.02 & 0.01 & 0.24 \\
\hline 2000 & 7 & 0.02 & 0.01 & 0.19 \\
\hline 2000 & 14 & 0.03 & 0.00 & 0.25 \\
\hline 2000 & 14 & 0.02 & 0.00 & 0.26 \\
\hline 2000 & 14 & 0.03 & 0.00 & 0.27 \\
\hline 2000 & 28 & 0.02 & 0.01 & 0.35 \\
\hline 2000 & 28 & 0.04 & 0.01 & 0.33 \\
\hline 2000 & 28 & 0.04 & 0.01 & 0.27 \\
\hline 2000 & 56 & 0.05 & 0.01 & 0.62 \\
\hline 2000 & 56 & 0.04 & 0.01 & 0.57 \\
\hline 2000 & 56 & 0.05 & 0.01 & 0.59 \\
\hline 2000 & 91 & 0.07 & 0.02 & 0.32 \\
\hline 2000 & 91 & 0.07 & 0.01 & 0.21 \\
\hline 2000 & 91 & 0.07 & 0.01 & 0.28 \\
\hline 2000 & 182 & 0.10 & 0.01 & 0.44 \\
\hline 2000 & 182 & 0.11 & 0.01 & 0.06 \\
\hline 2000 & 182 & 0.11 & 0.01 & 0.05 \\
\hline 20000 & 1 & 0.00 & 0.00 & 0.16 \\
\hline
\end{tabular}


CONC. (ppm, blank corrected) FOR 0.45u FILTERED LEACHATES (all test)

\begin{tabular}{|r|r|r|r|r|}
\hline SA/V & \multicolumn{1}{|c|}{ Duration } & & & \\
\hline (m-1) & \multicolumn{1}{|c|}{ (days) } & \multicolumn{1}{|c|}{ Y } & \multicolumn{1}{c|}{ ZN } & \multicolumn{1}{c|}{ ZR } \\
\hline 20000 & 1 & 0.00 & 0.00 & 0.14 \\
\hline 20000 & 1 & 0.00 & 0.00 & 0.17 \\
\hline 20000 & 3 & 0.05 & 0.03 & 0.66 \\
\hline 20000 & 3 & 0.03 & 0.03 & 0.60 \\
\hline 20000 & 3 & 0.04 & 0.02 & 0.63 \\
\hline 20000 & 7 & 0.08 & 0.06 & 1.09 \\
\hline 20000 & 7 & 0.08 & 0.06 & 1.10 \\
\hline 20000 & 7 & 0.08 & 0.05 & 0.97 \\
\hline 20000 & 14 & 0.12 & 0.02 & 1.62 \\
\hline 20000 & 14 & 0.12 & 0.03 & 1.58 \\
\hline 20000 & 14 & 0.15 & 0.03 & 1.56 \\
\hline 20000 & 28 & 0.00 & 0.00 & 0.12 \\
\hline 20000 & 28 & 0.01 & 0.01 & 0.11 \\
\hline 20000 & 28 & 0.00 & 0.23 & 0.22 \\
\hline
\end{tabular}



NORM. CONC. (g/m2, blank corrected) FOR 18A FILTERED LEACHATES (all tests)

\begin{tabular}{|c|c|c|c|c|c|c|c|c|}
\hline & $\overline{\mathbf{A}}$ & $\overline{\mathbf{B}}$ & $\bar{C}$ & $\overline{\mathbf{D}}$ & $\overline{\mathbf{E}}$ & F & $\overline{\mathbf{G}}$ & $\overline{\mathbf{H}}$ \\
\hline$\overline{1}$ & SA/V & Duration & & & & & & \\
\hline 2 & $(m-1)$ & (days) & pH final & $\mathrm{pH}$ in & $\mathrm{AL}$ & $\overline{\mathrm{AS}}$ & $\mathrm{B}$ & $\overline{\mathrm{BA}}$ \\
\hline 3 & 10 & 0.25 & 7.48 & 5.58 & 3.07 & 0.00 & 2.95 & 0.00 \\
\hline 4 & 10 & 0.25 & 7.34 & 5.58 & 3.92 & 0.00 & 3.70 & 0.00 \\
\hline 5 & 10 & 0.25 & 7.27 & 5.58 & 3.70 & 0.00 & 3.91 & 0.00 \\
\hline 6 & 10 & 0.5 & 8.97 & 5.88 & 12.08 & 0.00 & 13.43 & 1.52 \\
\hline 7 & 10 & 0.5 & 9.07 & 5.88 & 16.18 & 0.00 & 17.63 & 2.54 \\
\hline 8 & 10 & 0.5 & 9.03 & 5.88 & 17.25 & 0.00 & 18.80 & 2.54 \\
\hline 9 & 10 & 1 & 9.52 & 5.67 & 29.37 & -132033.49 & 31.55 & 8.68 \\
\hline 10 & 10 & 1 & 9.53 & 5.67 & 29.90 & -132033.49 & 32.13 & 8.68 \\
\hline 11 & 10 & 1 & 9.49 & 5.67 & 26.92 & -132033.49 & 29.46 & 8.68 \\
\hline 12 & 10 & 3 & 9.68 & 5.37 & 62.83 & -132033.49 & 74.31 & 14.89 \\
\hline 13 & 10 & 3 & 9.67 & 5.37 & 60.61 & 0.00 & 68.82 & $\overline{14.89}$ \\
\hline 14 & 10 & 3 & 9.65 & 5.37 & 59.72 & 0.00 & 67.79 & 16.13 \\
\hline$\overline{15}$ & 10 & 7 & 9.55 & 5.74 & 74.42 & 171643.53 & 99.10 & 11.16 \\
\hline 16 & 10 & 7 & 9.51 & 5.74 & 74.87 & 66016.74 & 100.82 & 11.16 \\
\hline 17 & 10 & 7 & 9.51 & 5.74 & 72.64 & 132033.49 & 97.04 & 11.16 \\
\hline 18 & 10 & 14 & 9.72 & 5.74 & 82.44 & -132033.49 & 120.60 & 9.92 \\
\hline 19 & 10 & 14 & 9.71 & 5.74 & 82.89 & -132033.49 & 121.29 & 8.68 \\
\hline 20 & 10 & 14 & 9.71 & 5.74 & 82.89 & -132033.49 & 121.29 & 9.92 \\
\hline 21 & 10 & 28 & 9.34 & 6.01 & 91.09 & 184846.88 & 146.70 & 8.68 \\
\hline 22 & 10 & 28 & 9.33 & 6.01 & 91.09 & 39610.05 & 149.78 & 7.44 \\
\hline 23 & 10 & 28 & 9.30 & 6.01 & 91.09 & 92423.44 & 149.10 & $\overline{8.68}$ \\
\hline 24 & 10 & 56 & 9.43 & 6.01 & 94.34 & 250863.62 & 167.20 & 11.16 \\
\hline 25 & 10 & 56 & 9.43 & 6.01 & 93.45 & 250863.62 & 164.80 & $\pi 1.16$ \\
\hline 26 & 10 & 56 & 9.47 & 6.01 & 94.34 & 105626.79 & 166.86 & 9.92 \\
\hline 27 & 10 & 91 & $9 . \overline{31}$ & 6.01 & 93.58 & 52813.39 & 181.06 & 12.41 \\
\hline 28 & 10 & 91 & 9.38 & 6.01 & 93.58 & 39610.05 & 182.77 & 11.16 \\
\hline 29 & 10 & 91 & 9.30 & 6.01 & 92.25 & 0.00 & 180.37 & 11.16 \\
\hline 30 & 10 & 182 & 9.42 & 6.01 & 94.03 & -132033.49 & 201.02 & $12 . \overline{41}$ \\
\hline 31 & 10 & 182 & 9.43 & 6.01 & 93.14 & -132033.49 & 196.22 & 9.92 \\
\hline 32 & 10 & 182 & 9.48 & 6.01 & 97.59 & 0.00 & 208.22 & $9 . \overline{92}$ \\
\hline 33 & 10 & 364 & 8.64 & 6.00 & 78.61 & $\mid-118830.14$ & 226.43 & 11.16 \\
\hline 34 & 10 & 364 & 8.76 & 6.00 & 87.08 & 171643.53 & 238.09 & 12.41 \\
\hline 35 & 10 & 364 & 8.76 & 6.00 & 87.08 & 171643.53 & 238.09 & 12.41 \\
\hline 36 & 530 & 0.25 & 9.88 & 5.58 & 58.90 & 139955.49 & 104.92 & 1.07 \\
\hline 37 & 530 & 0.25 & 9.90 & 5.58 & 79.69 & 138635.16 & 114.90 & 0.51 \\
\hline 38 & 530 & 0.25 & 9.93 & 5.58 & 78.98 & 209933.24 & 122.53 & 0.51 \\
\hline 39 & 530 & 0.5 & 9.95 & 5.88 & 74.73 & 207292.57 & 144.35 & 0.46 \\
\hline 40 & 530 & 0.5 & 9.97 & 5.88 & 78.61 & -7922.01 & 146.51 & $0 . \overline{96}$ \\
\hline 41 & 530 & 0.5 & 9.96 & 5.88 & 82.84 & 64696.41 & 146.91 & 0.86 \\
\hline$\overline{42}$ & 530 & 1 & 9.95 & 5.74 & $\overline{79.42}$ & 39610.05 & 201.88 & 1.24 \\
\hline$\overline{43}$ & 530 & 1 & 9.95 & 5.74 & 82.04 & -132033.49 & 201.88 & 1.24 \\
\hline 44 & 530 & 1 & 9.95 & 5.74 & 78.98 & -132033.49 & 196.34 & 0.00 \\
\hline 45 & 530 & 3 & 10.03 & 5.51 & $90 . \overline{33}$ & 105626.79 & 309.72 & 0.00 \\
\hline 46 & 530 & 3 & 9.91 & 5.51 & 96.44 & 52813.39 & 315.94 & $1 . \overline{24}$ \\
\hline 47 & 530 & 3 & 10.04 & 5.51 & 93.82 & 118830.14 & 311.45 & 0.00 \\
\hline 48 & 530 & 7 & 10.10 & 5.64 & 85.53 & 52813.39 & 425.03 & -9.92 \\
\hline
\end{tabular}


NORM. CONC. (g/m2, blank corrected) FOR 18A FILTERED LEACHATES (all tests)

\begin{tabular}{|c|c|c|c|c|c|c|c|c|}
\hline & $\overline{\mathbf{A}}$ & $\overline{\mathbf{B}}$ & $\overline{\mathbf{C}}$ & $\overline{\mathbf{D}}$ & $\bar{E}$ & $\overline{\mathbf{F}}$ & $\bar{G}$ & $\mathbf{H}$ \\
\hline 1 & SA/V & Duration & & & & & & \\
\hline 2 & $(m-1)$ & (days) & pH final & $\mathrm{pH}$ in & $\mathbf{A L}$ & $\mathrm{AS}$ & $\mathbf{B}$ & $\mathrm{BA}$ \\
\hline 49 & 530 & 7 & 10.06 & 5.64 & 89.89 & -26406.70 & 425.03 & -11.16 \\
\hline 50 & 530 & 7 & 10.10 & 5.64 & 92.95 & -158440.18 & 411.19 & -11.16 \\
\hline 51 & 530 & 14 & 9.99 & 5.69 & 80.73 & -277270.32 & 511.84 & 0.00 \\
\hline 52 & 530 & 14 & 9.98 & 5.69 & 82.04 & -145236.83 & 501.46 & 2.48 \\
\hline 53 & 530 & 14 & 10.00 & 5.69 & 80.29 & -145236.83 & 494.55 & 0.00 \\
\hline 54 & 530 & 28 & 10.24 & 5.55 & 76.65 & 105626.79 & 540.06 & -0.51 \\
\hline 55 & 530 & 28 & 10.23 & 5.55 & 77.54 & 26406.70 & 543.50 & -0.51 \\
\hline 56 & 530 & 28 & 10.15 & 5.55 & 77.54 & 13203.35 & 536.61 & -0.51 \\
\hline 57 & 530 & 56 & 10.11 & 5.61 & 56.60 & -13203.35 & 589.26 & 0.00 \\
\hline 58 & 530 & 56 & 10.11 & 5.61 & 63.73 & 118830.14 & 589.26 & 0.00 \\
\hline 59 & 530 & 56 & 10.07 & 5.61 & 58.82 & 92423.44 & 592.69 & 0.00 \\
\hline 60 & 530 & 91 & 9.86 & 5.61 & 67.83 & 0.00 & 649.48 & -7.44 \\
\hline 61 & 530 & 91 & 9.90 & 5.61 & 71.39 & 26406.70 & 649.48 & -7.44 \\
\hline 62 & 530 & 91 & 9.88 & 5.61 & 66.93 & -132033.49 & 632.33 & -7.44 \\
\hline 63 & 530 & 182 & 9.98 & 5.61 & 56.60 & 0.00 & 665.25 & 1.24 \\
\hline 64 & 530 & 182 & 9.94 & 5.61 & 45.45 & 0.00 & 699.54 & 0.00 \\
\hline 65 & 530 & 182 & 9.96 & 5.61 & 50.36 & 79220.09 & 692.69 & 0.00 \\
\hline 66 & 2000 & 0.25 & 10.16 & 5.58 & 94.80 & 63376.07 & 214.90 & 0.30 \\
\hline 67 & 2000 & 0.25 & 10.13 & 5.58 & 90.56 & 277270.32 & 213.84 & 0.30 \\
\hline 68 & 2000 & 0.25 & 10.17 & 5.58 & 88.09 & 134674.16 & 213.54 & 0.30 \\
\hline 69 & 2000 & 0.5 & 10.21 & 5.88 & 86.29 & -11883.01 & 289.46 & -0.41 \\
\hline 70 & 2000 & 0.5 & 10.18 & 5.88 & 90.18 & -10562.68 & 280.43 & -0.36 \\
\hline 71 & 2000 & 0.5 & 10.14 & 5.88 & 89.15 & 62055.74 & 243.20 & -0.05 \\
\hline 72 & 2000 & 1 & 10.02 & 5.69 & 98.62 & -39610.05 & 420.43 & 0.00 \\
\hline 73 & 2000 & 1 & 10.09 & 5.69 & 83.78 & -92423.44 & 441.18 & 0.00 \\
\hline 74 & 2000 & 1 & 10.02 & 5.69 & 92.51 & 66016.74 & 455.01 & 0.00 \\
\hline 75 & 2000 & 3 & 10.32 & 5.51 & 79.38 & -448913.85 & 708.81 & 0.00 \\
\hline 76 & 2000 & 3 & 10.30 & 5.51 & 86.36 & -316880.37 & 708.81 & 0.00 \\
\hline 77 & 2000 & 3 & 10.31 & 5.51 & 87.67 & -184846.88 & 705.35 & 0.00 \\
\hline 78 & 2000 & 7 & 10.39 & 5.74 & 83.78 & 92423.44 & 899.24 & 0.00 \\
\hline 79 & 2000 & 7 & 10.37 & 5.74 & 84.66 & 118830.14 & 895.78 & 0.00 \\
\hline 80 & 2000 & 7 & 10.37 & 5.74 & 90.33 & -26406.70 & 885.40 & 0.00 \\
\hline 81 & 2000 & 14 & 10.27 & 5.69 & 82.91 & 66016.74 & 996.08 & -2.48 \\
\hline 82 & 2000 & 14 & 10.30 & 5.69 & 73.31 & 79220.09 & 996.08 & -2.48 \\
\hline 83 & 2000 & 14 & 10.28 & 5.69 & 74.62 & 184846.88 & 978.79 & -2.48 \\
\hline 84 & 2000 & 28 & 10.51 & 5.55 & 71.57 & 0.00 & 1025.55 & -6.20 \\
\hline 85 & 2000 & 28 & 10.56 & 5.55 & 76.20 & 0.00 & 1103.81 & -2.54 \\
\hline 86 & 2000 & 28 & 10.57 & 5.55 & 68.63 & 0.00 & 1079.70 & -2.54 \\
\hline 87 & 2000 & 56 & 10.42 & 5.61 & 64.17 & 52813.39 & 1172.39 & 1.24 \\
\hline 88 & 2000 & 56 & 10.45 & 5.61 & 59.27 & 92423.44 & 1182.68 & 0.00 \\
\hline 89 & 2000 & 56 & 10.39 & 5.61 & 61.94 & 52813.39 & 1141.53 & 1.24 \\
\hline 90 & 2000 & 91 & 10.34 & 5.61 & 83.33 & -92423.44 & 1292.58 & 4.96 \\
\hline 91 & 2000 & 91 & 10.23 & 5.61 & 70.41 & 26406.70 & 1285.72 & 3.72 \\
\hline 92 & 2000 & 91 & 10.23 & 5.61 & 67.74 & 13203.35 & 1210.28 & 2.48 \\
\hline 93 & 2000 & 182 & 10.37 & 5.61 & 61.50 & 145236.83 & 1433.17 & 0.00 \\
\hline 94 & 2000 & 182 & 10.36 & 5.61 & 61.05 & 224456.93 & 1474.32 & 0.00 \\
\hline
\end{tabular}


NORM. CONC. (g/m2, blank corrected) FOR 18A FILTERED LEACHATES (all tests)

\begin{tabular}{|r|r|r|r|r|r|r|r|r|}
\hline & \multicolumn{1}{|c|}{ A } & \multicolumn{1}{c|}{ B } & C & D & E & F & G & H \\
\hline 1 & SA/V & Duration & & & & & & \\
\hline 2 & $(\mathrm{~m}-1)$ & (days) & pH final & pH in & AL & AS & B & BA \\
\hline 95 & 2000 & 182 & 10.32 & 5.61 & 64.62 & 184846.88 & 1395.45 & 2.48 \\
\hline 96 & 20000 & 1 & 10.79 & 5.58 & 41.13 & 1923914.32 & 2117.00 & -0.40 \\
\hline 97 & 20000 & 1 & 10.80 & 5.58 & 45.35 & 496445.91 & 2107.91 & 0.20 \\
\hline 98 & 20000 & 1 & 10.76 & 5.58 & 56.86 & 126836.65 & 2341.41 & 1.07 \\
\hline 99 & 20000 & 3 & 10.91 & 5.51 & 14.40 & -328763.38 & 3595.40 & 0.00 \\
\hline 100 & 20000 & 3 & 10.86 & 5.51 & 17.66 & -328763.38 & 3372.46 & 0.00 \\
\hline 101 & 20000 & 3 & 10.90 & 5.51 & 18.55 & -328763.38 & 3353.30 & 0.00 \\
\hline 102 & 20000 & 7 & 10.86 & 5.64 & 21.38 & -594150.69 & 4043.97 & -6.20 \\
\hline 103 & 20000 & 7 & 10.89 & 5.64 & 29.67 & -858217.66 & 3957.51 & -6.20 \\
\hline 104 & 20000 & 7 & 10.92 & 5.64 & 21.47 & -384085.41 & 3971.76 & -6.20 \\
\hline 105 & 20000 & 14 & 10.96 & 5.64 & 22.47 & 0.00 & 4633.84 & 0.00 \\
\hline 106 & 20000 & 14 & 10.98 & 5.64 & 26.65 & 0.00 & 4488.40 & 0.00 \\
\hline 107 & 20000 & 14 & 10.85 & 5.64 & 25.31 & 0.00 & 4478.20 & 0.00 \\
\hline 108 & 20000 & 28 & 11.21 & 5.55 & 17.83 & 0.00 & 5178.46 & 2.54 \\
\hline 109 & 20000 & 28 & 11.20 & 5.55 & 19.61 & 0.00 & 5212.89 & 0.00 \\
\hline 110 & 20000 & 28 & 11.18 & 5.55 & 28.52 & 290473.67 & 5033.82 & 0.00 \\
\hline
\end{tabular}


NORM. CONC. (g/m2, blank corrected) FOR 18A FILTERED LEACHATES (all tests)

\begin{tabular}{|c|c|c|c|c|c|c|c|c|}
\hline & $\overline{\mathbf{A}}$ & $\mathbf{B}$ & I & $\mathbf{J}$ & $\overline{\mathbf{K}}$ & $\bar{L}$ & $\mathbf{M}$ & $\mathbf{N}$ \\
\hline 1 & $\overline{\mathrm{SA} / \mathrm{V}}$ & Duration & & & & & & \\
\hline 2 & $(m-1)$ & (days) & $\overline{\mathbf{B E}}$ & $\overline{C A}$ & $\overline{C D}$ & CE & $\mathrm{CO}$ & CR \\
\hline 3 & 10 & 0.25 & 0.00 & 0.87 & 0.00 & 3.20 & 0.00 & 0.00 \\
\hline 4 & 10 & 0.25 & 0.00 & 1.51 & 0.00 & 3.20 & 0.00 & 0.00 \\
\hline 5 & 10 & 0.25 & 0.00 & 1.65 & -11423.49 & 0.00 & 0.00 & 0.00 \\
\hline 6 & 10 & 0.5 & 0.00 & 10.48 & -22846.98 & 0.00 & 0.00 & 0.00 \\
\hline 7 & 10 & 0.5 & 0.00 & 14.14 & -22846.98 & 0.00 & 0.00 & 0.00 \\
\hline 8 & 10 & 0.5 & 0.00 & 14.63 & -11423.49 & 0.00 & 0.00 & 0.00 \\
\hline 9 & 10 & 1 & 0.00 & 23.17 & 11423.49 & 0.00 & 0.00 & 0.23 \\
\hline 10 & 10 & 1 & 0.00 & 23.76 & 11423.49 & 0.00 & 0.00 & 0.00 \\
\hline 11 & 10 & 1 & 0.00 & 21.18 & 0.00 & 0.00 & 0.00 & 0.00 \\
\hline 12 & 10 & 3 & 0.00 & 53.93 & 0.00 & 0.00 & 0.00 & -0.23 \\
\hline 13 & 10 & 3 & 0.00 & 51.98 & 0.00 & 0.00 & 0.00 & 0.12 \\
\hline 14 & 10 & 3 & 0.00 & 51.01 & 0.00 & 0.00 & 0.00 & 0.00 \\
\hline 15 & 10 & 7 & 0.00 & 62.67 & 0.00 & 0.00 & 0.00 & 0.12 \\
\hline 16 & 10 & 7 & 0.00 & 63.16 & 0.00 & 0.00 & 0.00 & 0.23 \\
\hline 17 & 10 & 7 & 0.00 & 61.70 & 0.00 & 0.00 & 0.00 & 0.12 \\
\hline 18 & 10 & 14 & 0.00 & 65.54 & 0.00 & 0.00 & 0.00 & 0.35 \\
\hline 19 & 10 & 14 & 0.00 & 65.54 & 0.00 & 0.00 & 0.00 & 0.12 \\
\hline 20 & 10 & 14 & 0.00 & 66.02 & 0.00 & 0.00 & 0.00 & 0.12 \\
\hline 21 & 10 & 28 & 0.00 & 72.19 & 0.00 & 0.00 & -14072.41 & 0.35 \\
\hline 22 & 10 & 28 & 0.00 & 72.19 & 0.00 & 0.00 & -14072.41 & 0.46 \\
\hline 23 & 10 & 28 & 0.00 & 72.68 & -11423.49 & 0.00 & -14072.41 & 0.12 \\
\hline 24 & 10 & 56 & 0.00 & 74.72 & 11423.49 & 0.00 & 14072.41 & 0.69 \\
\hline 25 & 10 & 56 & 0.00 & 74.24 & 0.00 & 0.00 & 0.00 & 0.92 \\
\hline 26 & 10 & 56 & 0.00 & 73.26 & 0.00 & 0.00 & 0.00 & 0.46 \\
\hline 27 & 10 & 91 & 0.00 & 69.96 & 0.00 & 0.00 & 0.00 & 0.46 \\
\hline 28 & 10 & 91 & 0.00 & 73.85 & 0.00 & 0.00 & 0.00 & 0.69 \\
\hline 29 & 10 & 91 & 0.00 & 69.96 & 0.00 & 0.00 & 0.00 & 0.58 \\
\hline 30 & 10 & 182 & 0.00 & 86.48 & 0.00 & 0.00 & 0.00 & 1.61 \\
\hline 31 & 10 & 182 & 0.00 & 85.51 & 0.00 & 0.00 & 14072.41 & 1.15 \\
\hline 32 & 10 & 182 & 0.00 & 88.42 & 0.00 & 0.00 & 0.00 & 0.92 \\
\hline 33 & 10 & 364 & 0.00 & 89.44 & 34270.46 & 3.20 & 0.00 & 0.81 \\
\hline 34 & 10 & 364 & 0.00 & 88.47 & 0.00 & 8.01 & 0.00 & 1.27 \\
\hline 35 & 10 & 364 & 0.00 & 88.47 & 0.00 & 8.01 & 0.00 & 1.27 \\
\hline 36 & 530 & 0.25 & 0.00 & 18.47 & -13708.19 & 0.00 & 0.00 & 0.00 \\
\hline 37 & 530 & 0.25 & 0.00 & 23.55 & -29701.07 & 0.00 & 0.00 & 0.00 \\
\hline 38 & 530 & 0.25 & 0.00 & 20.32 & -14850.53 & 0.00 & 0.00 & 0.00 \\
\hline 39 & 530 & 0.5 & 0.00 & 14.93 & -3427.05 & 0.00 & 0.00 & 0.00 \\
\hline 40 & 530 & 0.5 & 0.00 & 20.62 & 5711.74 & 0.00 & 0.00 & 0.00 \\
\hline 41 & 530 & 0.5 & 0.00 & 22.47 & 14850.53 & 0.00 & 0.00 & 0.00 \\
\hline 42 & 530 & 1 & 0.00 & 11.08 & 0.00 & 0.00 & 0.00 & 0.23 \\
\hline 43 & 530 & 1 & 0.00 & 12.49 & 0.00 & 0.00 & 0.00 & 0.46 \\
\hline 44 & 530 & 1 & 0.00 & 9.13 & 0.00 & 0.00 & 0.00 & 0.23 \\
\hline 45 & 530 & 3 & 0.00 & 6.62 & 0.00 & 0.00 & -14072.41 & 0.58 \\
\hline 46 & 530 & 3 & 0.00 & 5.87 & 0.00 & 0.00 & 0.00 & 0.81 \\
\hline 47 & 530 & 3 & 0.00 & 6.52 & 0.00 & 0.00 & 0.00 & 0.93 \\
\hline 48 & 530 & 7 & 0.00 & 7.02 & 0.00 & 0.00 & 0.00 & 1.97 \\
\hline
\end{tabular}


NORM. CONC. (g/m2, blank corrected) FOR 18A FILTERED LEACHATES (all tests)

\begin{tabular}{|c|c|c|c|c|c|c|c|c|}
\hline & $\overline{\mathbf{A}}$ & $\overline{\mathbf{B}}$ & I & $\mathbf{J}$ & $\mathbf{K}$ & $\mathbf{L}$ & $\mathbf{M}$ & $\overline{\mathbf{N}}$ \\
\hline 1 & SA/V & Duration & & & & & & \\
\hline 2 & $(m-1)$ & (days) & $\mathrm{BE}$ & $\overline{C A}$ & $C D$ & $\overline{C E}$ & $\mathrm{CO}$ & CR \\
\hline 49 & 530 & 7 & 0.00 & 7.47 & 0.00 & 0.00 & 0.00 & 1.86 \\
\hline 50 & 530 & 7 & 0.00 & 7.82 & 0.00 & 0.00 & 0.00 & 2.09 \\
\hline 51 & 530 & 14 & 0.00 & 8.83 & 1153772.24 & 0.00 & 0.00 & 2.20 \\
\hline 52 & 530 & 14 & 0.00 & 11.13 & 0.00 & 0.00 & 0.00 & 2.32 \\
\hline 53 & 530 & 14 & 0.00 & 9.08 & 2273274.02 & 0.00 & 0.00 & 1.97 \\
\hline 54 & 530 & 28 & 0.00 & 14.39 & 0.00 & 0.00 & 0.00 & 3.62 \\
\hline 55 & 530 & 28 & 0.00 & 13.84 & 0.00 & 0.00 & 0.00 & 3.62 \\
\hline$\overline{56}$ & 530 & 28 & 0.00 & 14.73 & 11423.49 & 0.00 & 0.00 & 3.62 \\
\hline 57 & 530 & 56 & 0.00 & 8.55 & 11423.49 & 0.00 & 0.00 & 4.60 \\
\hline 58 & 530 & 56 & 0.00 & 11.71 & 0.00 & 0.00 & 0.00 & 4.37 \\
\hline 59 & 530 & 56 & 0.00 & 8.60 & 11423.49 & 0.00 & 0.00 & 4.37 \\
\hline 60 & 530 & 91 & 0.00 & 16.32 & 0.00 & 0.00 & 0.00 & 5.98 \\
\hline 61 & 530 & 91 & 0.00 & 19.19 & 0.00 & 0.00 & 0.00 & 5.87 \\
\hline 62 & 530 & 91 & 0.00 & 17.78 & 0.00 & 0.00 & 0.00 & 5.75 \\
\hline 63 & 530 & 182 & 0.00 & 24.73 & 11423.49 & 0.00 & 0.00 & 7.14 \\
\hline 64 & 530 & 182 & 0.00 & 24.78 & 0.00 & 0.00 & 0.00 & 7.14 \\
\hline 65 & 530 & 182 & 0.00 & 23.66 & 0.00 & 0.00 & 0.00 & 7.14 \\
\hline 66 & 2000 & 0.25 & 0.00 & 1.84 & 15992.88 & 0.00 & 0.00 & 0.00 \\
\hline 67 & 2000 & 0.25 & 0.00 & 2.77 & -10281.14 & 0.00 & 0.00 & 0.00 \\
\hline$\overline{68}$ & 2000 & 0.25 & 0.00 & 2.77 & -2284.70 & 0.00 & 0.00 & 0.00 \\
\hline 69 & 2000 & 0.5 & 0.00 & 3.23 & -17135.23 & 0.00 & 0.00 & 0.00 \\
\hline 70 & 2000 & 0.5 & 0.00 & 2.62 & -17135.23 & 0.00 & 0.00 & 0.08 \\
\hline 71 & 2000 & 0.5 & 8326.11 & 2.77 & -6854.09 & 0.00 & 0.00 & 0.08 \\
\hline 72 & 2000 & 1 & 0.00 & 1.60 & -11423.49 & 0.00 & 0.00 & 0.70 \\
\hline 73 & 2000 & 1 & 0.00 & 1.10 & -11423.49 & 0.00 & 0.00 & 0.93 \\
\hline 74 & 2000 & 1 & 0.00 & 0.20 & -11423.49 & 0.00 & 0.00 & 1.16 \\
\hline 75 & 2000 & 3 & 0.00 & 2.71 & 0.00 & 0.00 & -14072.41 & 1.62 \\
\hline 76 & 2000 & 3 & 0.00 & 2.91 & 0.00 & 0.00 & -14072.41 & 1.74 \\
\hline 77 & 2000 & 3 & 0.00 & 3.61 & 0.00 & 0.00 & -14072.41 & 2.09 \\
\hline 78 & 2000 & 7 & 0.00 & 8.48 & 0.00 & 0.00 & 0.00 & 4.87 \\
\hline 79 & 2000 & 7 & 0.00 & 7.22 & 0.00 & 0.00 & 0.00 & 4.41 \\
\hline$\overline{80}$ & 2000 & 7 & 0.00 & 7.62 & 0.00 & 0.00 & 0.00 & $\overline{4.41}$ \\
\hline 81 & 2000 & 14 & 0.00 & 8.63 & 2284697.51 & 0.00 & 14072.41 & 5.34 \\
\hline$\overline{82}$ & 2000 & 14 & 0.00 & 7.22 & 0.00 & 0.00 & 0.00 & 5.10 \\
\hline$\overline{83}$ & 2000 & 14 & 0.00 & 7.52 & \#\#\#\#\#\#\# & 0.00 & 0.00 & 4.76 \\
\hline$\overline{84}$ & 2000 & 28 & 0.00 & 10.03 & 0.00 & 0.00 & 0.00 & 7.19 \\
\hline 85 & 2000 & 28 & 0.00 & 10.28 & 0.00 & 0.00 & 0.00 & 6.43 \\
\hline 86 & 2000 & 28 & 0.00 & 12.76 & 0.00 & 0.00 & 0.00 & 6.31 \\
\hline 87 & 2000 & 56 & 0.00 & 15.35 & 0.00 & 0.00 & 0.00 & 10.13 \\
\hline 88 & 2000 & 56 & 0.00 & 13.21 & 0.00 & 0.00 & 0.00 & 10.01 \\
\hline 89 & 2000 & 56 & 0.00 & 16.71 & 0.00 & 0.00 & 0.00 & 10.13 \\
\hline 90 & 2000 & 91 & 0.00 & 28.03 & 0.00 & 0.00 & 0.00 & 14.62 \\
\hline 91 & 2000 & 91 & 0.00 & 52.03 & 0.00 & 0.00 & -14072.41 & 14.04 \\
\hline 92 & 2000 & 91 & 0.00 & 24.83 & 0.00 & 0.00 & -14072.41 & 12.89 \\
\hline 93 & 2000 & 182 & 0.00 & 33.38 & 11423.49 & 0.00 & 14072.41 & 18.76 \\
\hline 94 & 2000 & 182 & 0.00 & 33.86 & 22846.98 & 0.00 & 14072.41 & 19.56 \\
\hline
\end{tabular}


NORM. CONC. (g/m2, blank corrected) FOR 18A FILTERED LEACHATES (all tests)

\begin{tabular}{|c|c|c|c|c|c|c|c|c|}
\hline & $\mathbf{A}$ & B & I & $\mathbf{J}$ & $\bar{K}$ & $\mathbf{L}$ & $\mathbf{M}$ & $\mathbf{N}$ \\
\hline 1 & SA/V & Duration & & & & & & \\
\hline 2 & $(m-1)$ & (days) & $\mathrm{BE}$ & $\overline{\mathrm{CA}}$ & $\overline{C D}$ & $\overline{C E}$ & $\mathrm{CO}$ & $\overline{C R}$ \\
\hline 95 & 2000 & 182 & 0.00 & 38.28 & 11423.49 & 0.53 & 14072.41 & 18.87 \\
\hline 96 & 20000 & 1 & 0.00 & 3.70 & 32119.42 & 0.00 & 0.00 & 1.73 \\
\hline 97 & 20000 & 1 & 0.00 & 3.70 & 59402.14 & 0.00 & $\overline{0.00}$ & 4.21 \\
\hline 98 & 20000 & 1 & 0.00 & 5.93 & 39356.20 & 0.00 & 0.00 & 4.03 \\
\hline 99 & 20000 & 3 & 0.00 & 3.04 & 0.00 & 0.00 & -70080.59 & 20.30 \\
\hline 100 & 20000 & 3 & 0.00 & 3.79 & 0.00 & 0.00 & -70080.59 & 19.77 \\
\hline 101 & 20000 & 3 & 0.00 & 6.80 & 0.00 & 0.00 & -70080.59 & 20.30 \\
\hline 102 & 20000 & 7 & 0.00 & -25.33 & 0.00 & -37.80 & -351810.19 & 36.54 \\
\hline 103 & 20000 & 7 & 0.00 & -24.32 & 0.00 & -37.80 & -351810.19 & 37.12 \\
\hline 104 & 20000 & 7 & 0.00 & -24.67 & 0.00 & -37.80 & -351810.19 & 36.94 \\
\hline 105 & 20000 & 14 & 0.00 & -8.02 & 0.00 & 0.00 & 0.00 & 48.14 \\
\hline 106 & 20000 & 14 & 0.00 & -6.49 & 58145.55 & 0.00 & 0.00 & 51.37 \\
\hline 107 & 20000 & 14 & 0.00 & -10.28 & 0.00 & 0.00 & 0.00 & 51.62 \\
\hline 108 & 20000 & 28 & 0.00 & 21.75 & 0.00 & 0.00 & 0.00 & 52.62 \\
\hline 109 & 20000 & 28 & 0.00 & 22.00 & 0.00 & 0.00 & 0.00 & 53.79 \\
\hline 110 & 20000 & 28 & 0.00 & 0.00 & 0.00 & 0.00 & 309592.96 & 48.29 \\
\hline
\end{tabular}


NORM. CONC. (g/m2, blank corrected) FOR 18A FILTERED LEACHATES (all tests)

\begin{tabular}{|c|c|c|c|c|c|c|c|c|}
\hline & $\overline{\mathbf{A}}$ & $\overline{\mathbf{B}}$ & $\overline{0}$ & $\overline{\mathbf{P}}$ & $\mathbf{Q}$ & $\overline{\mathbf{R}}$ & $\overline{\mathbf{S}}$ & $T$ \\
\hline 1 & SA/V & Duration & & & & & & \\
\hline 2 & $(m-1)$ & (days) & $\mathrm{CS}$ & $\overline{\mathrm{CU}}$ & $\overline{D Y}$ & $\overline{\mathrm{EU}}$ & $\overline{\mathrm{FE}}$ & GD \\
\hline 3 & 10 & 0.25 & 0.00 & 4.17 & 11476.92 & 0.00 & 0.00 & 0.00 \\
\hline 4 & 10 & 0.25 & 0.96 & 3.13 & 11476.92 & 0.00 & 0.00 & 0.00 \\
\hline 5 & 10 & 0.25 & 0.96 & 2.09 & 0.00 & 0.00 & 0.00 & 0.00 \\
\hline 6 & 10 & 0.5 & 11.78 & 2.38 & 0.00 & 0.00 & 0.00 & 0.00 \\
\hline 7 & 10 & 0.5 & 18.85 & 0.00 & 0.00 & 0.00 & 0.00 & 0.00 \\
\hline 8 & 10 & 0.5 & 18.85 & 0.00 & 0.00 & 0.00 & 0.00 & 0.00 \\
\hline 9 & 10 & 1 & 33.73 & -3.13 & 0.00 & 0.00 & 0.00 & 0.00 \\
\hline 10 & 10 & 1 & 38.55 & -3.13 & 0.00 & 0.00 & 0.00 & 0.00 \\
\hline 11 & 10 & 1 & 32.77 & -3.13 & 0.00 & 0.00 & 0.00 & 0.00 \\
\hline 12 & 10 & 3 & 110.84 & 1.04 & 0.00 & 0.00 & 0.00 & -115262.32 \\
\hline 13 & 10 & 3 & 101.20 & 2.09 & 0.00 & 0.00 & 0.00 & -115262.32 \\
\hline 14 & 10 & 3 & 101.20 & 0.00 & 0.00 & 0.00 & 0.00 & 0.00 \\
\hline 15 & 10 & 7 & 149.39 & 1.04 & 0.00 & 0.00 & 0.00 & -115262.32 \\
\hline 16 & 10 & 7 & 130.11 & 3.13 & 0.00 & 0.00 & 0.00 & -115262.32 \\
\hline 17 & 10 & 7 & 130.11 & 2.09 & 0.00 & 0.00 & 0.00 & -115262.32 \\
\hline 18 & 10 & 14 & 139.75 & 2.09 & 0.00 & 0.00 & 0.00 & 23052.46 \\
\hline 19 & 10 & 14 & 149.39 & 2.09 & 0.00 & 0.00 & 0.00 & -207472.18 \\
\hline 20 & 10 & 14 & 139.75 & 1.04 & 0.00 & 0.00 & 0.00 & -92209.86 \\
\hline 21 & 10 & 28 & 159.03 & 2.09 & 0.00 & 0.00 & 0.00 & -115262.32 \\
\hline 22 & 10 & 28 & 159.03 & 2.09 & 0.00 & 0.00 & 0.00 & -115262.32 \\
\hline 23 & 10 & 28 & 168.67 & 2.09 & 0.00 & 0.00 & 0.00 & -115262.32 \\
\hline 24 & 10 & 56 & 187.94 & 5.22 & 0.00 & 0.00 & 0.00 & -115262.32 \\
\hline 25 & 10 & 56 & 187.94 & 4.17 & 0.00 & 0.00 & 0.00 & -230524.64 \\
\hline 26 & 10 & 56 & 187.94 & 3.13 & 0.00 & 0.00 & 0.00 & -115262.32 \\
\hline 27 & 10 & 91 & 216.86 & 0.00 & 0.00 & 0.00 & 0.00 & -230524.64 \\
\hline 28 & 10 & 91 & 216.86 & 2.09 & 0.00 & 0.00 & 0.00 & -230524.64 \\
\hline 29 & 10 & 91 & 216.86 & 0.00 & 0.00 & 0.00 & 0.00 & -230524.64 \\
\hline 30 & 10 & 182 & 236.13 & 0.00 & 0.00 & 0.00 & 0.00 & -230524.64 \\
\hline 31 & 10 & 182 & 216.86 & 0.00 & 0.00 & 0.00 & 0.00 & 0.00 \\
\hline 32 & 10 & 182 & 236.13 & 0.00 & 0.00 & 0.00 & 0.00 & 23052.46 \\
\hline 33 & 10 & 364 & 265.05 & 10.43 & -11476.92 & 0.00 & 0.00 & -103736.09 \\
\hline 34 & 10 & 364 & 284.32 & 12.52 & 0.00 & 0.00 & 0.00 & -230524.64 \\
\hline 35 & 10 & 364 & 284.32 & 12.52 & 0.00 & 0.00 & 0.00 & -230524.64 \\
\hline 36 & 530 & 0.25 & 147.25 & 1.01 & 0.00 & 0.00 & 0.00 & 0.00 \\
\hline 37 & 530 & 0.25 & 170.81 & 0.00 & 0.00 & 0.00 & 0.00 & 0.00 \\
\hline 38 & 530 & 0.25 & 194.37 & 0.00 & 0.00 & 0.00 & 0.00 & 0.00 \\
\hline 39 & 530 & 0.5 & 194.37 & 0.60 & 0.00 & 0.00 & 0.00 & 0.00 \\
\hline 40 & 530 & 0.5 & 206.15 & 0.83 & 0.00 & 0.00 & 0.00 & 0.00 \\
\hline 41 & 530 & 0.5 & 194.37 & 0.83 & 0.00 & 0.00 & 0.00 & 0.00 \\
\hline 42 & 530 & 1 & 147.67 & 0.00 & 0.00 & 0.00 & 0.00 & -115262.32 \\
\hline 43 & 530 & 1 & 155.24 & 0.00 & 0.00 & 0.00 & 0.00 & -115262.32 \\
\hline 44 & 530 & 1 & 140.10 & 0.00 & 0.00 & 0.00 & 0.00 & -115262.32 \\
\hline 45 & 530 & 3 & 185.53 & -1.04 & -11476.92 & 0.00 & 0.00 & -115262.32 \\
\hline 46 & 530 & 3 & 177.96 & 0.00 & -11476.92 & 0.00 & 0.00 & -115262.32 \\
\hline 47 & 530 & 3 & 177.96 & 0.00 & -11476.92 & 0.00 & 0.00 & -115262.32 \\
\hline 48 & 530 & 7 & 193.11 & 3.13 & 0.00 & 0.00 & 0.00 & -230524.64 \\
\hline
\end{tabular}


NORM. CONC. (g/m2, blank corrected) FOR 18A FILTERED LEACHATES (all tests)

\begin{tabular}{|c|c|c|c|c|c|c|c|c|}
\hline & $\overline{\mathbf{A}}$ & B & $\mathbf{0}$ & $\overline{\mathbf{P}}$ & $\overline{\mathbf{Q}}$ & $\mathbf{R}$ & $\mathbf{S}$ & $T$ \\
\hline 1 & SA/V & Duration & & & & & & \\
\hline 2 & $(m-1)$ & (days) & $\mathrm{CS}$ & $\overline{\mathrm{CU}}$ & $\overline{D Y}$ & $\overline{\mathrm{EU}}$ & $\overline{F E}$ & GD \\
\hline 49 & 530 & 7 & 193.11 & 0.00 & 0.00 & 0.00 & 0.00 & -230524.64 \\
\hline 50 & 530 & 7 & 185.53 & 1.04 & 0.00 & 0.00 & 0.00 & -230524.64 \\
\hline 51 & 530 & 14 & 170.39 & 1.04 & 0.00 & 0.00 & 0.00 & -230524.64 \\
\hline 52 & 530 & 14 & 177.96 & 8.35 & 0.00 & 0.00 & 0.00 & 11526.23 \\
\hline 53 & 530 & 14 & 162.82 & 3.13 & 0.00 & 0.00 & 0.00 & -230524.64 \\
\hline 54 & 530 & 28 & 253.27 & 0.00 & 0.00 & 0.00 & 0.00 & -230524.64 \\
\hline 55 & 530 & 28 & 229.71 & 0.00 & 0.00 & 0.00 & 0.00 & -230524.64 \\
\hline 56 & 530 & 28 & 229.71 & 0.00 & 0.00 & 0.00 & 0.00 & -230524.64 \\
\hline 57 & 530 & 56 & 178.31 & 0.00 & 0.00 & 0.00 & 0.00 & -230524.64 \\
\hline 58 & 530 & 56 & 178.31 & 0.00 & 0.00 & 0.00 & 0.00 & -230524.64 \\
\hline 59 & 530 & 56 & 178.31 & 0.00 & 0.00 & 0.00 & 0.00 & -230524.64 \\
\hline 60 & 530 & 91 & 207.22 & 2.09 & 0.00 & 0.00 & 0.00 & -230524.64 \\
\hline 61 & 530 & 91 & 207.22 & 1.04 & 0.00 & 0.00 & 0.00 & -115262.32 \\
\hline 62 & 530 & 91 & 207.22 & 0.00 & 0.00 & 0.00 & 0.00 & -115262.32 \\
\hline 63 & 530 & 182 & 236.13 & 1.04 & 0.00 & 0.00 & 0.00 & -230524.64 \\
\hline 64 & 530 & 182 & 197.58 & 0.00 & 0.00 & 0.00 & 0.00 & -345786.96 \\
\hline 65 & 530 & 182 & 207.22 & 0.00 & 0.00 & 0.00 & 0.00 & -115262.32 \\
\hline 66 & 2000 & 0.25 & 265.05 & 0.60 & 0.00 & 0.00 & 0.00 & 0.00 \\
\hline 67 & 2000 & 0.25 & 241.49 & 0.00 & 0.00 & 0.00 & 0.00 & 0.00 \\
\hline 68 & 2000 & 0.25 & 300.39 & 0.60 & \begin{tabular}{l|l}
0.00 \\
\end{tabular} & 0.00 & 0.00 & 0.00 \\
\hline 69 & 2000 & 0.5 & 229.71 & 0.83 & 0.00 & 0.00 & 0.00 & 0.00 \\
\hline 70 & 2000 & 0.5 & 229.71 & 0.42 & 0.00 & 0.00 & 0.00 & 0.00 \\
\hline 71 & 2000 & 0.5 & 241.49 & 0.66 & 0.00 & 0.00 & 0.00 & 0.00 \\
\hline 72 & 2000 & 1 & 132.52 & 0.00 & 0.00 & 0.00 & 0.00 & -230524.64 \\
\hline 73 & 2000 & 1 & 117.38 & 0.00 & 0.00 & 0.00 & 0.00 & -230524.64 \\
\hline 74 & 2000 & 1 & 140.10 & 1.04 & 0.00 & 0.00 & 0.00 & -230524.64 \\
\hline 75 & 2000 & 3 & 94.66 & 0.00 & 0.00 & 0.00 & 0.00 & -345786.96 \\
\hline 76 & 2000 & 3 & 124.95 & 0.00 & 0.00 & 0.00 & 0.00 & -345786.96 \\
\hline 77 & 2000 & 3 & 94.66 & 3.13 & 0.00 & 0.00 & 0.00 & -345786.96 \\
\hline 78 & 2000 & 7 & 109.81 & 6.26 & 0.00 & 0.00 & 0.00 & -345786.96 \\
\hline 79 & 2000 & 7 & 109.81 & 5.22 & 0.00 & 0.00 & 0.00 & -230524.64 \\
\hline 80 & 2000 & 7 & 109.81 & 4.17 & 0.00 & 0.00 & 0.00 & -345786.96 \\
\hline 81 & 2000 & 14 & 140.10 & 5.22 & 0.00 & 0.00 & 0.00 & 92209.86 \\
\hline 82 & 2000 & 14 & 109.81 & 4.17 & 0.00 & 0.00 & 0.00 & -115262.32 \\
\hline 83 & 2000 & 14 & 117.38 & 0.00 & 0.00 & 0.00 & 0.00 & -115262.32 \\
\hline 84 & 2000 & 28 & 109.81 & 0.00 & 0.00 & 0.00 & 0.00 & -461049.28 \\
\hline 85 & 2000 & 28 & 194.37 & 0.00 & 0.00 & 0.00 & 0.00 & -461049.28 \\
\hline 86 & 2000 & 28 & 170.81 & 0.60 & 0.00 & 0.00 & 0.00 & -461049.28 \\
\hline 87 & 2000 & 56 & 149.39 & 8.35 & 0.00 & 0.00 & 0.00 & -461049.28 \\
\hline 88 & 2000 & 56 & 139.75 & 0.00 & 0.00 & 0.00 & 0.00 & -461049.28 \\
\hline 89 & 2000 & 56 & 130.11 & 13.56 & 0.00 & 0.00 & 0.00 & -461049.28 \\
\hline 90 & 2000 & 91 & 216.86 & 34.42 & 0.00 & 0.00 & 0.00 & -230524.64 \\
\hline 91 & 2000 & 91 & 207.22 & 16.69 & 0.00 & 0.00 & 0.00 & -461049.28 \\
\hline 92 & 2000 & 91 & 207.22 & 14.60 & 0.00 & 0.00 & 0.00 & -461049.28 \\
\hline 93 & 2000 & 182 & 265.05 & 3.13 & 0.00 & 0.00 & 0.00 & -115262.32 \\
\hline 94 & 2000 & 182 & 284.32 & 0.00 & 0.00 & 0.00 & 0.00 & -230524.64 \\
\hline
\end{tabular}


NORM. CONC. (g/m2, blank corrected) FOR 18A FILTERED LEACHATES (all tests)

\begin{tabular}{|c|c|c|c|c|c|c|c|c|}
\hline & $\overline{\mathbf{A}}$ & $\overline{\mathbf{B}}$ & $\overline{0}$ & $\overline{\mathbf{P}}$ & $\mathbf{Q}$ & $\overline{\mathbf{R}}$ & $\overline{\mathbf{S}}$ & $\mathbf{T}$ \\
\hline 1 & $\mathrm{SA} / \mathrm{V}$ & Duration & & & & & & \\
\hline 2 & $(m-1)$ & (days) & $\mathrm{CS}$ & $\mathrm{CU}$ & DY & EU & $\overline{F E}$ & $\overline{G D}$ \\
\hline 95 & 2000 & 182 & 274.69 & 18.78 & 0.00 & 0.00 & 0.00 & 34578.70 \\
\hline 96 & 20000 & 1 & 183.86 & 19.09 & 0.00 & 0.00 & 0.00 & 0.00 \\
\hline 97 & 20000 & 1 & 188.48 & 14.31 & 0.00 & 0.00 & 0.00 & 0.00 \\
\hline 98 & 20000 & 1 & 214.55 & 31.43 & 0.00 & 0.00 & 0.00 & 0.00 \\
\hline 99 & 20000 & 3 & 147.75 & 0.00 & 0.00 & 0.00 & 0.00 & $\# \# \# \# \# \#$ \\
\hline 100 & 20000 & 3 & 148.90 & 35.55 & 0.00 & 0.00 & 0.00 & $\# \# \# \# \# \# \#$ \\
\hline 101 & 20000 & 3 & 125.03 & 0.00 & 0.00 & 0.00 & 0.00 & 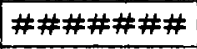 \\
\hline 102 & 20000 & 7 & 166.60 & -15.65 & -229538.46 & -57896.81 & -57896.81 & $\# \# \# \# \#$ \\
\hline 103 & 20000 & 7 & 227.18 & 57.37 & -229538.46 & -57896.81 & -57896.81 & \#\#\#\#\#\#\# \\
\hline 104 & 20000 & 7 & 206.39 & -15.51 & -229538.46 & -57896.81 & -57896.81 & 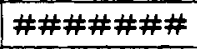 \\
\hline 105 & 20000 & 14 & 170.39 & 0.00 & 0.00 & 0.00 & 0.00 & \#\#\#\#\#\# \\
\hline 106 & 20000 & 14 & 154.52 & 0.00 & 0.00 & 0.00 & 0.00 & $\# \# \# \# \#$ \\
\hline 107 & 20000 & 14 & 132.52 & 0.00 & 0.00 & 0.00 & 0.00 & \#\#\#\#\#\# \\
\hline 108 & 20000 & 28 & 147.25 & 0.00 & 0.00 & 0.00 & 0.00 & 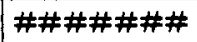 \\
\hline 109 & 20000 & 28 & 153.14 & 0.00 & 0.00 & 0.00 & 0.00 & \#\#\#\#\#\#\# \\
\hline 110 & 20000 & 28 & 126.05 & 0.00 & 126246.15 & 0.00 & 0.00 & $\# \# \# \# \# \#$ \\
\hline
\end{tabular}


NORM. CONC. (g/m2, blank corrected) FOR 18A FILTERED LEACHATES (all tests)

\begin{tabular}{|c|c|c|c|c|c|c|c|c|}
\hline & $\mathbf{A}$ & B & $\mathbf{U}$ & $\mathbf{v}$ & $\mathbf{W}$ & $\bar{X}$ & $\bar{Y}$ & $\mathbf{Z}$ \\
\hline 1 & SA/V & Duration & & & & & & \\
\hline 2 & $(m-1)$ & (days) & $\mathbf{K}$ & LA & LI & MG & $\mathrm{MN}$ & $\mathrm{MO}$ \\
\hline 3 & 10 & 0.25 & 84321.52 & 0.41 & 3.17 & 0.43 & 0.93 & 2.68 \\
\hline 4 & 10 & 0.25 & 0.00 & 0.21 & 4.02 & 1.94 & 0.93 & 2.68 \\
\hline 5 & 10 & 0.25 & 0.00 & 0.00 & 3.72 & 0.00 & 0.93 & 2.68 \\
\hline 6 & 10 & 0.5 & 0.00 & 0.00 & 12.15 & 7.02 & 0.99 & 0.00 \\
\hline 7 & 10 & 0.5 & 0.00 & 0.00 & 16.14 & 8.29 & 0.99 & 15.58 \\
\hline 8 & 10 & 0.5 & 0.00 & 0.00 & 17.07 & 9.99 & 0.99 & 16.73 \\
\hline 9 & 10 & 1 & 0.00 & 0.00 & 29.94 & 20.89 & 1.86 & 27.33 \\
\hline 10 & 10 & 1 & 0.00 & 0.00 & 29.94 & 21.96 & 0.93 & 27.33 \\
\hline 11 & 10 & 1 & 0.00 & 0.00 & 29.45 & 19.81 & 0.93 & 23.58 \\
\hline 12 & 10 & 3 & 0.00 & 0.00 & 74.39 & 35.10 & 2.79 & 71.80 \\
\hline 13 & 10 & 3 & 0.00 & 0.00 & 67.68 & 42.42 & 2.79 & 66.98 \\
\hline 14 & 10 & 3 & 0.00 & 0.00 & 67.68 & 43.07 & 1.86 & 64.83 \\
\hline 15 & 10 & 7 & 0.00 & 0.00 & 99.39 & 8.18 & 2.79 & 93.77 \\
\hline 16 & 10 & 7 & 0.00 & 0.00 & 101.22 & 8.40 & 1.86 & 95.38 \\
\hline 17 & 10 & 7 & 0.00 & 0.00 & 97.56 & 6.68 & 1.86 & 90.02 \\
\hline 18 & 10 & 14 & 0.00 & 0.00 & 118.90 & 4.74 & 2.79 & 115.74 \\
\hline 19 & 10 & 14 & 0.00 & 0.00 & 121.34 & 2.58 & 2.79 & 117.35 \\
\hline 20 & 10 & 14 & 0.00 & 0.00 & 123.17 & 1.29 & 2.79 & 117.88 \\
\hline 21 & 10 & 28 & 0.00 & 0.00 & 147.56 & 0.65 & 1.86 & 138.24 \\
\hline 22 & 10 & 28 & 0.00 & 0.00 & 151.83 & 4.95 & 2.79 & 141.46 \\
\hline 23 & 10 & 28 & 0.00 & 0.00 & 151.22 & 3.23 & 2.79 & 138.78 \\
\hline 24 & 10 & 56 & 0.00 & 0.00 & 166.46 & 8.40 & 4.65 & 160.75 \\
\hline 25 & 10 & 56 & 0.00 & 0.00 & 166.46 & 7.32 & 4.65 & 157.53 \\
\hline 26 & 10 & 56 & 0.00 & 0.00 & 168.29 & 4.95 & 3.72 & 158.60 \\
\hline 27 & 10 & 91 & 0.00 & -2.06 & 180.36 & 3.23 & 6.52 & 172.00 \\
\hline 28 & 10 & 91 & 0.00 & -2.06 & 179.75 & 4.74 & 5.59 & 174.14 \\
\hline 29 & 10 & 91 & 0.00 & -2.06 & 179.14 & 0.22 & 4.65 & 170.39 \\
\hline 30 & 10 & 182 & 1047996.01 & 0.00 & 198.53 & 11.84 & 7.45 & 190.22 \\
\hline 31 & 10 & 182 & 2144175.75 & 0.62 & 194.27 & 16.58 & 8.38 & 187.54 \\
\hline 32 & 10 & 182 & -843215.18 & 0.82 & 205.85 & 11.41 & 7.45 & 197.18 \\
\hline 33 & 10 & 364 & 0.00 & 0.82 & 227.44 & 13.57 & 8.38 & 223.44 \\
\hline 34 & 10 & 364 & 0.00 & 1.23 & 241.46 & 18.09 & 10.24 & 231.48 \\
\hline 35 & 10 & 364 & 0.00 & 1.23 & 241.46 & 18.09 & 10.24 & 231.48 \\
\hline 36 & 530 & 0.25 & 0.00 & 0.34 & 114.55 & 18.79 & 1.48 & 69.71 \\
\hline 37 & 530 & 0.25 & -368605.49 & 0.00 & 128.42 & 25.06 & 0.00 & 115.41 \\
\hline 38 & 530 & 0.25 & -368605.49 & 0.00 & 136.54 & 18.79 & 0.00 & 119.16 \\
\hline 39 & 530 & 0.5 & 368605.49 & 0.50 & 163.44 & 14.09 & 2.08 & 147.84 \\
\hline 40 & 530 & 0.5 & 368605.49 & 0.67 & 165.68 & 15.67 & 3.56 & 154.24 \\
\hline 41 & 530 & 0.5 & 368605.49 & 0.00 & 168.74 & 17.22 & 3.07 & 159.03 \\
\hline 42 & 530 & 1 & 0.00 & 0.00 & 218.25 & 0.00 & 0.00 & 206.44 \\
\hline 43 & 530 & 1 & 0.00 & 0.00 & 217.04 & 0.00 & 0.99 & 209.44 \\
\hline 44 & 530 & 1 & 0.00 & 0.00 & 209.23 & 0.00 & 0.00 & 201.04 \\
\hline 45 & 530 & 3 & 0.00 & -0.21 & 321.78 & 1.58 & 0.99 & 328.27 \\
\hline 46 & 530 & 3 & 0.00 & -0.21 & 325.39 & 2.57 & 0.99 & 333.67 \\
\hline 47 & 530 & 3 & 0.00 & -0.21 & 319.97 & 1.58 & 0.00 & 331.87 \\
\hline 48 & 530 & 7 & 0.00 & 0.00 & 442.27 & 5.13 & 1.98 & 446.49 \\
\hline
\end{tabular}


NORM. CONC. (g/m2, blank corrected) FOR 18A FILTERED LEACHATES (all tests)

\begin{tabular}{|c|c|c|c|c|c|c|c|c|}
\hline & $\mathbf{A}$ & B & $\overline{\mathbf{U}}$ & $\bar{V}$ & $\mathbf{W}$ & $\overline{\mathbf{X}}$ & $\bar{Y}$ & $\overline{\mathbf{Z}}$ \\
\hline 1 & SA/V & Duration & & & & & & \\
\hline 2 & $(m-1)$ & (days) & $\mathbf{K}$ & LA & $\mathrm{LI}$ & MG & MN & $\mathrm{MO}$ \\
\hline 49 & 530 & 7 & 0.00 & 0.00 & 441.66 & 0.00 & 0.00 & 445.29 \\
\hline 50 & 530 & 7 & 0.00 & 0.00 & 427.84 & 1.78 & 0.99 & 432.09 \\
\hline 51 & 530 & 14 & 0.00 & 0.00 & 504.43 & 1.97 & 1.98 & 511.31 \\
\hline 52 & 530 & 14 & 0.00 & 0.63 & 493.61 & 11.45 & 8.90 & 496.90 \\
\hline 53 & 530 & 14 & 0.00 & 0.00 & 485.79 & 3.75 & 2.97 & 497.50 \\
\hline 54 & 530 & 28 & 0.00 & 0.00 & 549.21 & 0.00 & -0.99 & 544.73 \\
\hline 55 & 530 & 28 & 0.00 & 0.00 & 547.47 & 0.00 & -0.99 & 544.15 \\
\hline 56 & 530 & 28 & 0.00 & 0.00 & 543.42 & 0.00 & 0.99 & 546.46 \\
\hline 57 & 530 & 56 & 0.00 & 0.00 & 646.09 & -0.86 & 0.00 & 551.90 \\
\hline 58 & 530 & 56 & 0.00 & 0.00 & 652.19 & 4.31 & 0.93 & 557.26 \\
\hline 59 & 530 & 56 & 0.00 & 0.00 & 652.19 & 2.80 & 0.93 & 557.26 \\
\hline 60 & 530 & 91 & 530020.97 & 0.00 & 725.23 & 4.95 & 1.86 & 610.31 \\
\hline 61 & 530 & 91 & 0.00 & 0.00 & 731.33 & 0.22 & 0.93 & 610.31 \\
\hline 62 & 530 & 91 & 0.00 & 0.00 & 713.04 & -1.08 & 0.93 & 599.59 \\
\hline 63 & 530 & 182 & 0.00 & 0.00 & 762.18 & 4.52 & 0.93 & 616.20 \\
\hline 64 & 530 & 182 & 0.00 & 0.00 & 810.96 & 0.00 & 0.00 & 621.56 \\
\hline 65 & 530 & 182 & 0.00 & 0.00 & 804.87 & 3.23 & 0.93 & 632.27 \\
\hline 66 & 2000 & 0.25 & 184302.75 & 0.00 & 281.33 & 3.15 & 0.99 & 232.43 \\
\hline 67 & 2000 & 0.25 & 0.00 & 0.00 & 275.75 & 6.27 & 0.00 & 224.41 \\
\hline 68 & 2000 & 0.25 & 368605.49 & 0.00 & 273.52 & 3.15 & 0.00 & 224.99 \\
\hline 69 & 2000 & 0.5 & 921513.73 & 0.34 & 346.53 & -1.57 & 2.08 & 280.27 \\
\hline 70 & 2000 & 0.5 & 368605.49 & 0.00 & 333.14 & -1.57 & 3.56 & 271.21 \\
\hline 71 & 2000 & 0.5 & 737210.99 & 0.00 & 291.55 & 1.57 & 1.48 & 238.78 \\
\hline 72 & 2000 & 1 & 0.00 & 0.00 & 487.06 & 1.58 & 0.00 & 453.09 \\
\hline 73 & 2000 & 1 & 0.00 & 0.00 & 504.49 & 0.00 & 0.00 & 464.50 \\
\hline 74 & 2000 & 1 & 0.00 & 0.00 & 522.53 & 0.59 & 0.99 & 484.90 \\
\hline 75 & 2000 & 3 & 0.00 & 0.00 & 763.08 & -1.18 & 0.00 & 737.55 \\
\hline 76 & 2000 & 3 & 0.00 & 0.00 & 763.08 & -1.18 & 0.00 & 737.55 \\
\hline 77 & 2000 & 3 & 0.00 & 0.00 & 757.07 & 1.18 & 2.97 & 755.56 \\
\hline 78 & 2000 & 7 & 0.00 & 0.00 & 949.94 & 5.13 & 3.96 & 886.98 \\
\hline 79 & 2000 & 7 & 0.00 & 0.00 & 949.94 & 2.17 & 2.97 & 892.99 \\
\hline 80 & 2000 & 7 & 0.00 & 0.00 & 931.91 & 1.18 & 1.98 & 880.98 \\
\hline 81 & 2000 & 14 & 0.00 & 0.63 & 1046.02 & 6.71 & 3.96 & 990.21 \\
\hline 82 & 2000 & 14 & 0.00 & 0.00 & 1046.02 & 4.54 & 2.97 & 978.20 \\
\hline 83 & 2000 & 14 & 0.00 & 0.00 & 1021.97 & -0.20 & 0.99 & 966.20 \\
\hline 84 & 2000 & 28 & 0.00 & 0.00 & 1142.04 & 0.00 & 0.99 & 1038.22 \\
\hline 85 & 2000 & 28 & 0.00 & 0.00 & 1203.21 & 0.00 & 2.97 & 1061.76 \\
\hline 86 & 2000 & 28 & 0.00 & 0.00 & 1174.28 & 0.00 & 12.86 & 998.28 \\
\hline 87 & 2000 & 56 & 0.00 & 0.21 & 1378.03 & 11.20 & 6.52 & 1034.14 \\
\hline 88 & 2000 & 56 & 0.00 & 0.00 & 1396.32 & 1.72 & 0.93 & 1044.86 \\
\hline 89 & 2000 & 56 & 0.00 & 0.00 & 1335.35 & 12.27 & 6.52 & 1012.71 \\
\hline 90 & 2000 & 91 & 0.00 & 5.56 & 1554.85 & 34.02 & 26.06 & 1161.13 \\
\hline 91 & 2000 & 91 & 0.00 & 2.06 & 1530.46 & 17.44 & 12.10 & 1150.42 \\
\hline 92 & 2000 & 91 & 0.00 & 1.44 & 1451.20 & 15.29 & 12.10 & 1086.12 \\
\hline 93 & 2000 & 182 & 0.00 & 0.00 & 1768.27 & 5.38 & 2.79 & 1318.13 \\
\hline 94 & 2000 & 182 & 0.00 & 0.00 & 1810.95 & 3.88 & 0.93 & 1355.64 \\
\hline
\end{tabular}


NORM. CONC. (g/m2, blank corrected) FOR 18A FILTERED LEACHATES (all tests)

\begin{tabular}{|c|c|c|c|c|c|c|c|c|}
\hline & $\overline{\mathbf{A}}$ & $\overline{\mathbf{B}}$ & $\overline{\mathbf{U}}$ & $\overline{\mathbf{V}}$ & $\overline{\mathbf{W}}$ & $\bar{X}$ & $\bar{Y}$ & $\overline{\mathbf{Z}}$ \\
\hline 1 & $\mathrm{SA} / \mathrm{V}$ & Duration & & & & & & \\
\hline 2 & $(m-1)$ & (days) & $\mathbf{K}$ & LA & LI & MG & $\mathrm{MN}$ & $\mathrm{MO}$ \\
\hline 95 & 2000 & 182 & 0.00 & 3.29 & 1731.68 & 20.67 & 13.96 & 1280.62 \\
\hline 96 & 20000 & 1 & 0.00 & 0.00 & 2377.79 & 18.86 & -10.28 & 2011.32 \\
\hline 97 & 20000 & 1 & 0.00 & 0.00 & 2444.76 & 25.08 & -1.98 & 2003.96 \\
\hline 98 & 20000 & 1 & 0.00 & 0.00 & 2737.57 & 42.06 & -0.37 & 2252.77 \\
\hline 99 & 20000 & 3 & 0.00 & 0.00 & 3755.89 & -1.97 & 0.00 & 3417.72 \\
\hline 100 & 20000 & 3 & 0.00 & 0.00 & 3609.56 & -1.97 & 4.21 & 3218.24 \\
\hline 101 & 20000 & 3 & 0.00 & 0.00 & 3575.53 & -1.97 & 4.95 & 3207.68 \\
\hline 102 & 20000 & 7 & -138.17 & -4.19 & 4292.49 & -40.47 & 0.00 & 3825.80 \\
\hline 103 & 20000 & 7 & -138.17 & -3.14 & 4202.30 & -8.88 & 34.62 & 3705.77 \\
\hline 104 & 20000 & 7 & -138.17 & -4.19 & 4219.20 & -40.47 & 0.13 & 3710.15 \\
\hline 105 & 20000 & 14 & 0.00 & 0.00 & 4930.09 & 0.00 & 0.00 & 4020.84 \\
\hline 106 & 20000 & 14 & 0.00 & 0.00 & 4774.01 & 0.00 & 0.00 & 3971.03 \\
\hline 107 & 20000 & 14 & 0.00 & 0.00 & 4749.72 & 0.00 & 0.00 & 3870.81 \\
\hline 108 & 20000 & 28 & 0.00 & -1.05 & 5142.63 & 8.50 & 34.62 & 4789.46 \\
\hline 109 & 20000 & 28 & 0.00 & 0.00 & 5171.56 & 12.75 & 9.89 & 4760.61 \\
\hline 110 & 20000 & 28 & 0.00 & 4.82 & 4919.29 & 2.34 & 68.24 & 4532.10 \\
\hline
\end{tabular}


NORM. CONC. (g/m2, blank corrected) FOR 18A FILTERED LEACHATES (all tests)

\begin{tabular}{|c|c|c|c|c|c|c|c|c|}
\hline & $\bar{A}$ & $\overline{\mathbf{B}}$ & $\overline{\mathbf{A A}}$ & $\overline{\mathbf{A B}}$ & $\overline{A C}$ & $\overline{\mathbf{A D}}$ & $\overline{A E}$ & AF \\
\hline 1 & $\overline{S A} / \mathrm{V}$ & Duration & & & & & & \\
\hline 2 & $(m-1)$ & (days) & NA & ND & NI & $P$ & PB & RU \\
\hline 3 & 10 & 0.25 & 3.19 & 0.00 & 0.22 & -183313.12 & 32316.71 & 0.00 \\
\hline 4 & 10 & 0.25 & 3.68 & 0.00 & 0.22 & -183313.12 & 0.00 & 0.00 \\
\hline 5 & 10 & 0.25 & 3.14 & 0.00 & 0.00 & -183313.12 & 0.00 & 0.00 \\
\hline 6 & 10 & 0.5 & 11.94 & 0.00 & 0.00 & 0.00 & 0.00 & 0.00 \\
\hline 7 & 10 & 0.5 & 15.96 & 0.00 & 0.00 & 0.00 & 0.00 & 0.00 \\
\hline 8 & 10 & 0.5 & 16.77 & 0.00 & 0.00 & 0.00 & 0.00 & $\overline{0.00}$ \\
\hline 9 & 10 & 1 & 28.71 & 0.00 & 0.00 & 68742.42 & 0.00 & 0.00 \\
\hline 10 & 10 & 1 & 29.25 & 0.00 & 0.00 & 0.00 & 0.00 & 0.00 \\
\hline 11 & 10 & 1 & 25.61 & 0.00 & 0.00 & 0.00 & 0.00 & 0.00 \\
\hline 12 & 10 & 3 & 68.75 & 0.00 & 0.00 & 68742.42 & 0.00 & 0.00 \\
\hline 13 & 10 & 3 & 63.89 & 0.00 & 1.08 & -45828.28 & 0.00 & 0.00 \\
\hline 14 & 10 & 3 & 64.43 & 0.00 & 0.00 & -435368.67 & 0.00 & 0.00 \\
\hline 15 & 10 & 7 & 94.56 & 0.00 & 0.65 & -229141.40 & -215444.76 & 0.00 \\
\hline 16 & 10 & 7 & 96.99 & 0.00 & 0.65 & 320797.96 & -107722.38 & 0.00 \\
\hline 17 & 10 & 7 & 93.89 & 0.00 & 0.00 & -229141.40 & -215444.76 & 0.00 \\
\hline 18 & 10 & 14 & 116.87 & 0.00 & 1.51 & 779080.77 & -215444.76 & 26332.24 \\
\hline 19 & 10 & 14 & 117.27 & 0.00 & 0.86 & 412454.53 & -215444.76 & 0.00 \\
\hline 20 & 10 & 14 & 117.68 & 0.00 & 0.86 & 297883.82 & -323167.14 & 0.00 \\
\hline 21 & 10 & 28 & 133.11 & 0.00 & 0.86 & -229141.40 & -323167.14 & -13166.12 \\
\hline$\overline{22}$ & 10 & 28 & 137.29 & 0.00 & 0.86 & 45828.28 & -323167.14 & -13166.12 \\
\hline 23 & 10 & 28 & 135.94 & 0.00 & 0.65 & -229141.40 & -323167.14 & -13166.12 \\
\hline 24 & 10 & 56 & 152.32 & 0.00 & 1.08 & 206227.26 & -215444.76 & 52664.49 \\
\hline 25 & 10 & 56 & 150.97 & 0.00 & 0.86 & 595767.65 & -215444.76 & 0.00 \\
\hline 26 & 10 & 56 & 153.67 & 0.00 & 1.08 & 68742.42 & -323167.14 & 0.00 \\
\hline 27 & 10 & 91 & 171.19 & 0.00 & 0.22 & 389540.39 & -430889.52 & 0.00 \\
\hline 28 & 10 & 91 & 172.54 & 0.00 & 1.08 & 22914.14 & -430889.52 & 0.00 \\
\hline 29 & 10 & 91 & 169.85 & 0.00 & 0.86 & 0.00 & -430889.52 & 0.00 \\
\hline 30 & 10 & 182 & 193.45 & 0.00 & 0.22 & 1489419.12 & -107722.38 & 0.00 \\
\hline 31 & 10 & 182 & 188.06 & 0.78 & 0.00 & 1122792.88 & 0.00 & 26332.24 \\
\hline 32 & 10 & 182 & 198.84 & 0.00 & 0.00 & 847823.19 & -107722.38 & -131661.22 \\
\hline$\overline{33}$ & 10 & 364 & 205.55 & 1.56 & 3.67 & 1626903.96 & 226217.00 & -26332.24 \\
\hline 34 & 10 & 364 & 216.34 & 1.30 & 3.02 & 1901873.65 & 193900.28 & 13166.12 \\
\hline 35 & 10 & 364 & 216.34 & 1.30 & 3.02 & 1901873.65 & 193900.28 & 13166.12 \\
\hline 36 & 530 & 0.25 & 105.74 & 0.00 & 0.42 & 323089.38 & 0.00 & 4.44 \\
\hline 37 & 530 & 0.25 & 123.53 & 0.00 & -1.68 & -128319.19 & -391032.24 & 0.00 \\
\hline 38 & 530 & 0.25 & 129.73 & 0.00 & -1.25 & 453699.98 & -260688.16 & 0.00 \\
\hline 39 & 530 & 0.5 & 152.81 & 0.00 & 0.85 & 515568.16 & 0.00 & 2.22 \\
\hline 40 & 530 & 0.5 & 153.72 & 0.00 & 0.00 & 128319.19 & 0.00 & 6.67 \\
\hline 41 & 530 & 0.5 & 156.62 & 0.00 & 0.85 & 515568.16 & -130344.08 & 4.44 \\
\hline$\overline{42}$ & 530 & 1 & 191.70 & 0.00 & 0.00 & 641595.93 & -430889.52 & 0.00 \\
\hline 43 & 530 & 1 & 192.98 & 0.00 & 0.00 & 412454.53 & -430889.52 & 0.00 \\
\hline$\overline{44}$ & 530 & 1 & 186.56 & 0.00 & 0.00 & 343712.11 & -430889.52 & 0.00 \\
\hline$\overline{45}$ & 530 & 3 & 287.00 & 0.00 & -0.45 & 847823.19 & -398572.81 & 0.00 \\
\hline 46 & 530 & 3 & 293.42 & 0.00 & 0.45 & 1145707.02 & -506295.19 & 0.00 \\
\hline 47 & 530 & 3 & 289.57 & 0.00 & 0.22 & 733252.49 & -398572.81 & 0.00 \\
\hline 48 & 530 & 7 & 399.99 & 0.00 & 1.34 & 1535247.40 & -538611.90 & 0.00 \\
\hline
\end{tabular}


NORM. CONC. (g/m2, blank corrected) FOR 18A FILTERED LEACHATES (all tests)

\begin{tabular}{|c|c|c|c|c|c|c|c|c|}
\hline & $\overline{\mathbf{A}}$ & $\overline{\mathbf{B}}$ & $\overline{\mathbf{A A}}$ & $\overline{\mathbf{A B}}$ & $\overline{\mathrm{AC}}$ & $\overline{A D}$ & $\overline{\mathrm{AE}}$ & $\overline{A F}$ \\
\hline 1 & SA/V & Duration & & & & & & \\
\hline 2 & $(m-1)$ & (days) & NA & ND & NI & $\mathbf{P}$ & PB & $\mathrm{RU}$ \\
\hline 49 & 530 & 7 & 399.99 & 0.00 & 0.00 & 1076964.60 & -538611.90 & 0.00 \\
\hline 50 & 530 & 7 & 387.15 & 0.00 & 0.00 & 1168621.16 & -538611.90 & 0.00 \\
\hline 51 & 530 & 14 & 460.03 & 0.00 & 0.89 & \begin{tabular}{|l|}
1603989.82 \\
\end{tabular} & -754056.66 & 0.00 \\
\hline 52 & 530 & 14 & 451.05 & 0.00 & 3.80 & 1810217.09 & -646334.28 & 579309.39 \\
\hline 53 & 530 & 14 & 444.63 & 0.00 & 1.56 & \begin{tabular}{|l|}
2268499.89 \\
\end{tabular} & -754056.66 & 157993.47 \\
\hline 54 & 530 & 28 & 527.69 & 0.00 & 0.00 & 1535247.40 & -107722.38 & 0.00 \\
\hline 55 & 530 & 28 & 527.69 & 0.00 & 0.00 & 1443590.84 & -107722.38 & 0.00 \\
\hline 56 & 530 & 28 & 523.65 & 0.00 & 0.00 & \begin{tabular}{|l|}
695646.39 \\
\end{tabular} & -107722.38 & 0.00 \\
\hline 57 & 530 & 56 & 561.93 & 0.00 & -0.22 & \begin{tabular}{|l|}
1489419.12 \\
\end{tabular} & -646334.28 & 0.00 \\
\hline 58 & 530 & 56 & 564.63 & 0.00 & 0.00 & 1924787.79 & -538611.90 & 0.00 \\
\hline 59 & 530 & 56 & 570.02 & 0.00 & 0.00 & 2245585.75 & -538611.90 & 0.00 \\
\hline 60 & 530 & 91 & 644.91 & 0.00 & 0.43 & 2360156.45 & -538611.90 & 0.00 \\
\hline 61 & 530 & 91 & 644.91 & 0.00 & 0.86 & 2291414.03 & -538611.90 & 0.00 \\
\hline 62 & 530 & 91 & 630.09 & 0.00 & 0.22 & 1489419.12 & -538611.90 & 0.00 \\
\hline 63 & 530 & 182 & 646.71 & 0.00 & 0.00 & 1810217.09 & 129266.86 & 39498.37 \\
\hline 64 & 530 & 182 & 680.41 & 0.00 & 0.00 & 2016444.35 & -107722.38 & 0.00 \\
\hline 65 & 530 & 182 & 672.32 & 0.00 & 0.43 & 2383070.60 & 32316.71 & 13166.12 \\
\hline 66 & 2000 & 0.25 & 247.46 & 0.00 & -1.68 & 1741474.67 & 0.00 & 0.00 \\
\hline 67 & 2000 & 0.25 & 244.29 & 0.00 & -0.83 & 708046.94 & 521376.32 & 0.00 \\
\hline 68 & 2000 & 0.25 & 243.24 & 0.00 & 0.00 & 1225906.51 & 130344.08 & 2.22 \\
\hline 69 & 2000 & 0.5 & 309.82 & 0.00 & 1.27 & 320797.96 & 0.00 & -0.08 \\
\hline 70 & 2000 & 0.5 & 301.26 & 0.00 & 0.42 & 838657.54 & 0.00 & -2.22 \\
\hline 71 & 2000 & 0.5 & 268.42 & 0.00 & 0.42 & 774497.94 & 0.00 & 6.75 \\
\hline 72 & 2000 & 1 & 395.73 & 0.00 & 0.00 & 1581075.68 & -538611.90 & 0.00 \\
\hline 73 & 2000 & 1 & 409.85 & 0.00 & 0.22 & 1191535.30 & -646334.28 & 0.00 \\
\hline 74 & 2000 & 1 & 421.40 & 0.00 & 0.22 & 1649818.10 & -538611.90 & 0.00 \\
\hline 75 & 2000 & 3 & 646.34 & 0.00 & -0.89 & 1214449.44 & -969501.42 & 0.00 \\
\hline 76 & 2000 & 3 & 647.62 & 0.00 & -0.89 & 1191535.30 & -969501.42 & 0.00 \\
\hline 77 & 2000 & 3 & 639.92 & 0.00 & 0.67 & 1970616.07 & -861779.04 & 0.00 \\
\hline 78 & 2000 & 7 & 792.60 & 0.00 & 2.01 & 2314328.17 & -861779.04 & 0.00 \\
\hline 79 & 2000 & 7 & 790.03 & 0.00 & 1.34 & 2933009.96 & -861779.04 & 0.00 \\
\hline 80 & 2000 & 7 & 781.05 & 0.00 & 1.12 & 2405984.74 & -861779.04 & 0.00 \\
\hline 81 & 2000 & 14 & 882.02 & 0.00 & 2.46 & 2955924.10 & -969501.42 & 618807.76 \\
\hline 82 & 2000 & 14 & 879.45 & 0.00 & 1.79 & 2887181.68 & -969501.42 & 0.00 \\
\hline 83 & 2000 & 14 & 861.48 & 0.00 & 0.22 & 2955924.10 & -969501.42 & 39498.37 \\
\hline 84 & 2000 & 28 & 934.70 & 0.00 & 0.00 & 2589297.86 & -107722.38 & 0.00 \\
\hline 85 & 2000 & 28 & 1031.31 & 0.00 & 1.48 & 1741474.67 & -107722.38 & 0.00 \\
\hline 86 & 2000 & 28 & 1005.70 & 0.00 & 1.91 & 1329020.14 & -107722.38 & 0.00 \\
\hline 87 & 2000 & 56 & 1101.76 & 0.00 & 3.67 & 3712090.73 & -861779.04 & 0.00 \\
\hline 88 & 2000 & 56 & 1113.89 & 0.00 & 0.00 & 3964146.28 & -969501.42 & 0.00 \\
\hline 89 & 2000 & 56 & 1073.45 & 0.00 & 3.24 & 3528777.61 & -969501.42 & 0.00 \\
\hline 90 & 2000 & 91 & 1258.18 & 4.67 & 17.69 & 4009974.56 & 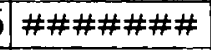 & 0.00 \\
\hline 91 & 2000 & 91 & 1248.74 & 0.26 & 8.20 & 4239115.96 & 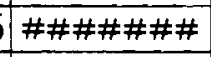 & 0.00 \\
\hline 92 & 2000 & 91 & 1182.69 & 0.00 & 7.12 & 4353686.66 & \#\#\#\#\#\#\# & 0.00 \\
\hline 93 & 2000 & 182 & 1371.52 & 0.00 & 1.08 & 4995282.59 & 247761.47 & 65830.61 \\
\hline 94 & 2000 & 182 & 1385.00 & 0.00 & 0.00 & 5041110.87 & 247761.47 & 39498.37 \\
\hline
\end{tabular}


NORM. CONC. (g/m2, blank corrected) FOR 18A FILTERED LEACHATES (all tests)

\begin{tabular}{|c|c|c|c|c|c|c|c|c|}
\hline & $\mathbf{A}$ & B & $\mathbf{A A}$ & $\mathbf{A B}$ & AC & AD & $\mathbf{A E}$ & $\mathbf{A F}$ \\
\hline 1 & $\mathrm{SA} / \mathrm{V}$ & Duration & & & & & & \\
\hline 2 & $(m-1)$ & (days) & NA & ND & NI & $\mathbf{P}$ & PB & $\mathrm{RU}$ \\
\hline 95 & 2000 & 182 & 1325.68 & 1.56 & 10.35 & 4949454.31 & 301622.67 & 92162.86 \\
\hline 96 & 20000 & 1 & 1879.82 & 0.00 & 0.01 & 4128607.94 & 1875.02 & 0.00 \\
\hline 97 & 20000 & 1 & 1907.50 & 0.00 & 0.00 & 6452621.92 & 1564128.96 & 0.00 \\
\hline 98 & 20000 & 1 & 2129.58 & 0.00 & 6.70 & 5170479.53 & $\# \# \# \# \# \# \#$ & 0.00 \\
\hline 99 & 20000 & 3 & 2901.80 & 0.00 & -2.22 & 3322550.35 & 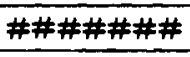 & 0.00 \\
\hline 100 & 20000 & 3. & 2749.42 & 0.00 & -2.22 & 6735382.41 & $\# \# \# \# \# \# \#$ & 0.00 \\
\hline 101 & 20000 & 3. & 2734.91 & 0.00 & -2.22 & 5041110.87 & \#\#\#\#\#\#\# & 0.00 \\
\hline 102 & 20000 & 7 & 3281.43 & -17.01 & -6.70 & 4811969.47 & $\# \# \# \# \# \# \#$ & 0.00 \\
\hline 103 & 20000 & 7 & 3236.50 & -17.01 & 16.74 & 6759671.40 & \#\#\#\#\#\#\# & 0.00 \\
\hline 104 & 20000 & 7 & 3241.92 & -17.01 & -7.81 & 5541326.56 & 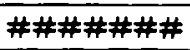 & 0.00 \\
\hline 105 & 20000 & 14 & 3697.13 & 0.00 & 0.00 & 8707373.33 & \#\#\#\#\#\#\# & 0.00 \\
\hline 106 & 20000 & 14 & 3574.41 & 0.00 & 0.00 & 8772220.35 & \#\#\#\#\#\#\# & 0.00 \\
\hline 107 & 20000 & 14 & 3588.00 & 0.00 & 0.00 & 9051085.43 & \#\#\#\#\#\#\# & 0.00 \\
\hline 108 & 20000 & 28 & 4220.73 & 0.00 & 0.00 & 4926540.17 & 0.00 & 0.00 \\
\hline 109 & 20000 & 28 & 4227.47 & 0.00 & 0.00 & 6072247.19 & 0.00 & 0.00 \\
\hline 110 & 20000 & 28 & 4095.37 & 0.00 & 0.00 & \#\#\#\#\#\#\# & 0.00 & 18.10 \\
\hline
\end{tabular}


NORM. CONC. (g/m2, blank corrected) FOR 18A FILTERED LEACHATES (all tests)

\begin{tabular}{|c|c|c|c|c|c|c|c|c|}
\hline & $\mathbf{A}$ & B & $\mathbf{A G}$ & AH & AI & $\mathbf{A J}$ & AK & AL \\
\hline 1 & SA/V & Duration & & & & & & \\
\hline 2 & $(m-1)$ & (days) & $\overline{S B}$ & SI & SR & TE & $\mathrm{TI}$ & $\mathrm{V}$ \\
\hline 3 & 10 & 0.25 & 0.00 & 2.59 & 1.48 & 0.00 & 0.00 & 0.00 \\
\hline 4 & 10 & 0.25 & 0.00 & 3.30 & 1.48 & 0.00 & 0.00 & 0.00 \\
\hline 5 & 10 & 0.25 & 0.00 & 3.48 & 1.48 & 125078.37 & 0.00 & 0.00 \\
\hline 6 & 10 & 0.5 & 0.00 & 12.38 & 10.35 & 0.00 & 0.00 & 0.00 \\
\hline 7 & 10 & 0.5 & 0.00 & 16.12 & 13.30 & 0.00 & 0.00 & 0.00 \\
\hline 8 & 10 & 0.5 & 0.00 & 17.06 & 13.30 & 0.00 & 0.00 & 0.00 \\
\hline 9 & 10 & 1 & 0.00 & 27.38 & 20.70 & 0.00 & 0.00 & 0.00 \\
\hline 10 & 10 & 1 & 0.00 & 28.55 & 22.17 & 0.00 & 0.00 & 0.00 \\
\hline 11 & 10 & 1) & 0.00 & 26.09 & 20.70 & 0.00 & 0.00 & 0.00 \\
\hline 12 & 10 & 3 & 59856.26 & 63.28 & 48.78 & 0.00 & 0.00 & 0.00 \\
\hline 13 & 10 & 3 & 71827.52 & 59.11 & 47.30 & 12507.84 & 0.00 & 0.00 \\
\hline 14 & 10 & 3 & 47885.01 & 58.28 & 47.30 & 0.00 & 0.00 & 0.00 \\
\hline 15 & 10 & 7 & 119712.53 & 81.21 & 56.17 & -162601.88 & 0.00 & 0.00 \\
\hline 16 & 10 & 7 & 107741.27 & 82.46 & 56.17 & -37523.51 & 0.00 & 0.00 \\
\hline 17 & 10 & 7 & 119712.53 & 79.54 & 54.70 & -162601.88 & 0.00 & 0.00 \\
\hline 18 & 10 & 14 & 0.00 & 96.72 & 56.17 & -125078.37 & 0.00 & 0.00 \\
\hline 19 & 10 & 14 & 0.00 & 97.13 & 56.17 & -125078.37 & 0.00 & 0.00 \\
\hline 20 & 10 & 14) & 0.00 & 97.55 & 56.17 & -250156.74 & 0.00 & 0.00 \\
\hline 21 & 10 & 28 & 71827.52 & 110.90 & 57.65 & -125078.37 & 0.00 & 0.00 \\
\hline 22 & 10 & 28 & 23942.51 & 112.99 & 56.17 & -125078.37 & 0.00 & 0.00 \\
\hline 23 & 10 & 28 & 35913.76 & 112.57 & 57.65 & -125078.37 & 0.00 & 0.00 \\
\hline 24 & 10 & 56 & 119712.53 & 124.20 & 59.13 & 87554.86 & 0.00 & 14711.24 \\
\hline 25 & 10 & 56 & 95770.02 & 122.11 & 57.65 & 62539.18 & 0.00 & 0.00 \\
\hline 26 & 10 & 56 & 47885.01 & 123.78 & 57.65 & 0.00 & 0.00 & 0.00 \\
\hline 27 & 10 & 91 & 0.00 & 133.29 & 59.13 & -375235.11 & 0.00 & 0.00 \\
\hline 28 & 10 & 91 & 11971.25 & 134.12 & 60.61 & -375235.11 & 0.00 & 0.00 \\
\hline 29 & 10 & 91 & 0.00 & 133.29 & 59.13 & -250156.74 & 0.00 & 0.00 \\
\hline 30 & 10 & 182 & 11971.25 & 146.78 & 57.65 & -250156.74 & 8.34 & 0.00 \\
\hline 31 & 10 & 182 & 0.00 & 143.86 & 57.65 & -125078.37 & 0.00 & 0.00 \\
\hline 32 & 10 & 182 & 0.00 & 152.62 & 57.65 & -250156.74 & 0.00 & 0.00 \\
\hline 33 & 10 & 364 & 323223.82 & 162.92 & 59.13 & -75047.02 & 0.00 & 14711.24 \\
\hline 34 & 10 & 364 & 0.00 & 170.84 & 57.65 & -200125.39 & 0.00 & 14711.24 \\
\hline 35 & 10 & 364 & 0.00 & 170.84 & 57.65 & -200125.39 & 0.00 & 14711.24 \\
\hline 36 & 530 & 0.25 & 0.00 & 88.51 & 14.63 & 1992498.43 & 0.00 & 0.00 \\
\hline 37 & 530 & 0.25 & 0.00 & 98.15 & 16.26 & -397749.22 & 0.00 & 0.00 \\
\hline 38 & 530 & 0.25 & 0.00 & 102.91 & 14.93 & 0.00 & 0.00 & 0.00 \\
\hline 39 & 530 & 0.5 & -208299.79 & 115.37 & 7.10 & -796749.22 & 0.00 & 0.00 \\
\hline 40 & 530 & 0.5 & -208299.79 & 117.94 & 12.27 & 0.00 & 0.00 & 0.00 \\
\hline 41 & 530 & 0.5 & 0.00 & 119.49 & 13.30 & 796749.22 & 0.00 & 0.00 \\
\hline 42 & 530 & 1 & 35913.76 & 139.33 & 5.07 & -375235.11 & 0.00 & 0.00 \\
\hline 43 & 530 & 1 & 0.00 & 139.33 & 6.76 & -250156.74 & 0.00 & 0.00 \\
\hline 44 & 530 & 1 & 59856.26 & 135.47 & 5.07 & -250156.74 & 0.00 & 0.00 \\
\hline 45 & 530 & 3 & 191540.04 & 195.89 & 3.38 & -125078.37 & 0.00 & 0.00 \\
\hline 46 & 530 & 3 & 203511.29 & 201.04 & 3.38 & -250156.74 & 0.00 & 0.00 \\
\hline 47 & 530 & 3 & 191540.04 & 198.46 & 3.38 & -125078.37 & 0.00 & 14711.24 \\
\hline 48 & 530 & 7 & 227453.80 & 242.66 & 5.07 & -125078.37 & 0.00 & 14711.24 \\
\hline
\end{tabular}


NORM. CONC. (g/m2, blank corrected) FOR 18A FILTERED LEACHATES (all tests)

\begin{tabular}{|c|c|c|c|c|c|c|c|c|}
\hline & $\mathbf{A}$ & B & $\mathbf{A G}$ & AH & AI & $\mathbf{A J}$ & AK & AL \\
\hline 1 & SA $/ V$ & Duration & & & & & & \\
\hline 2 & $(m-1)$ & (days) & SB & SI & SR & TE & TI & V \\
\hline 49 & 530 & 7 & 203511.29 & 243.09 & 5.07 & -250156.74 & 0.00 & 0.00 \\
\hline 50 & 530 & 7 & 155626.28 & 239.23 & 5.07 & -250156.74 & 0.00 & 0.00 \\
\hline 51 & 530 & 14 & 275338.81 & 281.67 & 5.07 & -125078.37 & 0.00 & 0.00 \\
\hline 52 & 530 & 14 & 287310.06 & 277.82 & 6.76 & -250156.74 & 0.00 & 0.00 \\
\hline 53 & 530 & 14 & 155626.28 & 273.53 & 5.07 & -375235.11 & 0.00 & 0.00 \\
\hline 54 & 530 & 28 & 478850.10 & 290.75 & 8.87 & -375235.11 & 0.00 & 0.00 \\
\hline 55 & 530 & 28 & 263367.56 & 289.90 & 7.39 & -500313.48 & 0.00 & 0.00 \\
\hline 56 & 530 & 28 & 0.00 & 287.78 & 7.39 & -500313.48 & 0.00 & 0.00 \\
\hline 57 & 530 & 56 & 11971.25 & 289.00 & 4.43 & 25015.67 & 0.00 & 0.00 \\
\hline 58 & 530 & 56 & 11971.25 & 289.84 & 4.43 & 137586.21 & 0.00 & 14711.24 \\
\hline 59 & 530 & 56 & 191540.04 & 289.84 & 2.96 & 100062.70 & 0.00 & 14711.24 \\
\hline 60 & 530 & 91 & 347166.32 & 322.54 & 8.87 & 187617.55 & 0.00 & 44133.72 \\
\hline 61 & 530 & 91 & 359137.58 & 322.13 & 8.87 & 300188.09 & 0.00 & 29422.48 \\
\hline 62 & 530 & 91 & 311252.57 & 314.62 & 10.35 & 187617.55 & 0.00 & 14711.24 \\
\hline 63 & 530 & 182 & 371108.83 & 335.66 & 11.83 & -250156.74 & 0.00 & 29422.48 \\
\hline 64 & 530 & 182 & 191540.04 & 344.00 & 11.83 & -375235.11 & 0.00 & 0.00 \\
\hline 65 & 530 & 182 & 335195.07 & 343.17 & 10.35 & -250156.74 & 0.00 & 14711.24 \\
\hline 66 & 2000 & 0.25 & 208299.79 & 158.04 & 0.74 & 1593498.43 & 0.00 & 0.00 \\
\hline 67 & 2000 & 0.25 & 104149.90 & 156.12 & 0.44 & 1593498.43 & 0.00 & 0.00 \\
\hline 68 & 2000 & 0.25 & 208299.79 & 156.76 & 0.74 & 1593498.43 & 0.00 & 0.00 \\
\hline 69 & 2000 & 0.5 & 0.00 & 189.02 & 0.89 & 1194498.43 & 0.00 & 0.00 \\
\hline 70 & 2000 & 0.5 & -104149.90 & 186.97 & 1.18 & 0.00 & 0.00 & 0.00 \\
\hline 71 & 2000 & 0.5 & 208299.79 & 173.47 & 1.48 & -399000.00 & 0.00 & 0.00 \\
\hline 72 & 2000 & 1 & 143655.03 & 237.37 & 0.00 & 237648.90 & 0.00 & 0.00 \\
\hline 73 & 2000 & 1 & 83798.77 & 239.09 & 0.00 & 212633.23 & 0.00 & 0.00 \\
\hline 74 & 2000 & 1 & 155626.28 & 249.37 & 0.00 & 262664.58 & 0.00 & 0.00 \\
\hline 75 & 2000 & 3 & 59856.26 & 340.81 & 1.69 & -250156.74 & 0.00 & 0.00 \\
\hline 76 & 2000 & 3 & 0.00 & 342.96 & 1.69 & -125078.37 & 0.00 & 0.00 \\
\hline 77 & 2000 & 3 & 155626.28 & 342.53 & 1.69 & 0.00 & 0.00 & 0.00 \\
\hline 78 & 2000 & 7 & 239425.05 & 392.29 & 3.38 & 225141.07 & 0.00 & 14711.24 \\
\hline 79 & 2000 & 7 & 239425.05 & 391.86 & 3.38 & 212633.23 & 0.00 & 29422.48 \\
\hline 80 & 2000 & 7 & 239425.05 & 391.00 & 3.38 & 412758.62 & 0.00 & 29422.48 \\
\hline 81 & 2000 & 14 & 335195.07 & 433.02 & 5.07 & 487805.64 & 0.00 & 29422.48 \\
\hline 82 & 2000 & 14 & 335195.07 & 428.73 & 3.38 & 437774.29 & 0.00 & 29422.48 \\
\hline 83 & 2000 & 14 & 347166.32 & 420.15 & 3.38 & -125078.37 & 0.00 & 14711.24 \\
\hline 84 & 2000 & 28 & 514763.86 & 437.09 & 6.76 & 187617.55 & 22.24 & 0.00 \\
\hline 85 & 2000 & 28 & 454907.60 & 459.13 & 5.91 & -125078.37 & 0.00 & 0.00 \\
\hline 86 & 2000 & 28 & 454907.60 & 450.63 & 5.91 & -250156.74 & 0.00 & 0.00 \\
\hline 87 & 2000 & 56 & 215482.55 & 454.56 & 5.91 & 400250.78 & 0.00 & 29422.48 \\
\hline 88 & 2000 & 56 & 203511.29 & 454.56 & 4.43 & 412758.62 & 0.00 & 29422.48 \\
\hline 89 & 2000 & 56 & 383080.08 & 446.22 & 5.91 & 250156.74 & 0.00 & 29422.48 \\
\hline 90 & 2000 & 91 & 263367.56 & 504.53 & 14.78 & -112570.53 & 8.34 & 58844.96 \\
\hline 91 & 2000 & 91 & 263367.56 & 500.36 & 16.26 & -237648.90 & 0.00 & 58844.96 \\
\hline 92 & 2000 & 91 & 263367.56 & 479.51 & 10.35 & -100062.70 & 0.00 & 44133.72 \\
\hline 93 & 2000 & 182 & 814045.17 & 571.31 & 14.78 & -625391.85 & 0.00 & 88267.44 \\
\hline 94 & 2000 & 182 & 694332.65 & 583.82 & 14.78 & -750470.22 & 0.00 & 73556.20 \\
\hline
\end{tabular}


NORM. CONC. (g/m2, blank corrected) FOR 18A FILTERED LEACHATES (all tests)

\begin{tabular}{|c|c|c|c|c|c|c|c|c|}
\hline & $\bar{A}$ & B & $\overline{A G}$ & $\overline{\mathrm{AH}}$ & AI & AJ & AK & AL \\
\hline 1 & $\overline{\mathrm{SA} / \mathrm{V}}$ & Duration & & & & & & \\
\hline 2 & $(m-1)$ & (days) & SB & SI & SR & TE & $\mathrm{TI}$ & $\bar{V}$ \\
\hline 95 & 2000 & 182 & 634476.39 & 558.80 & 19.22 & -125078.37 & 0.00 & 73556.20 \\
\hline 96 & 20000 & 1 & 834698.94 & 747.16 & 0.00 & 6381880.42 & 0.00 & 0.00 \\
\hline 97 & 20000 & 1 & 833199.18 & 751.98 & 0.00 & 9560990.60 & 0.00 & 0.00 \\
\hline 98 & 20000 & 1 & -107274.39 & 848.29 & 0.00 & 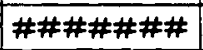 & 0.00 & 0.00 \\
\hline 99 & 20000 & 3 & 0.00 & 947.34 & 0.00 & 1751097.18 & 0.00 & 0.00 \\
\hline 100 & 20000 & 3 & 0.00 & 900.26 & 0.00 & 2451035.74 & 0.00 & 0.00 \\
\hline 101 & 20000 & 3 & 0.00 & 906.61 & 0.00 & 2126332.29 & 0.00 & 0.00 \\
\hline 102 & 20000 & 7 & -359137.58 & 1014.95 & -8.45 & 7942476.49 & -27.80 & -220668.60 \\
\hline 103 & 20000 & 7 & -538706.37 & 1006.38 & -8.45 & 7192006.27 & -27.80 & -220668.60 \\
\hline 104 & 20000 & 7 & -338906.16 & 1010.58 & -8.45 & 6809641.69 & -27.80 & -220668.60 \\
\hline 105 & 20000 & 14 & 0.00 & 1153.13 & 0.00 & 5753605.02 & 0.00 & 0.00 \\
\hline 106 & 20000 & 14 & 1218673.51 & 1121.52 & 0.00 & 6939473.04 & 0.00 & 0.00 \\
\hline 107 & 20000 & 14 & 0.00 & 1135.98 & 0.00 & 3752351.10 & 0.00 & 0.00 \\
\hline 108 & 20000 & 28 & 1256981.52 & 1229.17 & 7.39 & 2313949.84 & 0.00 & 2.94 \\
\hline 109 & 20000 & 28 & 1496406.57 & 1231.30 & 7.39 & 1626018.81 & 0.00 & 0.00 \\
\hline 110 & 20000 & 28 & 1711889.12 & 1202.38 & 0.00 & 4127586.21 & 0.00 & 0.00 \\
\hline
\end{tabular}


NORM. CONC. (g/m2, blank corrected) FOR 18A FILTERED LEACHATES (all tests)

\begin{tabular}{|c|c|c|c|c|c|}
\hline & $\bar{A}$ & $\bar{B}$ & $\overline{\mathbf{A M}}$ & $\overline{\mathrm{AN}}$ & AO \\
\hline 1 & SA/V & Duration & & & \\
\hline 2 & $(m-1)$ & (days) & $\bar{Y}$ & $\mathrm{ZN}$ & ZR \\
\hline 3 & 10 & 0.25 & 0.00 & -236504.51 & 0.00 \\
\hline 4 & 10 & 0.25 & 0.00 & -236504.51 & 0.00 \\
\hline 5 & 10 & 0.25 & 0.00 & -236504.51 & 0.00 \\
\hline 6 & 10 & 0.5 & 0.00 & 0.00 & 0.00 \\
\hline 7 & 10 & 0.5 & 0.00 & 12447.61 & 0.00 \\
\hline 8 & 10 & 0.5 & 0.00 & 0.00 & 0.00 \\
\hline 9 & 10 & 1 & 0.00 & 0.00 & 0.00 \\
\hline 10 & 10 & 1 & 0.00 & 0.00 & 0.00 \\
\hline$\overline{11}$ & 10 & 1 & 0.00 & 0.00 & 0.00 \\
\hline 12 & 10 & 3 & 0.00 & 0.00 & 0.00 \\
\hline 13 & 10 & 3 & 0.00 & 0.00 & 0.00 \\
\hline 14 & 10 & 3 & 0.00 & 0.00 & 0.00 \\
\hline 15 & 10 & 7 & 0.00 & -37342.82 & 0.00 \\
\hline 16 & 10 & 7 & $\overline{0.00}$ & -24895.21 & 0.24 \\
\hline 17 & 10 & 7 & 0.00 & -37342.82 & 0.00 \\
\hline 18 & 10 & 14 & 0.00 & -12447.61 & 0.00 \\
\hline 19 & 10 & 14 & 0.00 & -12447.61 & 0.00 \\
\hline 20 & 10 & 14 & 0.00 & -12447.61 & 0.00 \\
\hline 21 & 10 & 28 & 0.00 & -24895.21 & -2.89 \\
\hline 22 & 10 & 28 & 0.00 & -12447.61 & -2.41 \\
\hline 23 & 10 & 28 & 0.00 & 12447.61 & -2.41 \\
\hline 24 & 10 & 56 & 0.00 & -435666.21 & 0.24 \\
\hline 25 & 10 & 56 & 0.00 & -124476.06 & 0.00 \\
\hline 26 & 10 & 56 & 0.00 & -460561.42 & 0.00 \\
\hline 27 & 10 & 91 & 0.00 & 0.00 & 1.45 \\
\hline 28 & 10 & 91 & 0.00 & 0.00 & 2.65 \\
\hline 29 & 10 & 91 & 0.00 & 0.00 & 1.21 \\
\hline 30 & 10 & 182 & 0.00 & 0.00 & 0.00 \\
\hline 31 & 10 & 182 & 0.00 & 0.00 & 0.00 \\
\hline 32 & 10 & 182 & 0.00 & 0.00 & 0.00 \\
\hline 33 & 10 & 364 & 0.00 & 12447.61 & 0.00 \\
\hline 34 & 10 & 364 & 0.00 & 0.00 & 0.00 \\
\hline 35 & 10 & 364 & 0.00 & 0.00 & 0.00 \\
\hline 36 & 530 & 0.25 & 0.00 & \#\#\#\#\#\# & 0.00 \\
\hline 37 & 530 & 0.25 & 0.00 & \#\#\#\#\#\#\# & 0.00 \\
\hline 38 & 530 & 0.25 & 0.00 & \#\#\#\#\#\# & 0.00 \\
\hline 39 & 530 & 0.5 & 0.00 & \#\#\#\#\#\#\# & 0.00 \\
\hline 40 & 530 & 0.5 & 0.00 & \#\#\#\#\#\#\# & 0.00 \\
\hline$\overline{41}$ & 530 & 0.5 & 0.00 & 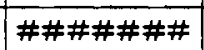 & 0.00 \\
\hline 42 & 530 & 1 & 0.00 & \#\#\#\#\#\#\# & 0.00 \\
\hline 43 & 530 & 1 & 0.00 & \#\#\#\#\#\# & 0.00 \\
\hline 44 & 530 & 1 & 0.00 & \#\#\#\#\#\# & 0.00 \\
\hline 45 & 530 & 3 & 0.00 & -858884.81 & 0.25 \\
\hline 46 & 530 & 3 & 0.00 & 211609.30 & $1 . \overline{27}$ \\
\hline 47 & 530 & 3 & 0.00 & -161818.88 & 1.27 \\
\hline 48 & 530 & 7 & 0.00 & -946018.05 & 0.51 \\
\hline
\end{tabular}


NORM. CONC. (g/m2, blank corrected) FOR 18A FILTERED LEACHATES (all tests)

\begin{tabular}{|c|c|c|c|c|c|}
\hline & $\overline{\mathbf{A}}$ & $\overline{\mathbf{B}}$ & $\overline{\mathbf{A M}}$ & $\overline{A N}$ & $\mathbf{A O}$ \\
\hline 1 & SA/V & Duration & & & \\
\hline 2 & $(m-1)$ & (days) & $\mathbf{Y}$ & $\mathbf{Z N}$ & ZR \\
\hline 49 & 530 & 7 & 0.00 & -485456.63 & $\overline{0.00}$ \\
\hline $\mathbf{5 0}$ & 530 & 7 & 0.00 & -833989.60 & 0.00 \\
\hline 51 & 530 & 14 & 0.00 & 4456242.92 & 1.53 \\
\hline 52 & 530 & 14 & 0.00 & 2638892.46 & 2.29 \\
\hline 53 & 530 & 14 & 0.00 & 2464625.98 & 0.76 \\
\hline 54 & 530 & 28 & 0.00 & 572589.87 & 0.23 \\
\hline 55 & 530 & 28 & 0.00 & 3398196.42 & 0.00 \\
\hline 56 & 530 & 28 & 0.00 & 2352597.52 & 0.00 \\
\hline 57 & 530 & 56 & 0.00 & 3423091.63 & -0.24 \\
\hline 58 & 530 & 56 & 0.00 & 1132732.14 & -0.24 \\
\hline 59 & 530 & 56 & 0.00 & 1867140.89 & -0.24 \\
\hline 60 & 530 & 91 & 0.00 & 1095389.32 & 1.93 \\
\hline 61 & 530 & 91 & 0.00 & -62238.03 & 1.45 \\
\hline 62 & 530 & 91 & 0.00 & 298742.54 & 0.72 \\
\hline 63 & 530 & 182 & 0.00 & 2004064.56 & 0.96 \\
\hline 64 & 530 & 182 & 0.00 & 4057919.53 & 0.00 \\
\hline 65 & 530 & 182 & 0.00 & 4767433.07 & 0.48 \\
\hline 66 & 2000 & 0.25 & 0.00 & $\# \# \# \# \# \# \#$ & 0.00 \\
\hline 67 & 2000 & 0.25 & 0.00 & $\# \# \# \# \# \# \#$ & 0.00 \\
\hline 68 & 2000 & 0.25 & 0.00 & -495414.72 & 0.00 \\
\hline 69 & 2000 & 0.5 & 0.00 & -436910.97 & 0.00 \\
\hline 70 & 2000 & 0.5 & 0.00 & $\# \# \# \# \# \# \#$ & 0.00 \\
\hline 71 & 2000 & 0.5 & 0.00 & \#\#\#\#\#\#\# & 0.00 \\
\hline 72 & 2000 & 1 & 0.00 & 2564206.82 & 1.02 \\
\hline 73 & 2000 & 1 & 0.00 & 4754985.47 & 0.00 \\
\hline 74 & 2000 & 1 & 0.00 & 3248825.15 & $\overline{0.76}$ \\
\hline 75 & 2000 & 3 & 0.00 & 1170074.96 & 0.00 \\
\hline 76 & 2000 & 3 & 0.00 & 1157627.35 & 0.00 \\
\hline 77 & 2000 & 3 & 0.00 & 759303.96 & 2.29 \\
\hline$\overline{78}$ & 2000 & 7 & 0.00 & 2352597.52 & 2.04 \\
\hline 79 & 2000 & 7 & 0.00 & 2452178.37 & 2.29 \\
\hline 80 & 2000 & 7 & 0.00 & $\mid 1966721.74$ & 2.04 \\
\hline 81 & 2000 & 14 & 0.00 & 4033024.32 & 2.55 \\
\hline 82 & 2000 & 14 & 0.00 & 3858757.84 & 1.27 \\
\hline 83 & 2000 & 14 & 0.00 & 4319319.26 & 0.76 \\
\hline 84 & 2000 & 28 & 0.00 & 1282103.41 & 0.76 \\
\hline 85 & 2000 & 28 & 0.00 & 1854693.28 & 2.33 \\
\hline 86 & 2000 & 28 & 0.00 & 2663787.67 & 2.56 \\
\hline$\overline{87}$ & 2000 & 56 & 0.00 & 4916804.34 & 3.38 \\
\hline 88 & 2000 & 56 & 0.00 & 7144925.81 & $\overline{0.96}$ \\
\hline 89 & 2000 & 56 & 0.00 & 6771497.63 & 2.41 \\
\hline 90 & 2000 & 91 & 6.35 & 5564079.85 & 10.85 \\
\hline 91 & 2000 & 91 & 3.17 & $\# \# \# \# \# \# \#$ & 5.55 \\
\hline 92 & 2000 & 91 & 0.00 & $\# \# \# \# \# \# \#$ & 6.03 \\
\hline 93 & 2000 & 182 & 0.00 & 4605614.20 & 1.45 \\
\hline 94 & 2000 & 182 & 0.00 & 6236250.57 & 0.96 \\
\hline
\end{tabular}


NORM. CONC. (g/m2, blank corrected) FOR 18A FILTERED LEACHATES (all tests)

\begin{tabular}{|r|r|r|r|r|r|}
\hline & \multicolumn{1}{|c|}{ A } & \multicolumn{1}{c|}{ B } & \multicolumn{1}{c|}{ AM } & AN & \multicolumn{1}{c|}{ AO } \\
\hline 1 & SA/V & Duration & & & \\
\hline 2 & $(\mathrm{~m}-1)$ & (days) & Y & ZN & \multicolumn{1}{c|}{ ZR } \\
\hline 95 & 2000 & 182 & 6.35 & 9385494.88 & 5.07 \\
\hline 96 & 20000 & 1 & 0.00 & 7675390.24 & 0.00 \\
\hline 97 & 20000 & 1 & 0.00 & 697065.93 & 0.00 \\
\hline 98 & 20000 & 1 & 0.00 & 2889017.14 & 0.00 \\
\hline 99 & 20000 & 3 & 0.00 & $\# \# \# \#$ & 0.00 \\
\hline 100 & 20000 & 3 & 0.00 & $\# \# \# \# \#$ & 8.69 \\
\hline 101 & 20000 & 3 & 0.00 & $\# \# \# \# \#$ & 8.92 \\
\hline 102 & 20000 & 7 & -15.87 & $\# \# \# \# \# \#$ & 3.82 \\
\hline 103 & 20000 & 7 & -15.87 & $\# \# \# \# \# \#$ & 12.74 \\
\hline 104 & 20000 & 7 & -15.87 & $\# \# \# \# \# \#$ & 10.53 \\
\hline 105 & 20000 & 14 & 0.00 & $\# \# \# \# \# \#$ & 10.19 \\
\hline 106 & 20000 & 14 & 0.00 & 5529724.46 & 7.78 \\
\hline 107 & 20000 & 14 & 0.00 & 7966467.80 & 1.27 \\
\hline 108 & 20000 & 28 & 0.00 & $\# \# \# \# \#$ & 6.99 \\
\hline 109 & 20000 & 28 & 0.00 & $\# \# \# \# \# \#$ & 12.81 \\
\hline 110 & 20000 & 28 & 0.00 & $\# \# \# \# \# \#$ & 10.25 \\
\hline
\end{tabular}




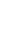


NORM. CONC. (g/m2, blank corrected) FOR 0.45u FILTERED LEACHATES (all tests)

\begin{tabular}{|c|c|c|c|c|c|c|c|}
\hline $\mathrm{SA} / \mathrm{V}$ & Duration & & & & & & \\
\hline$(m-1)$ & (days) & pH final & $\mathrm{pH}$ in & $\mathrm{AL}$ & AS & B & BA \\
\hline 10 & 0.25 & 7.48 & 5.58 & 3.07 & 0.00 & 2.95 & 0.00 \\
\hline 10 & 0.25 & 7.34 & 5.58 & 3.92 & 0.00 & 3.70 & 0.00 \\
\hline 10 & 0.25 & 7.27 & 5.58 & 3.70 & 0.00 & 3.91 & 0.00 \\
\hline 10 & 0.5 & 8.97 & 5.88 & 12.08 & 0.00 & 13.43 & 1.52 \\
\hline 10 & 0.5 & 9.07 & 5.88 & 16.18 & 0.00 & 17.63 & 2.54 \\
\hline 10 & 0.5 & 9.03 & 5.88 & 17.25 & 0.00 & 18.80 & 2.54 \\
\hline 10 & 1 & 9.52 & 5.67 & 29.37 & -132033.49 & 31.55 & 8.68 \\
\hline 10 & 1 & 9.53 & 5.67 & 29.90 & -132033.49 & 32.13 & 8.68 \\
\hline 10 & 1 & 9.49 & 5.67 & 26.92 & -132033.49 & 29.46 & 8.68 \\
\hline 10 & 3 & 9.68 & 5.37 & 62.83 & -132033.49 & 74.31 & 14.89 \\
\hline 10 & 3 & 9.67 & 5.37 & 60.61 & 0.00 & 68.82 & 14.89 \\
\hline 10 & 3 & 9.65 & 5.37 & 59.72 & 0.00 & 67.79 & 16.13 \\
\hline 10 & 7 & 9.55 & 5.74 & 74.42 & 171643.53 & 99.10 & 11.16 \\
\hline 10 & 7 & 9.51 & 5.74 & 74.87 & 66016.74 & 100.82 & 11.16 \\
\hline 10 & 7 & 9.51 & 5.74 & 72.64 & 132033.49 & 97.04 & 11.16 \\
\hline 10 & 14 & 9.72 & 5.74 & 82.44 & -132033.49 & 120.60 & 9.92 \\
\hline 10 & 14 & 9.71 & 5.74 & 82.89 & -132033.49 & 121.29 & 8.68 \\
\hline 10 & 14 & 9.71 & 5.74 & 82.89 & -132033.49 & 121.29 & 9.92 \\
\hline 10 & 28 & 9.34 & 6.01 & 91.09 & 184846.88 & 146.70 & 8.68 \\
\hline 10 & 28 & 9.33 & 6.01 & 91.09 & 39610.05 & 149.78 & 7.44 \\
\hline 10 & 28 & 9.30 & 6.01 & 91.09 & 92423.44 & 149.10 & 8.68 \\
\hline 10 & 56 & 9.43 & 6.01 & 94.34 & 250863.62 & 167.20 & 11.16 \\
\hline 10 & 56 & 9.43 & 6.01 & 93.45 & 250863.62 & 164.80 & 11.16 \\
\hline 10 & 56 & 9.47 & 6.01 & 94.34 & 105626.79 & 166.86 & 9.92 \\
\hline 10 & 91 & 9.31 & 6.01 & 93.58 & 52813.39 & 181.06 & 12.41 \\
\hline 10 & 91 & 9.38 & 6.01 & 93.58 & 39610.05 & 182.77 & 11.16 \\
\hline 10 & 91 & 9.30 & 6.01 & 92.25 & 0.00 & 180.37 & 11.16 \\
\hline 10 & 182 & 9.42 & 6.01 & 94.03 & -132033.49 & 201.02 & 12.41 \\
\hline 10 & 182 & 9.43 & 6.01 & 93.14 & -132033.49 & 196.22 & 9.92 \\
\hline 10 & 182 & 9.48 & 6.01 & 97.59 & 0.00 & 208.22 & 9.92 \\
\hline 10 & 364 & 8.64 & 6.00 & 78.61 & -118830.14 & 226.43 & 11.16 \\
\hline 10 & 364 & 8.76 & 6.00 & 87.08 & 171643.53 & 238.09 & 12.41 \\
\hline 10 & 364 & 8.76 & 6.00 & 87.08 & 171643.53 & 238.09 & 12.41 \\
\hline 530 & 0.25 & 9.88 & 5.58 & 80.75 & 47532.05 & 107.27 & 5.58 \\
\hline 530 & 0.25 & 9.90 & 5.58 & 83.92 & -178245.21 & 113.25 & 7.41 \\
\hline 530 & 0.25 & 9.93 & 5.58 & 90.27 & -180885.88 & 120.72 & 8.48 \\
\hline 530 & 0.5 & 9.95 & 5.88 & 91.30 & -59415.07 & 143.65 & 8.27 \\
\hline 530 & 0.5 & 9.97 & 5.88 & 91.30 & 304997.35 & 147.06 & 7.36 \\
\hline 530 & 0.5 & 9.96 & 5.88 & 89.19 & -108267.46 & 145.61 & 5.43 \\
\hline 530 & 1 & 9.95 & 5.74 & 103.86 & -132033.49 & 198.49 & 28.53 \\
\hline 530 & 1 & 9.95 & 5.74 & 100.80 & -264066.97 & 197.45 & 23.57 \\
\hline 530 & 1 & 9.95 & 5.74 & 99.93 & -132033.49 & 192.96 & 26.05 \\
\hline 530 & 3 & 10.03 & 5.51 & 130.48 & 277270.32 & 304.18 & 26.05 \\
\hline 530 & 3 & 9.91 & 5.51 & 135.71 & 396100.46 & 307.64 & 33.49 \\
\hline 530 & 3 & 10.04 & 5.51 & 137.46 & 343287.06 & 305.91 & 38.46 \\
\hline 530 & 7 & 10.10 & 5.64 & 180.66 & 290473.67 & 418.18 & 55.82 \\
\hline 530 & 7 & 10.06 & 5.64 & 179.79 & 382897.11 & 414.72 & 54.58 \\
\hline
\end{tabular}


NORM. CONC. (g/m2, blank corrected) FOR 0.45u FILTERED LEACHATES (all tests)

\begin{tabular}{|c|c|c|c|c|c|c|c|}
\hline $\mathrm{SA} / \mathrm{V}$ & Duration & & & & & & \\
\hline$(m-1)$ & (days) & pH final & $\mathrm{pH}$ in & $\overline{A L}$ & AS & B & $\mathbf{B A}$ \\
\hline 530 & 7 & 10.10 & 5.64 & 169.75 & 92423.44 & 404.35 & 48.38 \\
\hline 530 & 14 & 9.99 & 5.69 & 206.10 & 356490.41 & 490.81 & 69.47 \\
\hline 530 & 14 & 9.98 & 5.69 & 196.50 & 198050.23 & 487.35 & 60.79 \\
\hline 530 & 14 & 10.00 & 5.69 & 202.61 & 396100.46 & 487.35 & 68.23 \\
\hline 530 & 28 & 10.24 & 5.55 & 252.23 & -66016.74 & 532.83 & 39.08 \\
\hline 530 & 28 & 10.23 & 5.55 & 255.80 & 290473.67 & 532.83 & 39.08 \\
\hline 530 & 28 & 10.15 & 5.55 & 247.77 & -13203.35 & 532.83 & 36.03 \\
\hline 530 & 56 & 10.11 & 5.61 & 230.84 & 382897.11 & 582.82 & 73.19 \\
\hline 530 & 56 & 10.11 & 5.61 & 161.32 & 264066.97 & 579.39 & 44.66 \\
\hline 530 & 56 & 10.07 & 5.61 & 252.68 & 290473.67 & 589.67 & 89.32 \\
\hline 530 & 91 & \begin{tabular}{l|l|}
9.86 \\
\end{tabular} & 5.61 & 224.16 & 382897.11 & 623.69 & 66.99 \\
\hline 530 & 91 & 9.90 & 5.61 & 251.34 & 369693.76 & 627.12 & 76.91 \\
\hline 530 & 91 & 9.88 & 5.61 & 249.56 & 514930.59 & 623.69 & 74.43 \\
\hline 530 & 182 & 9.98 & 5.61 & 258.91 & 290473.67 & 658.39 & 81.88 \\
\hline 530 & 182 & 9.94 & 5.61 & 268.27 & 0.00 & 685.83 & 93.04 \\
\hline 530 & 182 & 9.96 & 5.61 & 263.37 & 118830.14 & 689.26 & 88.08 \\
\hline 2000 & 0.25 & 10.16 & 5.58 & 101.85 & 48852.39 & 211.44 & 2.74 \\
\hline 2000 & 0.25 & 10.13 & 5.58 & 99.39 & 188807.88 & 210.18 & 3.10 \\
\hline 2000 & 0.25 & 10.17 & 5.58 & 103.62 & 34328.71 & 211.94 & 4.72 \\
\hline 2000 & 0.5 & 10.21 & 5.88 & 109.23 & 106947.12 & 286.00 & 3.60 \\
\hline 2000 & 0.5 & 10.18 & 5.88 & 111.34 & 39610.05 & 281.48 & 3.10 \\
\hline 2000 & 0.5 & 10.14 & 5.88 & 103.60 & 182206.21 & 238.68 & 3.35 \\
\hline 2000 & 1 & 10.02 & 5.69 & 121.31 & 237660.27 & 421.85 & 11.16 \\
\hline 2000 & 1 & 10.09 & 5.69 & 122.62 & 224456.93 & 435.68 & 12.41 \\
\hline 2000 & 1 & 10.02 & 5.69 & 127.86 & 224456.93 & 449.51 & 13.65 \\
\hline 2000 & 3 & 10.32 & 5.51 & 168.88 & -158440.18 & 695.14 & 31.01 \\
\hline 2000 & 3 & 10.30 & 5.51 & 163.20 & -158440.18 & 695.14 & 26.05 \\
\hline 2000 & 3 & 10.31 & 5.51 & 163.20 & 158440.18 & 688.23 & 29.77 \\
\hline 2000 & 7 & 10.39 & 5.74 & 247.86 & 528133.94 & 878.38 & 47.14 \\
\hline 2000 & 7 & 10.37 & 5.74 & 234.77 & 541337.29 & 871.46 & 42.18 \\
\hline 2000 & 7 & 10.37 & 5.74 & 242.62 & 514930.59 & 868.01 & 42.18 \\
\hline 2000 & 14 & 10.27 & 5.69 & 284.95 & 567743.99 & 985.43 & 53.34 \\
\hline 2000 & 14 & 10.30 & 5.69 & 276.66 & 514930.59 & 978.51 & 52.10 \\
\hline 2000 & 14 & 10.28 & 5.69 & 283.64 & 303677.02 & 961.22 & 59.55 \\
\hline 2000 & 28 & 10.51 & 5.55 & 315.06 & 514930.59 & 1014.96 & 62.03 \\
\hline 2000 & 28 & 10.56 & 5.55 & 340.91 & 132033.49 & 1093.27 & 36.54 \\
\hline 2000 & 28 & 10.57 & 5.55 & 344.48 & 290473.67 & 1076.05 & 38.57 \\
\hline 2000 & 56 & 10.42 & 5.61 & 351.61 & 594150.69 & 1158.29 & 84.36 \\
\hline 2000 & 56 & 10.45 & 5.61 & 348.49 & 580947.34 & 1168.58 & 81.88 \\
\hline 2000 & 56 & 10.39 & 5.61 & 349.38 & 594150.69 & 1127.43 & 88.08 \\
\hline 2000 & 91 & 10.34 & 5.61 & 450.09 & 633760.73 & 1258.49 & 117.85 \\
\hline 2000 & 91 & 10.23 & 5.61 & 467.92 & 633760.73 & 1279.07 & 119.09 \\
\hline 2000 & 91 & 10.23 & 5.61 & 454.55 & 963844.45 & 1220.77 & 115.37 \\
\hline 2000 & 182 & 10.37 & 5.61 & 543.68 & 396100.46 & 1392.23 & 157.55 \\
\hline 2000 & 182 & 10.36 & 5.61 & 539.22 & 422507.15 & 1436.81 & 164.99 \\
\hline 2000 & 182 & 10.32 & 5.61 & 530.31 & 528133.94 & 1409.38 & 163.75 \\
\hline 20000 & 1 & 10.79 & 5.58 & 152.57 & 122554.54 & 2113.79 & 11.38 \\
\hline
\end{tabular}


NORM. CONC. (g/m2, blank corrected) FOR 0.45u FILTERED LEACHATES (all tests)

\begin{tabular}{|r|r|r|r|r|r|r|r|}
\hline SA/V & \multicolumn{1}{|c|}{ Duration } & & & & & & \\
\hline (m-1) & \multicolumn{1}{|c|}{ (days) } & pH final & \multicolumn{1}{c|}{ pH in } & \multicolumn{1}{c|}{ AL } & \multicolumn{1}{c|}{ AS } & \multicolumn{1}{c|}{ B } & \multicolumn{1}{c|}{ BA } \\
\hline 20000 & 1 & 10.80 & 5.58 & 136.99 & -728824.84 & 2096.71 & 9.54 \\
\hline 20000 & 1 & 10.76 & 5.58 & 155.68 & 1473863.39 & 2329.03 & 10.86 \\
\hline 20000 & 3 & 10.91 & 5.51 & 319.21 & -457628.06 & 3578.62 & 37.22 \\
\hline 20000 & 3 & 10.86 & 5.51 & 284.76 & -555332.84 & 3314.04 & 31.71 \\
\hline 20000 & 3 & 10.90 & 5.51 & 288.67 & -985762.00 & 3301.93 & 37.22 \\
\hline 20000 & 7 & 10.86 & 5.64 & 501.39 & 660167.43 & 3975.32 & 55.82 \\
\hline 20000 & 7 & 10.89 & 5.64 & 518.85 & 1650418.57 & 3923.44 & 62.03 \\
\hline 20000 & 7 & 10.92 & 5.64 & 510.13 & 965428.85 & 3919.05 & 57.28 \\
\hline 20000 & 14 & 10.96 & 5.64 & 663.29 & -660167.43 & 4599.60 & 68.23 \\
\hline 20000 & 14 & 10.98 & 5.64 & 686.33 & 0.00 & 4435.94 & 69.46 \\
\hline 20000 & 14 & 10.85 & 5.64 & 748.38 & 0.00 & 4392.08 & 80.64 \\
\hline 20000 & 28 & 11.21 & 5.55 & 21.61 & 132033.49 & 5179.49 & 0.00 \\
\hline 20000 & 28 & 11.20 & 5.55 & 23.40 & 0.00 & 5179.49 & 0.00 \\
\hline 20000 & 28 & 11.18 & 5.55 & 24.47 & 0.00 & 5034.85 & -2.54 \\
\hline
\end{tabular}


NORM. CONC. (g/m2, blank corrected) FOR 0.45u FILTERED LEACHATES (all tests)

\begin{tabular}{|c|c|c|c|c|c|c|c|}
\hline $\mathrm{SA} / \mathrm{V}$ & Duration & & & & & & \\
\hline$(m-1)$ & (days) & $\mathrm{BE}$ & $\overline{C A}$ & $C D$ & $\mathrm{CE}$ & $\mathrm{CO}$ & $\mathrm{CR}$ \\
\hline 10 & 0.25 & 0.00 & 0.87 & 0.00 & 3.20 & 0.00 & 0.00 \\
\hline 10 & 0.25 & 0.00 & 1.51 & 0.00 & 3.20 & 0.00 & 0.00 \\
\hline 10 & 0.25 & 0.00 & 1.65 & -11423.49 & 0.00 & 0.00 & 0.00 \\
\hline 10 & 0.5 & 0.00 & 10.48 & -22846.98 & 0.00 & 0.00 & 0.00 \\
\hline 10 & 0.5 & 0.00 & 14.14 & -22846.98 & 0.00 & 0.00 & 0.00 \\
\hline 10 & 0.5 & 0.00 & 14.63 & -11423.49 & 0.00 & 0.00 & 0.00 \\
\hline 10 & 1 & 0.00 & 23.17 & 11423.49 & 0.00 & 0.00 & 0.23 \\
\hline 10 & 1 & 0.00 & 23.76 & 11423.49 & 0.00 & 0.00 & 0.00 \\
\hline 10 & 1 & 0.00 & 21.18 & 0.00 & 0.00 & 0.00 & 0.00 \\
\hline 10 & 3 & 0.00 & 53.93 & 0.00 & 0.00 & 0.00 & -0.23 \\
\hline 10 & 3 & 0.00 & 51.98 & 0.00 & 0.00 & 0.00 & 0.12 \\
\hline 10 & 3 & 0.00 & 51.01 & 0.00 & 0.00 & 0.00 & 0.00 \\
\hline 10 & 7 & 0.00 & 62.67 & 0.00 & 0.00 & 0.00 & 0.12 \\
\hline 10 & 7 & 0.00 & 63.16 & 0.00 & 0.00 & 0.00 & 0.23 \\
\hline 10 & 7 & 0.00 & 61.70 & 0.00 & 0.00 & 0.00 & 0.12 \\
\hline 10 & 14 & 0.00 & 65.54 & 0.00 & 0.00 & 0.00 & 0.35 \\
\hline 10 & 14 & 0.00 & 65.54 & 0.00 & 0.00 & 0.00 & 0.12 \\
\hline 10 & 14 & 0.00 & 66.02 & 0.00 & 0.00 & 0.00 & 0.12 \\
\hline 10 & 28 & 0.00 & 72.19 & 0.00 & 0.00 & -14072.41 & 0.35 \\
\hline 10 & 28 & 0.00 & 72.19 & 0.00 & 0.00 & -14072.41 & 0.46 \\
\hline 10 & 28 & 0.00 & 72.68 & -11423.49 & 0.00 & -14072.41 & 0.12 \\
\hline 10 & 56 & 0.00 & 74.72 & 11423.49 & 0.00 & 14072.41 & 0.69 \\
\hline 10 & 56 & 0.00 & 74.24 & 0.00 & 0.00 & 0.00 & 0.92 \\
\hline 10 & 56 & 0.00 & 73.26 & 0.00 & 0.00 & 0.00 & 0.46 \\
\hline 10 & 91 & 0.00 & 69.96 & 0.00 & 0.00 & 0.00 & 0.46 \\
\hline 10 & 91 & 0.00 & 73.85 & 0.00 & 0.00 & 0.00 & 0.69 \\
\hline 10 & 91 & 0.00 & 69.96 & 0.00 & 0.00 & 0.00 & 0.58 \\
\hline 10 & 182 & 0.00 & 86.48 & 0.00 & 0.00 & 0.00 & 1.61 \\
\hline 10 & 182 & 0.00 & 85.51 & 0.00 & 0.00 & 14072.41 & 1.15 \\
\hline 10 & 182 & 0.00 & 88.42 & 0.00 & 0.00 & 0.00 & 0.92 \\
\hline 10 & 364 & 0.00 & 89.44 & 34270.46 & 3.20 & 0.00 & 0.81 \\
\hline 10 & 364 & 0.00 & 88.47 & 0.00 & 8.01 & 0.00 & 1.27 \\
\hline 10 & 364 & 0.00 & 88.47 & 0.00 & 8.01 & 0.00 & 1.27 \\
\hline 530 & 0.25 & 0.00 & 44.78 & -23989.32 & 0.00 & 0.00 & 1.05 \\
\hline 530 & 0.25 & 0.00 & 47.39 & -20562.28 & 0.00 & 0.00 & 1.40 \\
\hline 530 & 0.25 & 0.00 & 47.85 & -14850.53 & 0.00 & 0.00 & 2.01 \\
\hline 530 & 0.5 & 2775.37 & 47.24 & 0.00 & 13.40 & 0.00 & 3.67 \\
\hline 530 & 0.5 & 2775.37 & 45.09 & 0.00 & 6.48 & 0.00 & 2.89 \\
\hline 530 & 0.5 & 0.00 & 41.86 & -4569.40 & 0.00 & 0.00 & 1.84 \\
\hline 530 & 1 & 0.00 & 49.05 & 0.00 & 12.28 & 14072.41 & 7.54 \\
\hline 530 & 1 & 0.00 & 45.89 & 0.00 & 5.67 & 14072.41 & 6.61 \\
\hline 530 & 1 & 0.00 & 47.74 & 0.00 & 7.09 & 14072.41 & 6.73 \\
\hline 530 & 3 & 0.00 & 38.21 & 0.00 & 16.54 & 56289.63 & 4.76 \\
\hline 530 & 3 & 0.00 & 45.04 & 0.00 & 21.26 & 84434.44 & 6.38 \\
\hline 530 & 3 & 0.00 & 49.90 & 0.00 & 28.82 & 84434.44 & 7.31 \\
\hline 530 & 7 & 0.00 & 72.77 & 0.00 & 20.79 & 42217.22 & 7.77 \\
\hline 530 & 7 & 0.00 & 72.27 & 0.00 & 23.15 & 42217.22 & 7.66 \\
\hline
\end{tabular}


NORM. CONC. (g/m2, blank corrected) FOR 0.45u FILTERED LEACHATES (all tests)

\begin{tabular}{|c|c|c|c|c|c|c|c|}
\hline $\mathrm{SA} / \mathrm{V}$ & Duration & & & & & & \\
\hline$(m-1)$ & (days) & BE & CA & $C D$ & $\mathrm{CE}$ & $\mathrm{CO}$ & CR \\
\hline 530 & 7 & 0.00 & 66.75 & 0.00 & 17.48 & 56289.63 & 7.19 \\
\hline 530 & 14 & 0.00 & 99.95 & 5734590.75 & 34.49 & 42217.22 & 9.28 \\
\hline 530 & 14 & 0.00 & 90.42 & 6911209.96 & 25.04 & 14072.41 & 7.66 \\
\hline 530 & 14 & 0.00 & 98.45 & \#\#\#\#\#\#\# & 32.13 & 28144.81 & 8.93 \\
\hline 530 & 28 & 0.00 & 136.46 & 0.00 & 55.84 & 14072.41 & 13.56 \\
\hline 530 & 28 & 0.00 & 133.99 & 0.00 & 59.74 & 14072.41 & 16.02 \\
\hline 530 & 28 & 0.00 & 128.55 & 22846.98 & 42.43 & 0.00 & 14.73 \\
\hline 530 & 56 & 0.00 & 120.00 & 11423.49 & 38.45 & 70362.04 & 12.54 \\
\hline 530 & 56 & 0.00 & 74.82 & 22846.98 & 28.84 & 42217.22 & 10.47 \\
\hline 530 & 56 & 0.00 & 134.09 & 22846.98 & 61.42 & 84434.44 & 16.57 \\
\hline 530 & 91 & 0.00 & 113.10 & 0.00 & 48.07 & 98506.85 & 14.96 \\
\hline 530 & 91 & 0.00 & 130.59 & 0.00 & 46.46 & 70362.04 & 15.65 \\
\hline 530 & 91 & 0.00 & 127.68 & 0.00 & 41.66 & 84434.44 & 15.31 \\
\hline 530 & 182 & 0.00 & 144.63 & 11423.49 & 37.39 & 28144.81 & 19.45 \\
\hline 530 & 182 & 0.00 & 159.69 & 22846.98 & 51.27 & 14072.41 & 22.21 \\
\hline 530 & 182 & 0.00 & 152.89 & 0.00 & 41.66 & 14072.41 & 20.72 \\
\hline 2000 & 0.25 & 2775.37 & 12.46 & 4569.40 & 0.00 & 0.00 & 1.31 \\
\hline 2000 & 0.25 & 0.00 & 13.39 & 19419.93 & 0.00 & 0.00 & 0.96 \\
\hline 2000 & 0.25 & 2775.37 & 17.08 & 33128.11 & 0.00 & 0.00 & 1.75 \\
\hline 2000 & 0.5 & 0.00 & 13.24 & -7996.44 & 0.00 & 0.00 & 1.84 \\
\hline 2000 & 0.5 & 5550.74 & 11.54 & -22846.98 & 0.00 & 0.00 & 1.58 \\
\hline 2000 & 0.5 & 5550.74 & 13.85 & -9138.79 & 0.00 & 0.00 & 1.58 \\
\hline 2000 & 1 & 0.00 & 15.60 & 0.00 & 12.76 & 84434.44 & 4.29 \\
\hline 2000 & 1 & 0.00 & 15.95 & 11423.49 & 15.12 & 84434.44 & 4.18 \\
\hline 2000 & 1 & 0.00 & 18.66 & 11423.49 & 16.54 & 98506.85 & 5.10 \\
\hline 2000 & 3 & 0.00 & 49.00 & 0.00 & 16.54 & 28144.81 & 9.74 \\
\hline 2000 & 3 & 0.00 & 39.97 & 0.00 & 8.50 & 28144.81 & 9.05 \\
\hline 2000 & 3 & 0.00 & 46.19 & 0.00 & 15.12 & 56289.63 & 9.63 \\
\hline 2000 & 7 & 0.00 & 88.32 & 0.00 & 59.06 & 253303.33 & 18.44 \\
\hline 2000 & 7 & 0.00 & 77.28 & 0.00 & 50.08 & 211086.11 & 16.47 \\
\hline 2000 & 7 & 0.00 & 81.29 & 0.00 & 46.77 & 225158.52 & 16.59 \\
\hline 2000 & 14 & 0.00 & 100.65 & \#\#\#\#\#\#\# & 67.09 & 197013.70 & 20.07 \\
\hline 2000 & 14 & 0.00 & 98.14 & $\# \# \# \# \# \# \#$ & 63.31 & 197013.70 & 18.91 \\
\hline 2000 & 14 & 0.00 & 106.67 & \#\#\#\#\#\#\# & 61.89 & 168868.89 & 21.11 \\
\hline 2000 & 28 & 0.00 & 122.87 & 22846.98 & 43.47 & 56289.63 & 22.39 \\
\hline 2000 & 28 & 0.00 & 134.48 & 22846.98 & 83.19 & 126651.67 & 23.39 \\
\hline 2000 & 28 & 0.00 & 148.33 & 11423.49 & 96.59 & 140724.07 & 23.50 \\
\hline 2000 & 56 & 0.00 & 149.10 & 22846.98 & 127.64 & 267375.74 & 35.91 \\
\hline 2000 & 56 & 0.00 & 145.22 & 34270.46 & 127.11 & 295520.56 & 35.22 \\
\hline 2000 & 56 & 0.00 & 158.33 & 45693.95 & 139.39 & 281448.15 & 36.02 \\
\hline 2000 & 91 & 0.00 & 207.16 & 0.00 & 179.45 & 295520.56 & 47.99 \\
\hline 2000 & 91 & 0.00 & 222.71 & 0.00 & 184.79 & 309592.96 & 49.95 \\
\hline 2000 & 91 & 0.00 & 215.42 & 0.00 & 176.25 & 365882.59 & 48.11 \\
\hline 2000 & 182 & 0.00 & 281.39 & 34270.46 & 263.83 & 295520.56 & 71.81 \\
\hline 2000 & 182 & 0.00 & 299.37 & 22846.98 & 282.53 & 253303.33 & 68.59 \\
\hline 2000 & 182 & 0.00 & 302.77 & 45693.95 & 278.79 & 211086.11 & 66.86 \\
\hline 20000 & 1 & 11106.48 & 44.34 & 86999.45 & 0.00 & 0.00 & 9.45 \\
\hline
\end{tabular}


NORM. CONC. (g/m2, blank corrected) FOR 0.45u FILTERED LEACHATES (all tests)

\begin{tabular}{|r|r|r|r|r|r|r|r|}
\hline SA/V & Duration & & & & & & \\
\hline (m-1) & \multicolumn{1}{|c|}{ (days) } & \multicolumn{1}{c|}{ BE } & \multicolumn{1}{c|}{ CA } & \multicolumn{1}{c|}{ CD } & \multicolumn{1}{c|}{ CE } & \multicolumn{1}{c|}{ CO } & \multicolumn{1}{c|}{ CR } \\
\hline 20000 & 1 & 22202.97 & 40.03 & 77679.72 & 0.00 & 0.00 & 10.15 \\
\hline 20000 & 1 & 12750.06 & 46.34 & 104547.76 & 0.00 & 0.00 & 14.07 \\
\hline 20000 & 3 & 0.00 & 104.08 & 0.00 & 40.16 & 1266798.11 & 46.40 \\
\hline 20000 & 3 & 0.00 & 92.60 & 0.00 & -20.13 & 1188836.98 & 42.50 \\
\hline 20000 & 3 & 0.00 & 97.56 & 0.00 & 7.09 & 1266798.11 & 46.40 \\
\hline 20000 & 7 & 0.00 & 149.20 & 0.00 & 146.46 & 1407240.74 & 84.68 \\
\hline 20000 & 7 & 0.00 & 149.20 & 0.00 & 167.72 & 1688688.89 & 87.00 \\
\hline 20000 & 7 & 0.00 & 146.07 & 0.00 & 143.73 & 1595529.55 & 82.19 \\
\hline 20000 & 14 & 0.00 & 152.21 & 114234.88 & 151.18 & 1618326.85 & 127.60 \\
\hline 20000 & 14 & 0.00 & 157.20 & 58145.55 & 163.53 & 1504199.63 & 131.08 \\
\hline 20000 & 14 & 0.00 & 183.30 & 114234.88 & 219.69 & 1829412.96 & 144.42 \\
\hline 20000 & 28 & 0.00 & 19.78 & 0.00 & 0.00 & 70362.04 & 56.71 \\
\hline 20000 & 28 & 0.00 & 16.07 & 0.00 & 0.00 & 0.00 & 57.29 \\
\hline 20000 & 28 & 0.00 & 0.00 & 0.00 & 0.00 & 309592.96 & 56.59 \\
\hline
\end{tabular}


NORM. CONC. ( $\mathrm{g} / \mathrm{m} 2$, blank corrected) FOR 0.45u FILTERED LEACHATES (all tests)

\begin{tabular}{|c|c|c|c|c|c|c|c|}
\hline SA/V & Duration & & & & & & \\
\hline$(m-1)$ & (days) & $\mathrm{CS}$ & $\mathrm{CU}$ & DY & EU & FE & GD \\
\hline 10 & 0.25 & 0.00 & 4.17 & 11476.92 & 0.00 & 0.00 & 0.00 \\
\hline 10 & 0.25 & 0.96 & 3.13 & 11476.92 & 0.00 & 0.00 & 0.00 \\
\hline 10 & 0.25 & 0.96 & 2.09 & 0.00 & 0.00 & 0.00 & 0.00 \\
\hline 10 & 0.5 & 11.78 & 2.38 & 0.00 & 0.00 & 0.00 & 0.00 \\
\hline 10 & 0.5 & 18.85 & 0.00 & 0.00 & 0.00 & 0.00 & 0.00 \\
\hline 10 & 0.5 & 18.85 & 0.00 & 0.00 & 0.00 & 0.00 & 0.00 \\
\hline 10 & 1 & 33.73 & -3.13 & 0.00 & 0.00 & 0.00 & 0.00 \\
\hline 10 & 1 & 38.55 & -3.13 & 0.00 & 0.00 & 0.00 & 0.00 \\
\hline 10 & 1 & 32.77 & -3.13 & 0.00 & 0.00 & 0.00 & 0.00 \\
\hline 10 & 3 & 110.84 & 1.04 & 0.00 & 0.00 & 0.00 & -115262.32 \\
\hline 10 & 3 & 101.20 & 2.09 & 0.00 & 0.00 & 0.00 & -115262.32 \\
\hline 10 & 3 & 101.20 & 0.00 & 0.00 & 0.00 & 0.00 & 0.00 \\
\hline 10 & 7 & 149.39 & 1.04 & 0.00 & 0.00 & 0.00 & -115262.32 \\
\hline 10 & 7 & 130.11 & 3.13 & 0.00 & 0.00 & 0.00 & -115262.32 \\
\hline 10 & 7 & 130.11 & 2.09 & 0.00 & 0.00 & 0.00 & -115262.32 \\
\hline 10 & 14 & 139.75 & 2.09 & 0.00 & 0.00 & 0.00 & 23052.46 \\
\hline 10 & 14 & 149.39 & 2.09 & 0.00 & 0.00 & 0.00 & -207472.18 \\
\hline 10 & 14 & 139.75 & 1.04 & 0.00 & 0.00 & 0.00 & -92209.86 \\
\hline 10 & 28 & 159.03 & 2.09 & 0.00 & 0.00 & 0.00 & -115262.32 \\
\hline 10 & 28 & 159.03 & 2.09 & 0.00 & 0.00 & 0.00 & -115262.32 \\
\hline 10 & 28 & 168.67 & 2.09 & 0.00 & 0.00 & 0.00 & -115262.32 \\
\hline 10 & 56 & 187.94 & 5.22 & 0.00 & 0.00 & 0.00 & -115262.32 \\
\hline 10 & 56 & 187.94 & 4.17 & 0.00 & 0.00 & 0.00 & -230524.64 \\
\hline 10 & 56 & 187.94 & 3.13 & 0.00 & 0.00 & 0.00 & -115262.32 \\
\hline 10 & 91 & 216.86 & 0.00 & 0.00 & 0.00 & 0.00 & -230524.64 \\
\hline 10 & 91 & 216.86 & 2.09 & 0.00 & 0.00 & 0.00 & -230524.64 \\
\hline 10 & 91 & 216.86 & 0.00 & 0.00 & 0.00 & 0.00 & -230524.64 \\
\hline 10 & 182 & 236.13 & 0.00 & 0.00 & 0.00 & 0.00 & -230524.64 \\
\hline 10 & 182 & 216.86 & 0.00 & 0.00 & 0.00 & 0.00 & 0.00 \\
\hline 10 & 182 & 236.13 & 0.00 & 0.00 & 0.00 & 0.00 & 23052.46 \\
\hline 10 & 364 & 265.05 & 10.43 & -11476.92 & 0.00 & 0.00 & -103736.09 \\
\hline 10 & 364 & 284.32 & 12.52 & 0.00 & 0.00 & 0.00 & -230524.64 \\
\hline 10 & 364 & 284.32 & 12.52 & 0.00 & 0.00 & 0.00 & -230524.64 \\
\hline 530 & 0.25 & 182.59 & 15.92 & 0.00 & 0.00 & 0.00 & 0.00 \\
\hline 530 & 0.25 & 194.37 & 21.94 & 0.00 & 0.00 & 0.00 & 0.00 \\
\hline 530 & 0.25 & 206.15 & 22.29 & 0.00 & 0.00 & 0.00 & 0.00 \\
\hline 530 & 0.5 & 229.71 & 33.68 & 0.00 & 0.00 & 0.00 & 0.00 \\
\hline 530 & 0.5 & 217.93 & 29.69 & 0.00 & 0.00 & 0.00 & 0.00 \\
\hline 530 & 0.5 & 217.93 & 22.53 & 0.00 & 0.00 & 0.00 & 0.00 \\
\hline 530 & 1 & 162.82 & 83.45 & 0.00 & 0.00 & 0.00 & -230524.64 \\
\hline 530 & 1 & 162.82 & 74.07 & 0.00 & 0.00 & 0.00 & -230524.64 \\
\hline 530 & 1 & 155.24 & 76.15 & 0.00 & 0.00 & 0.00 & -230524.64 \\
\hline 530 & 3. & 230.97 & 81.37 & 0.00 & 0.00 & 0.00 & -230524.64 \\
\hline 530 & 3 & 223.40 & 97.02 & 0.00 & 0.00 & 0.00 & -230524.64 \\
\hline 530 & 3 & 230.97 & 107.45 & 0.00 & 11579.36 & 11579.36 & 0.00 \\
\hline 530 & 7 & 299.13 & 152.30 & 0.00 & 0.00 & 0.00 & -461049.28 \\
\hline 530 & 7 & 314.27 & 156.48 & 0.00 & 0.00 & 0.00 & -461049.28 \\
\hline
\end{tabular}


NORM. CONC. (g/m2, blank corrected) FOR 0.45u FILTERED LEACHATES (all tests)

\begin{tabular}{|c|c|c|c|c|c|c|c|}
\hline SA/V & Duration & & & & & & \\
\hline$(m-1)$ & (days) & $C S$ & $\mathrm{CU}$ & DY & $\mathrm{EU}$ & FE & GD \\
\hline 530 & 7 & 283.98 & 136.66 & 0.00 & 0.00 & 0.00 & -345786.96 \\
\hline 530 & 14 & 314.27 & 203.42 & -11476.92 & 11579.36 & 11579.36 & 11526.23 \\
\hline 530 & 14 & 306.70 & 177.34 & -11476.92 & 11579.36 & 11579.36 & -92209.86 \\
\hline 530 & 14 & 283.98 & 197.16 & -11476.92 & 11579.36 & 11579.36 & -69157.39 \\
\hline 530 & 28 & 488.87 & 157.97 & 0.00 & 0.00 & 0.00 & -576311.61 \\
\hline 530 & 28 & 465.31 & 160.35 & 0.00 & 0.00 & 0.00 & -806836.25 \\
\hline 530 & 28 & 453.53 & 149.62 & 0.00 & 0.00 & 0.00 & -691573.93 \\
\hline 530 & 56 & 371.07 & 261.84 & 0.00 & 0.00 & 0.00 & -345786.96 \\
\hline 530 & 56 & 274.69 & 151.26 & 0.00 & 0.00 & 0.00 & -345786.96 \\
\hline 530 & 56 & 399.98 & 304.61 & 0.00 & 11579.36 & 11579.36 & -345786.96 \\
\hline 530 & 91 & 399.98 & 242.02 & 11476.92 & 11579.36 & 11579.36 & -115262.32 \\
\hline 530 & 91 & 419.26 & 287.92 & 0.00 & 11579.36 & 11579.36 & -345786.96 \\
\hline 530 & 91 & 438.53 & 285.83 & 0.00 & 11579.36 & 11579.36 & -461049.28 \\
\hline 530 & 182 & 506.00 & 309.82 & 0.00 & 0.00 & 0.00 & -461049.28 \\
\hline 530 & 182 & 457.81 & 318.17 & 0.00 & 0.00 & 0.00 & -461049.28 \\
\hline 530 & 182 & 448.17 & 319.21 & 0.00 & 0.00 & 0.00 & -576311.61 \\
\hline 2000 & 0.25 & 288.61 & 17.70 & 0.00 & 0.00 & 0.00 & 0.00 \\
\hline 2000 & 0.25 & 265.05 & 19.31 & 0.00 & 0.00 & 0.00 & 0.00 \\
\hline 2000 & 0.25 & 323.95 & 28.08 & 0.00 & 0.00 & 0.00 & 0.00 \\
\hline 2000 & 0.5 & 265.05 & 27.06 & 0.00 & 0.00 & 0.00 & 0.00 \\
\hline 2000 & 0.5 & 253.27 & 23.49 & 0.00 & 0.00 & 0.00 & 0.00 \\
\hline 2000 & 0.5 & 276.83 & 19.91 & 0.00 & 0.00 & 0.00 & 0.00 \\
\hline 2000 & 1 & 155.24 & 67.81 & 0.00 & 0.00 & 0.00 & -230524.64 \\
\hline 2000 & 1 & 147.67 & 69.89 & 0.00 & 0.00 & 0.00 & -230524.64 \\
\hline 2000 & 1 & 162.82 & 81.37 & 0.00 & 0.00 & 0.00 & -230524.64 \\
\hline 2000 & 3 & 155.24 & 190.90 & -11476.92 & 0.00 & 0.00 & -668521.46 \\
\hline 2000 & 3 & 200.68 & 167.95 & -11476.92 & 0.00 & 0.00 & -553259.14 \\
\hline 2000 & 3 & 155.24 & 184.64 & -11476.92 & 0.00 & 0.00 & -553259.14 \\
\hline 2000 & 7 & 253.69 & 441.26 & 0.00 & 0.00 & 0.00 & -345786.96 \\
\hline 2000 & 7 & 246.12 & 389.10 & 0.00 & 0.00 & 0.00 & -461049.28 \\
\hline 2000 & 7 & 253.69 & 405.80 & 0.00 & 0.00 & 0.00 & -345786.96 \\
\hline 2000 & 14 & 336.99 & 472.56 & 11476.92 & 11579.36 & 11579.36 & -437996.82 \\
\hline 2000 & 14 & 283.98 & 467.34 & 22953.85 & 11579.36 & 11579.36 & -207472.18 \\
\hline 2000 & 14 & 299.13 & 486.12 & 0.00 & 0.00 & 0.00 & -668521.46 \\
\hline 2000 & 28 & 268.83 & 564.36 & 0.00 & 0.00 & 0.00 & $\# \# \# \# \# \# \#$ \\
\hline 2000 & 28 & 488.87 & 395.81 & 0.00 & 0.00 & 0.00 & $\# \# \# \# \# \# \#$ \\
\hline 2000 & 28 & 453.53 & 406.54 & 0.00 & 0.00 & 0.00 & $\# \# \# \# \# \#$ \\
\hline 2000 & 56 & 419.26 & 693.71 & 0.00 & 11579.36 & 11579.36 & -806836.25 \\
\hline 2000 & 56 & 419.26 & 697.88 & 11476.92 & 11579.36 & 11579.36 & -576311.61 \\
\hline 2000 & 56 & 428.90 & 707.27 & 11476.92 & 23158.73 & 23158.73 & -576311.61 \\
\hline 2000 & 91 & 612.02 & 881.48 & 0.00 & 23158.73 & 23158.73 & -576311.61 \\
\hline 2000 & 91 & 631.30 & 905.48 & 11476.92 & 23158.73 & 23158.73 & -576311.61 \\
\hline 2000 & 91 & 640.94 & 854.36 & 11476.92 & 23158.73 & 23158.73 & -461049.28 \\
\hline 2000 & 182 & 785.51 & 964.94 & 0.00 & 23158.73 & 23158.73 & -922098.57 \\
\hline 2000 & 182 & 862.61 & 909.65 & 0.00 & 11579.36 & 11579.36 & -922098.57 \\
\hline 2000 & 182 & 852.97 & 923.21 & 0.00 & 11579.36 & 11579.36 & $\# \# \# \# \# \# \#$ \\
\hline 20000 & 1 & 259.29 & 489.51 & 0.00 & 0.00 & 0.00 & 0.00 \\
\hline
\end{tabular}


NORM. CONC. (g/m2, blank corrected) FOR 0.45u FILTERED LEACHATES (all tests)

\begin{tabular}{|r|r|r|r|r|r|r|r|}
\hline SA/V & Duration & & & & & & \\
\hline (m-1) & (days) & CS & CU & DY & EU & FE & GD \\
\hline 20000 & 1 & 273.29 & 454.23 & 0.00 & 0.00 & 0.00 & 0.00 \\
\hline 20000 & 1 & 306.55 & 509.95 & 0.00 & 0.00 & 0.00 & 0.00 \\
\hline 20000 & 3 & 337.07 & 2420.19 & 0.00 & 0.00 & 0.00 & $\# \# \# \# \#$ \\
\hline 20000 & 3 & 336.01 & 2372.30 & 0.00 & 0.00 & 0.00 & $\# \# \# \# \#$ \\
\hline 20000 & 3 & 280.27 & 2284.57 & 0.00 & 0.00 & 0.00 & $\# \# \# \# \#$ \\
\hline 20000 & 7 & 435.44 & 4136.19 & -172153.85 & 0.00 & 0.00 & $\# \# \# \# \#$ \\
\hline 20000 & 7 & 549.03 & 4308.31 & -114769.23 & 57896.81 & 57896.81 & $\# \# \# \# \#$ \\
\hline 20000 & 7 & 524.95 & 4217.24 & -172153.85 & 0.00 & 0.00 & $\# \# \# \# \#$ \\
\hline 20000 & 14 & 435.44 & 5002.02 & 0.00 & 0.00 & 0.00 & $\# \# \# \# \#$ \\
\hline 20000 & 14 & 405.07 & 5107.99 & 0.00 & 0.00 & 0.00 & $\# \# \# \# \#$ \\
\hline 20000 & 14 & 397.57 & 5179.36 & 0.00 & 0.00 & 0.00 & $\# \# \# \# \#$ \\
\hline 20000 & 28 & 164.92 & 8.94 & 0.00 & 0.00 & 0.00 & $\# \# \# \# \#$ \\
\hline 20000 & 28 & 194.37 & 5.96 & 57384.62 & 0.00 & 0.00 & $\# \# \# \# \#$ \\
\hline 20000 & 28 & 139.00 & 6.56 & 195107.69 & 0.00 & 0.00 & 253577.11 \\
\hline
\end{tabular}


NORM. CONC. (g/m2, blank corrected) FOR 0.45u FILTERED LEACHATES (all tests)

\begin{tabular}{|c|c|c|c|c|c|c|c|}
\hline SA/V & Duration & & & & & & \\
\hline$(m-1)$ & (days) & $\mathbf{K}$ & LA & $\mathbf{L I}$ & MG & $\mathbf{M N}$ & $\mathrm{MO}$ \\
\hline 10 & 0.25 & 84321.52 & 0.41 & 3.17 & 0.43 & 0.93 & 2.68 \\
\hline 10 & 0.25 & 0.00 & 0.21 & 4.02 & 1.94 & 0.93 & 2.68 \\
\hline 10 & 0.25 & 0.00 & 0.00 & 3.72 & 0.00 & 0.93 & 2.68 \\
\hline 10 & 0.5 & 0.00 & 0.00 & 12.15 & 7.02 & 0.99 & 0.00 \\
\hline 10 & 0.5 & 0.00 & 0.00 & 16.14 & 8.29 & 0.99 & 15.58 \\
\hline 10 & 0.5 & 0.00 & 0.00 & 17.07 & 9.99 & 0.99 & 16.73 \\
\hline 10 & 1 & 0.00 & 0.00 & 29.94 & 20.89 & 1.86 & 27.33 \\
\hline 10 & 1 & 0.00 & 0.00 & 29.94 & 21.96 & 0.93 & 27.33 \\
\hline 10 & 1 & 0.00 & 0.00 & 29.45 & 19.81 & 0.93 & 23.58 \\
\hline 10 & 3 & 0.00 & 0.00 & 74.39 & 35.10 & 2.79 & 71.80 \\
\hline 10 & 3 & 0.00 & 0.00 & 67.68 & 42.42 & 2.79 & 66.98 \\
\hline 10 & 3. & 0.00 & 0.00 & 67.68 & 43.07 & 1.86 & 64.83 \\
\hline 10 & 7 & 0.00 & 0.00 & 99.39 & 8.18 & 2.79 & 93.77 \\
\hline 10 & 7 & 0.00 & 0.00 & 101.22 & 8.40 & 1.86 & 95.38 \\
\hline 10 & 7 & 0.00 & 0.00 & 97.56 & 6.68 & 1.86 & 90.02 \\
\hline 10 & 14 & 0.00 & 0.00 & 118.90 & 4.74 & 2.79 & 115.74 \\
\hline 10 & 14 & 0.00 & 0.00 & 121.34 & 2.58 & 2.79 & 117.35 \\
\hline 10 & 14 & 0.00 & 0.00 & 123.17 & 1.29 & 2.79 & 117.88 \\
\hline 10 & 28 & 0.00 & 0.00 & 147.56 & 0.65 & 1.86 & 138.24 \\
\hline 10 & 28 & 0.00 & 0.00 & 151.83 & 4.95 & 2.79 & 141.46 \\
\hline 10 & 28 & 0.00 & 0.00 & 151.22 & 3.23 & 2.79 & 138.78 \\
\hline 10 & 56 & 0.00 & 0.00 & 166.46 & 8.40 & 4.65 & 160.75 \\
\hline 10 & 56 & 0.00 & 0.00 & 166.46 & 7.32 & 4.65 & 157.53 \\
\hline 10 & 56 & 0.00 & 0.00 & 168.29 & 4.95 & 3.72 & 158.60 \\
\hline 10 & 91 & 0.00 & -2.06 & 180.36 & 3.23 & 6.52 & 172.00 \\
\hline 10 & 91 & 0.00 & -2.06 & 179.75 & 4.74 & 5.59 & 174.14 \\
\hline 10 & 91 & 0.00 & -2.06 & 179.14 & 0.22 & 4.65 & 170.39 \\
\hline 10 & 182 & 1047996.01 & 0.00 & 198.53 & 11.84 & 7.45 & 190.22 \\
\hline 10 & 182 & 2144175.75 & 0.62 & 194.27 & 16.58 & 8.38 & 187.54 \\
\hline 10 & 182 & -843215.18 & 0.82 & 205.85 & 11.41 & 7.45 & 197.18 \\
\hline 10 & 364 & 0.00 & 0.82 & 227.44 & 13.57 & 8.38 & 223.44 \\
\hline 10 & 364 & 0.00 & 1.23 & 241.46 & 18.09 & 10.24 & 231.48 \\
\hline 10 & 364 & 0.00 & 1.23 & 241.46 & 18.09 & 10.24 & 231.48 \\
\hline 530 & 0.25 & 0.00 & 10.32 & 122.02 & 37.61 & 15.53 & 106.87 \\
\hline 530 & 0.25 & 0.00 & 16.82 & 127.83 & 40.75 & 21.46 & 115.93 \\
\hline 530 & 0.25 & 0.00 & 17.95 & 136.40 & 39.18 & 23.44 & 119.62 \\
\hline 530 & 0.5 & 0.00 & 29.59 & 164.00 & 42.32 & 35.51 & 148.93 \\
\hline 530 & 0.5 & 0.00 & 25.28 & 167.35 & 36.05 & 31.45 & 152.11 \\
\hline 530 & 0.5 & 0.00 & 16.80 & 168.19 & 34.48 & 23.93 & 147.32 \\
\hline 530 & 1 & 0.00 & 38.12 & 217.04 & 56.85 & 63.30 & 203.44 \\
\hline 530 & 1 & 0.00 & 31.41 & 216.44 & 46.19 & 53.41 & 202.84 \\
\hline 530 & 1 & 0.00 & 33.30 & 209.23 & 48.36 & 56.38 & 199.24 \\
\hline 530 & 3 & 0.00 & 22.41 & 321.00 & 109.55 & 80.11 & 325.27 \\
\hline 530 & 3 & 0.00 & 29.53 & 323.40 & 126.73 & 96.93 & 330.07 \\
\hline 530 & 3 & 0.00 & 35.39 & 322.20 & 139.16 & 104.84 & 327.07 \\
\hline 530 & 7 & 0.00 & 33.72 & 451.64 & 219.70 & 175.06 & 448.29 \\
\hline 530 & 7 & 0.00 & 33.30 & 445.03 & 219.70 & 175.06 & 441.69 \\
\hline
\end{tabular}


NORM. CONC. (g/m2, blank corrected) FOR 0.45u FILTERED LEACHATES (all tests)

\begin{tabular}{|c|c|c|c|c|c|c|c|}
\hline SA/V & Duration & & & & & & \\
\hline$(m-1)$ & (days) & $\mathbf{K}$ & LA & LI & MG & MN & MO \\
\hline 530 & 7 & 0.00 & 28.69 & 433.01 & 185.95 & 150.34 & 424.89 \\
\hline 530 & 14 & -19.84 & 42.51 & 510.81 & 281.88 & 231.44 & 523.31 \\
\hline 530 & 14 & -19.84 & 32.04 & 502.99 & 256.22 & 203.74 & 519.71 \\
\hline 530 & 14 & -19.84 & 39.58 & 504.79 & 273.98 & 223.52 & 518.51 \\
\hline 530 & 28 & 0.00 & 60.31 & 566.74 & 350.75 & 287.81 & 555.12 \\
\hline 530 & 28 & 0.00 & 62.41 & 567.90 & 359.26 & 296.71 & 568.96 \\
\hline 530 & 28 & 0.00 & 54.24 & 564.43 & 342.25 & 280.89 & 564.35 \\
\hline 530 & 56 & 891398.91 & 41.77 & 670.60 & 316.55 & 228.06 & 541.18 \\
\hline 530 & 56 & 0.00 & 30.25 & 658.40 & 178.73 & 132.18 & 541.18 \\
\hline 530 & 56 & 48183.72 & 61.52 & 682.79 & 361.77 & 269.02 & 551.90 \\
\hline 530 & 91 & 0.00 & 44.44 & 725.60 & 290.28 & 207.58 & 594.23 \\
\hline 530 & 91 & 0.00 & 47.73 & 737.79 & 341.96 & 244.82 & 599.59 \\
\hline 530 & 91 & 0.00 & 45.26 & 737.79 & 335.50 & 242.96 & 599.59 \\
\hline 530 & 182 & 0.00 & 50.41 & 792.67 & 342.39 & 253.20 & 616.20 \\
\hline 530 & 182 & 0.00 & 64.19 & 823.16 & 348.85 & 259.71 & 637.63 \\
\hline 530 & 182 & 0.00 & 60.49 & 835.35 & 342.39 & 259.71 & 637.63 \\
\hline 2000 & 0.25 & 368605.49 & 7.66 & 281.47 & 12.54 & 11.97 & 224.93 \\
\hline 2000 & 0.25 & 184302.75 & 8.65 & 276.17 & 14.12 & 12.46 & 222.80 \\
\hline 2000 & 0.25 & 184302.75 & 15.48 & 275.89 & 17.24 & 18.99 & 218.58 \\
\hline 2000 & 0.5 & 368605.49 & 14.30 & 348.10 & 15.67 & 21.46 & 287.19 \\
\hline 2000 & 0.5 & 0.00 & 11.81 & 341.12 & 12.54 & 20.97 & 286.68 \\
\hline 2000 & 0.5 & 0.00 & 12.65 & 290.60 & 14.12 & 18.99 & 249.98 \\
\hline 2000 & 1 & 0.00 & 18.01 & 493.43 & 44.02 & 39.56 & 450.09 \\
\hline 2000 & 1 & 0.00 & 19.90 & 509.06 & 51.91 & 41.54 & 466.30 \\
\hline 2000 & 1 & 0.00 & 23.46 & 524.69 & 60.80 & 51.43 & 481.90 \\
\hline 2000 & 3. & 0.00 & 42.72 & 774.69 & 321.56 & 209.68 & 719.55 \\
\hline 2000 & 3. & 0.00 & 34.35 & 768.67 & 299.84 & 187.92 & 719.55 \\
\hline 2000 & 3 & 0.00 & 40.21 & 768.67 & 307.74 & 199.79 & 713.55 \\
\hline 2000 & 7 & 0.00 & 65.13 & 980.01 & 524.28 & 415.40 & 863.58 \\
\hline 2000 & 7 & 0.00 & 56.34 & 967.98 & 488.75 & 375.84 & 863.58 \\
\hline 2000 & 7 & 0.00 & 51.73 & 967.98 & 506.51 & 392.65 & 863.58 \\
\hline 2000 & 14 & 0.00 & 64.50 & 1100.25 & 602.45 & 475.73 & 1001.01 \\
\hline 2000 & 14 & 0.00 & 60.52 & 1088.23 & 584.68 & 457.93 & 995.01 \\
\hline 2000 & 14 & 0.00 & 71.62 & 1082.22 & 602.45 & 482.65 & 971.00 \\
\hline 2000 & 28 & 0.00 & 62.62 & 1220.20 & 633.64 & 517.27 & 1050.22 \\
\hline 2000 & 28 & 0.00 & 81.68 & 1284.21 & 754.65 & 578.59 & 1067.53 \\
\hline 2000 & 28 & 0.00 & 91.10 & 1266.85 & 750.40 & 585.52 & 1055.99 \\
\hline 2000 & 56 & 0.00 & 106.17 & 1456.99 & 724.83 & 539.90 & 1022.89 \\
\hline 2000 & 56 & 0.00 & 101.85 & 1469.18 & 731.29 & 541.76 & 1033.61 \\
\hline 2000 & 56 & 0.00 & 110.08 & 1414.31 & 711.91 & 531.53 & 1001.46 \\
\hline 2000 & 91 & 0.00 & 146.70 & 1628.02 & 915.19 & 668.36 & 1108.09 \\
\hline 2000 & 91 & 0.00 & 151.64 & 1664.61 & 932.42 & 680.46 & 1124.16 \\
\hline 2000 & 91 & 0.00 & 140.53 & 1579.24 & 897.96 & 655.33 & 1081.30 \\
\hline 2000 & 182 & 0.00 & 222.21 & 1871.92 & 953.95 & 727.01 & 1210.97 \\
\hline 2000 & 182 & 0.00 & 240.73 & 1926.80 & 895.81 & 669.29 & 1210.97 \\
\hline 2000 & 182 & 0.00 & 234.55 & 1896.31 & 889.35 & 661.85 & 1184.18 \\
\hline 20000 & 1 & 0.00 & 46.60 & 2467.84 & 865.48 & 521.66 & 2032.57 \\
\hline
\end{tabular}


NORM. CONC. (g/m2, blank corrected) FOR 0.45u FILTERED LEACHATES (all tests)

\begin{tabular}{|r|r|r|r|r|r|r|r|}
\hline SA/V & Duration & & & & & & \\
\hline$(\mathrm{m}-1)$ & (days) & \multicolumn{1}{|c|}{ K } & \multicolumn{1}{c|}{ LA } & LI & MG & MN & \multicolumn{1}{c|}{ MO } \\
\hline 20000 & 1 & 737210.99 & 42.55 & 2486.86 & 783.56 & 449.42 & 2003.96 \\
\hline 20000 & 1 & 0.00 & 48.97 & 2789.54 & 842.13 & 465.73 & 2212.41 \\
\hline 20000 & 3 & 0.00 & 96.33 & 4055.31 & 2020.34 & 1681.38 & 3447.73 \\
\hline 20000 & 3 & 0.00 & 81.19 & 3890.09 & 1771.35 & 1487.31 & 3167.11 \\
\hline 20000 & 3 & 0.00 & 90.05 & 3814.82 & 1714.38 & 1444.01 & 3147.67 \\
\hline 20000 & 7 & -58.10 & 141.36 & 4775.27 & 2556.25 & 2250.08 & 3768.79 \\
\hline 20000 & 7 & -58.10 & 154.97 & 4745.21 & 2605.60 & 2284.70 & 3708.77 \\
\hline 20000 & 7 & -58.10 & 147.29 & 4683.65 & 2552.60 & 2252.77 & 3682.37 \\
\hline 20000 & 14 & 0.00 & 205.24 & 5651.57 & 2763.52 & 2586.36 & 4170.87 \\
\hline 20000 & 14 & 0.00 & 217.46 & 5508.48 & 2833.36 & 2648.01 & 4032.12 \\
\hline 20000 & 14 & 0.00 & 262.83 & 5501.26 & 2941.17 & 2759.44 & 3990.83 \\
\hline 20000 & 28 & 0.00 & 17.80 & 5170.98 & 14.88 & 9.89 & 4789.46 \\
\hline 20000 & 28 & 0.00 & 20.94 & 5170.98 & 12.75 & 4.95 & 4760.61 \\
\hline 20000 & 28 & 0.00 & 26.81 & 4956.90 & 21.05 & 0.00 & 4620.96 \\
\hline
\end{tabular}


NORM. CONC. (g/m2, blank corrected) FOR 0.45u FILTERED LEACHATES (all tests)

\begin{tabular}{|c|c|c|c|c|c|c|c|}
\hline SA/V & Duration & & & & & & \\
\hline$(m-1)$ & (days) & NA & ND & NI & $\mathbf{P}$ & $\overline{\mathrm{PB}}$ & $\overline{R U}$ \\
\hline 10 & 0.25 & 3.19 & 0.00 & 0.22 & -183313.12 & 32316.71 & 0.00 \\
\hline 10 & 0.25 & 3.68 & 0.00 & 0.22 & -183313.12 & 0.00 & 0.00 \\
\hline 10 & 0.25 & 3.14 & 0.00 & 0.00 & -183313.12 & 0.00 & 0.00 \\
\hline 10 & 0.5 & 11.94 & 0.00 & 0.00 & 0.00 & 0.00 & 0.00 \\
\hline 10 & 0.5 & 15.96 & 0.00 & 0.00 & 0.00 & 0.00 & 0.00 \\
\hline 10 & 0.5 & 16.77 & 0.00 & 0.00 & 0.00 & 0.00 & 0.00 \\
\hline 10 & 1 & 28.71 & 0.00 & 0.00 & 68742.42 & 0.00 & 0.00 \\
\hline 10 & 1 & 29.25 & 0.00 & 0.00 & 0.00 & 0.00 & 0.00 \\
\hline 10 & 1 & 25.61 & 0.00 & 0.00 & 0.00 & 0.00 & 0.00 \\
\hline 10 & 3 & 68.75 & 0.00 & 0.00 & 68742.42 & $\overline{0.00}$ & 0.00 \\
\hline 10 & 3 & 63.89 & 0.00 & 1.08 & -45828.28 & 0.00 & 0.00 \\
\hline 10 & 3 & 64.43 & 0.00 & 0.00 & -435368.67 & 0.00 & 0.00 \\
\hline 10 & 7 & 94.56 & 0.00 & 0.65 & -229141.40 & -215444.76 & 0.00 \\
\hline 10 & 7 & 96.99 & 0.00 & 0.65 & 320797.96 & -107722.38 & 0.00 \\
\hline 10 & 7 & 93.89 & 0.00 & 0.00 & -229141.40 & -215444.76 & 0.00 \\
\hline 10 & 14 & 116.87 & 0.00 & 1.51 & 779080.77 & -215444.76 & 26332.24 \\
\hline 10 & 14 & 117.27 & 0.00 & 0.86 & 412454.53 & -215444.76 & 0.00 \\
\hline 10 & 14 & 117.68 & 0.00 & 0.86 & 297883.82 & -323167.14 & 0.00 \\
\hline 10 & 28 & 133.11 & 0.00 & 0.86 & -229141.40 & -323167.14 & -13166.12 \\
\hline 10 & 28 & 137.29 & 0.00 & 0.86 & 45828.28 & -323167.14 & -13166.12 \\
\hline 10 & 28 & 135.94 & 0.00 & 0.65 & -229141.40 & -323167.14 & -13166.12 \\
\hline 10 & 56 & 152.32 & 0.00 & 1.08 & 206227.26 & -215444.76 & 52664.49 \\
\hline 10 & 56 & 150.97 & 0.00 & 0.86 & 595767.65 & -215444.76 & 0.00 \\
\hline 10 & 56 & 153.67 & 0.00 & 1.08 & 68742.42 & -323167.14 & 0.00 \\
\hline 10 & 91 & 171.19 & 0.00 & 0.22 & 389540.39 & -430889.52 & 0.00 \\
\hline 10 & 91 & 172.54 & 0.00 & 1.08 & 22914.14 & -430889.52 & 0.00 \\
\hline 10 & 91 & 169.85 & 0.00 & 0.86 & 0.00 & -430889.52 & 0.00 \\
\hline 10 & 182 & 193.45 & 0.00 & 0.22 & 1489419.12 & -107722.38 & 0.00 \\
\hline 10 & 182 & 188.06 & 0.78 & 0.00 & 1122792.88 & 0.00 & 26332.24 \\
\hline 10 & 182 & 198.84 & 0.00 & 0.00 & 847823.19 & -107722.38 & -131661.22 \\
\hline 10 & 364 & 205.55 & 1.56 & 3.67 & 1626903.96 & 226217.00 & -26332.24 \\
\hline 10 & 364 & 216.34 & 1.30 & 3.02 & 1901873.65 & 193900.28 & 13166.12 \\
\hline 10 & 364 & 216.34 & 1.30 & 3.02 & 1901873.65 & 193900.28 & 13166.12 \\
\hline 530 & 0.25 & 113.65 & $1 . \overline{36}$ & 9.25 & 256638.37 & 130344.08 & 5.43 \\
\hline 530 & 0.25 & 118.79 & 6.80 & 13.47 & 128319.19 & 129266.86 & 0.00 \\
\hline 530 & 0.25 & 127.36 & 12.26 & 14.74 & 0.00 & 257456.49 & 0.25 \\
\hline 530 & 0.5 & 149.25 & 19.06 & 20.19 & 64159.59 & 386723.35 & 12.67 \\
\hline 530 & 0.5 & 152.54 & 16.33 & 16.42 & 128319.19 & 256379.27 & 1.81 \\
\hline 530 & 0.5 & 153.47 & 8.16 & 12.20 & -64159.59 & -132498.53 & 0.25 \\
\hline 530 & 1 & 191.28 & 27.70 & 40.86 & 618681.79 & -430889.52 & 0.00 \\
\hline 530 & 1 & 191.28 & 20.65 & 35.27 & 572853.51 & -430889.52 & 0.00 \\
\hline 530 & 1 & 187.43 & 22.11 & 36.61 & 343712.11 & -430889.52 & 0.00 \\
\hline 530 & 3 & 288.56 & 17.25 & 43.98 & 1122792.88 & -344711.62 & 0.00 \\
\hline 530 & 3 & 292.41 & 26.49 & 53.58 & 1031136.32 & -344711.62 & 0.00 \\
\hline 530 & 3 & 288.56 & 32.56 & 60.28 & 824909.05 & -452434.00 & 0.00 \\
\hline 530 & 7 & 404.39 & 27.94 & 84.61 & 320797.96 & -538611.90 & 0.00 \\
\hline 530 & 7 & 400.54 & 28.19 & 88.86 & 114570.70 & -538611.90 & 0.00 \\
\hline
\end{tabular}


NORM. CONC. ( $\mathrm{g} / \mathrm{m} 2$, blank corrected) FOR 0.45u FILTERED LEACHATES (all tests)

\begin{tabular}{|c|c|c|c|c|c|c|c|}
\hline SA/V & Duration & & & & & & \\
\hline$(m-1)$ & (days) & NA & ND & NI & $\mathbf{P}$ & PB & $\mathrm{RU}$ \\
\hline 530 & 7 & 388.99 & 24.06 & 79.48 & 824909.05 & -538611.90 & 0.00 \\
\hline 530 & 14 & 457.77 & 38.64 & 120.34 & 1626903.96 & -689423.23 & 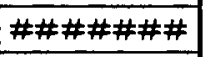 \\
\hline 530 & 14 & 451.36 & 28.19 & 99.13 & 1787302.95 & -581700.85 & \#\#\#\#\#\#\# \\
\hline 530 & 14 & 453.92 & 33.05 & 114.31 & 1764388.81 & -473978.47 & 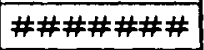 \\
\hline 530 & 28 & 535.15 & 58.82 & 145.71 & 1420676.70 & -107722.38 & 38.68 \\
\hline 530 & 28 & 537.84 & 59.81 & 142.74 & 1191535.30 & -107722.38 & 54.31 \\
\hline 530 & 28 & 531.10 & 50.13 & 129.59 & 893651.47 & -107722.38 & 45.26 \\
\hline 530 & 56 & 577.52 & 44.06 & 133.73 & 1466504.98 & -646334.28 & 26332.24 \\
\hline 530 & 56 & 568.08 & 32.14 & 81.53 & 1741474.67 & -646334.28 & 0.00 \\
\hline 530 & 56 & 586.95 & 65.58 & 166.30 & 1856045.37 & -754056.66 & 0.00 \\
\hline 530 & 91 & 636.25 & 49.51 & 130.28 & 2291414.03 & -538611.90 & 0.00 \\
\hline 530 & 91 & 645.68 & 50.80 & 153.36 & 1970616.07 & -646334.28 & 0.00 \\
\hline 530 & 91 & 644.33 & 47.17 & 149.69 & 1993530.21 & -754056.66 & 0.00 \\
\hline 530 & 182 & 661.86 & 48.99 & 159.61 & 2337242.31 & 21544.48 & 789967.35 \\
\hline 530 & 182 & 687.47 & 66.35 & 175.79 & 1741474.67 & 0.00 & 737302.86 \\
\hline 530 & 182 & 695.56 & 56.51 & 166.51 & 1466504.98 & -215444.76 & 710970.61 \\
\hline 2000 & 0.25 & 245.49 & 0.00 & 6.32 & 323089.38 & 260688.16 & 1.23 \\
\hline 2000 & 0.25 & 242.58 & 2.73 & 7.17 & 387248.97 & -1077.22 & 3.37 \\
\hline 2000 & 0.25 & 243.77 & 6.80 & 10.94 & 1097587.32 & 258533.71 & 5.02 \\
\hline 2000 & 0.5 & 306.40 & 5.43 & 11.37 & -192478.78 & 389955.02 & 0.41 \\
\hline 2000 & 0.5 & 305.34 & 5.43 & 10.54 & -128319.19 & 259610.94 & 2.80 \\
\hline 2000 & 0.5 & 264.08 & 4.09 & 9.27 & 130610.60 & 389955.02 & 0.00 \\
\hline 2000 & 1 & 401.83 & 16.52 & 31.03 & 1443590.84 & -538611.90 & 78996.73 \\
\hline 2000 & 1 & 414.66 & 18.22 & 33.71 & 1168621.16 & -538611.90 & 26332.24 \\
\hline 2000 & 1 & 424.93 & 20.65 & 39.52 & 1214449.44 & -430889.52 & 65830.61 \\
\hline 2000 & 3 & 648.31 & 32.80 & 119.89 & 801994.91 & 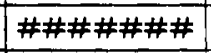 & 0.00 \\
\hline 2000 & 3 & 648.31 & 24.54 & 107.83 & 1283191.86 & $\# \# \# \# \# \#$ & 0.00 \\
\hline 2000 & 3 & 644.46 & 29.65 & 115.42 & 1214449.44 & 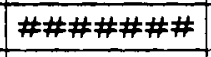 & 0.00 \\
\hline 2000 & 7 & 799.80 & 62.69 & 251.84 & 2131015.05 & -861779.04 & 0.00 \\
\hline 2000 & 7 & 794.67 & 51.76 & 225.04 & 1649818.10 & -861779.04 & 0.00 \\
\hline 2000 & 7 & 790.82 & 49.09 & 238.44 & 2085186.77 & -754056.66 & 0.00 \\
\hline 2000 & 14 & 903.52 & 62.69 & 287.78 & 2337242.31 & -861779.04 & \#\#\#\#\#\#\# \\
\hline 2000 & 14 & 890.68 & 60.02 & 278.85 & 2268499.89 & -754056.66 & 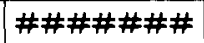 \\
\hline 2000 & 14 & 890.68 & 65.37 & 294.48 & 1924787.79 & 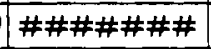 & 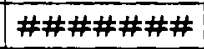 \\
\hline 2000 & 28 & 965.41 & 57.35 & 292.47 & 1833131.23 & -107722.38 & 882130.21 \\
\hline 2000 & 28 & 1072.99 & 80.16 & 313.90 & 458282.81 & -107722.38 & 70.77 \\
\hline 2000 & 28 & 1054.12 & 90.83 & 318.14 & 206227.26 & -215444.76 & 74.88 \\
\hline 2000 & 56 & 1133.60 & 121.05 & 377.03 & 3368378.63 & -980273.66 & -131661.22 \\
\hline 2000 & 56 & 1137.64 & 116.12 & 374.87 & 3391292.77 & -872551.28 & 52664.49 \\
\hline 2000 & 56 & 1101.25 & 125.97 & 379.18 & 3070494.81 & -872551.28 & 65830.61 \\
\hline 2000 & 91 & 1291.37 & 169.00 & 478.83 & 2978838.24 & -969501.42 & 0.00 \\
\hline 2000 & 91 & 1319.67 & 178.07 & 502.56 & 3345464.49 & -969501.42 & 0.00 \\
\hline 2000 & 91 & 1256.32 & 164.33 & 485.30 & 3643348.31 & -754056.66 & 0.00 \\
\hline 2000 & 182 & 1388.42 & 259.20 & 545.70 & 3414206.91 & 96950.14 & 2738553.48 \\
\hline 2000 & 182 & 1388.42 & 279.93 & 517.66 & 3803747.30 & 43088.95 & 2211908.58 \\
\hline 2000 & 182 & 1361.46 & 274.75 & 513.34 & 3666262.45 & 64633.43 & 2198742.46 \\
\hline 20000 & 1 & 1919.96 & 21.75 & 320.25 & 4128492.45 & -2201.20 & 50.71 \\
\hline
\end{tabular}


NORM. CONC. (g/m2, blank corrected) FOR 0.45u FILTERED LEACHATES (all tests)

\begin{tabular}{|r|r|r|r|r|r|r|r|}
\hline SA/V & Duration & & & & & & \\
\hline (m-1) & (days) & NA & ND & NI & P & PB & RU \\
\hline 20000 & 1 & 1916.45 & 27.00 & 277.93 & 4124545.26 & 1555511.17 & 36.87 \\
\hline 20000 & 1 & 2141.10 & 12.54 & 292.81 & 4248121.22 & 691230.82 & 24.19 \\
\hline 20000 & 3 & 2976.92 & 76.54 & 1019.18 & 5499393.68 & $\# \# \# \# \# \#$ & 0.00 \\
\hline 20000 & 3 & 2831.45 & 35.20 & 934.59 & 5075940.37 & $\# \# \# \# \#$ & 0.00 \\
\hline 20000 & 3 & 2790.77 & 53.46 & 904.21 & 4353686.66 & $\# \# \# \# \#$ & 0.00 \\
\hline 20000 & 7 & 3404.61 & 147.01 & 1438.90 & 7561666.31 & $\# \# \# \# \# \#$ & 0.00 \\
\hline 20000 & 7 & 3372.52 & 156.73 & 1472.39 & 5270252.28 & $\# \# \# \# \#$ & 0.00 \\
\hline 20000 & 7 & 3341.76 & 152.40 & 1453.43 & 5966842.14 & $\# \# \# \# \#$ & 0.00 \\
\hline 20000 & 14 & 3883.47 & 200.47 & 1607.46 & 5957676.49 & $\# \# \# \# \# \#$ & 8821302.07 \\
\hline 20000 & 14 & 3763.87 & 209.03 & 1647.76 & 7347877.38 & $\# \# \# \# \# \#$ & 7840820.93 \\
\hline 20000 & 14 & 3742.25 & 262.44 & 1752.58 & 6301388.59 & $\# \# \# \# \#$ & 8689640.84 \\
\hline 20000 & 28 & 4241.02 & 0.00 & 2.12 & 6988812.80 & 0.00 & 0.00 \\
\hline 20000 & 28 & 4267.98 & 0.00 & 0.00 & 6301388.59 & 0.00 & 0.00 \\
\hline 20000 & 28 & 4131.83 & 0.00 & 0.00 & 4032888.70 & 0.00 & 27.16 \\
\hline
\end{tabular}


NORM. CONC. (g/m2, blank corrected) FOR 0.45u FILTERED LEACHATES (all tests)

\begin{tabular}{|c|c|c|c|c|c|c|c|}
\hline $\mathrm{SA} / \mathrm{V}$ & Duration & & & & & & \\
\hline$(m-1)$ & (days) & $\widehat{\text { SB }}$ & SI & SR & $\mathrm{TE}$ & $\overline{T I}$ & $\mathrm{~V}$ \\
\hline 10 & 0.25 & 0.00 & 2.59 & 1.48 & 0.00 & 0.00 & 0.00 \\
\hline 10 & 0.25 & 0.00 & 3.30 & 1.48 & 0.00 & 0.00 & 0.00 \\
\hline 10 & 0.25 & 0.00 & 3.48 & 1.48 & 125078.37 & 0.00 & 0.00 \\
\hline 10 & 0.5 & 0.00 & 12.38 & 10.35 & 0.00 & 0.00 & 0.00 \\
\hline 10 & 0.5 & 0.00 & 16.12 & 13.30 & 0.00 & 0.00 & 0.00 \\
\hline 10 & 0.5 & 0.00 & 17.06 & 13.30 & 0.00 & 0.00 & 0.00 \\
\hline 10 & 1 & 0.00 & 27.38 & 20.70 & 0.00 & 0.00 & 0.00 \\
\hline 10 & 1 & 0.00 & 28.55 & 22.17 & 0.00 & 0.00 & 0.00 \\
\hline 10 & 1 & 0.00 & 26.09 & 20.70 & 0.00 & 0.00 & 0.00 \\
\hline 10 & 3 & 59856.26 & 63.28 & 48.78 & 0.00 & 0.00 & 0.00 \\
\hline 10 & 3 & 71827.52 & 59.11 & 47.30 & 12507.84 & 0.00 & 0.00 \\
\hline 10 & 3 & 47885.01 & 58.28 & 47.30 & 0.00 & 0.00 & 0.00 \\
\hline 10 & 7 & 119712.53 & 81.21 & 56.17 & -162601.88 & 0.00 & 0.00 \\
\hline 10 & 7 & 107741.27 & 82.46 & 56.17 & -37523.51 & 0.00 & 0.00 \\
\hline 10 & 7 & 119712.53 & 79.54 & 54.70 & -162601.88 & 0.00 & 0.00 \\
\hline 10 & 14 & 0.00 & 96.72 & 56.17 & -125078.37 & 0.00 & 0.00 \\
\hline 10 & 14 & 0.00 & 97.13 & 56.17 & -125078.37 & 0.00 & 0.00 \\
\hline 10 & 14 & 0.00 & 97.55 & 56.17 & -250156.74 & 0.00 & 0.00 \\
\hline 10 & 28 & 71827.52 & 110.90 & 57.65 & -125078.37 & 0.00 & 0.00 \\
\hline 10 & 28 & 23942.51 & 112.99 & 56.17 & -125078.37 & 0.00 & 0.00 \\
\hline 10 & 28 & 35913.76 & 112.57 & 57.65 & -125078.37 & 0.00 & 0.00 \\
\hline 10 & 56 & 119712.53 & 124.20 & 59.13 & 87554.86 & 0.00 & 14711.24 \\
\hline 10 & 56 & 95770.02 & 122.11 & 57.65 & 62539.18 & 0.00 & 0.00 \\
\hline 10 & 56 & 47885.01 & 123.78 & 57.65 & 0.00 & 0.00 & 0.00 \\
\hline 10 & 91 & 0.00 & 133.29 & 59.13 & -375235.11 & 0.00 & 0.00 \\
\hline 10 & 91 & 11971.25 & 134.12 & 60.61 & -375235.11 & 0.00 & 0.00 \\
\hline 10 & 91 & 0.00 & 133.29 & 59.13 & -250156.74 & 0.00 & 0.00 \\
\hline 10 & 182 & 11971.25 & 146.78 & 57.65 & -250156.74 & 8.34 & 0.00 \\
\hline 10 & 182 & 0.00 & 143.86 & 57.65 & -125078.37 & 0.00 & 0.00 \\
\hline 10 & 182 & 0.00 & 152.62 & 57.65 & -250156.74 & 0.00 & 0.00 \\
\hline 10 & 364 & 323223.82 & 162.92 & 59.13 & -75047.02 & 0.00 & 14711.24 \\
\hline 10 & 364 & 0.00 & 170.84 & 57.65 & -200125.39 & 0.00 & 14711.24 \\
\hline 10 & 364 & 0.00 & 170.84 & 57.65 & -200125.39 & 0.00 & 14711.24 \\
\hline 530 & 0.25 & 208299.79 & 95.19 & 37.40 & 1195749.22 & 0.00 & 0.00 \\
\hline 530 & 0.25 & 208299.79 & 100.33 & 40.21 & 1992498.43 & 0.00 & 0.00 \\
\hline 530 & 0.25 & 104149.90 & 106.24 & 41.10 & 796749.22 & 0.00 & 0.00 \\
\hline 530 & 0.5 & -104149.90 & 121.03 & 37.70 & -397749.22 & 1.67 & 0.00 \\
\hline 530 & 0.5 & 104149.90 & 122.70 & 35.03 & 399000.00 & 0.00 & 0.00 \\
\hline 530 & 0.5 & 104149.90 & 121.16 & 31.93 & $\# \# \# \# \# \# \#$ & 0.00 & 0.00 \\
\hline 530 & 1 & 0.00 & 145.74 & 43.93 & -250156.74 & 22.24 & 0.00 \\
\hline 530 & 1 & -119712.53 & 143.60 & 38.86 & -250156.74 & 16.68 & 0.00 \\
\hline 530 & 1 & 0.00 & 141.45 & 40.55 & -250156.74 & 22.24 & 0.00 \\
\hline 530 & 3 & 131683.78 & 206.60 & 35.48 & -125078.37 & 22.24 & 44133.72 \\
\hline 530 & 3 & 263367.56 & 212.17 & 42.24 & -125078.37 & 27.80 & 44133.72 \\
\hline 530 & 3 & 179568.79 & 211.31 & 45.61 & -375235.11 & 33.36 & 58844.96 \\
\hline 530 & 7 & 203511.29 & 269.24 & 65.89 & 175109.72 & 44.48 & 44133.72 \\
\hline 530 & 7 & 179568.79 & 270.53 & 64.20 & -250156.74 & 44.48 & 44133.72 \\
\hline
\end{tabular}


NORM. CONC. (g/m2, blank corrected) FOR 0.45u FILTERED LEACHATES (all tests)

\begin{tabular}{|c|c|c|c|c|c|c|c|}
\hline $\mathrm{SA} / \mathrm{V}$ & Duration & & & & & & \\
\hline$(m-1)$ & (days) & SB & SI & SR & TE & TI & V \\
\hline 530 & 7 & 287310.06 & 258.09 & 59.13 & -125078.37 & 38.92 & 29422.48 \\
\hline 530 & 14 & 155626.28 & 314.26 & 86.16 & -125078.37 & 50.04 & 58844.96 \\
\hline 530 & 14 & 155626.28 & 308.68 & 77.71 & -250156.74 & 44.48 & 58844.96 \\
\hline 530 & 14 & 143655.03 & 310.83 & 84.47 & -375235.11 & 44.48 & 58844.96 \\
\hline 530 & 28 & 586591.38 & 342.12 & 100.52 & -250156.74 & 54.21 & 1.18 \\
\hline 530 & 28 & 598562.63 & 429.31 & 100.52 & -375235.11 & 62.55 & 1.18 \\
\hline 530 & 28 & 574620.12 & 421.23 & 96.09 & -375235.11 & 54.21 & 1.18 \\
\hline 530 & 56 & 490821.36 & 335.15 & 90.17 & 425266.46 & 91.74 & 88267.44 \\
\hline 530 & 56 & 131683.78 & 316.80 & 57.65 & 312695.92 & 50.04 & 58844.96 \\
\hline 530 & 56 & 442936.34 & 343.07 & 106.43 & 500313.48 & 116.76 & 88267.44 \\
\hline 530 & 91 & 263367.56 & 356.09 & 87.22 & 212633.23 & 91.74 & 102978.68 \\
\hline 530 & 91 & 239425.05 & 366.52 & 99.04 & 37523.51 & 108.42 & 88267.44 \\
\hline 530 & 91 & 239425.05 & 365.68 & 97.57 & 187617.55 & 91.74 & 88267.44 \\
\hline 530 & 182 & 430965.09 & 393.68 & 104.96 & 87554.86 & 91.74 & 29422.48 \\
\hline 530 & 182 & 299281.31 & 397.43 & 118.26 & -125078.37 & 100.08 & 29422.48 \\
\hline 530 & 182 & 191540.04 & 397.01 & 112.35 & -125078.37 & 83.40 & 0.00 \\
\hline 2000 & 0.25 & -520749.49 & 159.46 & 8.72 & -397749.22 & 0.00 & 0.00 \\
\hline 2000 & 0.25 & -208299.79 & 159.07 & 9.17 & -796749.22 & 0.00 & 0.00 \\
\hline 2000 & 0.25 & -312449.69 & 162.42 & 13.30 & -796749.22 & 0.00 & 0.00 \\
\hline 2000 & 0.5 & 104149.90 & 195.58 & 10.35 & 3584746.08 & 0.00 & 0.00 \\
\hline 2000 & 0.5 & 104149.90 & 195.19 & 9.46 & 1194498.43 & 0.00 & 0.00 \\
\hline 2000 & 0.5 & -104149.90 & 175.78 & 10.64 & 1991247.65 & 0.00 & 0.00 \\
\hline 2000 & 1 & 107741.27 & 246.93 & 16.89 & 537836.99 & 11.12 & 14711.24 \\
\hline 2000 & 1 & 119712.53 & 252.50 & 16.89 & 337711.60 & 16.68 & 29422.48 \\
\hline 2000 & 1 & 119712.53 & 261.07 & 18.58 & 650407.52 & 16.68 & 29422.48 \\
\hline 2000 & 3 & 47885.01 & 357.54 & 42.24 & 0.00 & 38.92 & 0.00 \\
\hline 2000 & 3 & 215482.55 & 357.97 & 35.48 & 125078.37 & 27.80 & 0.00 \\
\hline 2000 & 3 & 59856.26 & 355.82 & 40.55 & 0.00 & 33.36 & 0.00 \\
\hline 2000 & 7 & 311252.57 & 423.14 & 69.27 & 175109.72 & 111.20 & 117689.92 \\
\hline 2000 & 7 & 359137.58 & 418.00 & 60.82 & 337711.60 & 100.08 & 102978.68 \\
\hline 2000 & 7 & 143655.03 & 420.57 & 62.51 & 387742.95 & 100.08 & 102978.68 \\
\hline 2000 & 14 & 167597.54 & 467.30 & 86.16 & 100062.70 & 105.64 & 132401.16 \\
\hline 2000 & 14 & 730246.41 & 458.73 & 82.78 & 262664.58 & 100.08 & 132401.16 \\
\hline 2000 & 14 & 155626.28 & 454.44 & 91.23 & -287680.25 & 100.08 & 73556.20 \\
\hline 2000 & 28 & 718275.15 & 488.47 & 96.30 & 800501.57 & 105.64 & 29422.48 \\
\hline 2000 & 28 & 670390.14 & 497.35 & 94.61 & 437774.29 & 83.40 & 1.77 \\
\hline 2000 & 28 & 694332.65 & 497.35 & 102.00 & 312695.92 & 87.57 & 2.35 \\
\hline 2000 & 56 & 670390.14 & 471.18 & 112.35 & 1175736.68 & 233.53 & 161823.64 \\
\hline 2000 & 56 & 658418.89 & 475.35 & 107.91 & 1175736.68 & 233.53 & 176534.88 \\
\hline 2000 & 56 & 766160.16 & 471.18 & 118.26 & 1125705.33 & 241.87 & 176534.88 \\
\hline 2000 & 91 & 682361.40 & 508.77 & 158.17 & -87554.86 & 225.19 & 205957.36 \\
\hline 2000 & 91 & 790102.67 & 512.94 & 167.04 & 50031.35 & 225.19 & 220668.60 \\
\hline 2000 & 91 & 873901.44 & 508.77 & 159.65 & 362727.27 & 275.23 & 235379.84 \\
\hline 2000 & 182 & 562648.87 & 621.37 & 212.87 & 250156.74 & 250.21 & 132401.16 \\
\hline 2000 & 182 & 562648.87 & 554.65 & 224.70 & 412758.62 & 158.47 & 117689.92 \\
\hline 2000 & 182 & 586591.38 & 554.65 & 226.17 & 662915.36 & 150.13 & 102978.68 \\
\hline 20000 & 1 & 1251111.06 & 802.21 & 30.17 & 7978598.62 & 38.38 & 0.00 \\
\hline
\end{tabular}


NORM. CONC. (g/m2, blank corrected) FOR 0.45u FILTERED LEACHATES (all tests)

\begin{tabular}{|r|r|r|r|r|r|r|r|}
\hline SA/V & Duration & & & & & \\
\hline$(\mathrm{m}-1)$ & (days) & \multicolumn{1}{|c|}{ SB } & \multicolumn{1}{c|}{ SI } & SR & \multicolumn{1}{c|}{ TE } & \multicolumn{1}{c|}{ TI } & \multicolumn{1}{c|}{ V } \\
\hline 20000 & 1 & 2499597.54 & 795.16 & 25.43 & 9560990.60 & 15.01 & 0.00 \\
\hline 20000 & 1 & 725924.78 & 906.17 & 29.20 & 8976601.94 & 0.00 & 0.00 \\
\hline 20000 & 3 & 659137.17 & 1189.38 & 67.58 & 3812388.71 & 361.41 & 147112.40 \\
\hline 20000 & 3 & -178850.51 & 1113.75 & 64.77 & 2807509.09 & 284.24 & 0.00 \\
\hline 20000 & 3 & -118994.25 & 1150.80 & 67.58 & 2936840.13 & 305.81 & 0.00 \\
\hline 20000 & 7 & 658418.89 & 1424.39 & 101.37 & 8755485.89 & 639.42 & 367781.01 \\
\hline 20000 & 7 & 1376694.05 & 1405.09 & 101.37 & 8067554.86 & 667.22 & 441337.21 \\
\hline 20000 & 7 & 1176175.56 & 1329.49 & 95.33 & 7849293.10 & 656.77 & 381168.23 \\
\hline 20000 & 14 & 718275.15 & 1652.60 & 118.26 & 8130094.04 & 973.03 & 147112.40 \\
\hline 20000 & 14 & 548403.08 & 1603.79 & 120.39 & 8021776.18 & 962.25 & 74880.21 \\
\hline 20000 & 14 & 957700.21 & 1571.14 & 143.60 & 7192006.27 & 1028.64 & 147112.40 \\
\hline 20000 & 28 & 957700.21 & 1231.02 & 7.39 & 3502194.36 & 0.00 & 5.88 \\
\hline 20000 & 28 & 957700.21 & 1231.02 & 7.39 & 2063793.10 & 0.00 & 2.94 \\
\hline 20000 & 28 & 1316837.78 & 1216.14 & 8.87 & 2338965.52 & 45.87 & 6.47 \\
\hline
\end{tabular}


NORM. CONC. (g/m2, blank corrected) FOR 0.45u FILTERED LEACHATES (all tests)

\begin{tabular}{|c|c|c|c|c|}
\hline $\mathrm{SA} / \mathrm{V}$ & Duration & & & \\
\hline$(m-1)$ & (days) & $\bar{Y}$ & $\overline{\mathrm{ZN}}$ & $\overline{Z R}$ \\
\hline 10 & 0.25 & 0.00 & -236504.51 & 0.00 \\
\hline 10 & 0.25 & 0.00 & -236504.51 & 0.00 \\
\hline 10 & 0.25 & 0.00 & -236504.51 & 0.00 \\
\hline 10 & 0.5 & 0.00 & 0.00 & 0.00 \\
\hline 10 & 0.5 & 0.00 & 12447.61 & 0.00 \\
\hline 10 & 0.5 & 0.00 & 0.00 & 0.00 \\
\hline 10 & 1 & 0.00 & 0.00 & 0.00 \\
\hline 10 & 1 & 0.00 & 0.00 & 0.00 \\
\hline 10 & 1 & 0.00 & 0.00 & 0.00 \\
\hline 10 & 3 & 0.00 & 0.00 & 0.00 \\
\hline 10 & 3 & 0.00 & 0.00 & 0.00 \\
\hline 10 & 3 & 0.00 & 0.00 & 0.00 \\
\hline 10 & 7 & 0.00 & -37342.82 & 0.00 \\
\hline 10 & 7 & 0.00 & -24895.21 & 0.24 \\
\hline 10 & 7 & 0.00 & -37342.82 & 0.00 \\
\hline 10 & 14 & 0.00 & -12447.61 & 0.00 \\
\hline 10 & 14 & 0.00 & -12447.61 & 0.00 \\
\hline 10 & 14 & 0.00 & -12447.61 & 0.00 \\
\hline 10 & 28 & 0.00 & -24895.21 & -2.89 \\
\hline 10 & 28 & 0.00 & -12447.61 & -2.41 \\
\hline 10 & 28 & 0.00 & 12447.61 & -2.41 \\
\hline 10 & 56 & 0.00 & -435666.21 & 0.24 \\
\hline 10 & 56 & 0.00 & -124476.06 & 0.00 \\
\hline 10 & 56 & 0.00 & -460561.42 & 0.00 \\
\hline 10 & 91 & 0.00 & 0.00 & 1.45 \\
\hline 10 & 91 & 0.00 & 0.00 & 2.65 \\
\hline 10 & 91 & 0.00 & 0.00 & 1.21 \\
\hline 10 & 182 & 0.00 & 0.00 & 0.00 \\
\hline 10 & 182 & 0.00 & 0.00 & 0.00 \\
\hline 10 & 182 & 0.00 & 0.00 & 0.00 \\
\hline 10 & 364 & 0.00 & 12447.61 & 0.00 \\
\hline 10 & 364 & 0.00 & 0.00 & 0.00 \\
\hline 10 & 364 & 0.00 & 0.00 & 0.00 \\
\hline 530 & 0.25 & 0.00 & 0.00 & 6.68 \\
\hline 530 & 0.25 & 0.00 & 0.00 & 11.39 \\
\hline 530 & 0.25 & 0.00 & 0.00 & 12.30 \\
\hline 530 & 0.5 & 0.00 & 0.00 & 20.96 \\
\hline 530 & 0.5 & 0.00 & 7468.56 & 17.77 \\
\hline 530 & 0.5 & 0.00 & 0.00 & 11.55 \\
\hline 530 & 1 & 34.92 & 12447.61 & 37.21 \\
\hline 530 & 1 & 28.57 & 24895.21 & 31.09 \\
\hline 530 & 1 & 28.57 & 0.00 & 33.13 \\
\hline 530 & 3 & 22.22 & 37342.82 & 22.43 \\
\hline 530 & 3 & 31.75 & 37342.82 & 30.07 \\
\hline 530 & 3 & 38.10 & 49790.42 & 35.17 \\
\hline 530 & 7 & 34.92 & 24895.21 & 30.58 \\
\hline 530 & 7 & 34.92 & 12447.61 & 33.90 \\
\hline
\end{tabular}


NORM. CONC. (g/m2, blank corrected) FOR 0.45u FILTERED LEACHATES (all tests)

\begin{tabular}{|c|c|c|c|c|}
\hline $\mathrm{SA} / \mathrm{V}$ & Duration & & & \\
\hline$(m-1)$ & (days) & $\mathbf{Y}$ & $\mathbf{Z N}$ & ZR \\
\hline 530 & 7 & 31.75 & 12447.61 & 28.80 \\
\hline 530 & 14 & 50.80 & 24895.21 & 41.29 \\
\hline 530 & 14 & 38.10 & 37342.82 & 36.45 \\
\hline 530 & 14 & 44.45 & 37342.82 & 41.54 \\
\hline 530 & 28 & 11.18 & 49790.42 & 58.92 \\
\hline 530 & 28 & 11.18 & 24895.21 & 95.95 \\
\hline 530 & 28 & 9.65 & 49790.42 & 78.72 \\
\hline 530 & 56 & 53.97 & 49790.42 & 55.72 \\
\hline 530 & 56 & 38.10 & 24895.21 & 40.52 \\
\hline 530 & 56 & 76.20 & 37342.82 & 83.22 \\
\hline 530 & 91 & 60.32 & 0.00 & 54.76 \\
\hline 530 & 91 & 63.50 & 49790.42 & 58.62 \\
\hline 530 & 91 & 60.32 & 24895.21 & 56.69 \\
\hline 530 & 182 & 66.67 & 99580.85 & 60.54 \\
\hline 530 & 182 & 85.72 & 74685.64 & 60.06 \\
\hline 530 & 182 & 79.37 & 62238.03 & 62.47 \\
\hline 2000 & 0.25 & 0.00 & 0.00 & 5.31 \\
\hline 2000 & 0.25 & 0.00 & 0.00 & 5.47 \\
\hline 2000 & 0.25 & 0.00 & 0.00 & 9.87 \\
\hline 2000 & 0.5 & 0.00 & 0.00 & 9.71 \\
\hline 2000 & 0.5 & 0.00 & 0.00 & 8.66 \\
\hline 2000 & 0.5 & 0.00 & 0.00 & 8.66 \\
\hline 2000 & 1 & 19.05 & 24895.21 & 19.12 \\
\hline 2000 & 1 & 19.05 & 24895.21 & 18.35 \\
\hline 2000 & 1 & 22.22 & 24895.21 & 21.66 \\
\hline 2000 & 3 & 41.27 & 24895.21 & 40.27 \\
\hline 2000 & 3 & 34.92 & 124476.06 & 35.94 \\
\hline 2000 & 3 & 38.10 & 37342.82 & 38.99 \\
\hline 2000 & 7 & 76.20 & 99580.85 & 51.48 \\
\hline 2000 & 7 & 66.67 & 74685.64 & 60.66 \\
\hline 2000 & 7 & 63.50 & 87133.24 & 48.42 \\
\hline 2000 & 14 & 79.37 & 49790.42 & 63.21 \\
\hline 2000 & 14 & 76.20 & 37342.82 & 65.25 \\
\hline 2000 & 14 & 85.72 & 37342.82 & 67.54 \\
\hline 2000 & 28 & 76.20 & 74685.64 & 89.97 \\
\hline 2000 & 28 & 20.32 & 62238.03 & 75.69 \\
\hline 2000 & 28 & 22.35 & 74685.64 & 63.58 \\
\hline 2000 & 56 & 146.04 & 87133.24 & 150.04 \\
\hline 2000 & 56 & 139.69 & 87133.24 & 136.53 \\
\hline 2000 & 56 & 152.39 & 74685.64 & 142.56 \\
\hline 2000 & 91 & 209.54 & 186714.09 & 76.71 \\
\hline 2000 & 91 & 222.24 & 124476.06 & 49.69 \\
\hline 2000 & 91 & 206.37 & 124476.06 & 67.78 \\
\hline 2000 & 182 & 323.84 & 149371.27 & 105.89 \\
\hline 2000 & 182 & 352.41 & 161818.88 & 13.27 \\
\hline 2000 & 182 & 346.06 & 112028.45 & 12.30 \\
\hline 20000 & 1 & 0.00 & 0.00 & 36.44 \\
\hline
\end{tabular}


NORM. CONC. (g/m2, blank corrected) FOR 0.45u FILTERED LEACHATES (all tests)

\begin{tabular}{|r|r|r|r|r|}
\hline SA/V & Duration & & & \\
\hline$(\mathrm{m}-1)$ & (days) & \multicolumn{1}{|c|}{$\mathrm{Y}$} & \multicolumn{1}{|c|}{ ZN } & \multicolumn{1}{c|}{ ZR } \\
\hline 20000 & 1 & 0.00 & 54769.47 & 32.79 \\
\hline 20000 & 1 & 0.00 & 0.00 & 39.05 \\
\hline 20000 & 3 & 142.87 & 311688.05 & 168.21 \\
\hline 20000 & 3 & 108.20 & 353263.06 & 153.09 \\
\hline 20000 & 3 & 127.00 & 187211.99 & 160.57 \\
\hline 20000 & 7 & 238.12 & 684618.33 & 277.81 \\
\hline 20000 & 7 & 253.99 & 684618.33 & 279.08 \\
\hline 20000 & 7 & 244.72 & 576324.15 & 248.45 \\
\hline 20000 & 14 & 365.11 & 248952.12 & 412.89 \\
\hline 20000 & 14 & 387.84 & 387991.88 & 403.45 \\
\hline 20000 & 14 & 460.36 & 373428.18 & 397.59 \\
\hline 20000 & 28 & 0.00 & 0.00 & 27.95 \\
\hline 20000 & 28 & 2.54 & 62238.03 & 25.62 \\
\hline 20000 & 28 & 0.00 & 2900292.18 & 50.07 \\
\hline
\end{tabular}



ERAGE CONC. (ppm, blank corrected) FOR 18A FILTERED LEACHATES

\begin{tabular}{|c|c|c|c|c|c|c|c|}
\hline $\mathrm{SA} / \mathrm{V}$ & Duration & & & & & & \\
\hline$(m-1)$ & (days) & pH final & $\mathrm{pH}$ in & $\mathrm{AL}$ & AS & B & BA \\
\hline 10 & 0.25 & 7.36 & 5.58 & 0.08 & 0.00 & 0.10 & 0.00 \\
\hline 10 & 0.5 & 9.02 & 5.88 & 0.34 & 0.00 & 0.48 & 0.00 \\
\hline 10 & 1 & 9.51 & 5.67 & 0.64 & -0.01 & 0.91 & 0.01 \\
\hline 10 & 3 & 9.67 & 5.37 & 1.37 & 0.00 & 2.05 & 0.01 \\
\hline 10 & 7 & 9.52 & 5.74 & 1.66 & 0.01 & 2.89 & 0.01 \\
\hline 10 & 14 & 9.71 & 5.74 & 1.86 & -0.01 & 3.53 & 0.01 \\
\hline 10 & 28 & 9.32 & 6.01 & 2.04 & 0.01 & 4.33 & 0.01 \\
\hline 10 & 56 & 9.44 & 6.01 & 2.11 & 0.02 & 4.85 & 0.01 \\
\hline 10 & 91 & 9.33 & 6.01 & 2.09 & 0.00 & 5.29 & 0.01 \\
\hline 10 & 182 & 9.44 & 6.01 & 2.13 & -0.01 & 5.89 & 0.01 \\
\hline 10 & 364 & 8.72 & 6.00 & 1.89 & 0.01 & 6.83 & 0.01 \\
\hline 530 & 0.25 & 9.90 & 5.58 & 1.63 & 0.01 & 3.31 & 0.00 \\
\hline 530 & 0.5 & 9.96 & 5.88 & 1.77 & 0.01 & 4.24 & 0.00 \\
\hline 530 & 1 & 9.95 & 5.74 & 1.84 & -0.01 & 5.78 & 0.00 \\
\hline 530 & 3 & 9.99 & 5.51 & 2.14 & 0.01 & 9.03 & 0.00 \\
\hline 530 & 7 & 10.09 & 5.64 & 2.05 & 0.00 & 12.16 & -0.01 \\
\hline 530 & 14 & 9.99 & 5.69 & 1.86 & -0.01 & 14.53 & 0.00 \\
\hline 530 & 28 & 10.21 & 5.55 & 1.73 & 0.00 & 15.68 & 0.00 \\
\hline 530 & 56 & 10.10 & 5.61 & 1.34 & 0.01 & 17.22 & 0.00 \\
\hline 530 & 91 & 9.88 & 5.61 & 1.54 & 0.00 & 18.77 & -0.01 \\
\hline 530 & 182 & 9.96 & 5.61 & 1.14 & 0.00 & 20.00 & 0.00 \\
\hline 2000 & 0.25 & 10.15 & 5.58 & 2.05 & 0.01 & 6.22 & 0.00 \\
\hline 2000 & 0.5 & 10.18 & 5.88 & 1.99 & 0.00 & 7.87 & 0.00 \\
\hline 2000 & 1 & 10.04 & 5.69 & 2.10 & 0.00 & 12.69 & 0.00 \\
\hline 2000 & 3 & 10.31 & 5.51 & 1.94 & -0.02 & 20.46 & 0.00 \\
\hline 2000 & 7 & 10.38 & 5.74 & 1.98 & 0.00 & 25.83 & 0.00 \\
\hline 2000 & 14 & 10.28 & 5.69 & 1.76 & 0.01 & 28.63 & 0.00 \\
\hline 2000 & 28 & 10.55 & 5.55 & 1.63 & 0.00 & 31.02 & -0.01 \\
\hline 2000 & 56 & 10.42 & 5.61 & 1.39 & 0.01 & 33.99 & 0.00 \\
\hline 2000 & 91 & 10.27 & 5.61 & 1.66 & 0.00 & 36.83 & 0.00 \\
\hline 2000 & 182 & 10.35 & 5.61 & 1.40 & 0.01 & 41.83 & 0.00 \\
\hline 20000 & 1 & 10.78 & 5.58 & 1.07 & 0.06 & 63.56 & 0.00 \\
\hline 20000 & 3 & 10.89 & 5.51 & 0.39 & -0.02 & 99.47 & 0.00 \\
\hline 20000 & 7 & 10.89 & 5.64 & 0.55 & -0.05 & 115.40 & -0.01 \\
\hline 20000 & 14 & 10.93 & 5.64 & 0.57 & 0.00 & 131.08 & 0.00 \\
\hline 20000 & 28 & 11.20 & 5.55 & 0.49 & 0.01 & 149.30 & 0.00 \\
\hline
\end{tabular}


AVERAGE CONC. (ppm, blank corrected) FOR 18A FILTERED LEACHATES

\begin{tabular}{|c|c|c|c|c|c|c|c|}
\hline SA/V & Duration & & & & & & \\
\hline$(m-1)$ & (days) & $\overline{\mathrm{BE}}$ & $\overline{\mathrm{CA}}$ & $C D$ & $\overline{C E}$ & $\mathrm{CO}$ & CR \\
\hline 10 & 0.25 & 0.00 & 0.03 & 0.00 & 0.00 & 0.00 & 0.00 \\
\hline 10 & 0.5 & 0.00 & 0.26 & 0.00 & 0.00 & 0.00 & 0.00 \\
\hline 10 & 1 & 0.00 & 0.47 & 0.00 & 0.00 & 0.00 & 0.00 \\
\hline 10 & 3 & 0.00 & 1.08 & 0.00 & 0.00 & 0.00 & 0.00 \\
\hline 10 & 7 & 0.00 & 1.29 & 0.00 & 0.00 & 0.00 & 0.00 \\
\hline 10 & 14 & 0.00 & 1.35 & 0.00 & 0.00 & 0.00 & 0.00 \\
\hline 10 & 28 & 0.00 & 1.49 & 0.00 & 0.00 & 0.00 & 0.00 \\
\hline 10 & 56 & 0.00 & 1.52 & 0.00 & 0.00 & 0.00 & 0.01 \\
\hline 10 & 91 & 0.00 & 1.47 & 0.00 & 0.00 & 0.00 & 0.01 \\
\hline 10 & 182 & 0.00 & 1.79 & 0.00 & 0.00 & 0.00 & 0.01 \\
\hline 10 & 364 & 0.00 & 1.83 & 0.00 & 0.01 & 0.00 & 0.01 \\
\hline 530 & 0.25 & 0.00 & 0.42 & 0.00 & 0.00 & 0.00 & 0.00 \\
\hline 530 & 0.5 & 0.00 & 0.39 & 0.00 & 0.00 & 0.00 & 0.00 \\
\hline 530 & 1 & 0.00 & 0.22 & 0.00 & 0.00 & 0.00 & 0.00 \\
\hline 530 & 3 & 0.00 & 0.13 & 0.00 & 0.00 & 0.00 & 0.01 \\
\hline 530 & 7 & 0.00 & 0.15 & 0.00 & 0.00 & 0.00 & 0.02 \\
\hline 530 & 14 & 0.00 & 0.19 & 0.10 & 0.00 & 0.00 & 0.02 \\
\hline 530 & 28 & 0.00 & 0.29 & 0.00 & 0.00 & 0.00 & 0.03 \\
\hline 530 & 56 & 0.00 & 0.20 & 0.00 & 0.00 & 0.00 & 0.04 \\
\hline 530 & 91 & 0.00 & 0.37 & 0.00 & 0.00 & 0.00 & 0.05 \\
\hline 530 & 182 & 0.00 & 0.50 & 0.00 & 0.00 & 0.00 & 0.06 \\
\hline 2000 & 0.25 & 0.00 & 0.05 & 0.00 & 0.00 & 0.00 & 0.00 \\
\hline 2000 & 0.5 & 0.00 & 0.06 & 0.00 & 0.00 & 0.00 & 0.00 \\
\hline 2000 & 1 & 0.00 & 0.02 & 0.00 & 0.00 & 0.00 & 0.01 \\
\hline 2000 & 3 & 0.00 & 0.06 & 0.00 & 0.00 & 0.00 & 0.02 \\
\hline 2000 & 7 & 0.00 & 0.16 & 0.00 & 0.00 & 0.00 & 0.04 \\
\hline 2000 & 14 & 0.00 & 0.16 & 0.40 & 0.00 & 0.00 & 0.04 \\
\hline 2000 & 28 & 0.00 & 0.22 & 0.00 & 0.00 & 0.00 & 0.06 \\
\hline 2000 & 56 & 8.88 & 8.31 & B.B & B.8B & A. 8 B & $979 \times 8.99$ \\
\hline 2000 & 91 & 0.00 & 0.72 & 0.00 & 0.00 & 0.00 & 0.12 \\
\hline 2000 & 182 & & & & & & \\
\hline 20000 & 1 & 0.00 & 0.09 & 0.00 & 0.00 & 0.00 & 0.03 \\
\hline 20000 & 3 & 0.00 & 0.09 & 0.00 & 0.00 & 0.00 & 0.17 \\
\hline 20000 & 7 & 0.00 & -0.49 & 0.00 & -0.08 & -0.03 & 0.32 \\
\hline 20000 & 14 & 0.00 & -0.16 & 0.00 & 0.00 & 0.00 & 0.43 \\
\hline 20000 & 28 & 0.00 & 0.30 & 0.00 & 0.00 & 0.01 & 0.44 \\
\hline
\end{tabular}


AVERAGE CONC. (ppm, blank corrected) FOR 18A FILTERED LEACHATES

\begin{tabular}{|c|c|c|c|c|c|c|c|}
\hline $\mathrm{SA} / \mathrm{V}$ & Duration & & & & & & \\
\hline$(m-1)$ & (days) & $\mathrm{CS}$ & $\mathrm{CU}$ & DY & EU & FE & GD \\
\hline 10 & 0.25 & 0.00 & 0.00 & 0.00 & 0.00 & 0.00 & 0.00 \\
\hline 10 & 0.5 & 0.01 & 0.00 & 0.00 & 0.00 & 0.00 & 0.00 \\
\hline 10 & 1 & 0.04 & 0.00 & 0.00 & 0.00 & 0.00 & 0.00 \\
\hline 10 & 3 & 0.11 & 0.00 & 0.00 & 0.00 & 0.00 & -0.01 \\
\hline 10 & 7 & 0.14 & 0.00 & 0.00 & 0.00 & 0.00 & -0.01 \\
\hline 10 & 14 & 0.15 & 0.00 & 0.00 & 0.00 & 0.00 & -0.01 \\
\hline 10 & 28 & 0.17 & 0.00 & 0.00 & 0.00 & 0.00 & -0.01 \\
\hline 10 & 56 & 0.20 & 0.00 & 0.00 & 0.00 & 0.00 & -0.01 \\
\hline 10 & 91 & 0.23 & 0.00 & 0.00 & 0.00 & 0.00 & -0.02 \\
\hline 10 & 182 & 0.24 & 0.00 & 0.00 & 0.00 & 0.00 & -0.01 \\
\hline 10 & 364 & 0.29 & 0.01 & 0.00 & 0.00 & 0.00 & -0.02 \\
\hline 530 & 0.25 & 0.15 & 0.00 & 0.00 & 0.00 & 0.00 & 0.00 \\
\hline 530 & 0.5 & 0.17 & 0.00 & 0.00 & 0.00 & 0.00 & 0.00 \\
\hline 530 & 1 & 0.20 & 0.00 & 0.00 & 0.00 & 0.00 & -0.01 \\
\hline 530 & 3 & 0.24 & 0.00 & 0.00 & 0.00 & 0.00 & -0.01 \\
\hline 530 & 7 & 0.25 & 0.00 & 0.00 & 0.00 & 0.00 & -0.02 \\
\hline 530 & 14 & 0.23 & 0.00 & 0.00 & 0.00 & 0.00 & -0.01 \\
\hline 530 & 28 & 0.20 & 0.00 & 0.00 & 0.00 & 0.00 & -0.02 \\
\hline 530 & 56 & 0.19 & 0.00 & 0.00 & 0.00 & 0.00 & -0.02 \\
\hline 530 & 91 & 0.22 & 0.00 & 0.00 & 0.00 & 0.00 & -0.01 \\
\hline 530 & 182 & 0.22 & 0.00 & 0.00 & 0.00 & 0.00 & -0.02 \\
\hline 2000 & 0.25 & 0.23 & 0.00 & 0.00 & 0.00 & 0.00 & 0.00 \\
\hline 2000 & 0.5 & 0.20 & 0.00 & 0.00 & 0.00 & 0.00 & 0.00 \\
\hline 2000 & 1 & 0.17 & 0.00 & 0.00 & 0.00 & 0.00 & -0.02 \\
\hline 2000 & 3 & 0.14 & 0.00 & 0.00 & 0.00 & 0.00 & -0.03 \\
\hline 2000 & 7 & 0.15 & 0.01 & 0.00 & 0.00 & 0.00 & -0.03 \\
\hline 2000 & 14 & 0.16 & 0.00 & 0.00 & 0.00 & 0.00 & 0.00 \\
\hline 2000 & 28 & 0.15 & 0.00 & 0.00 & 0.00 & 0.00 & -0.04 \\
\hline 2000 & 56 & 0.15 & 0.01 & 0.00 & 0.00 & 0.00 & -0.04 \\
\hline 2000 & 91 & 0.22 & 0.02 & 0.00 & 0.00 & 0.00 & -0.03 \\
\hline 2000 & 182 & 0.29 & 0.01 & 0.00 & 0.00 & 0.00 & -0.01 \\
\hline 20000 & 1 & 0.17 & 0.04 & 0.00 & 0.00 & 0.00 & 0.00 \\
\hline 20000 & 3 & $\overline{0.19}$ & 0.01 & 0.00 & 0.00 & 0.00 & -0.11 \\
\hline 20000 & 7 & 0.26 & 0.01 & -0.02 & -0.01 & -0.01 & -0.23 \\
\hline 20000 & 14 & 0.20 & 0.00 & 0.00 & 0.00 & 0.00 & -0.15 \\
\hline 20000 & 28 & 0.12 & 0.00 & 0.00 & 0.00 & 0.00 & -0.14 \\
\hline
\end{tabular}


AVERAGE CONC. (ppm, blank corrected) FOR 18A FILTERED LEACHATES

\begin{tabular}{|c|c|c|c|c|c|c|c|}
\hline $\mathrm{SA} / \mathrm{V}$ & Duration & & & & & & \\
\hline$(m-1)$ & (days) & $\overline{\mathbf{K}}$ & LA & LI & MG & MN & $\mathrm{MO}$ \\
\hline 10 & 0.25 & 0.00 & 0.00 & 0.06 & 0.00 & 0.00 & 0.01 \\
\hline 10 & 0.5 & 0.00 & 0.00 & 0.26 & 0.04 & 0.00 & 0.02 \\
\hline 10 & 1 & 0.00 & 0.00 & 0.49 & 0.10 & 0.00 & 0.05 \\
\hline 10 & 3 & 0.00 & 0.00 & 1.15 & 0.19 & 0.00 & 0.13 \\
\hline 10 & 7 & 0.00 & 0.00 & 1.63 & 0.04 & 0.00 & 0.17 \\
\hline 10 & 14 & 0.00 & 0.00 & 1.99 & 0.01 & 0.00 & 0.22 \\
\hline 10 & 28 & 0.00 & 0.00 & 2.46 & 0.01 & 0.00 & 0.26 \\
\hline 10 & 56 & 0.00 & 0.00 & 2.74 & 0.03 & 0.00 & 0.30 \\
\hline 10 & 91 & 0.00 & -0.01 & 2.95 & 0.01 & 0.01 & 0.32 \\
\hline 10 & 182 & 0.07 & 0.00 & 3.27 & 0.06 & 0.01 & 0.36 \\
\hline 10 & 364 & 0.00 & 0.01 & 3.88 & 0.08 & 0.01 & 0.43 \\
\hline 530 & 0.25 & -0.02 & 0.00 & 2.19 & 0.10 & 0.00 & 0.18 \\
\hline 530 & 0.5 & 0.03 & 0.00 & 2.87 & 0.07 & 0.00 & 0.27 \\
\hline 530 & 1 & 0.00 & 0.00 & 3.57 & 0.00 & 0.00 & 0.34 \\
\hline 530 & 3 & 0.00 & 0.00 & 5.36 & 0.01 & 0.00 & 0.55 \\
\hline 530 & 7 & 0.00 & 0.00 & 7.27 & 0.01 & 0.00 & 0.74 \\
\hline 530 & 14 & 0.00 & 0.00 & 8.23 & 0.03 & 0.00 & 0.84 \\
\hline 530 & 28 & 0.00 & 0.00 & 9.45 & 0.00 & 0.00 & 0.94 \\
\hline 530 & 56 & 0.00 & 0.00 & 10.66 & 0.01 & 0.00 & 1.04 \\
\hline 530 & 91 & 0.01 & 0.00 & 11.86 & 0.01 & 0.00 & 1.13 \\
\hline 530 & 182 & 0.00 & 0.00 & 13.00 & 0.01 & 0.00 & 1.16 \\
\hline 2000 & 0.25 & 0.02 & 0.00 & 4.79 & 0.02 & 0.00 & 0.39 \\
\hline 2000 & 0.5 & 0.06 & 0.00 & 5.60 & 0.00 & 0.00 & $\overline{0.46}$ \\
\hline 2000 & 1 & 0.00 & 0.00 & 8.39 & 0.00 & 0.00 & 0.78 \\
\hline 2000 & 3 & 0.00 & 0.00 & 12.66 & 0.00 & 0.00 & 1.24 \\
\hline 2000 & 7 & 0.00 & 0.00 & 15.70 & 0.01 & 0.00 & 1.48 \\
\hline 2000 & 14 & 0.00 & 0.00 & 17.26 & 0.02 & 0.00 & 1.63 \\
\hline 2000 & 28 & 0.00 & 0.00 & 20.03 & 0.00 & 0.01 & 1.77 \\
\hline 2000 & 56 & 0.00 & 0.00 & 22.47 & 0.04 & 0.01 & 1.92 \\
\hline 2000 & 91 & 0.00 & 0.01 & 24.80 & 0.10 & 0.02 & 2.11 \\
\hline 2000 & 182 & 0.00 & 0.01 & 29.03 & 0.05 & 0.01 & 2.46 \\
\hline 20000 & 1 & 0.00 & 0.00 & 43.55 & 0.13 & 0.00 & 3.62 \\
\hline 20000 & 3 & 0.00 & 0.00 & 60.66 & -0.01 & 0.00 & 5.47 \\
\hline 20000 & 7 & -0.98 & -0.02 & 70.49 & -0.15 & 0.01 & 6.24 \\
\hline 20000 & 14 & 0.00 & 0.00 & 80.13 & 0.00 & 0.00 & 6.59 \\
\hline 20000 & 28 & 0.00 & 0.01 & 87.76 & 0.04 & 0.04 & 8.13 \\
\hline
\end{tabular}


AVERAGE CONC. (ppm, blank corrected) FOR 18A FILTERED LEACHATES

\begin{tabular}{|c|c|c|c|c|c|c|c|}
\hline $\mathrm{SA} / \mathrm{V}$ & Duration & & & & & & \\
\hline$(m-1)$ & (days) & NA & ND & NI & $\mathbf{P}$ & PB & RU \\
\hline 10 & 0.25 & 0.25 & 0.00 & 0.00 & -0.01 & 0.00 & 0.00 \\
\hline 10 & 0.5 & 1.10 & 0.00 & 0.00 & 0.00 & 0.00 & 0.00 \\
\hline 10 & 1 & 2.07 & 0.00 & 0.00 & 0.00 & 0.00 & 0.00 \\
\hline 10 & 3 & 4.87 & 0.00 & 0.00 & -0.01 & 0.00 & 0.00 \\
\hline 10 & 7 & 7.06 & 0.00 & 0.00 & 0.00 & -0.02 & 0.00 \\
\hline 10 & 14 & 8.70 & 0.00 & 0.01 & 0.02 & -0.02 & 0.00 \\
\hline 10 & 28 & 10.05 & 0.00 & 0.00 & -0.01 & -0.03 & 0.00 \\
\hline 10 & 56 & 11.30 & 0.00 & 0.00 & 0.01 & -0.02 & 0.00 \\
\hline 10 & 91 & 12.70 & 0.00 & 0.00 & 0.01 & -0.04 & 0.00 \\
\hline 10 & 182 & 14.35 & 0.00 & 0.00 & 0.05 & -0.01 & 0.00 \\
\hline 10 & 364 & 15.78 & 0.01 & 0.02 & 0.08 & 0.02 & 0.00 \\
\hline 530 & 0.25 & 8.88 & 0.00 & 0.00 & 0.01 & -0.02 & 0.00 \\
\hline 530 & 0.5 & 11.45 & 0.00 & 0.00 & 0.02 & 0.00 & 0.01 \\
\hline 530 & 1 & 14.83 & 0.00 & 0.00 & 0.02 & -0.04 & 0.00 \\
\hline 530 & 3 & 22.59 & 0.00 & 0.00 & 0.04 & -0.04 & 0.00 \\
\hline 530 & 7 & 30.82 & 0.00 & 0.00 & 0.06 & -0.05 & 0.00 \\
\hline 530 & 14 & 35.20 & 0.00 & 0.01 & 0.08 & -0.07 & 0.02 \\
\hline & ff & 39.05 & 0.00 & 0.00 & 0.07 & -0.01 & 0.00 \\
\hline 530 & 56 & 41.95 & 0.00 & 0.00 & 0.08 & -0.05 & 0.00 \\
\hline 530 & 91 & 47.48 & 0.00 & 0.00 & 0.09 & -0.05 & 0.00 \\
\hline 530 & 182 & 49.44 & 0.00 & 0.00 & 0.09 & 0.00 & 0.00 \\
\hline 2000 & 0.25 & 18.18 & 0.00 & 0.00 & 0.05 & 0.02 & 0.00 \\
\hline 2000 & 0.5 & 21.75 & 0.00 & 0.00 & 0.03 & 0.00 & 0.00 \\
\hline 2000 & 1 & 31.86 & 0.00 & 0.00 & 0.06 & -0.05 & 0.00 \\
\hline 2000 & 3 & 50.21 & 0.00 & 0.00 & 0.06 & -0.09 & 0.00 \\
\hline 2000 & 7 & 61.37 & 0.00 & 0.01 & 0.11 & -0.08 & 0.00 \\
\hline 2000 & 14 & 68.10 & 0.00 & 0.01 & 0.13 & -0.09 & 0.02 \\
\hline 2000 & 28 & 74.64 & 0.00 & 0.01 & 0.08 & -0.01 & 0.00 \\
\hline 2000 & 56 & 81.33 & 0.00 & 0.01 & 0.16 & -0.09 & 0.00 \\
\hline 2000 & 91 & 91.24 & 0.01 & 0.05 & 0.18 & -0.11 & 0.00 \\
\hline 2000 & 182 & 100.95 & 0.00 & 0.02 & 0.22 & 0.02 & 0.01 \\
\hline 20000 & 1 & 146.32 & 0.00 & 0.01 & 0.23 & 0.01 & 0.00 \\
\hline 20000 & 3 & 217.74 & 0.00 & -0.01 & 0.22 & -0.24 & 0.00 \\
\hline 20000 & 7 & 253.41 & -0.07 & 0.00 & 0.25 & -0.42 & 0.00 \\
\hline 20000 & 14 & 281.97 & 0.00 & 0.00 & 0.39 & -0.28 & 0.00 \\
\hline 20000 & 28 & 310.18 & 0.00 & 0.00 & 0.33 & 0.00 & 0.01 \\
\hline
\end{tabular}


AVERAGE CONC. (ppm, blank corrected) FOR 18A FILTERED LEACHATES

\begin{tabular}{|c|c|c|c|c|c|c|c|}
\hline SA/V & Duration & & & & & & \\
\hline$(m-1)$ & (days) & SB & SI & SR & TE & TI & V \\
\hline 10 & 0.25 & 0.00 & 0.75 & 0.00 & 0.00 & 0.00 & 0.00 \\
\hline 10 & 0.5 & 0.00 & 3.57 & 0.01 & 0.00 & 0.00 & 0.00 \\
\hline 10 & 1 & 0.00 & 6.56 & 0.01 & 0.00 & 0.00 & 0.00 \\
\hline 10 & 3 & 0.01 & 14.44 & 0.03 & 0.00 & 0.00 & 0.00 \\
\hline 10 & 7 & 0.01 & 19.44 & 0.04 & -0.01 & 0.00 & 0.00 \\
\hline 10 & 14 & 0.00 & 23.29 & 0.04 & -0.01 & 0.00 & 0.00 \\
\hline 10 & 28 & 0.00 & 26.89 & 0.04 & -0.01 & 0.00 & 0.00 \\
\hline 10 & 56 & 0.01 & 29.58 & 0.04 & 0.00 & 0.00 & 0.00 \\
\hline 10 & 91 & 0.00 & 32.03 & 0.04 & -0.03 & 0.00 & 0.00 \\
\hline 10 & 182 & 0.00 & 35.43 & 0.04 & -0.02 & 0.00 & 0.00 \\
\hline 10 & 364 & 0.01 & 40.33 & 0.04 & -0.01 & 0.00 & 0.00 \\
\hline 530 & 0.25 & 0.00 & 22.69 & 0.01 & 0.04 & 0.00 & 0.00 \\
\hline 530 & 0.5 & -0.01 & 27.65 & 0.01 & 0.00 & 0.00 & 0.00 \\
\hline 530 & 1 & 0.00 & 32.20 & 0.00 & -0.02 & 0.00 & 0.00 \\
\hline 530 & 3 & 0.02 & 46.29 & 0.00 & -0.01 & 0.00 & 0.00 \\
\hline 530 & 7 & 0.02 & 56.37 & 0.00 & -0.02 & 0.00 & 0.00 \\
\hline 530 & 14 & 0.02 & 64.77 & 0.00 & -0.02 & 0.00 & 0.00 \\
\hline 530 & 28 & 0.02 & 68.06 & 0.01 & -0.04 & 0.00 & 0.00 \\
\hline 530 & 56 & 0.01 & 69.43 & 0.00 & 0.01 & 0.00 & 0.00 \\
\hline 530 & 91 & 0.03 & 76.68 & 0.01 & 0.02 & 0.00 & 0.00 \\
\hline 530 & 182 & 0.03 & 81.76 & 0.01 & -0.02 & 0.00 & 0.00 \\
\hline 2000 & 0.25 & 0.01 & 36.91 & 0.00 & 0.13 & 0.00 & 0.00 \\
\hline 2000 & 0.5 & 0.00 & 43.06 & 0.00 & 0.02 & 0.00 & 0.00 \\
\hline 2000 & 1 & 0.01 & 56.43 & 0.00 & 0.02 & 0.00 & 0.00 \\
\hline 2000 & 3 & 0.01 & 79.79 & 0.00 & -0.01 & 0.00 & 0.00 \\
\hline 2000 & 7 & 0.02 & 91.37 & 0.00 & 0.02 & 0.00 & $\overline{0.00}$ \\
\hline 2000 & 14 & 0.03 & 99.67 & 0.00 & 0.02 & 0.00 & 0.00 \\
\hline 2000 & 28 & 0.04 & 105.28 & 0.00 & -0.01 & 0.00 & $\overline{0.00}$ \\
\hline 2000 & 56 & 0.02 & 108.33 & 0.00 & 0.03 & 0.00 & 0.00 \\
\hline 2000 & 91 & 0.02 & 118.65 & 0.01 & -0.01 & 0.00 & 0.00 \\
\hline 2000 & 182 & 0.06 & 137.00 & 0.01 & -0.04 & 0.00 & 0.01 \\
\hline 20000 & 1 & 0.04 & 183.97 & 0.00 & 0.37 & 0.00 & 0.00 \\
\hline 20000 & 3 & 0.00 & 214.14 & 0.00 & 0.17 & 0.00 & 0.00 \\
\hline 20000 & 7 & -0.03 & 235.73 & -0.01 & 0.58 & -0.01 & -0.02 \\
\hline 20000 & 14 & 0.03 & 265.17 & 0.00 & 0.44 & 0.00 & 0.00 \\
\hline 20000 & 28 & 0.12 & 287.07 & 0.00 & 0.22 & 0.00 & 0.00 \\
\hline
\end{tabular}


AVERAGE CONC. (ppm, blank corrected) FOR 18A FILTERED LEACHATES

\begin{tabular}{|c|c|c|c|c|}
\hline $\mathrm{SA} / \mathrm{V}$ & Duration & & & \\
\hline$(m-1)$ & (days) & $\mathbf{Y}$ & $\mathbf{Z N}$ & ZR \\
\hline 10 & 0.25 & 0.00 & -0.02 & 0.00 \\
\hline 10 & 0.5 & 0.00 & 0.00 & 0.00 \\
\hline 10 & 1 & 0.00 & 0.00 & 0.00 \\
\hline 10 & 3 & 0.00 & 0.00 & 0.00 \\
\hline 10 & 7 & 0.00 & 0.00 & 0.00 \\
\hline 10 & 14 & 0.00 & 0.00 & 0.00 \\
\hline 10 & 28 & 0.00 & 0.00 & -0.01 \\
\hline 10 & 56 & 0.00 & -0.03 & 0.00 \\
\hline 10 & 91 & 0.00 & 0.00 & 0.01 \\
\hline 10 & 182 & 0.00 & 0.00 & 0.00 \\
\hline 10 & 364 & 0.00 & 0.00 & 0.00 \\
\hline 530 & 0.25 & 0.00 & -0.34 & 0.00 \\
\hline 530 & 0.5 & 0.00 & -0.09 & 0.00 \\
\hline 530 & 1 & 0.00 & -0.18 & 0.00 \\
\hline 530 & 3 & 0.00 & -0.02 & 0.00 \\
\hline 530 & 7 & 0.00 & -0.06 & 0.00 \\
\hline 530 & 14 & 0.00 & 0.26 & 0.01 \\
\hline 530 & 28 & 0.00 & 0.17 & 0.00 \\
\hline 530 & 56 & 0.00 & 0.17 & 0.00 \\
\hline 530 & 91 & 0.00 & 0.04 & 0.01 \\
\hline 530 & 182 & 0.00 & 0.29 & 0.00 \\
\hline 2000 & 0.25 & 0.00 & -0.08 & 0.00 \\
\hline 2000 & 0.5 & 0.00 & -0.10 & 0.00 \\
\hline 2000 & 1 & 0.00 & 0.28 & 0.00 \\
\hline 2000 & 3 & 0.00 & 0.08 & 0.00 \\
\hline 2000 & 7 & 0.00 & 0.18 & 0.01 \\
\hline 2000 & 14 & 0.00 & 0.33 & 0.01 \\
\hline 2000 & 28 & 0.00 & 0.16 & 0.01 \\
\hline 2000 & 56 & 0.00 & 0.50 & 0.01 \\
\hline 2000 & 91 & 0.00 & 0.78 & 0.03 \\
\hline 2000 & 182 & 0.00 & 0.54 & 0.01 \\
\hline 20000 & 1 & 0.00 & 0.30 & 0.00 \\
\hline 20000 & 3 & 0.00 & 1.47 & $\overline{0.02}$ \\
\hline 20000 & 7 & -0.01 & -0.59 & 0.04 \\
\hline 20000 & 14 & 0.00 & 0.18 & 0.03 \\
\hline 20000 & 28 & 0.00 & 1.15 & 0.04 \\
\hline
\end{tabular}



AVERAGE CONC. (ppm, blank corrected) FOR 0.45u FILTERED LEACHATES

\begin{tabular}{|c|c|c|c|c|c|c|c|}
\hline $\mathrm{SA} / \mathrm{V}$ & Duration & & & & & & \\
\hline$(m-1)$ & (days) & pH final & $\mathrm{pH}$ in & $\overline{A L}$ & AS & B & $\overline{\mathrm{BA}}$ \\
\hline 10 & 0.25 & 7.36 & 5.58 & 0.08 & 0.00 & 0.10 & 0.00 \\
\hline 10 & 0.5 & 9.02 & 5.88 & 0.34 & 0.00 & 0.48 & 0.00 \\
\hline 10 & 1 & 9.51 & 5.67 & 0.64 & -0.01 & 0.91 & 0.01 \\
\hline 10 & 3 & 9.67 & 5.37 & 1.37 & 0.00 & 2.05 & 0.01 \\
\hline 10 & 7 & 9.52 & 5.74 & 1.66 & 0.01 & 2.89 & 0.01 \\
\hline 10 & 14 & 9.71 & 5.74 & 1.86 & -0.01 & 3.53 & 0.01 \\
\hline 10 & 28 & 9.32 & 6.01 & 2.04 & 0.01 & 4.33 & 0.01 \\
\hline 10 & 56 & 9.44 & 6.01 & 2.11 & 0.02 & 4.85 & 0.01 \\
\hline 10 & 91 & 9.33 & 6.01 & 2.09 & 0.00 & 5.29 & 0.01 \\
\hline 10 & 182 & 9.44 & 6.01 & 2.13 & -0.01 & 5.89 & 0.01 \\
\hline 10 & 364 & 8.72 & 6.00 & 1.89 & 0.01 & 6.83 & 0.01 \\
\hline 530 & 0.25 & 9.90 & 5.58 & 1.91 & -0.01 & 3.30 & 0.01 \\
\hline 530 & 0.5 & 9.96 & 5.88 & 2.03 & 0.00 & 4.22 & 0.01 \\
\hline 530 & 1 & 9.95 & 5.74 & 2.33 & -0.01 & 5.68 & 0.02 \\
\hline 530 & 3 & 9.99 & 5.51 & 3.08 & 0.03 & 8.85 & 0.03 \\
\hline 530 & 7 & 10.09 & 5.64 & 4.05 & 0.02 & 11.92 & 0.04 \\
\hline 530 & 14 & 9.99 & 5.69 & 4.62 & 0.02 & 14.12 & 0.05 \\
\hline 530 & 28 & 10.21 & 5.55 & 5.65 & 0.01 & 15.47 & 0.08 \\
\hline 530 & 56 & 10.10 & 5.61 & 4.82 & 0.02 & 17.03 & 0.06 \\
\hline 530 & 91 & 9.88 & 5.61 & 5.42 & 0.03 & 18.22 & $\overline{0.06}$ \\
\hline 530 & 182 & 9.96 & 5.61 & 5.91 & 0.01 & 19.77 & 0.07 \\
\hline 2000 & 0.25 & 10.15 & 5.58 & 2.28 & 0.01 & 6.13 & 0.01 \\
\hline 2000 & 0.5 & 10.18 & 5.88 & 2.42 & 0.01 & 7.80 & 0.01 \\
\hline 2000 & 1 & 10.04 & 5.69 & 2.84 & $\overline{0.02}$ & 12.60 & 0.01 \\
\hline 2000 & 3 & 10.31 & 5.51 & 3.78 & 0.00 & 20.03 & 0.02 \\
\hline 2000 & 7 & 10.38 & 5.74 & 5.54 & 0.04 & 25.23 & $\overline{0.04}$ \\
\hline 2000 & 14 & 10.28 & 5.69 & 6.46 & 0.04 & 28.19 & $\overline{0.04}$ \\
\hline 2000 & 28 & 10.55 & 5.55 & 7.53 & 0.02 & 30.78 & 0.07 \\
\hline 2000 & 56 & 10.42 & 5.61 & 7.85 & 0.04 & 33.58 & 0.07 \\
\hline 2000 & 91 & 10.27 & 5.61 & 10.27 & 0.06 & 36.53 & 0.09 \\
\hline 2000 & 182 & 10.35 & 5.61 & 12.07 & 0.03 & 41.20 & 0.13 \\
\hline 20000 & 1 & 10.78 & 5.58 & 3.33 & 0.02 & 63.30 & 0.02 \\
\hline 20000 & 3 & 10.89 & 5.51 & 6.82 & -0.05 & 98.25 & 0.03 \\
\hline 20000 & 7 & 10.89 & 5.64 & 11.69 & 0.08 & 113.90 & 0.05 \\
\hline 20000 & 14 & 10.93 & 5.64 & 16.03 & -0.02 & 129.41 & 0.06 \\
\hline 20000 & 28 & 11.20 & 5.55 & 0.52 & 0.00 & 149.00 & 0.00 \\
\hline
\end{tabular}


AVERAGE CONC. (ppm, blank corrected) FOR 0.45u FILTERED LEACHATES

\begin{tabular}{|c|c|c|c|c|c|c|c|}
\hline $\mathrm{SA} / \mathrm{V}$ & Duration & & & & & & \\
\hline$(m-1)$ & (days) & $\mathrm{BE}$ & $\overline{\mathbf{C A}}$ & CD & $\overline{\mathrm{CE}}$ & $\mathrm{CO}$ & $\overline{C R}$ \\
\hline 10 & 0.25 & 0.00 & 0.03 & 0.00 & 0.00 & 0.00 & 0.00 \\
\hline 10 & 0.5 & 0.00 & 0.26 & 0.00 & 0.00 & 0.00 & 0.00 \\
\hline 10 & 1 & 0.00 & 0.47 & 0.00 & 0.00 & 0.00 & 0.00 \\
\hline 10 & 3 & 0.00 & 1.08 & 0.00 & 0.00 & 0.00 & 0.00 \\
\hline 10 & 7 & 0.00 & 1.29 & 0.00 & 0.00 & 0.00 & 0.00 \\
\hline 10 & 14 & 0.00 & 1.35 & 0.00 & 0.00 & 0.00 & 0.00 \\
\hline 10 & 28 & 0.00 & 1.49 & 0.00 & 0.00 & 0.00 & 0.00 \\
\hline 10 & 56 & 0.00 & 1.52 & 0.00 & 0.00 & 0.00 & 0.01 \\
\hline 10 & 91 & 0.00 & 1.47 & 0.00 & 0.00 & 0.00 & 0.01 \\
\hline 10 & 182 & 0.00 & 1.79 & 0.00 & 0.00 & 0.00 & 0.01 \\
\hline 10 & 364 & 0.00 & 1.83 & 0.00 & 0.01 & 0.00 & 0.01 \\
\hline 530 & 0.25 & 0.00 & 0.94 & 0.00 & 0.00 & 0.00 & 0.01 \\
\hline 530 & 0.5 & 0.00 & 0.90 & 0.00 & 0.01 & 0.00 & 0.02 \\
\hline 530 & 1 & 0.00 & 0.95 & 0.00 & 0.02 & 0.00 & $0 . \overline{06}$ \\
\hline 530 & 3 & 0.00 & 0.89 & 0.00 & 0.05 & 0.01 & 0.05 \\
\hline 530 & 7 & 0.00 & 1.41 & 0.00 & 0.04 & 0.00 & 0.07 \\
\hline 530 & 14 & 0.00 & 1.92 & 0.67 & 0.06 & 0.00 & 0.07 \\
\hline 530 & 28 & 0.00 & 2.69 & 0.00 & 0.09 & 0.00 & 0.13 \\
\hline 530 & 56 & 0.00 & 2.26 & 0.00 & 0.08 & 0.00 & 0.11 \\
\hline 530 & 91 & 0.00 & 2.55 & 0.00 & 0.09 & 0.01 & 0.13 \\
\hline 530 & 182 & 0.00 & 3.14 & 0.00 & 0.08 & 0.00 & 0.18 \\
\hline 2000 & 0.25 & 0.00 & 0.29 & 0.00 & 0.00 & 0.00 & 0.01 \\
\hline 2000 & 0.5 & 0.00 & 0.26 & 0.00 & 0.00 & 0.00 & 0.01 \\
\hline 2000 & 1 & 0.00 & 0.33 & 0.00 & 0.03 & 0.01 & 0.04 \\
\hline 2000 & 3 & 0.00 & 0.90 & 0.00 & 0.03 & 0.00 & 0.08 \\
\hline 2000 & 7 & 0.00 & 1.64 & 0.00 & 0.11 & 0.02 & 0.15 \\
\hline 2000 & 14 & 0.00 & 2.03 & 1.64 & 0.14 & 0.01 & 0.17 \\
\hline 2000 & 28 & 0.00 & 2.72 & 0.00 & 0.14 & 0.01 & 0.20 \\
\hline 2000 & 56 & 0.00 & 3.11 & 0.00 & 0.25 & 0.02 & 0.31 \\
\hline 2000 & 91 & 0.00 & 4.43 & 0.00 & 0.34 & 0.02 & 0.42 \\
\hline 2000 & 182 & 0.00 & 6.06 & 0.00 & 0.52 & 0.02 & 0.60 \\
\hline 20000 & 1 & 0.00 & 0.88 & 0.01 & 0.00 & 0.00 & 0.10 \\
\hline 20000 & 3 & 0.00 & 1.96 & 0.00 & 0.02 & 0.09 & 0.39 \\
\hline 20000 & 7 & 0.00 & 2.95 & 0.00 & 0.32 & 0.11 & 0.73 \\
\hline 20000 & 14 & 0.00 & 3.27 & 0.01 & 0.38 & 0.12 & 1.16 \\
\hline 20000 & 28 & 0.00 & 0.24 & 0.00 & 0.00 & 0.01 & 0.49 \\
\hline
\end{tabular}


AVERAGE CONC. (ppm, blank corrected) FOR 0.45u FILTERED LEACHATES

\begin{tabular}{|c|c|c|c|c|c|c|c|}
\hline $\mathrm{SA} / \mathrm{V}$ & Duration & & & & & & \\
\hline$(m-1)$ & (days) & $\mathrm{CS}$ & $\mathrm{CU}$ & DY & $\overline{E U}$ & $\overline{F E}$ & GD \\
\hline 10 & 0.25 & 0.00 & 0.00 & 0.00 & 0.00 & 0.00 & 0.00 \\
\hline 10 & 0.5 & 0.01 & 0.00 & 0.00 & 0.00 & 0.00 & 0.00 \\
\hline 10 & 1 & 0.04 & 0.00 & 0.00 & 0.00 & 0.00 & 0.00 \\
\hline 10 & 3 & 0.11 & 0.00 & 0.00 & 0.00 & 0.00 & -0.01 \\
\hline 10 & 7 & 0.14 & 0.00 & 0.00 & 0.00 & 0.00 & -0.01 \\
\hline 10 & 14 & 0.15 & 0.00 & 0.00 & 0.00 & 0.00 & -0.01 \\
\hline 10 & 28 & 0.17 & 0.00 & 0.00 & 0.00 & 0.00 & -0.01 \\
\hline 10 & 56 & 0.20 & 0.00 & 0.00 & 0.00 & 0.00 & -0.01 \\
\hline 10 & 91 & 0.23 & 0.00 & 0.00 & 0.00 & 0.00 & -0.02 \\
\hline 10 & 182 & 0.24 & 0.00 & 0.00 & 0.00 & 0.00 & -0.01 \\
\hline 10 & 364 & 0.29 & 0.01 & 0.00 & 0.00 & 0.00 & -0.02 \\
\hline 530 & 0.25 & 0.17 & 0.03 & 0.00 & 0.00 & 0.00 & 0.00 \\
\hline 530 & 0.5 & 0.19 & 0.05 & 0.00 & 0.00 & 0.00 & 0.00 \\
\hline 530 & 1 & 0.21 & 0.07 & 0.00 & 0.00 & 0.00 & -0.02 \\
\hline 530 & 3 & 0.30 & 0.09 & 0.00 & 0.00 & 0.00 & -0.01 \\
\hline 530 & 7 & 0.40 & 0.14 & 0.00 & 0.00 & 0.00 & $\overline{-0.04}$ \\
\hline 530 & 14 & 0.40 & 0.18 & 0.00 & 0.00 & 0.00 & 0.00 \\
\hline 530 & 28 & 0.40 & 0.26 & 0.00 & 0.00 & 0.00 & -0.06 \\
\hline 530 & 56 & 0.36 & 0.23 & 0.00 & 0.00 & 0.00 & -0.03 \\
\hline 530 & 91 & 0.44 & 0.26 & 0.00 & 0.00 & 0.00 & -0.03 \\
\hline 530 & 182 & 0.49 & \begin{tabular}{l|l|}
0.30 \\
\end{tabular} & 0.00 & 0.00 & 0.00 & -0.04 \\
\hline 2000 & 0.25 & 0.25 & 0.04 & 0.00 & 0.00 & 0.00 & 0.00 \\
\hline 2000 & 0.5 & 0.23 & 0.04 & 0.00 & 0.00 & 0.00 & 0.00 \\
\hline 2000 & 1 & 0.21 & \begin{tabular}{|c|}
0.07 \\
\end{tabular} & 0.00 & 0.00 & 0.00 & -0.02 \\
\hline 2000 & 3 & 0.23 & 0.17 & 0.00 & 0.00 & 0.00 & -0.05 \\
\hline 2000 & 7 & 0.33 & 0.40 & 0.00 & 0.00 & 0.00 & -0.03 \\
\hline 2000 & 14 & 0.41 & 0.46 & 0.00 & 0.00 & 0.00 & -0.04 \\
\hline 2000 & 28 & 0.39 & 0.63 & 0.00 & 0.00 & 0.00 & -0.09 \\
\hline 2000 & 56 & 0.44 & 0.67 & 0.00 & 0.00 & 0.00 & -0.06 \\
\hline 2000 & 91 & 0.65 & 0.84 & 0.00 & 0.00 & 0.00 & -0.05 \\
\hline 2000 & 182 & 0.87 & 0.89 & 0.00 & 0.00 & 0.00 & -0.08 \\
\hline 20000 & 1 & 0.24 & 0.81 & 0.00 & 0.00 & 0.00 & 0.00 \\
\hline 20000 & 3 & 0.42 & 2.26 & 0.00 & 0.00 & 0.00 & -0.22 \\
\hline 20000 & 7 & 0.66 & 4.05 & -0.01 & 0.00 & 0.00 & -0.17 \\
\hline 20000 & 14 & 0.54 & 4.89 & 0.00 & 0.00 & 0.00 & -0.35 \\
\hline 20000 & 28 & 0.14 & 0.01 & 0.01 & 0.00 & 0.00 & -0.09 \\
\hline
\end{tabular}


AVERAGE CONC. (ppm, blank corrected) FOR 0.45u FILTERED LEACHATES

\begin{tabular}{|c|c|c|c|c|c|c|c|}
\hline $\mathrm{SA} / \mathrm{V}$ & Duration & & & & & & \\
\hline$(m-1)$ & (days) & $\mathbf{K}$ & LA & LI & MG & $\mathrm{MN}$ & $\mathrm{MO}$ \\
\hline 10 & 0.25 & 0.00 & 0.00 & 0.06 & 0.00 & 0.00 & 0.01 \\
\hline 10 & 0.5 & 0.00 & 0.00 & 0.26 & 0.04 & 0.00 & 0.02 \\
\hline 10 & 1 & 0.00 & 0.00 & 0.49 & 0.10 & 0.00 & 0.05 \\
\hline 10 & 3 & 0.00 & 0.00 & 1.15 & 0.19 & 0.00 & 0.13 \\
\hline 10 & 7 & 0.00 & 0.00 & 1.63 & 0.04 & 0.00 & 0.17 \\
\hline 10 & 14 & 0.00 & 0.00 & 1.99 & 0.01 & 0.00 & 0.22 \\
\hline 10 & 28 & 0.00 & 0.00 & 2.46 & 0.01 & 0.00 & 0.26 \\
\hline 10 & 56 & 0.00 & 0.00 & 2.74 & 0.03 & 0.00 & 0.30 \\
\hline 10 & 91 & 0.00 & -0.01 & 2.95 & 0.01 & 0.01 & 0.32 \\
\hline 10 & 182 & 0.07 & 0.00 & 3.27 & 0.06 & 0.01 & 0.36 \\
\hline 10 & 364 & 0.00 & 0.01 & 3.88 & 0.08 & 0.01 & 0.43 \\
\hline 530 & 0.25 & 0.00 & 0.07 & 2.23 & 0.18 & 0.02 & 0.20 \\
\hline 530 & 0.5 & 0.00 & 0.11 & 2.88 & 0.18 & 0.03 & 0.26 \\
\hline 530 & 1 & 0.00 & 0.16 & 3.56 & 0.26 & 0.06 & 0.34 \\
\hline 530 & 3 & 0.00 & 0.14 & 5.36 & 0.63 & 0.10 & 0.55 \\
\hline 530 & 7 & 0.00 & 0.15 & 7.37 & 1.06 & 0.17 & 0.73 \\
\hline 530 & 14 & -0.14 & 0.18 & 8.42 & 1.37 & 0.22 & 0.87 \\
\hline 530 & 28 & 0.00 & 0.28 & 9.79 & 1.65 & 0.29 & 0.98 \\
\hline 530 & 56 & 0.03 & 0.22 & 11.00 & 1.33 & 0.23 & 1.02 \\
\hline 530 & 91 & 0.00 & 0.22 & 12.03 & 1.50 & 0.25 & 1.12 \\
\hline 530 & 182 & 0.00 & 0.28 & 13.40 & 1.60 & 0.28 & 1.18 \\
\hline 2000 & 0.25 & 0.02 & 0.05 & 4.80 & 0.07 & 0.01 & 0.38 \\
\hline 2000 & 0.5 & 0.01 & 0.06 & 5.64 & 0.07 & 0.02 & 0.48 \\
\hline 2000 & 1 & 0.00 & 0.10 & 8.47 & 0.26 & 0.04 & 0.78 \\
\hline 2000 & 3 & 0.00 & 0.19 & 12.82 & 1.57 & 0.20 & 1.20 \\
\hline 2000 & 7 & 0.00 & 0.28 & 16.17 & 2.57 & 0.40 & 1.44 \\
\hline 2000 & 14 & 0.00 & 0.31 & 18.13 & 3.02 & 0.48 & 1.65 \\
\hline 2000 & 28 & 0.00 & 0.37 & 21.46 & 3.43 & 0.57 & 1.81 \\
\hline 2000 & 56 & 0.00 & 0.52 & 23.73 & 3.36 & 0.58 & 1.90 \\
\hline 2000 & 91 & 0.00 & 0.71 & 26.63 & 4.25 & 0.72 & 2.06 \\
\hline 2000 & 182 & 0.00 & 1.13 & 31.13 & 4.24 & 0.74 & 2.24 \\
\hline 20000 & 1 & 0.02 & 0.22 & 44.61 & 3.91 & 0.48 & 3.61 \\
\hline 20000 & 3 & 0.00 & 0.43 & 65.20 & 9.30 & 1.55 & 5.42 \\
\hline 20000 & 7 & -0.41 & 0.71 & 78.75 & 13.03 & 2.29 & 6.20 \\
\hline 20000 & 14 & 0.00 & 1.09 & 92.37 & 14.42 & 2.69 & 6.77 \\
\hline 20000 & 28 & 0.00 & 0.10 & 88.14 & 0.08 & 0.01 & 8.19 \\
\hline
\end{tabular}


AVERAGE CONC. (ppm, blank corrected) FOR 0.45u FILTERED LEACHATES

\begin{tabular}{|c|c|c|c|c|c|c|c|}
\hline $\mathrm{SA} / \mathrm{V}$ & Duration & & & & & & \\
\hline$(m-1)$ & (days) & NA & ND & NI & $\overline{\mathbf{P}}$ & PB & RU \\
\hline 10 & 0.25 & 0.25 & 0.00 & 0.00 & -0.01 & 0.00 & 0.00 \\
\hline 10 & 0.5 & 1.10 & 0.00 & 0.00 & 0.00 & 0.00 & 0.00 \\
\hline 10 & 1 & 2.07 & 0.00 & 0.00 & 0.00 & 0.00 & 0.00 \\
\hline 10 & 3 & 4.87 & 0.00 & 0.00 & -0.01 & 0.00 & 0.00 \\
\hline 10 & 7 & 7.06 & 0.00 & 0.00 & 0.00 & -0.02 & 0.00 \\
\hline 10 & 14 & 8.70 & 0.00 & 0.01 & 0.02 & -0.02 & 0.00 \\
\hline 10 & 28 & 10.05 & 0.00 & 0.00 & -0.01 & -0.03 & 0.00 \\
\hline 10 & 56 & 11.30 & 0.00 & 0.00 & 0.01 & -0.02 & 0.00 \\
\hline 10 & 91 & 12.70 & 0.00 & 0.00 & 0.01 & -0.04 & 0.00 \\
\hline 10 & 182 & 14.35 & 0.00 & 0.00 & 0.05 & -0.01 & 0.00 \\
\hline 10 & 364 & 15.78 & 0.01 & 0.02 & 0.08 & 0.02 & 0.00 \\
\hline 530 & 0.25 & 8.90 & 0.03 & 0.06 & 0.01 & 0.02 & 0.00 \\
\hline 530 & 0.5 & 11.26 & 0.06 & 0.08 & 0.00 & 0.02 & 0.01 \\
\hline 530 & 1 & 14.80 & 0.10 & 0.17 & 0.02 & -0.04 & 0.00 \\
\hline 530 & 3 & 22.58 & 0.10 & 0.24 & 0.04 & -0.04 & 0.00 \\
\hline 530 & 7 & 31.00 & 0.11 & 0.38 & 0.02 & -0.05 & 0.00 \\
\hline 530 & 14 & 35.39 & 0.14 & 0.50 & 0.08 & -0.05 & 1.43 \\
\hline 530 & 28 & 39.67 & 0.23 & 0.66 & 0.05 & -0.01 & 0.06 \\
\hline 530 & 56 & 42.84 & 0.18 & 0.59 & 0.07 & -0.06 & 0.00 \\
\hline 530 & 91 & 47.63 & 0.19 & 0.67 & 0.09 & -0.06 & 0.00 \\
\hline 530 & 182 & 50.57 & 0.22 & 0.78 & 0.08 & -0.01 & 0.06 \\
\hline 2000 & 0.25 & 18.10 & 0.01 & 0.04 & 0.03 & 0.02 & 0.00 \\
\hline 2000 & 0.5 & 21.66 & 0.02 & 0.05 & 0.00 & 0.03 & 0.00 \\
\hline 2000 & 1 & 32.23 & 0.08 & 0.16 & 0.06 & -0.05 & 0.00 \\
\hline 2000 & 3 & 50.40 & 0.12 & 0.51 & 0.05 & -0.10 & 0.00 \\
\hline 2000 & 7 & 61.93 & 0.22 & 1.07 & 0.09 & -0.08 & 0.00 \\
\hline 2000 & 14 & 69.71 & 0.26 & 1.29 & 0.10 & -0.08 & 2.83 \\
\hline 2000 & 28 & 77.67 & 0.31 & $1 . \overline{43}$ & 0.04 & -0.01 & 0.08 \\
\hline 2000 & 56 & 83.40 & 0.47 & 1.75 & 0.14 & -0.08 & 0.00 \\
\hline 2000 & 91 & 95.63 & 0.66 & 2.27 & 0.15 & -0.08 & 0.00 \\
\hline 2000 & 182 & 102.33 & 1.05 & 2.44 & 0.16 & 0.01 & 0.18 \\
\hline 20000 & 1 & 147.81 & 0.08 & 1.40 & 0.18 & 0.07 & 0.05 \\
\hline 20000 & 3 & 223.27 & 0.23 & 4.27 & 0.22 & -0.32 & 0.00 \\
\hline 20000 & 7 & 262.73 & 0.63 & 6.52 & 0.27 & -0.33 & 0.00 \\
\hline 20000 & 14 & 295.73 & 0.92 & 7.48 & 0.29 & -0.47 & 0.64 \\
\hline 20000 & 28 & 312.59 & 0.00 & 0.00 & 0.25 & 0.00 & 0.01 \\
\hline
\end{tabular}


AVERAGE CONC. (ppm, blank corrected) FOR 0.45u FILTERED LEACHATES

\begin{tabular}{|c|c|c|c|c|c|c|c|}
\hline SA/V & Duration & & & & & & \\
\hline$(m-1)$ & (days) & SB & SI & $\overline{\text { SR }}$ & TE & TI & $\mathrm{V}$ \\
\hline 10 & 0.25 & 0.00 & 0.75 & 0.00 & 0.00 & 0.00 & 0.00 \\
\hline 10 & 0.5 & 0.00 & 3.57 & 0.01 & 0.00 & 0.00 & 0.00 \\
\hline 10 & 1 & 0.00 & 6.56 & 0.01 & 0.00 & 0.00 & 0.00 \\
\hline 10 & 3 & 0.01 & 14.44 & 0.03 & 0.00 & 0.00 & 0.00 \\
\hline 10 & 7 & 0.01 & 19.44 & 0.04 & -0.01 & 0.00 & 0.00 \\
\hline 10 & 14 & 0.00 & 23.29 & 0.04 & -0.01 & 0.00 & 0.00 \\
\hline 10 & 28 & 0.00 & 26.89 & 0.04 & -0.01 & 0.00 & 0.00 \\
\hline 10 & 56 & 0.01 & 29.58 & 0.04 & 0.00 & 0.00 & 0.00 \\
\hline 10 & 91 & 0.00 & 32.03 & 0.04 & -0.03 & 0.00 & 0.00 \\
\hline 10 & 182 & 0.00 & 35.43 & 0.04 & -0.02 & 0.00 & 0.00 \\
\hline 10 & 364 & 0.01 & 40.33 & 0.04 & -0.01 & 0.00 & 0.00 \\
\hline 530 & 0.25 & 0.01 & 23.65 & 0.03 & 0.11 & 0.00 & 0.00 \\
\hline 530 & 0.5 & 0.00 & 28.60 & 0.02 & -0.03 & 0.00 & 0.00 \\
\hline 530 & 1 & 0.00 & 33.49 & 0.02 & -0.02 & 0.00 & 0.00 \\
\hline 530 & 3 & 0.02 & 48.99 & 0.02 & -0.02 & 0.01 & 0.00 \\
\hline 530 & 7 & 0.02 & 62.03 & 0.04 & -0.01 & 0.01 & 0.00 \\
\hline 530 & 14 & 0.01 & 72.60 & 0.05 & -0.02 & 0.01 & 0.00 \\
\hline 530 & 28 & 0.05 & 93.47 & 0.07 & -0.03 & 0.01 & 0.00 \\
\hline 530 & 56 & 0.03 & 79.53 & 0.06 & 0.03 & 0.01 & 0.01 \\
\hline 530 & 91 & 0.02 & 86.99 & 0.06 & 0.01 & 0.01 & 0.01 \\
\hline 530 & 182 & 0.03 & 94.97 & 0.08 & 0.00 & 0.01 & 0.00 \\
\hline 2000 & 0.25 & -0.03 & 37.69 & 0.01 & -0.05 & 0.00 & 0.00 \\
\hline 2000 & 0.5 & 0.00 & 44.40 & 0.01 & 0.18 & 0.00 & 0.00 \\
\hline 2000 & 1 & 0.01 & 59.13 & 0.01 & 0.04 & 0.00 & 0.00 \\
\hline 2000 & 3 & 0.01 & 83.30 & 0.02 & 0.00 & 0.01 & 0.00 \\
\hline 2000 & 7 & 0.02 & 98.10 & 0.04 & 0.02 & 0.02 & 0.01 \\
\hline 2000 & 14 & 0.03 & 107.33 & 0.05 & 0.00 & 0.02 & 0.01 \\
\hline 2000 & 28 & 0.06 & 115.94 & 0.06 & 0.04 & 0.02 & 0.00 \\
\hline 2000 & 56 & 0.06 & 113.32 & 0.08 & 0.09 & 0.03 & 0.01 \\
\hline 2000 & 91 & 0.07 & 122.33 & 0.11 & 0.01 & 0.03 & 0.02 \\
\hline 2000 & 182 & 0.05 & 138.33 & 0.15 & 0.04 & 0.02 & 0.01 \\
\hline 20000 & 1 & 0.12 & 196.21 & 0.02 & 0.71 & 0.00 & 0.00 \\
\hline 20000 & 3 & 0.01 & 268.54 & 0.04 & 0.25 & 0.06 & 0.00 \\
\hline 20000 & 7 & 0.09 & 323.36 & 0.06 & 0.66 & 0.12 & 0.03 \\
\hline 20000 & 14 & 0.06 & 375.34 & 0.08 & 0.62 & 0.18 & 0.01 \\
\hline 20000 & 28 & 0.09 & 288.27 & 0.01 & 0.21 & 0.00 & 0.01 \\
\hline
\end{tabular}


AVERAGE CONC. (ppm, blank corrected) FOR 0.45u FILTERED LEACHATES

\begin{tabular}{|c|c|c|c|c|}
\hline $\mathrm{SA} / \mathrm{V}$ & Duration & & & \\
\hline$(m-1)$ & (days) & $\mathbf{Y}$ & $\overline{\mathrm{ZN}}$ & $\overline{\mathbf{Z R}}$ \\
\hline 10 & 0.25 & 0.00 & -0.02 & 0.00 \\
\hline 10 & 0.5 & 0.00 & 0.00 & 0.00 \\
\hline 10 & 1 & 0.00 & 0.00 & 0.00 \\
\hline 10 & 3 & 0.00 & 0.00 & 0.00 \\
\hline 10 & 7 & 0.00 & 0.00 & 0.00 \\
\hline 10 & 14 & 0.00 & 0.00 & 0.00 \\
\hline 10 & 28 & 0.00 & 0.00 & -0.01 \\
\hline 10 & 56 & 0.00 & -0.03 & 0.00 \\
\hline 10 & 91 & 0.00 & 0.00 & 0.01 \\
\hline 10 & 182 & 0.00 & 0.00 & 0.00 \\
\hline 10 & 364 & 0,00 & 0.00 & 0.00 \\
\hline 530 & 0.25 & 0.00 & 0.00 & 0.04 \\
\hline 530 & 0.5 & 0.00 & 0.00 & 0.07 \\
\hline 530 & 1 & 0.01 & 0.00 & 0.13 \\
\hline 530 & 3 & 0.01 & 0.00 & 0.11 \\
\hline 530 & 7 & 0.01 & 0.00 & 0.12 \\
\hline 530 & 14 & 0.01 & 0.00 & 0.16 \\
\hline 530 & 28 & 0.02 & 0.00 & 0.33 \\
\hline 530 & 56 & 0.02 & 0.00 & 0.25 \\
\hline 530 & 91 & 0.02 & 0.00 & 0.24 \\
\hline 530 & 182 & 0.02 & 0.01 & 0.25 \\
\hline 2000 & 0.25 & 0.00 & 0.00 & 0.03 \\
\hline 2000 & 0.5 & 0.00 & 0.00 & 0.04 \\
\hline 2000 & 1 & 0.01 & 0.00 & 0.08 \\
\hline 2000 & 3 & 0.01 & 0.01 & $\overline{0.15}$ \\
\hline 2000 & 7 & 0.02 & 0.01 & 0.21 \\
\hline 2000 & 14 & 0.03 & 0.00 & 0.26 \\
\hline 2000 & 28 & 0.04 & 0.01 & 0.32 \\
\hline 2000 & 56 & 0.05 & 0.01 & 0.59 \\
\hline 2000 & 91 & 0.07 & 0.01 & 0.27 \\
\hline 2000 & 182 & 0.11 & 0.01 & 0.18 \\
\hline 20000 & 1 & 0.00 & 0.00 & 0.15 \\
\hline 20000 & 3 & 0.04 & 0.02 & 0.63 \\
\hline 20000 & 7 & 0.08 & 0.05 & 1.05 \\
\hline 20000 & 14 & 0.13 & 0.03 & 1.59 \\
\hline 20000 & 28 & 0.00 & 0.08 & 0.15 \\
\hline
\end{tabular}


. 
AVERAGE NORM. CONC. (g/m2, blank corrected) FOR 18A FILTERED LEACHATES

\begin{tabular}{|c|c|c|c|c|c|c|c|}
\hline $\mathrm{SA} / \mathrm{V}$ & Duration & & & & & & \\
\hline$(m-1)$ & (days) & pH final & $\mathrm{pH}$ in & $\mathrm{AL}$ & AS & $\bar{B}$ & $\overline{B A}$ \\
\hline 10 & 0.25 & 7.36 & 5.58 & 3.57 & 0.00 & 3.52 & 0.00 \\
\hline 10 & 0.5 & 9.02 & 5.88 & 15.17 & 0.00 & 16.62 & 2.20 \\
\hline 10 & 1 & 9.51 & 5.67 & 28.73 & -132033.49 & 31.05 & 8.68 \\
\hline 10 & 3 & 9.67 & 5.37 & 61.05 & -44011.16 & 70.31 & 15.30 \\
\hline 10 & 7 & 9.52 & 5.74 & 73.98 & 123231.25 & 98.99 & 11.16 \\
\hline 10 & 14 & 9.71 & 5.74 & 82.74 & -132033.49 & 121.06 & 9.51 \\
\hline 10 & 28 & 9.32 & 6.01 & 91.09 & 105626.79 & 148.53 & 8.27 \\
\hline 10 & 56 & 9.44 & 6.01 & 94.04 & 202451.34 & 166.29 & 10.75 \\
\hline 10 & 91 & 9.33 & 6.01 & 93.14 & 30807.81 & 181.40 & 11.58 \\
\hline 10 & 182 & 9.44 & 6.01 & 94.92 & -88022.32 & 201.82 & 10.75 \\
\hline 10 & 364 & 8.72 & 6.00 & 84.25 & 74818.98 & 234.20 & 11.99 \\
\hline 530 & 0.25 & 9.90 & 5.58 & 72.53 & 162841.30 & 114.11 & 0.69 \\
\hline 530 & 0.5 & 9.96 & 5.88 & 78.73 & 88022.32 & 145.93 & 0.76 \\
\hline 530 & 1 & 9.95 & 5.74 & 80.15 & -74818.98 & 200.03 & 0.83 \\
\hline 530 & 3 & 9.99 & 5.51 & 93.53 & 92423.44 & 312.37 & 0.41 \\
\hline 530 & 7 & 10.09 & 5.64 & 89.46 & -44011.16 & 420.42 & -10.75 \\
\hline 530 & 14 & 9.99 & 5.69 & 81.02 & -189248.00 & 502.62 & 0.83 \\
\hline 530 & 28 & 10.21 & 5.55 & 77.24 & 48412.28 & 540.06 & -0.51 \\
\hline 530 & 56 & 10.10 & 5.61 & 59.72 & 66016.74 & 590.41 & 0.00 \\
\hline 530 & 91 & 9.88 & 5.61 & 68.72 & -35208.93 & 643.76 & -7.44 \\
\hline 530 & 182 & 9.96 & 5.61 & 50.80 & 26406.70 & 685.83 & 0.41 \\
\hline 2000 & 0.25 & 10.15 & 5.58 & 91.15 & 158440.18 & 214.09 & 0.30 \\
\hline 2000 & 0.5 & 10.18 & 5.88 & 88.54 & 13203.35 & 271.03 & -0.27 \\
\hline 2000 & 1 & 10.04 & 5.69 & 91.64 & -22005.58 & 438.87 & 0.00 \\
\hline 2000 & 3 & 10.31 & 5.51 & 84.47 & -316880.37 & 707.65 & 0.00 \\
\hline 2000 & 7 & 10.38 & 5.74 & 86.26 & 61615.63 & 893.47 & 0.00 \\
\hline 2000 & 14 & 10.28 & 5.69 & 76.95 & 110027.90 & 990.31 & -2.48 \\
\hline 2000 & 28 & 10.55 & 5.55 & 72.13 & 0.00 & 1069.69 & -3.76 \\
\hline 2000 & 56 & 10.42 & 5.61 & 61.79 & 66016.74 & 1165.53 & 0.83 \\
\hline 2000 & 91 & 10.27 & 5.61 & 73.83 & -17604.46 & 1262.86 & 3.72 \\
\hline 2000 & 182 & 10.35 & 5.61 & 62.39 & 184846.88 & 1434.32 & 0.83 \\
\hline 20000 & 1 & 10.78 & 5.58 & 47.78 & 849065.62 & 2188.78 & 0.29 \\
\hline 20000 & 3 & 10.89 & 5.51 & 16.87 & -328763.38 & 3440.38 & 0.00 \\
\hline 20000 & 7 & 10.89 & 5.64 & 24.17 & -612151.25 & 3991.08 & -6.20 \\
\hline 20000 & 14 & 10.93 & 5.64 & 24.81 & 0.00 & 4533.48 & 0.00 \\
\hline 20000 & 28 & 11.20 & 5.55 & 21.98 & 96824.56 & 5141.72 & 0.85 \\
\hline
\end{tabular}


AVERAGE NORM. CONC. (g/m2, blank corrected) FOR 18A FILTERED LEACHATES

\begin{tabular}{|c|c|c|c|c|c|c|c|}
\hline $\mathrm{SA} / \mathrm{V}$ & Duration & & & & & & \\
\hline$(m-1)$ & (days) & $\overline{\mathbf{B E}}$ & $\overline{C A}$ & $\overline{C D}$ & $\mathrm{CE}$ & $\mathrm{CO}$ & $\overline{C R}$ \\
\hline 10 & 0.25 & 0.00 & 1.34 & -3807.83 & 2.14 & 0.00 & 0.00 \\
\hline 10 & 0.5 & 0.00 & 13.09 & -19039.15 & 0.00 & 0.00 & 0.00 \\
\hline 10 & 1 & 0.00 & 22.70 & 7615.66 & 0.00 & 0.00 & 0.08 \\
\hline 10 & 3 & 0.00 & 52.31 & 0.00 & 0.00 & 0.00 & -0.04 \\
\hline 10 & 7 & 0.00 & 62.51 & 0.00 & 0.00 & 0.00 & 0.15 \\
\hline 10 & 14 & 0.00 & 65.70 & 0.00 & 0.00 & 0.00 & 0.19 \\
\hline 10 & 28 & 0.00 & 72.36 & -3807.83 & 0.00 & -14072.41 & 0.31 \\
\hline 10 & 56 & 0.00 & 74.07 & 3807.83 & 0.00 & 4690.80 & 0.69 \\
\hline 10 & 91 & 0.00 & 71.26 & 0.00 & 0.00 & 0.00 & 0.58 \\
\hline 10 & 182 & 0.00 & 86.80 & 0.00 & 0.00 & 4690.80 & 1.23 \\
\hline 10 & 364 & 0.00 & 88.79 & 11423.49 & 6.41 & 0.00 & 1.11 \\
\hline 530 & 0.25 & 0.00 & 20.78 & -19419.93 & 0.00 & 0.00 & 0.00 \\
\hline 530 & 0.5 & 0.00 & 19.34 & 5711.74 & 0.00 & 0.00 & 0.00 \\
\hline 530 & 1 & 0.00 & 10.90 & 0.00 & 0.00 & 0.00 & 0.31 \\
\hline 530 & 3 & 0.00 & 6.34 & 0.00 & 0.00 & -4690.80 & 0.77 \\
\hline 530 & 7 & 0.00 & 7.44 & 0.00 & 0.00 & 0.00 & 1.97 \\
\hline 530 & 14 & 0.00 & 9.68 & 1142348.75 & 0.00 & 0.00 & 2.17 \\
\hline 530 & 28 & 0.00 & 14.32 & 3807.83 & 0.00 & 0.00 & 3.62 \\
\hline 530 & 56 & 0.00 & 9.62 & 7615.66 & 0.00 & 0.00 & 4.45 \\
\hline 530 & 91 & 0.00 & 17.77 & 0.00 & 0.00 & 0.00 & 5.87 \\
\hline 530 & 182 & 0.00 & 24.39 & 3807.83 & 0.00 & 0.00 & 7.14 \\
\hline 2000 & 0.25 & 0.00 & 2.46 & 1142.35 & 0.00 & 0.00 & 0.00 \\
\hline 2000 & 0.5 & 2775.37 & 2.87 & -13708.19 & 0.00 & 0.00 & 0.05 \\
\hline 2000 & 1 & 0.00 & 0.97 & -11423.49 & 0.00 & 0.00 & 0.93 \\
\hline 2000 & 3 & 0.00 & 3.08 & 0.00 & 0.00 & -14072.41 & 1.82 \\
\hline 2000 & 7 & 0.00 & 7.77 & 0.00 & 0.00 & 0.00 & 4.56 \\
\hline 2000 & 14 & 0.00 & 7.79 & 4607473.31 & 0.00 & 4690.80 & 5.07 \\
\hline 2000 & 28 & 0.00 & 11.02 & 0.00 & 0.00 & 0.00 & 6.65 \\
\hline 2000 & 56 & 0.00 & 15.09 & 0.00 & 0.00 & 0.00 & 10.09 \\
\hline 2000 & 91 & 0.00 & 34.96 & 0.00 & 0.00 & -9381.60 & 13.85 \\
\hline 2000 & 182 & 0.00 & 35.17 & 15231.32 & 0.18 & 14072.41 & 19.07 \\
\hline 20000 & 1 & 0.00 & 4.44 & 43625.92 & 0.00 & 0.00 & 3.32 \\
\hline 20000 & 3 & 0.00 & 4.55 & 0.00 & 0.00 & -70080.59 & 20.12 \\
\hline 20000 & 7 & 0.00 & -24.77 & 0.00 & -37.80 & -351810.19 & 36.87 \\
\hline 20000 & 14 & 0.00 & -8.26 & 19381.85 & 0.00 & 0.00 & 50.38 \\
\hline 20000 & 28 & 0.00 & 14.59 & 0.00 & 0.00 & 103197.65 & 51.56 \\
\hline
\end{tabular}


AVERAGE NORM. CONC. (g/m2, blank corrected) FOR 18A FILTERED LEACHATES

\begin{tabular}{|c|c|c|c|c|c|c|c|}
\hline $\mathrm{SA} / \mathrm{V}$ & Duration & & & & & & \\
\hline$(m-1)$ & (days) & $\mathrm{CS}$ & $\mathrm{CU}$ & DY & EU & FE & GD \\
\hline 10 & 0.25 & 0.64 & 3.13 & 7651.28 & 0.00 & 0.00 & 0.00 \\
\hline 10 & 0.5 & 16.49 & 0.79 & 0.00 & 0.00 & 0.00 & 0.00 \\
\hline 10 & 1 & 35.02 & -3.13 & 0.00 & 0.00 & 0.00 & 0.00 \\
\hline 10 & 3 & 104.41 & 1.04 & 0.00 & 0.00 & 0.00 & -76841.55 \\
\hline 10 & 7 & 136.54 & 2.09 & 0.00 & 0.00 & 0.00 & -115262.32 \\
\hline 10 & 14 & 142.97 & 1.74 & 0.00 & 0.00 & 0.00 & -92209.86 \\
\hline 10 & 28 & 162.24 & 2.09 & 0.00 & 0.00 & 0.00 & -115262.32 \\
\hline 10 & 56 & 187.94 & 4.17 & 0.00 & 0.00 & 0.00 & -153683.09 \\
\hline 10 & 91 & 216.86 & 0.70 & 0.00 & 0.00 & 0.00 & -230524.64 \\
\hline 10 & 182 & 229.71 & 0.00 & 0.00 & 0.00 & 0.00 & -69157.39 \\
\hline 10 & 364 & 277.90 & 11.82 & -3825.64 & 0.00 & 0.00 & -188261.79 \\
\hline 530 & 0.25 & 170.81 & 0.34 & 0.00 & 0.00 & 0.00 & 0.00 \\
\hline 530 & 0.5 & 198.30 & 0.76 & 0.00 & 0.00 & 0.00 & 0.00 \\
\hline 530 & 1 & 147.67 & 0.00 & 0.00 & 0.00 & 0.00 & -115262.32 \\
\hline 530 & 3 & 180.49 & -0.35 & -11476.92 & 0.00 & 0.00 & -115262.32 \\
\hline 530 & 7 & 190.58 & 1.39 & 0.00 & 0.00 & 0.00 & -230524.64 \\
\hline 530 & 14 & 170.39 & 4.17 & 0.00 & 0.00 & 0.00 & -149841.02 \\
\hline 530 & 28 & 237.56 & 0.00 & 0.00 & 0.00 & 0.00 & -230524.64 \\
\hline 530 & 56 & 178.31 & 0.00 & 0.00 & 0.00 & 0.00 & -230524.64 \\
\hline 530 & 91 & 207.22 & 1.04 & 0.00 & 0.00 & 0.00 & -153683.09 \\
\hline 530 & 182 & 213.65 & 0.35 & 0.00 & 0.00 & 0.00 & -230524.64 \\
\hline 2000 & 0.25 & 268.97 & 0.40 & 0.00 & 0.00 & 0.00 & 0.00 \\
\hline 2000 & 0.5 & 233.64 & 0.64 & 0.00 & 0.00 & 0.00 & 0.00 \\
\hline 2000 & 1 & 130.00 & 0.35 & 0.00 & 0.00 & 0.00 & -230524.64 \\
\hline 2000 & 3 & 104.76 & 1.04 & 0.00 & 0.00 & 0.00 & -345786.96 \\
\hline 2000 & 7 & 109.81 & 5.22 & 0.00 & 0.00 & 0.00 & -307366.19 \\
\hline 2000 & 14 & 122.43 & 3.13 & 0.00 & 0.00 & 0.00 & -46104.93 \\
\hline 2000 & 28 & 158.33 & 0.20 & 0.00 & 0.00 & 0.00 & -461049.28 \\
\hline 2000 & 56 & 139.75 & 7.30 & 0.00 & 0.00 & 0.00 & -461049.28 \\
\hline 2000 & 91 & 210.43 & 21.91 & 0.00 & 0.00 & 0.00 & -384207.74 \\
\hline 2000 & 182 & 274.69 & 7.30 & 0.00 & 0.00 & 0.00 & -103736.09 \\
\hline 20000 & 1 & 195.63 & 21.61 & 0.00 & 0.00 & 0.00 & 0.00 \\
\hline 20000 & 3 & 140.56 & 11.85 & 0.00 & 0.00 & 0.00 & \#\#\#\#\#\#\# \\
\hline 20000 & 7 & 200.06 & 8.74 & -229538.46 & -57896.81 & -57896.81 & \#\#\#\#\#\#\# \\
\hline 20000 & 14 & 152.48 & 0.00 & 0.00 & 0.00 & 0.00 & \#\#\#\#\#\#\# \\
\hline 20000 & 28 & 142.14 & 0.00 & 42082.05 & 0.00 & 0.00 & I\#\#\#\#\# \\
\hline
\end{tabular}


AVERAGE NORM. CONC. (g/m2, blank corrected) FOR 18A FILTERED LEACHATES

\begin{tabular}{|c|c|c|c|c|c|c|c|}
\hline $\mathrm{SA} / \mathrm{V}$ & Duration & & & & & & \\
\hline$(m-1)$ & (days) & $\mathbf{K}$ & LA & LI & MG & $\mathbf{M N}$ & MO \\
\hline 10 & 0.25 & 28107.17 & 0.21 & 3.64 & 0.79 & 0.93 & 2.68 \\
\hline 10 & 0.5 & 0.00 & 0.00 & 15.12 & 8.43 & 0.99 & 10.77 \\
\hline 10 & 1 & 0.00 & 0.00 & 29.78 & 20.89 & 1.24 & 26.08 \\
\hline 10 & 3 & 0.00 & 0.00 & 69.92 & 40.20 & 2.48 & 67.87 \\
\hline 10 & 7 & 0.00 & 0.00 & 99.39 & 7.75 & 2.17 & 93.06 \\
\hline 10 & 14 & 0.00 & 0.00 & 121.14 & 2.87 & 2.79 & 116.99 \\
\hline 10 & 28 & 0.00 & 0.00 & 150.20 & 2.94 & 2.48 & 139.49 \\
\hline 10 & 56 & 0.00 & 0.00 & 167.07 & 6.89 & 4.34 & 158.96 \\
\hline 10 & 91 & 0.00 & -2.06 & 179.75 & 2.73 & 5.59 & 172.18 \\
\hline 10 & 182 & 782985.53 & 0.48 & 199.55 & 13.28 & 7.76 & 191.65 \\
\hline 10 & 364 & 0.00 & 1.10 & 236.78 & 16.58 & 9.62 & 228.80 \\
\hline 530 & 0.25 & -245737.00 & 0.11 & 126.50 & 20.88 & 0.49 & 101.42 \\
\hline 530 & 0.5 & 368605.49 & 0.39 & 165.96 & 15.66 & 2.90 & 153.71 \\
\hline 530 & 1 & 0.00 & 0.00 & 214.84 & 0.00 & 0.33 & 205.64 \\
\hline 530 & 3 & 0.00 & -0.21 & 322.38 & 1.91 & 0.66 & 331.27 \\
\hline 530 & 7 & 0.00 & 0.00 & 437.25 & 2.30 & 0.99 & 441.29 \\
\hline 530 & 14 & 0.00 & 0.21 & 494.61 & 5.72 & 4.62 & 501.90 \\
\hline 530 & 28 & 0.00 & 0.00 & 546.70 & 0.00 & -0.33 & 545.11 \\
\hline 530 & 56 & 0.00 & 0.00 & 650.15 & 2.08 & 0.62 & 555.47 \\
\hline 530 & 91 & 176673.66 & 0.00 & 723.20 & 1.36 & 1.24 & 606.73 \\
\hline 530 & 182 & 0.00 & 0.00 & 792.67 & 2.58 & 0.62 & 623.34 \\
\hline 2000 & 0.25 & 184302.75 & 0.00 & 276.87 & 4.19 & 0.33 & 227.28 \\
\hline 2000 & 0.5 & 675776.74 & 0.11 & 323.74 & -0.52 & 2.37 & 263.42 \\
\hline 2000 & 1 & 0.00 & 0.00 & 504.69 & 0.72 & 0.33 & 467.50 \\
\hline 2000 & 3 & 0.00 & 0.00 & 761.08 & -0.39 & 0.99 & 743.55 \\
\hline 2000 & 7 & 0.00 & 0.00 & 943.93 & 2.83 & 2.97 & 886.98 \\
\hline 2000 & 14 & 0.00 & 0.21 & 1038.00 & 3.68 & 2.64 & 978.20 \\
\hline 2000 & 28 & 0.00 & 0.00 & 1173.17 & 0.00 & 5.60 & 1032.75 \\
\hline 2000 & 56 & 0.00 & 0.07 & 1369.90 & 8.40 & 4.65 & 1030.57 \\
\hline 2000 & 91 & 0.00 & 3.02 & 1512.17 & 22.25 & 16.76 & 1132.56 \\
\hline 2000 & 182 & 0.00 & 1.10 & 1770.30 & 9.98 & 5.90 & 1318.13 \\
\hline 20000 & 1 & 0.00 & 0.00 & 2520.04 & 28.67 & -4.21 & 2089.35 \\
\hline 20000 & 3 & 0.00 & 0.00 & 3646.99 & -1.97 & 3.05 & 3281.22 \\
\hline 20000 & 7 & -138.17 & -3.84 & 4237.99 & -29.94 & 11.58 & 3747.24 \\
\hline 20000 & 14 & 0.00 & 0.00 & 4817.94 & 0.00 & 0.00 & 3954.22 \\
\hline 20000 & 28 & 0.00 & 1.26 & 5077.83 & 7.87 & 37.58 & 4694.06 \\
\hline
\end{tabular}


AVERAGE NORM. CONC. (g/m2, blank corrected) FOR 18A FILTERED LEACHATES

\begin{tabular}{|c|c|c|c|c|c|c|c|}
\hline $\mathrm{SA} / \mathrm{V}$ & Duration & & & & & & \\
\hline$(m-1)$ & (days) & NA & ND & NI & $\mathbf{P}$ & PB & RU \\
\hline 10 & 0.25 & 3.34 & 0.00 & 0.14 & -183313.12 & 10772.24 & 0.00 \\
\hline 10 & 0.5 & 14.89 & 0.00 & 0.00 & 0.00 & 0.00 & 0.00 \\
\hline 10 & 1 & 27.86 & 0.00 & 0.00 & 22914.14 & 0.00 & 0.00 \\
\hline 10 & 3 & 65.69 & 0.00 & 0.36 & -137484.84 & 0.00 & 0.00 \\
\hline 10 & 7 & 95.14 & 0.00 & 0.43 & -45828.28 & -179537.30 & 0.00 \\
\hline 10 & 14 & 117.27 & 0.00 & 1.08 & 496473.04 & -251352.22 & 8777.41 \\
\hline 10 & 28 & 135.45 & 0.00 & 0.79 & -137484.84 & -323167.14 & -13166.12 \\
\hline 10 & 56 & 152.32 & 0.00 & 1.01 & 290245.78 & -251352.22 & 17554.83 \\
\hline 10 & 91 & 171.19 & 0.00 & 0.72 & 137484.84 & -430889.52 & 0.00 \\
\hline 10 & 182 & 193.45 & 0.26 & 0.07 & 1153345.06 & -71814.92 & -35109.66 \\
\hline 10 & 364 & 212.74 & 1.38 & 3.24 & 1810217.09 & 204672.52 & 0.00 \\
\hline 530 & 0.25 & 119.67 & 0.00 & -0.83 & 216156.72 & -217240.13 & 1.48 \\
\hline 530 & 0.5 & 154.38 & 0.00 & 0.57 & 386485.17 & -43448.03 & 4.44 \\
\hline 530 & 1 & 190.41 & 0.00 & 0.00 & 465920.85 & -430889.52 & 0.00 \\
\hline 530 & 3 & 290.00 & 0.00 & 0.07 & 908927.57 & -434480.27 & 0.00 \\
\hline 530 & 7 & 395.71 & 0.00 & 0.45 & 1260277.72 & -538611.90 & 0.00 \\
\hline 530 & 14 & 451.90 & 0.00 & 2.08 & 1894235.60 & -718149.20 & 245767.62 \\
\hline 530 & 28 & 526.35 & 0.00 & 0.00 & 1558161.54 & -107722.38 & 0.00 \\
\hline 530 & 56 & 565.53 & 0.00 & -0.07 & 1886597.55 & -574519.36 & 0.00 \\
\hline 530 & 91 & 639.97 & 0.00 & 0.50 & 2046996.54 & -538611.90 & 0.00 \\
\hline 530 & 182 & 666.48 & 0.00 & 0.14 & 2069910.68 & 17953.73 & 17554.83 \\
\hline 2000 & 0.25 & 245.00 & 0.00 & -0.83 & 1225142.70 & 217240.13 & 0.74 \\
\hline 2000 & 0.5 & 293.17 & 0.00 & 0.71 & 644651.15 & 0.00 & 1.48 \\
\hline 2000 & 1 & 408.99 & 0.00 & 0.15 & 1474143.03 & -574519.36 & 0.00 \\
\hline 2000 & 3 & 644.63 & 0.00 & -0.37 & 1458866.93 & -933593.96 & 0.00 \\
\hline 2000 & 7 & 787.89 & 0.00 & 1.49 & 2551107.62 & -861779.04 & 0.00 \\
\hline 2000 & 14 & 874.31 & 0.00 & 1.49 & 2933009.96 & -969501.42 & 219435.37 \\
\hline 2000 & 28 & 990.57 & 0.00 & 1.13 & 1886597.55 & -107722.38 & 0.00 \\
\hline 2000 & 56 & 1096.37 & 0.00 & 2.30 & 3735004.88 & -933593.96 & 0.00 \\
\hline 2000 & 91 & 1229.87 & 1.64 & 11.00 & 4200925.73 & $\# \# \# \# \#$ & 0.00 \\
\hline 2000 & 182 & 1360.73 & 0.52 & 3.81 & 4995282.59 & 265715.21 & 65830.61 \\
\hline 20000 & 1 & 1972.30 & $0 . \overline{00}$ & 2.24 & 5250569.79 & 129452.35 & 0.00 \\
\hline 20000 & 3 & 2795.38 & 0.00 & -2.22 & 5033014.54 & \#\#\#\#\#\# & 0.00 \\
\hline 20000 & 7 & 3253.28 & -17.01 & 0.74 & 5704322.48 & $\# \# \# \# \# \#$ & 0.00 \\
\hline 20000 & 14 & 3619.85 & 0.00 & 0.00 & 8843559.70 & \#\#\#\#\#\#\# & 0.00 \\
\hline 20000 & 28 & 4181.19 & 0.00 & 0.00 & 7531114.12 & 0.00 & 6.03 \\
\hline
\end{tabular}


AVERAGE NORM. CONC. (g/m2, blank corrected) FOR 18A FILTERED LEACHATES

\begin{tabular}{|c|c|c|c|c|c|c|c|}
\hline $\mathrm{SA} / \mathrm{V}$ & Duration & & & & & & \\
\hline$(m-1)$ & (days) & SB & SI & SR & TE & TI & V \\
\hline 10 & 0.25 & 0.00 & 3.12 & 1.48 & 41692.79 & 0.00 & 0.00 \\
\hline 10 & 0.5 & 0.00 & 15.19 & 12.32 & 0.00 & 0.00 & 0.00 \\
\hline 10 & 1 & 0.00 & 27.34 & 21.19 & 0.00 & 0.00 & 0.00 \\
\hline 10 & 3 & 59856.26 & 60.22 & 47.80 & 4169.28 & 0.00 & 0.00 \\
\hline 10 & 7 & 115722.11 & 81.07 & 55.68 & -120909.09 & 0.00 & 0.00 \\
\hline 10 & 14 & 0.00 & 97.13 & 56.17 & -166771.16 & 0.00 & 0.00 \\
\hline 10 & 28 & 43894.59 & 112.16 & 57.16 & -125078.37 & 0.00 & 0.00 \\
\hline 10 & 56 & 87789.19 & 123.36 & 58.14 & 50031.35 & 0.00 & 4903.75 \\
\hline 10 & 91 & 3990.42 & 133.56 & 59.62 & -333542.32 & 0.00 & 0.00 \\
\hline 10 & 182 & 3990.42 & 147.75 & 57.65 & -208463.95 & 2.78 & 0.00 \\
\hline 10 & 364 & 107741.27 & 168.20 & 58.14 & -158432.60 & 0.00 & 14711.24 \\
\hline 530 & 0.25 & 0.00 & 96.52 & 15.28 & 531583.07 & 0.00 & 0.00 \\
\hline 530 & 0.5 & -138866.53 & 117.60 & 10.89 & 0.00 & 0.00 & 0.00 \\
\hline 530 & 1 & 31923.34 & 138.05 & 5.63 & -291849.53 & 0.00 & 0.00 \\
\hline 530 & 3 & 195530.46 & 198.46 & 3.38 & -166771.16 & 0.00 & 4903.75 \\
\hline 530 & 7 & 195530.46 & 241.66 & 5.07 & -208463.95 & 0.00 & 4903.75 \\
\hline 530 & 14 & 239425.05 & 277.67 & 5.63 & -250156.74 & 0.00 & 0.00 \\
\hline 530 & 28 & 247405.89 & 289.48 & 7.88 & -458620.69 & 0.00 & 0.00 \\
\hline 530 & 56 & 71827.52 & 289.56 & 3.94 & 87554.86 & 0.00 & 9807.49 \\
\hline 530 & 91 & 339185.49 & 319.76 & 9.36 & 225141.07 & 0.00 & 29422.48 \\
\hline 530 & 182 & 299281.31 & 340.94 & 11.33 & -291849.53 & 0.00 & 14711.24 \\
\hline 2000 & 0.25 & 173583.16 & 156.97 & 0.64 & 1593498.43 & 0.00 & 0.00 \\
\hline 2000 & 0.5 & 34716.63 & 183.15 & 1.18 & 265166.14 & 0.00 & 0.00 \\
\hline 2000 & 1 & 127693.36 & 241.94 & 0.00 & 237648.90 & 0.00 & 0.00 \\
\hline 2000 & 3 & 71827.52 & 342.10 & 1.69 & -125078.37 & 0.00 & 0.00 \\
\hline 2000 & 7 & 239425.05 & 391.72 & 3.38 & 283510.97 & 0.00 & 24518.73 \\
\hline 2000 & 14 & 339185.49 & 427.30 & 3.94 & 266833.86 & 0.00 & 24518.73 \\
\hline 2000 & 28 & 474859.69 & 448.95 & 6.19 & -62539.18 & 7.41 & 0.00 \\
\hline 2000 & 56 & 267357.97 & 451.78 & 5.42 & 354388.71 & 0.00 & 29422.48 \\
\hline 2000 & 91 & 263367.56 & 494.80 & 13.80 & -150094.04 & 2.78 & 53941.21 \\
\hline 2000 & 182 & 714284.74 & 571.31 & 16.26 & -500313.48 & 0.00 & 78459.95 \\
\hline 20000 & 1 & 520207.91 & 782.48 & 0.00 & 4646225.09 & 0.00 & 0.00 \\
\hline 20000 & 3 & 0.00 & 918.07 & 0.00 & 2109488.40 & 0.00 & 0.00 \\
\hline 20000 & 7 & -412250.03 & 1010.64 & -8.45 & 7314708.15 & -27.80 & -220668.60 \\
\hline 20000 & 14 & 406224.50 & 1136.88 & 0.00 & 5481809.72 & 0.00 & 0.00 \\
\hline 20000 & 28 & 1488425.74 & 1220.95 & 4.93 & 2689184.95 & 0.00 & 0.98 \\
\hline
\end{tabular}


AVERAGE NORM. CONC. (g/m2, blank corrected) FOR 18A FILTERED LEACHATES

\begin{tabular}{|c|c|c|c|c|}
\hline $\mathrm{SA} / \mathrm{V}$ & Duration & & & \\
\hline$(m-1)$ & (days) & $\mathbf{Y}$ & $\overline{Z N}$ & ZR \\
\hline 10 & 0.25 & 0.00 & -236504.51 & 0.00 \\
\hline 10 & 0.5 & 0.00 & 4149.20 & 0.00 \\
\hline 10 & 1 & 0.00 & 0.00 & 0.00 \\
\hline 10 & 3 . & 0.00 & 0.00 & 0.00 \\
\hline 10 & 7 & 0.00 & -33193.62 & 0.08 \\
\hline 10 & 14 & 0.00 & -12447.61 & 0.00 \\
\hline 10 & 28 & 0.00 & -8298.40 & -2.57 \\
\hline 10 & 56 & 0.00 & -340234.56 & 0.08 \\
\hline 10 & 91 & 0.00 & 0.00 & 1.77 \\
\hline 10 & 182 & 0.00 & 0.00 & 0.00 \\
\hline 10 & 364 & 0.00 & 4149.20 & 0.00 \\
\hline 530 & 0.25 & 0.00 & 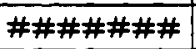 & 0.00 \\
\hline 530 & 0.5 & 0.00 & 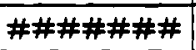 & 0.00 \\
\hline 530 & 1 & 0.00 & $\# \# \# \# \# \#$ & 0.00 \\
\hline 530 & 3 & 0.00 & -269698.13 & 0.93 \\
\hline 530 & 7 & 0.00 & -755154.76 & $\overline{0.17}$ \\
\hline 530 & 14 & 0.00 & 3186587.12 & 1.53 \\
\hline 530 & 28 & 0.00 & 2107794.61 & 0.08 \\
\hline 530 & 56 & 0.00 & 2140988.22 & -0.24 \\
\hline 530 & 91 & 0.00 & 443964.61 & 1.37 \\
\hline 530 & 182 & 0.00 & 3609805.72 & 0.48 \\
\hline 2000 & 0.25 & 0.00 & \#\#\#\#\#\#\# & 0.00 \\
\hline 2000 & 0.5 & 0.00 & \#\#\#\#\#\# & 0.00 \\
\hline 2000 & 1 & 0.00 & 3522672.48 & 0.59 \\
\hline 2000 & 3 & 0.00 & 1029002.09 & $\overline{0.76}$ \\
\hline 2000 & 7 & 0.00 & 2257165.88 & 2.12 \\
\hline 2000 & 14 & 0.00 & 4070367.14 & 1.53 \\
\hline 2000 & 28 & 0.00 & 1933528.12 & 1.89 \\
\hline 2000 & 56 & 0.00 & 6277742.59 & 2.25 \\
\hline 2000 & 91 & 3.17 & 9754773.85 & 7.48 \\
\hline 2000 & 182 & 2.12 & 6742453.22 & $\overline{2.49}$ \\
\hline 20000 & 1 & 0.00 & 3753824.44 & 0.00 \\
\hline 20000 & 3 & 0.00 & \#\#\#\#\#\# & 5.87 \\
\hline 20000 & 7 & -15.87 & \#\#\#\#\#\# & 9.03 \\
\hline 20000 & 14 & 0.00 & 2216669.66 & 6.42 \\
\hline 20000 & 28 & 0.00 & \#\#\#\#\#\#\# & 10.01 \\
\hline
\end{tabular}


$-$ 
AVERAGE NORM. CONC. (g/m2, blank corrected) for 0.45u FILTER LEACHATES

\begin{tabular}{|c|c|c|c|c|c|c|c|c|}
\hline & $\bar{A}$ & $\mathbf{B}$ & $\bar{C}$ & D & $\overline{\mathbf{E}}$ & $\mathbf{F}$ & $\mathbf{G}$ & $\overline{\mathrm{H}}$ \\
\hline 1 & SA/V & Duration & & & & & & \\
\hline 2 & $(m-1)$ & (days) & pH final & $\mathrm{pH}$ in & $\overline{A L}$ & AS & B & $\mathrm{BA}$ \\
\hline 3 & 10 & 0.25 & 7.36 & 5.58 & 3.57 & 0.00 & 3.52 & 0.00 \\
\hline 4 & 10 & 0.5 & 9.02 & 5.88 & 15.17 & 0.00 & 16.62 & 2.20 \\
\hline 5 & 10 & 1 & 9.51 & 5.67 & 28.73 & -132033.49 & 31.05 & 8.68 \\
\hline 6 & 10 & 3 & 9.67 & 5.37 & 61.05 & -44011.16 & 70.31 & 15.30 \\
\hline 7 & 10 & 7 & 9.52 & 5.74 & 73.98 & 123231.25 & 98.99 & 11.16 \\
\hline 8 & 10 & 14 & 9.71 & 5.74 & 82.74 & -132033.49 & 121.06 & 9.51 \\
\hline 9 & 10 & 28 & 9.32 & 6.01 & 91.09 & 105626.79 & 148.53 & 8.27 \\
\hline 10 & 10 & 56 & 9.44 & 6.01 & 94.04 & 202451.34 & 166.29 & 10.75 \\
\hline 11 & 10 & 91 & 9.33 & 6.01 & 93.14 & 30807.81 & 181.40 & 11.58 \\
\hline 12 & 10 & 182 & 9.44 & 6.01 & 94.92 & -88022.32 & 201.82 & 10.75 \\
\hline 13 & 10 & 364 & 8.72 & 6.00 & 84.25 & 74818.98 & 234.20 & 11.99 \\
\hline 14 & 530 & 0.25 & 9.90 & 5.58 & 84.98 & -103866.34 & 113.75 & 7.16 \\
\hline 15 & 530 & 0.5 & 9.96 & 5.88 & 90.60 & 45771.61 & 145.44 & 7.02 \\
\hline 16 & 530 & 1 & 9.95 & 5.74 & 101.53 & -176044.65 & 196.30 & 26.05 \\
\hline 17 & 530 & 3 & 9.99 & 5.51 & 134.55 & 338885.95 & 305.91 & 32.67 \\
\hline 18 & 530 & 7 & 10.09 & 5.64 & 176.73 & 255264.74 & 412.42 & 52.93 \\
\hline 19 & 530 & 14 & 9.99 & 5.69 & 201.74 & 316880.37 & 488.50 & 66.16 \\
\hline 20 & 530 & 28 & 10.21 & 5.55 & 251.93 & 70417.86 & 532.83 & 38.06 \\
\hline 21 & 530 & 56 & 10.10 & 5.61 & 214.95 & 312479.25 & 583.96 & 69.06 \\
\hline 22 & 530 & 91 & 9.88 & 5.61 & 241.68 & 422507.15 & 624.83 & 72.78 \\
\hline 23 & 530 & 182 & 9.96 & 5.61 & 263.52 & 136434.60 & 677.83 & 87.67 \\
\hline 24 & 2000 & 0.25 & 10.15 & 5.58 & 101.62 & 90662.99 & 211.19 & 3.52 \\
\hline 25 & 2000 & 0.5 & 10.18 & 5.88 & 108.06 & 109587.79 & 268.72 & $\overline{3.35}$ \\
\hline 26 & 2000 & 1 & 10.04 & 5.69 & 123.93 & 228858.04 & 435.68 & 12.41 \\
\hline 27 & 2000 & 3 & 10.31 & 5.51 & 165.09 & -52813.39 & 692.84 & 28.95 \\
\hline 28 & 2000 & 7 & 10.38 & 5.74 & 241.75 & 528133.94 & 872.62 & 43.83 \\
\hline 29 & 2000 & 14 & 10.28 & 5.69 & 281.75 & 462117.20 & 975.05 & 55.00 \\
\hline 30 & 2000 & 28 & 10.55 & 5.55 & 333.48 & 312479.25 & 1061.43 & 45.71 \\
\hline 31 & 2000 & 56 & 10.42 & 5.61 & 349.82 & 589749.57 & 1151.44 & 84.77 \\
\hline 32 & 2000 & 91 & 10.27 & 5.61 & 457.52 & 743788.64 & 1252.78 & 117.44 \\
\hline 33 & 2000 & 182 & 10.35 & 5.61 & 537.73 & 448913.85 & 1412.81 & 162.10 \\
\hline 34 & 20000 & 1 & 10.78 & 5.58 & 148.42 & 289197.70 & 2179.84 & 10.59 \\
\hline 35 & 20000 & 3 & 10.89 & 5.51 & 297.55 & -666240.97 & 3398.20 & 35.38 \\
\hline 36 & 20000 & 7 & 10.89 & 5.64 & 510.13 & 1092004.95 & 3939.27 & 58.38 \\
\hline 37 & 20000 & 14 & 10.93 & 5.64 & 699.33 & -220055.81 & 4475.87 & 72.78 \\
\hline 38 & 20000 & 28 & 11.20 & 5.55 & 23.16 & 44011.16 & 5131.27 & -0.85 \\
\hline
\end{tabular}


AVERAGE NORM. CONC. $(\mathrm{g} / \mathrm{m} 2$, blank corrected) for $0.45 \mathrm{u}$ FILTER LEACHATES

\begin{tabular}{|c|c|c|c|c|c|c|c|c|}
\hline & $\overline{\mathbf{A}}$ & B & $I$ & $\mathrm{~J}$ & $\overline{\mathbf{K}}$ & $\mathbf{L}$ & $\mathbf{M}$ & $\mathbf{N}$ \\
\hline 1 & SA/V & Duration & & & & & & \\
\hline 2 & $(m-1)$ & (days) & $\overline{\mathrm{BE}}$ & $\overline{C A}$ & CD & $\overline{\mathrm{CE}}$ & $\mathrm{CO}$ & CR \\
\hline 3 & 10 & 0.25 & 0.00 & 1.34 & -3807.83 & 2.14 & 0.00 & 0.00 \\
\hline 4 & 10 & 0.5 & 0.00 & 13.09 & -19039.15 & 0.00 & 0.00 & 0.00 \\
\hline 5 & 10 & 1 & 0.00 & 22.70 & 7615.66 & 0.00 & 0.00 & 0.08 \\
\hline 6 & 10 & 3 & 0.00 & 52.31 & 0.00 & 0.00 & 0.00 & -0.04 \\
\hline 7 & 10 & 7 & 0.00 & 62.51 & 0.00 & 0.00 & 0.00 & 0.15 \\
\hline 8 & 10 & 14 & 0.00 & 65.70 & 0.00 & 0.00 & 0.00 & 0.19 \\
\hline 9 & 10 & 28 & 0.00 & 72.36 & -3807.83 & 0.00 & -14072.41 & 0.31 \\
\hline 10 & 10 & 56 & 0.00 & 74.07 & 3807.83 & 0.00 & 4690.80 & 0.69 \\
\hline 11 & 10 & 91 & 0.00 & 71.26 & 0.00 & 0.00 & 0.00 & 0.58 \\
\hline 12 & 10 & 182 & 0.00 & 86.80 & 0.00 & 0.00 & 4690.80 & 1.23 \\
\hline 13 & 10 & 364 & 0.00 & 88.79 & 11423.49 & 6.41 & 0.00 & 1.11 \\
\hline 14 & 530 & 0.25 & 0.00 & 46.68 & -19800.71 & 0.00 & 0.00 & 1.49 \\
\hline 15 & 530 & 0.5 & 1850.25 & 44.73 & -1523.13 & 6.63 & 0.00 & 2.80 \\
\hline 16 & 530 & 1 & 0.00 & 47.56 & 0.00 & 8.35 & 14072.41 & 6.96 \\
\hline 17 & 530 & 3 & 0.00 & 44.38 & 0.00 & 22.21 & 75052.84 & 6.15 \\
\hline 18 & 530 & 7 & 0.00 & 70.60 & 0.00 & 20.47 & 46908.02 & 7.54 \\
\hline 19 & 530 & 14 & 0.00 & 96.27 & 7672775.80 & 30.55 & 28144.81 & 8.62 \\
\hline 20 & 530 & 28 & 0.00 & 133.00 & 7615.66 & 52.67 & 9381.60 & 14.77 \\
\hline 21 & 530 & 56 & 0.00 & 109.64 & 19039.15 & 42.90 & 65671.23 & 13.20 \\
\hline 22 & 530 & 91 & 0.00 & 123.79 & 0.00 & 45.40 & 84434.44 & 15.31 \\
\hline 23 & 530 & 182 & 0.00 & 152.41 & 11423.49 & 43.44 & 18763.21 & 20.79 \\
\hline 24 & 2000 & 0.25 & 1850.25 & 14.31 & 19039.15 & 0.00 & 0.00 & 1.34 \\
\hline 25 & 2000 & 0.5 & 3700.49 & 12.88 & -13327.40 & 0.00 & 0.00 & 1.66 \\
\hline 26 & 2000 & 1 & 0.00 & 16.73 & 7615.66 & 14.80 & 89125.25 & 4.52 \\
\hline 27 & 2000 & 3 & 0.00 & 45.05 & 0.00 & 13.39 & 37526.42 & 9.47 \\
\hline 28 & 2000 & 7 & 0.00 & 82.30 & 0.00 & 51.97 & 229849.32 & 17.17 \\
\hline 29 & 2000 & 14 & 0.00 & 101.82 & $\# \# \# \# \# \# \#$ & 64.10 & 187632.10 & 20.03 \\
\hline 30 & 2000 & 28 & 0.00 & 135.23 & 19039.15 & 74.42 & 107888.46 & 23.09 \\
\hline 31 & 2000 & 56 & 0.00 & 150.88 & 34270.46 & 131.38 & 281448.15 & 35.71 \\
\hline 32 & 2000 & 91 & 0.00 & 215.09 & 0.00 & 180.16 & 323665.37 & 48.68 \\
\hline 33 & 2000 & 182 & 0.00 & 294.51 & 34270.46 & 275.05 & 253303.33 & 69.09 \\
\hline 34 & 20000 & 1 & 15353.17 & 43.57 & 89742.31 & 0.00 & 0.00 & 11.22 \\
\hline 35 & 20000 & 3 & 0.00 & 98.08 & 0.00 & 9.04 & 1240811.07 & 45.10 \\
\hline 36 & 20000 & 7 & 0.00 & 148.16 & 0.00 & 152.64 & 1563819.73 & 84.62 \\
\hline 37 & 20000 & 14 & 0.00 & 164.24 & 95538.43 & 178.13 & 1650646.48 & 134.36 \\
\hline 38 & 20000 & 28 & 0.00 & 11.95 & 0.00 & 0.00 & 126651.67 & 56.86 \\
\hline
\end{tabular}


AVERAGE NORM. CONC. (g/m2, blank corrected) for 0.45u FILTER LEACHATES

\begin{tabular}{|c|c|c|c|c|c|c|c|c|}
\hline & $\bar{A}$ & $\overline{\mathbf{B}}$ & $\mathbf{0}$ & $\mathbf{P}$ & $\overline{\mathbf{Q}}$ & $\mathbf{R}$ & $\overline{\mathbf{S}}$ & $\mathbf{T}$ \\
\hline 1 & SA/V & Duration & & & & & & \\
\hline 2 & $(m-1)$ & (days) & $C S$ & $\overline{\mathrm{CU}}$ & $\overline{\mathrm{DY}}$ & $\overline{E U}$ & $\overline{F E}$ & GD \\
\hline 3 & 10 & 0.25 & 0.64 & 3.13 & 7651.28 & 0.00 & 0.00 & 0.00 \\
\hline 4 & 10 & 0.5 & 16.49 & 0.79 & 0.00 & 0.00 & 0.00 & 0.00 \\
\hline 5 & 10 & 1 & 35.02 & -3.13 & 0.00 & 0.00 & 0.00 & 0.00 \\
\hline 6 & 10 & 3 & 104.41 & 1.04 & 0.00 & 0.00 & 0.00 & -76841.55 \\
\hline 7 & 10 & 7 & 136.54 & 2.09 & 0.00 & 0.00 & 0.00 & -115262.32 \\
\hline 8 & 10 & 14 & 142.97 & 1.74 & 0.00 & 0.00 & 0.00 & -92209.86 \\
\hline 9 & 10 & 28 & 162.24 & 2.09 & 0.00 & 0.00 & 0.00 & -115262.32 \\
\hline 10 & 10 & 56 & 187.94 & 4.17 & 0.00 & 0.00 & 0.00 & -153683.09 \\
\hline 11 & 10 & 91 & 216.86 & 0.70 & 0.00 & 0.00 & 0.00 & -230524.64 \\
\hline$\overline{12}$ & 10 & 182 & 229.71 & 0.00 & 0.00 & 0.00 & 0.00 & -69157.39 \\
\hline$\overline{13}$ & 10 & 364 & 277.90 & 11.82 & -3825.64 & 0.00 & 0.00 & -188261.79 \\
\hline 14 & 530 & 0.25 & 194.37 & 20.05 & 0.00 & 0.00 & 0.00 & 0.00 \\
\hline 15 & 530 & 0.5 & 221.86 & 28.63 & 0.00 & 0.00 & 0.00 & 0.00 \\
\hline 16 & 530 & 1 & 160.29 & 77.89 & 0.00 & 0.00 & 0.00 & -230524.64 \\
\hline 17 & 530 & 3 & 228.45 & 95.28 & 0.00 & 3859.79 & 3859.79 & -153683.09 \\
\hline 18 & 530 & 7 & 299.13 & 148.48 & 0.00 & 0.00 & 0.00 & -422628.5 \\
\hline 19 & 530 & 14 & 301.65 & 192.64 & -11476.92 & 11579.36 & 11579.36 & -49947.01 \\
\hline 20 & 530 & 28 & 469.23 & 155.98 & 0.00 & 0.00 & 0.00 & -691573.93 \\
\hline 21 & 530 & 56 & 348.58 & 239.23 & 0.00 & 3859.79 & 3859.79 & -345786.96 \\
\hline 22 & 530 & 91 & 419.26 & 271.92 & 3825.64 & 11579.36 & 11579.36 & -307366.19 \\
\hline 23 & 530 & 182 & 470.66 & 315.73 & 0.00 & 0.00 & 0.00 & -499470.06 \\
\hline 24 & 2000 & 0.25 & 292.53 & 21.70 & 0.00 & 0.00 & 0.00 & 0.00 \\
\hline 25 & 2000 & 0.5 & 265.05 & 23.49 & 0.00 & 0.00 & 0.00 & 0.00 \\
\hline 26 & 2000 & 1 & 155.24 & 73.02 & 0.00 & 0.00 & 0.00 & -230524.64 \\
\hline 27 & 2000 & 3 & 170.39 & 181.16 & -11476.92 & 0.00 & 0.00 & -591679.92 \\
\hline 28 & 2000 & 7 & 251.16 & 412.05 & 0.00 & 0.00 & 0.00 & -384207.74 \\
\hline 29 & 2000 & 14 & 306.70 & 475.34 & 11476.92 & 7719.58 & 7719.58 & -437996.82 \\
\hline 30 & 2000 & 28 & 403.74 & 455.57 & 0.00 & 0.00 & 0.00 & 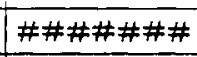 \\
\hline 31 & 2000 & 56 & 422.47 & 699.62 & 7651.28 & 15439.15 & 15439.15 & -653153.15 \\
\hline 32 & 2000 & 91 & 628.08 & 880.44 & 7651.28 & 23158.73 & 23158.73 & -537890.83 \\
\hline 33 & 2000 & 182 & 833.70 & 932.60 & 0.00 & 15439.15 & 15439.15 & -960519.34 \\
\hline 34 & 20000 & 1 & 279.71 & 484.56 & 0.00 & 0.00 & 0.00 & 0.00 \\
\hline 35 & 20000 & 3 & 317.78 & 2359.02 & 0.00 & 0.00 & 0.00 & \#\#\#\#\#\# \\
\hline 36 & 20000 & 7 & 503.14 & 4220.58 & -153025.64 & 19298.94 & 19298.94 & \#\#\#\#\#\# \\
\hline 37 & 20000 & 14 & 412.69 & 5096.46 & 0.00 & 0.00 & 0.00 & $\# \# \# \# \# \#$ \\
\hline 38 & 20000 & 28 & 166.10 & 7.15 & 84164.10 & 0.00 & 0.00 & $\# \# \# \# \#$ \\
\hline
\end{tabular}


AVERAGE NORM. CONC. (g/m2, blank corrected) for 0.45 u FILTER LEACHATES

\begin{tabular}{|c|c|c|c|c|c|c|c|c|}
\hline & $\overline{\mathbf{A}}$ & $\overline{\mathbf{B}}$ & $\overline{\mathbf{U}}$ & $\bar{V}$ & $\overline{\mathbf{W}}$ & $\bar{X}$ & $\bar{Y}$ & $\overline{\mathbf{Z}}$ \\
\hline 1 & SA/V & Duration & & & & & & \\
\hline 2 & $(m-1)$ & (days) & $\mathbf{K}$ & LA & $\mathbf{L I}$ & MG & $\mathrm{MN}$ & $\mathrm{MO}$ \\
\hline 3 & 10 & 0.25 & 28107.17 & 0.21 & 3.64 & 0.79 & 0.93 & 2.68 \\
\hline 4 & 10 & 0.5 & 0.00 & 0.00 & 15.12 & 8.43 & 0.99 & 10.77 \\
\hline 5 & 10 & 1 & 0.00 & 0.00 & 29.78 & 20.89 & 1.24 & 26.08 \\
\hline 6 & 10 & 3 & 0.00 & 0.00 & 69.92 & 40.20 & 2.48 & 67.87 \\
\hline 7 & 10 & 7 & 0.00 & 0.00 & 99.39 & 7.75 & 2.17 & 93.06 \\
\hline 8 & 10 & 14 & 0.00 & 0.00 & 121.14 & 2.87 & 2.79 & 116.99 \\
\hline 9 & 10 & 28 & 0.00 & 0.00 & 150.20 & 2.94 & 2.48 & 139.49 \\
\hline 10 & 10 & 56 & 0.00 & 0.00 & 167.07 & 6.89 & 4.34 & 158.96 \\
\hline 11 & 10 & 91 & 0.00 & -2.06 & 179.75 & 2.73 & 5.59 & 172.18 \\
\hline 12 & 10 & 182 & 782985.53 & 0.48 & 199.55 & 13.28 & 7.76 & 191.65 \\
\hline 13 & 10 & 364 & 0.00 & 1.10 & 236.78 & 16.58 & 9.62 & 228.80 \\
\hline 14 & 530 & 0.25 & 0.00 & 15.03 & 128.75 & 39.18 & 20.14 & 114.14 \\
\hline 15 & 530 & 0.5 & 0.00 & 23.89 & 166.51 & 37.62 & 30.30 & 149.45 \\
\hline 16 & 530 & 1 & 0.00 & 34.28 & 214.24 & 50.47 & 57.69 & 201.84 \\
\hline 17 & 530 & 3 & 0.00 & 29.11 & 322.20 & 125.15 & 93.96 & 327.47 \\
\hline 18 & 530 & 7 & 0.00 & 31.90 & 443.23 & 208.45 & 166.82 & 438.29 \\
\hline 19 & 530 & 14 & -19.84 & 38.05 & 506.20 & 270.69 & 219.57 & 520.51 \\
\hline 20 & 530 & 28 & 0.00 & 58.99 & 566.36 & 350.75 & 288.47 & 562.81 \\
\hline 21 & 530 & 56 & 313194.21 & 44.51 & 670.60 & 285.68 & 209.76 & 544.76 \\
\hline 22 & 530 & 91 & 0.00 & 45.81 & 733.73 & 322.58 & 231.79 & 597.80 \\
\hline 23 & 530 & 182 & 0.00 & 58.36 & 817.06 & 344.54 & 257.54 & 630.49 \\
\hline 24 & 2000 & 0.25 & 245737.00 & 10.60 & 277.84 & 14.63 & 14.47 & 222.10 \\
\hline 25 & 2000 & 0.5 & 122868.50 & 12.92 & 326.61 & 14.11 & 20.47 & 274.61 \\
\hline 26 & 2000 & 1 & 0.00 & 20.45 & 509.06 & 52.24 & 44.18 & 466.10 \\
\hline 27 & 2000 & 3 & 0.00 & 39.09 & 770.68 & 309.71 & 199.13 & 717.55 \\
\hline 28 & 2000 & 7 & 0.00 & 57.73 & 971.99 & 506.51 & 394.63 & 863.58 \\
\hline 29 & 2000 & 14 & 0.00 & 65.55 & 1090.23 & 596.53 & 472.10 & 989.01 \\
\hline 30 & 2000 & 28 & 0.00 & 78.46 & 1257.09 & 712.90 & 560.46 & 1057.91 \\
\hline 31 & 2000 & 56 & 0.00 & 106.03 & 1446.83 & 722.68 & 537.73 & 1019.32 \\
\hline 32 & 2000 & 91 & 0.00 & 146.29 & 1623.96 & 915.19 & 668.05 & 1104.52 \\
\hline 33 & 2000 & 182 & 0.00 & 232.50 & 1898.34 & 913.04 & 686.05 & 1202.04 \\
\hline 34 & 20000 & 1 & 245737.00 & 46.04 & 2581.41 & 830.39 & 478.94 & 2082.98 \\
\hline 35 & 20000 & 3 & 0.00 & 89.19 & 3920.08 & 1835.36 & 1537.57 & 3254.17 \\
\hline 36 & 20000 & 7 & -58.10 & 147.88 & 4734.71 & 2571.49 & 2262.52 & 3719.98 \\
\hline 37 & 20000 & 14 & 0.00 & 228.51 & 5553.77 & 2846.02 & 2664.60 & 4064.61 \\
\hline 38 & 20000 & 28 & 0.00 & 21.85 & 5099.62 & 16.23 & 4.95 & 4723.68 \\
\hline
\end{tabular}


AVERAGE NORM. CONC. (g/m2, blank corrected) for 0.45u FILTER LEACHATES

\begin{tabular}{|c|c|c|c|c|c|c|c|c|}
\hline & $\mathbf{A}$ & B & $\mathbf{A A}$ & $\mathbf{A B}$ & $\mathbf{A C}$ & AD & AE & $\mathbf{A F}$ \\
\hline 1 & SA/V & Duration & & & & & & \\
\hline 2 & $(m-1)$ & (days) & $\overline{\text { NA }}$ & ND & NI & $\mathbf{P}$ & $\overline{\mathrm{PB}}$ & $\overline{R U}$ \\
\hline 3 & 10 & 0.25 & 3.34 & 0.00 & 0.14 & -183313.12 & 10772.24 & 0.00 \\
\hline 4 & 10 & 0.5 & 14.89 & 0.00 & 0.00 & 0.00 & 0.00 & 0.00 \\
\hline 5 & 10 & 1 & 27.86 & 0.00 & 0.00 & 22914.14 & 0.00 & 0.00 \\
\hline 6 & 10 & 3 & 65.69 & 0.00 & 0.36 & -137484.84 & 0.00 & 0.00 \\
\hline 7 & 10 & 7 & 95.14 & 0.00 & 0.43 & -45828.28 & -179537.30 & 0.00 \\
\hline 8 & 10 & 14 & 117.27 & 0.00 & 1.08 & 496473.04 & -251352.22 & 8777.41 \\
\hline 9 & 10 & 28 & 135.45 & 0.00 & 0.79 & -137484.84 & -323167.14 & -13166.12 \\
\hline 10 & 10 & 56 & 152.32 & 0.00 & 1.01 & 290245.78 & -251352.22 & 17554.83 \\
\hline 11 & 10 & 91 & 171.19 & 0.00 & 0.72 & 137484.84 & -430889.52 & 0.00 \\
\hline 12 & 10 & 182 & 193.45 & 0.26 & 0.07 & 1153345.06 & -71814.92 & -35109.66 \\
\hline 13 & 10 & 364 & 212.74 & 1.38 & 3.24 & 1810217.09 & 204672.52 & 0.00 \\
\hline 14 & 530 & 0.25 & 119.93 & 6.81 & 12.49 & 128319.19 & 172355.81 & 1.89 \\
\hline 15 & 530 & 0.5 & 151.75 & 14.52 & 16.27 & 42773.06 & 170201.36 & 4.91 \\
\hline 16 & 530 & 1 & 190.00 & 23.49 & 37.58 & 511749.13 & -430889.52 & 0.00 \\
\hline 17 & 530 & 3 & 289.84 & 25.43 & 52.61 & 992946.08 & -380619.08 & 0.00 \\
\hline 18 & 530 & 7 & 397.98 & 26.73 & 84.32 & 420092.57 & -538611.90 & 0.00 \\
\hline 19 & 530 & 14 & 454.35 & 33.29 & 111.26 & 1726198.57 & -581700.85 & 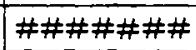 \\
\hline 20 & 530 & 28 & 534.70 & 56.25 & 139.35 & 1168621.16 & -107722.38 & $\overline{46.08}$ \\
\hline 21 & 530 & 56 & 577.52 & 47.26 & 127.19 & 1688008.34 & -682241.74 & $\overline{8777.41}$ \\
\hline 22 & 530 & 91 & 642.09 & 49.16 & 144.44 & 2085186.77 & -646334.28 & 0.00 \\
\hline 23 & 530 & 182 & 681.63 & 57.28 & 167.30 & 1848407.32 & -64633.43 & 746080.27 \\
\hline 24 & 2000 & 0.25 & 243.95 & 3.18 & $\overline{8.14}$ & 602641.89 & 172714.88 & 3.21 \\
\hline 25 & 2000 & 0.5 & 291.94 & 4.99 & 10.39 & -63395.79 & 346506.99 & 1.07 \\
\hline 26 & 2000 & 1 & 413.81 & 18.47 & 34.75 & 1275553.81 & -502704.44 & 57053.20 \\
\hline 27 & 2000 & 3 & 647.03 & 29.00 & 114.38 & 1099878.74 & \#\#\#\#\#\#\# & 0.00 \\
\hline 28 & 2000 & 7 & 795.09 & 54.51 & 238.44 & 1955339.98 & -825871.58 & $0 . \overline{00}$ \\
\hline 29 & 2000 & 14 & 894.96 & 62.69 & 287.04 & 2176843.33 & -897686.50 & \#\#\#\#\#\# \\
\hline 30 & 2000 & 28 & 1030.84 & 76.11 & 308.17 & 832547.10 & -143629.84 & 294091.95 \\
\hline 31 & 2000 & 56 & 1124.16 & 121.05 & 377.03 & 3276722.07 & -908458.74 & -4388.71 \\
\hline 32 & 2000 & 91 & 1289.12 & 170.47 & 488.90 & 3322550.35 & -897686.50 & 0.00 \\
\hline 33 & 2000 & 182 & 1379.43 & 271.29 & 525.57 & 3628072.22 & 68224.17 & 2383068.17 \\
\hline 34 & 20000 & 1 & 1992.50 & 20.43 & 297.00 & 4167052.98 & 748180.26 & 37.26 \\
\hline 35 & 20000 & 3 & 2866.38 & 55.07 & 952.66 & 4976340.24 & \#\#\#\#\#\# & 0.00 \\
\hline 36 & 20000 & 7 & 3372.96 & 152.05 & 1454.91 & 6266253.58 & \#\#\#\#\#\#\# & $0 . \overline{00}$ \\
\hline 37 & 20000 & 14 & 3796.53 & 223.98 & 1669.27 & 6535647.49 & \#\#\#\#\#\#\# & 8450587.95 \\
\hline 38 & 20000 & 28 & 4213.61 & 0.00 & 0.71 & 5774363.37 & 0.00 & 9.05 \\
\hline
\end{tabular}


AVERAGE NORM. CONC. ( $\mathrm{g} / \mathrm{m} 2$, blank corrected) for $0.45 \mathrm{u}$ FILTER LEACHATES

\begin{tabular}{|c|c|c|c|c|c|c|c|c|}
\hline & $\mathbf{A}$ & B & $\mathbf{A G}$ & AH & AI & $\overline{A J}$ & $\mathbf{A K}$ & $\mathbf{A L}$ \\
\hline 1 & SA/V & Duration & & & & & & \\
\hline 2 & $(m-1)$ & (days) & SB & SI & SR & $\mathrm{TE}$ & TI & $\bar{V}$ \\
\hline 3 & 10 & 0.25 & 0.00 & 3.12 & 1.48 & 41692.79 & 0.00 & 0.00 \\
\hline 4 & 10 & 0.5 & 0.00 & 15.19 & 12.32 & 0.00 & 0.00 & 0.00 \\
\hline 5 & 10 & 1 & 0.00 & 27.34 & 21.19 & 0.00 & 0.00 & 0.00 \\
\hline 6 & 10 & 3 & 59856.26 & 60.22 & 47.80 & 4169.28 & 0.00 & 0.00 \\
\hline 7 & 10 & 7 & 115722.11 & 81.07 & 55.68 & -120909.09 & 0.00 & 0.00 \\
\hline 8 & 10 & 14 & 0.00 & 97.13 & 56.17 & -166771.16 & 0.00 & 0.00 \\
\hline 9 & 10 & 28 & 43894.59 & 112.16 & 57.16 & -125078.37 & 0.00 & 0.00 \\
\hline 10 & 10 & 56 & 87789.19 & 123.36 & 58.14 & 50031.35 & 0.00 & 4903.75 \\
\hline 11 & 10 & 91 & 3990.42 & 133.56 & 59.62 & -333542.32 & 0.00 & 0.00 \\
\hline 12 & 10 & 182 & 3990.42 & 147.75 & 57.65 & -208463.95 & 2.78 & 0.00 \\
\hline 13 & 10 & 364 & 107741.27 & 168.20 & 58.14 & -158432.60 & 0.00 & 14711.24 \\
\hline 14 & 530 & 0.25 & 173583.16 & 100.59 & 39.57 & 1328332.29 & 0.00 & 0.00 \\
\hline 15 & 530 & 0.5 & 34716.63 & 121.63 & 34.89 & -397749.22 & 0.56 & 0.00 \\
\hline 16 & 530 & 1 & -39904.18 & 143.60 & 41.11 & -250156.74 & 20.39 & 0.00 \\
\hline 17 & 530 & 3 & 191540.04 & 210.03 & 41.11 & -208463.95 & 27.80 & 49037.47 \\
\hline 18 & 530 & 7 & 223463.38 & 265.95 & 63.07 & -66708.46 & 42.63 & 39229.97 \\
\hline 19 & 530 & 14 & 151635.87 & 311.26 & 82.78 & -250156.74 & 46.33 & 58844.96 \\
\hline 20 & 530 & 28 & 586591.38 & 397.56 & 99.04 & -333542.32 & 56.99 & 1.18 \\
\hline 21 & 530 & 56 & 355147.16 & 331.67 & 84.75 & 412758.62 & 86.18 & 78459.95 \\
\hline 22 & 530 & 91 & 247405.89 & 362.77 & 94.61 & 145924.76 & 97.30 & 93171.19 \\
\hline 23 & 530 & 182 & 307262.15 & 396.04 & 111.85 & -54200.63 & 91.74 & 19614.99 \\
\hline 24 & 2000 & 0.25 & -347166.32 & 160.32 & 10.40 & -663749.22 & 0.00 & 0.00 \\
\hline 25 & 2000 & 0.5 & 34716.63 & 188.85 & 10.15 & 2256830.72 & 0.00 & 0.00 \\
\hline 26 & 2000 & 1 & 115722.11 & 253.50 & 17.46 & 508652.04 & 14.83 & 24518.73 \\
\hline 27 & 2000 & 3 & 107741.27 & 357.11 & 39.42 & 41692.79 & 33.36 & 0.00 \\
\hline 28 & 2000 & 7 & 271348.39 & 420.57 & 64.20 & 300188.09 & 103.79 & 107882.43 \\
\hline 29 & 2000 & 14 & 351156.74 & 460.16 & $\overline{86.72}$ & 25015.67 & 101.94 & 112786.18 \\
\hline 30 & 2000 & 28 & 694332.65 & 494.39 & 97.64 & 516990.60 & 92.21 & 9808.87 \\
\hline 31 & 2000 & 56 & 698323.07 & 472.57 & 112.84 & 1159059.56 & 236.31 & 171631.14 \\
\hline 32 & 2000 & 91 & 782121.83 & 510.16 & 161.62 & 108401.25 & 241.87 & 220668.60 \\
\hline 33 & 2000 & 182 & 570629.71 & 576.89 & 221.25 & 441943.57 & 186.27 & 117689.92 \\
\hline 34 & 20000 & 1 & 1492211.13 & 834.51 & 28.27 & 8838730.38 & 17.80 & 0.00 \\
\hline 35 & 20000 & 3 & 120430.80 & 1151.31 & 66.64 & 3185579.31 & 317.15 & 49037.47 \\
\hline 36 & 20000 & 7 & 1070429.50 & 1386.32 & 99.36 & 8224111.29 & 654.47 & 396762.15 \\
\hline 37 & 20000 & 14 & 741459.48 & 1609.18 & 127.42 & 7781292.16 & 987.97 & 123035.01 \\
\hline 38 & 20000 & 28 & 1077412.73 & 1226.06 & 7.88 & 2634984.33 & 15.29 & 5.10 \\
\hline
\end{tabular}


AVERAGE NORM. CONC. (g/m2, blank corrected) for 0.45u FILTER LEACHATES

\begin{tabular}{|c|c|c|c|c|c|}
\hline & $\mathbf{A}$ & B & $\overline{\mathbf{A M}}$ & $\overline{\mathrm{AN}}$ & $\mathrm{AO}$ \\
\hline 1 & SA/V & Duration & & & \\
\hline 2 & $(m-1)$ & (days) & $\bar{Y}$ & $\overline{\mathrm{ZN}}$ & ZR \\
\hline$\overline{3}$ & $\overline{10}$ & 0.25 & 0.00 & -236504.51 & 0.00 \\
\hline 4 & 10 & 0.5 & 0.00 & 4149.20 & 0.00 \\
\hline 5 & 10 & 1 & 0.00 & 0.00 & 0.00 \\
\hline 6 & 10 & 3 & 0.00 & 0.00 & 0.00 \\
\hline 7 & 10 & 7 & 0.00 & -33193.62 & 0.08 \\
\hline 8 & 10 & 14 & 0.00 & -12447.61 & 0.00 \\
\hline 9 & 10 & 28 & 0.00 & -8298.40 & -2.57 \\
\hline 10 & 10 & 56 & 0.00 & -340234.56 & 0.08 \\
\hline 11 & 10 & 91 & 0.00 & 0.00 & 1.77 \\
\hline 12 & 10 & 182 & 0.00 & 0.00 & 0.00 \\
\hline 13 & 10 & 364 & 0.00 & 4149.20 & 0.00 \\
\hline 14 & 530 & 0.25 & 0.00 & 0.00 & 10.12 \\
\hline 15 & 530 & 0.5 & 0.00 & 2489.52 & 16.76 \\
\hline 16 & 530 & 1 & 30.69 & 12447.61 & 33.81 \\
\hline 17 & 530 & 3 & 30.69 & 41492.02 & 29.22 \\
\hline 18 & 530 & 7 & 33.87 & 16596.81 & 31.09 \\
\hline 19 & 530 & 14 & 44.45 & 33193.62 & 39.76 \\
\hline 20 & 530 & 28 & 10.67 & 41492,02 & 77.87 \\
\hline 21 & 530 & 56 & 56.09 & 37342.82 & 59.82 \\
\hline 22 & 530 & 91 & 61.38 & 24895.21 & 56.69 \\
\hline 23 & 530 & 182 & 77.26 & 78834.84 & 61.03 \\
\hline 24 & 2000 & 0.25 & 0.00 & 0.00 & 6.89 \\
\hline 25 & 2000 & 0.5 & 0.00 & 0.00 & 9.01 \\
\hline 26 & 2000 & 1 & 20.11 & 24895.21 & 19.71 \\
\hline 27 & 2000 & 3 & 38.10 & 62238.03 & 38.40 \\
\hline 28 & 2000 & 7 & 68.79 & 87133.24 & 53.52 \\
\hline 29 & 2000 & 14 & 80.43 & 41492.02 & 65.33 \\
\hline 30 & 2000 & 28 & 39.62 & 70536.43 & 76.41 \\
\hline 31 & 2000 & 56 & 146.04 & 82984.04 & 143.04 \\
\hline 32 & 2000 & 91 & 212.72 & 145222.07 & 64.73 \\
\hline 33 & 2000 & 182 & 340.77 & 141072.87 & 43.82 \\
\hline 34 & 20000 & 1 & 0.00 & 18256.49 & 36.10 \\
\hline 35 & 20000 & 3 & 126.02 & 284054.37 & 160.62 \\
\hline 36 & 20000 & 7 & 245.61 & 648520.27 & 268.45 \\
\hline 37 & 20000 & 14 & 404.44 & 336790.72 & 404.64 \\
\hline 38 & 20000 & 28 & 0.85 & 987510.07 & 34.55 \\
\hline
\end{tabular}


. . . 


\section{APPENDIX B}

QUANTIFYING THE UNCERTAINTY IN NORMALIZED CONCENTRATIONS 
.

, 


\section{APPENDIX B}

\section{QUANTIFYING THE UNCERTAINTY IN NORMALIZED CONCENTRATIONS}

In order to make defensible statements about the chemical durability of the glass tested, it is necessary to quantify the uncertainty in the calculated normalized concentration values. Section B.1 makes some cautionary comments about the sources of variation present in the results considered by this report. Section B. 2 presents the notation and formulas needed to calculate the uncertainty in normalized concentration values. A test case illustrating the use of the formulas is given in Section B.3.

\section{B.1 SOURCES OF VARIATION--CAUTIONARY COMMENTS}

As noted in Section 4.4, normalized concentrations are subject to variation in the glass analysis, leach testing (including sample preparation), and leachate analysis processes. For each of these processes, short-term within-1ab, long-term within-1ab, and 1ab-to-1ab variation occurs. However, it may not be possible to quantify these sources of variation for each of the three processes unless appropriate data are available. For example, to estimate 1ab-to-1ab standard deviations for the glass analysis, leach testing, and leachate analysis processes, data from several laboratories would be needed. Similarly, to estimate long-term within-lab standard deviations, one or more laboratories would have to repeat the glass analysis, leach testing, and leachate analysis processes over an appropriate period of time.

The data discussed in this report were obtained from a single laboratory with replicate glass analyses, leach tests, and leachate analyses performed at the same time. Hence, the data contain only information about short-term within-lab variation. Experience has shown that short-term within-1ab variation is typically a minor contributor to the overall uncertainty in normalized concentration values. This must be kept in mind when considering the standard deviations and other uncertainty information presented in this report. 


\section{B.2 NOTATION AND FORMULAS FOR CALCULATING THE UNCERTAINTY}

IN NORMALIZED CONCENTRATION VALUES

The following notation is introduced for use in the formulas to follow:

$N C_{i j k}=$ normal ized concentration for the $i^{\text {th }}$ element from the $j^{\text {th }}$ specimen (replicate) of the $k^{\text {th }}$ test (nominal SA/V and duration of the test $t$ ) $\begin{aligned} & C_{i j k}= \text { concentration }[\mathrm{ppm}] \text { of element } i \text { reported by ICP (by AA for } C s \text { ) } \\ & \text { in leachate for specimen } j \text { from test } k\end{aligned}$

$B_{i 1}=\begin{aligned} & \text { concentration of element } i \text { for blank test at }(S A / V) \cdot \text { time } \\ & \text { combination } 1\end{aligned}$ $f_{i j m}=$ the mean weight fraction of element $i$ in $g$ lass batch $m$ from the ICP determinations using "K/Ni" and $\mathrm{Na} / \mathrm{Zr}$ " fusions (a single $\mathrm{AA}$ analys is for Cs).

The following notation for the numbers of replicate glass analyses, blank analyses, and leach tests (and leachate analyses) will be helpful.

$\begin{array}{cccc}\text { Variable } & \text { Notation } & \frac{M C C-1}{} & \frac{M C C-3}{3} \\ C_{i j k} & n_{i k} & 3 & 3 \\ B_{i 1} & n_{i 1} & 1 & 2 \text { or } 3 \\ f_{i j m} & n_{i m} & 3 & 3\end{array}$

Note that for both the MCC-1 and MCC-3 tests, three short-term replicate tests were performed for each $S A / V$ and duration combination (i.e., $n_{i k}=3$ ), and the resulting leachates were analyzed once each within a short period of time.

For the MCC-3 tests, there was one blank performed for each of the three $S A / V$ values $\left(530,2,000\right.$, and $\left.20,000 \mathrm{~m}^{-1}\right)$ for each test duration up to 56 days. MCC -3 tests for durations of 91,182 , and 360 days were only performed at $S A / V$ values of 530 and $2000 \mathrm{~m}^{-1}$, so only two values are available. Since the concept of $S A / V$ ratio does not apply to blanks, the two or three blank results available for each MCC-3 test duration can be considered replicates (i.e., $n_{i 1}=2$ or 3 ). There was only one blank performed for each test duration for the MCC-1 tests. 
The $m$ in $f_{i j m}$ and $n_{i m}$ refers to one of the three batched of HW-39-1 used during testing. Each $f_{i j m}$ is usually an average of two ICP determinations, one using the "K/N $\mathrm{N}_{i}$ " fusion calibration standard and the other using the $\mathrm{Na} / \mathrm{Zr}$ " fusion standard. In the case of Cs, only one AA determination is made and used to obtain $f_{i j m}$.

With these definitions and clarifications in mind, the following calculations are defined.

$$
\begin{aligned}
& \overline{c_{i . k}}=\sum_{j=1}^{n} c_{i j k} / n_{i k} \\
& \overline{B_{i .}}=\sum_{i=1}^{n} B_{i 1} B_{i 1} / n_{i 1} \\
& \overline{f_{i . m}}=\sum_{j=1}^{n} f_{i j m} / n_{i m} \\
& N C_{i j k}=\frac{C_{i j k}-\overline{B_{i}}}{f_{i 1 m}} \\
& \overline{N C_{i . k}}=\frac{\overline{C_{i . k}}-\overline{B_{i}}}{f_{i 1 m}}
\end{aligned}
$$

Equations (B-4) and (B-5) are the formulas used to compute, respectively, the individual and mean normalized concentrations in this report. Note that $f_{i 1 m}$, the elemental weight fraction from the first analysis of each batch, was used to compute the normalized concentrations. The normalized concentrations $N C_{i j k}$ and $\overline{N C}_{i . k}$ would be less uncertain if $\bar{f}_{i . m}$ would have been used in $(B-4)$ and $(B-5)$ in place of $f_{i l m}$, but the second and third $g$ hrss analysis were not completed until late in the study.

Next, the variances of interest are given in the following formulas. The "hats" $\left({ }^{\wedge}\right)$ denote estimates of the variances based on the data collected. 
Since the numbers of replicates are small, the estimated variances themselves will be subject to considerable uncertainty.

$$
\begin{aligned}
& \hat{v}\left(C_{i j k}\right)=\frac{1}{n_{i k}-1} \sum_{j=1}^{n_{i k}}\left(C_{i j k}-\bar{C}_{i . k}\right)^{2} \\
& \hat{v}\left(B_{i 1}\right)=\frac{1}{n_{i 1^{-1}}} \sum_{i=1}^{n_{i 1}}\left(B_{i 1}-\bar{B}_{i .}\right)^{2} \\
& \hat{v}\left(f_{i j m}\right)=\frac{1}{n_{i m^{-1}}} \sum_{j=1}^{n i m}\left(f_{i j m}-\bar{f}_{i . m}\right)^{2}
\end{aligned}
$$

Variances of energies of these quantities are also of interest.

$$
\begin{aligned}
& \hat{v}\left(\bar{c}_{i, k}\right)=\frac{1}{n_{i k}} \hat{v}\left(c_{i j k}\right) \\
& \hat{v}\left(\bar{B}_{i .}\right)=\frac{1}{n_{i 1}} \hat{v}\left(B_{i 1}\right) \\
& \hat{v}\left(\bar{f}_{i . m}\right)=\frac{1}{n_{i m}} \hat{v}\left(f_{i j m}\right)
\end{aligned}
$$

Finally, the following formulas give the expressions for the estimated variances of an individual normalized concentration and a mean normalized concentration (averaged over replicate results for a given set of test conditions).

$$
\hat{V}\left(N C_{i j k}\right)=\left(\overline{N C}_{i . k}\right)^{2}\left[\frac{\hat{V}\left(\bar{C}_{i j k}\right)+\hat{V}\left(\bar{B}_{i .}\right)}{\left(\bar{C}_{i . k}-\bar{B}_{i .}\right)^{2}}+\frac{\hat{V}\left(f_{i j m}\right)}{f_{i 1 m}{ }^{2}}\right]
$$




$$
\hat{v}\left(\overline{N C}_{i, k}\right)=\left(\overline{N C}_{i, k}\right)^{2}\left[\frac{\hat{v}\left(\bar{C}_{i, k}\right)+\hat{v}\left(\bar{B}_{i .}\right)}{\left(\bar{C}_{i, k}-\bar{B}_{i .}\right)^{2}}+\frac{\hat{v}\left(f_{i j m}\right)}{f_{i 1 m}{ }^{2}}\right]
$$

The formulas (B-12) and (B-13) are identical except for $\hat{V}\left(C_{i j k}\right)$ in (B.12) being replaced by $\hat{v}\left(\bar{C}_{i, k}\right)$ in $(B-13)$.

Equation (B-12) was used as the basis for computing the error bars on Figures 3.5 and 3.6 . The error bars are $95 \% / 95 \%$ tolerance intervals for individual observations at each set of test conditions. The error bars and the concept of a tolerance interval are discussed in more detail in Section 4.4 .

The variance formulas given in this section are general, but the sources of variation included in the variance estimates depend on the sources of variation included in the data to which the formulas are applied. As noted previous1y, on $1 y$ short-term within-1ab sources of variation are estimable from the data in this study.

\section{B.3 EXAMPLE CALCULATIONS OF NORMALIZED CONCENTRATIONS AND THEIR UNCERTAINTIES}

Example calculations for normalized concentrations and the corresponding estimated variances (and standard deviations) are presented. An example involving the $\mathrm{Al}_{2} \mathrm{O}_{3}$ component of the $\mathrm{HW}-39-1 \mathrm{gl}$ ass and $\mathrm{Al}$ concentrations from the MCC -3 tests at $S A / V=530 \mathrm{~m}^{-1}$ is used to illustrate the calculations.

The data for the example are given in the top portion of Table B.1, while the basic calculations are given in the lower portion of the table. Table B.2 illustrates the variance and standard deviation calculations for the example. The first column of Table B.2 111 ustrates the computation of the variance and standard deviation of individual normalized concentration 
IABLE B.1. Basic Calculations for the Al Error Propagation Example(a)

\begin{tabular}{|c|c|c|c|}
\hline$c_{i j k}$ & $B_{i 1}$ & $f_{i, j m}(b)$ & $N C_{i j k}$ \\
\hline $\begin{array}{ll}j=1 & 5.81 \\
j=2 & 6.02 \\
j=3 & 5.91\end{array}$ & $\begin{array}{ll}l=1 & 0.0 \\
l=2 & 0.0 \\
l=3 & \text { No test }\end{array}$ & $\begin{array}{ll}j=1 & 0.022440 \\
j=2 & 0.022228 \\
j=3 & 0.023392\end{array}$ & $\begin{array}{l}256.10 \\
265.35 \\
260.50\end{array}$ \\
\hline
\end{tabular}

Quantity Applicable

Estimated Equations

$\begin{array}{llllll}\text { Average } & \begin{array}{l}\mathrm{B}-1, \mathrm{~B}-2, \mathrm{~B}-3, \\ \mathrm{~B}-5\end{array} & 5.9133 & 0.0 & 0.02269 & 260.65\end{array}$

Variance B-6, B-7, B-8 $0.01103 \quad 0.0 \quad 3.8467 \times 10^{-1} \quad-$ -

$\begin{array}{lllll}\text { Standard Square roots } & 0.10504 & 0.0 & 6.2021 \times 10^{-4} & \ldots\end{array}$

Deviation of variances

Variance B-9, B-10, $\quad 3.6778 \times 10^{-8} \quad 0.0 \quad 1.2822 \times 10^{-7}$

of Mean B-11

Standard Square roots

Deviation of variances

0.06065

0.0

$3.5808 \times 10^{-4}$

of Mean of mean

(a) Test case is for element $i=A 1, S A / V=530,182$ days, Batch $=8$, rows $1-3$ in RSI file A3RG of 30 Jun 1987.

(b) The $f_{i j m}$ are the averages of two determinations of weight fraction oxide $\mathrm{Al}_{2} \mathrm{O}_{3}$. These weight fraction oxide values $(0.0424,0.0420,0.0442)$ are muTtiplied by 0.529241 to give weight fraction element values.

values $\mathrm{NC}_{i j k}$. The second and third columns illustrate the computation of the variance and standard deviation of mean normalized concentration values $\overline{N C}_{i . k}$. The second column uses a value of zero for the variance of the blank correction, whereas the third column uses a value determined as described below.

Note in Table B. 1 that the concentrations of $A 1$ in the blanks were zero, indicating that the $A 1$ concentrations were below detection limits. Because of the zero values, it is not possible to estimate the uncertainty in the values. An estimate of the variance due to blanks can be obtained from the definition of detection limits used by the PNL ICP 1ab. The detection 
TABLE B.2. Variance and Standard Deviation Calculations for Al Example

\begin{tabular}{|c|c|c|c|}
\hline $\begin{array}{l}\text { Component of } \\
\text { Calculation }\end{array}$ & $\begin{array}{l}\text { Uncertainty in an } \\
\text { Individual } N C{ }_{j} j \mathrm{j} \\
\text { [Equation }(B-12)^{\text {] }}\end{array}$ & $\begin{array}{l}\text { Uncertainty in an } \\
\text { Average } \mathrm{NC}_{i}{ }^{k} \\
\text { [Equation }(B-13) \text { ] }\end{array}$ & $\begin{array}{l}\text { Average } \overline{N C}_{i \cdot k} \text { Using } \\
\text { a Non-Zero } \hat{V}\left(\bar{B}_{i}\right) \\
\text { [Equation }(B-13)]\end{array}$ \\
\hline $\bar{c}_{i, k}$ & 5.9133 & 5.9133 & 5.9133 \\
\hline $\bar{B}_{\boldsymbol{i}}$. & 0.00 & 0.00 & 0.00 \\
\hline $\bar{f}_{i, m}$ & 0.02269 & 0.02269 & 0.02269 \\
\hline$\overline{N C}_{i, k}$ & 260.6500 & 260.6500 & 260.6500 \\
\hline$\hat{V}(C)$ & 0.011033 & $3.6778 \times 10^{-3}$ & $3.6778 \times 10^{-3}$ \\
\hline$\hat{v}\left(\bar{B}_{i}\right)$ & 0.0 & 0.0 & $1.125 \times 10^{-4}$ \\
\hline$\hat{V}(f)$ & $3.8466 \times 10^{-7}$ & $3.8466 \times 10^{-7}$ & $3.8466 \times 10^{-7}$ \\
\hline$(N C)^{2}$ & $21.436 \quad(29.68 \%)$ & $7.1454 \quad(12.34 \%)$ & $7.1454 \quad(12.29 \%)$ \\
\hline$(N B)^{2}$ & $(0 \%)$ & $(0 \%)$ & $0.2186 \quad(0.38 \%)$ \\
\hline$(N f)^{2}$ & $50.7738(70.31 \%)$ & $50.7738 \quad(87.66 \%)$ & $50.7738 \quad(87.33 \%)$ \\
\hline$\hat{v}(N C)$ & $72.210(100.00 \%)$ & $57.9192(100.00 \%)$ & $58.1378(100.00 \%)$ \\
\hline $\operatorname{sd}(N C)$ & 8.4976 & 7.6105 & 7.6248 \\
\hline \%RSD & 3.26 & 2.92 & 2.93 \\
\hline
\end{tabular}

Note: For purposes of assessing the percentage uncertainty contributions of the $C, B$, and $f$ terms of Equation ( $B-12)$, the following expansion is used.

$$
\begin{aligned}
\hat{v}\left(N C_{i j k}\right) & =\frac{\left(\overline{N C}_{i, k}\right)^{2} \hat{v}\left(C_{i j k}\right)}{\left(\bar{C}_{i, k}-\bar{B}_{i .}\right)^{2}}+\frac{\left(\overline{N C}_{i, k}\right)^{2} \hat{v}\left(\bar{B}_{i .}\right)}{\left(\bar{C}_{i, k}-\bar{B}_{i .}\right)^{2}}+\frac{\left(\overline{N C}_{i, k}\right)^{2} \hat{v}\left(f_{i j m}\right)}{\bar{f}_{i l m}{ }^{2}} \\
& =(N C)^{2}+(N B)^{2}+(N f)^{2}
\end{aligned}
$$

A similar expansion of Equation (B-13) is used. 
limit (DL) is defined as the concentration for which the relative standard deviation based on ten determinations is $50 \%$. For the example, the DL for A1 is $0.03 \mu \mathrm{g} / \mathrm{ml}$, so that

$$
\frac{\mathrm{Sd}(\mathrm{AL})}{\mathrm{DL}(\mathrm{A} 1)}=0.5
$$

or

$$
\operatorname{sd}(A 1)=0.5(0.03)=0.015 \mu \mathrm{g} / \mathrm{ml}
$$

MCC -3 test blanks at 182 days were run at SA/V values of 530 and 2000 SA/V so that $n_{i 1}$ is two. An estimate of the variance of the mean to be associated with the blank correction (of zero), is

$$
\hat{V}\left(\bar{B}_{i .}\right)=\frac{(0.015)^{2}}{2}=1.125 \times 10^{-4} \text {. }
$$

This quantity is used in the last column of Table B.2.

It is interesting to note in Table B.2 that the greatest portion of the short-term uncertainty in the normalized $A 1$ concentrations is from the analysis of the glass. Leach testing and analysis of the leachate contributes a smaller but significant uncertainty, while the blank analysis contributes almost no uncertainty. The percentage allocation shown in Table B.2 for A1 can be expected to vary for different elements and different test conditions.

As noted earlier, a single glass analys is was used to calculate the normalized concentrations in this report, although triplicate results for each batch of glass were used to estimate the uncertainty in a single analysis. Using the average of the triplicate analyses to calculate the normalized concentrations would reduce their uncertainty considerably.

To see the benefits for the example calculations of Table B.2, use $\hat{v}(f)=\left(3.8466 \times 10^{-7}\right) / 3=1.2822 \times 10^{-7}$ in place of $3.8466 \times 10^{-7}$ in the calculations. The resulting values of $s d(N C)$ in the three columns are 6.19 , 4.91 , and 4.93 , respectively. 


\section{DISTRIBUTION}

No. of

Copies

\section{OFFSITE}

10 DOE/Office of Scientific and Technical Information

2 DOE Office of Defense Waste \& GTN Transportation Management

Washington, DC 20545

ATTN: K. A. Chacey, DP -123

T. B. Hindman, DP-12

J. M. Pope

Process Technology and Engineering West Valley Nuclear Services Co., Inc.

West Valley, New York 14171-0191

W. F. Hot comb

Environmental Protection Agency

Office of Radiation Programs (ANR-460)

401 M Street S.W.

Washington, DC 20460

E. Maestas

DOE West Valley Project

P.0. Box 191

West Valley, NY 14171

3 DOE Idaho Operations Office

550 Second Street

Idaho Fal1s, ID 83401

ATTN: C. R. Enos

M. W. Shupe

J. E. Solecki

M. R. Jugan

DOE Oak Ridge Operations Office

P.0. Box E

Oak Ridge, TN 37830

W. T. Goldston

DOE Savannah River Operations Office

P.0. Box A

Aiken, SC 29801
No. of

Copies

J. K. Bates

Argonne National Laboratory 9700 South Cass Avenue

Argonne, IL 60439

H. Shaw

Lawrence Livermore National Laboratory

University of California

P.0. Box 808

Livermore, CA 94550

4 Oak Ridge National Laboratory

P.0. Box 2008

Oak Ridge, TN 37831

ATTN: W. D. Burch

R. T. Jubin

L. J. Mezga

D. W. Turner

2 Sandia Laboratories

P.0. Box 5800

Albuquerque, NM 87185

ATTN: R. W. Lynch

Technical Library

J. R. Berreth

West inghouse Idaho Nuclear

Co., Inc.

P.0. Box 4000

Idaho Falls, ID 83401

4 E. I. du Pont de Nemours Company

Savannah River Laboratory

Aiken, SC 29801

ATTN: J. R. Knight $773 \mathrm{~A}$

M. J. Plodinec $773 \mathrm{~A}$

C. M. Jantzen $773 \mathrm{~A}$

R. G. Baxter

E. I. du Pont de Nemours Company

Savannah River Plant

B1dg. 704-S

Aiken, SC 29808 
P. A. Saxman

DOE Albuquerque Operations Office

P.0. Box 5400

Albuquerque, NM 87185

2 West Valley Nuclear Services Company

P.0. Box 191

West Valley, NY 14171

ATTN: J. M. Pope

L. Cadoff

\section{ONSITE}

3 DOE Richland Operations Office

R. W. Brown

P. E. Lamont

W. S. Ketola

15 Westinghouse Hanford Company

B. N. Anderson

P. Fel ise

J. M. Henderson

o. L. Kruger

W. C. Miller

K. R. Shah
No. of

Copies

R. A. Smith

M. D. Totten (5)

J. A. Voogd

E. T. Weber

B. A. Wolfe

35 Pacific Northwest Laboratory

C. R. Allen

S. 0. Bates (10)

R. E. Einziger

M. R. Elmore

W. L. Kuhn

J. W. Johnston (3)

D. E. Larson

G. B. Mellinger

J. M. Perez, Jr.

M. E. Peterson

G. F. Piepel (3)

W. A. Ross

G. J. Sevigny

D. H. Siemens

H. D. Smith

J. H. Westsik, Jr.

Publishing Coordination

Technical Report Files (5) 


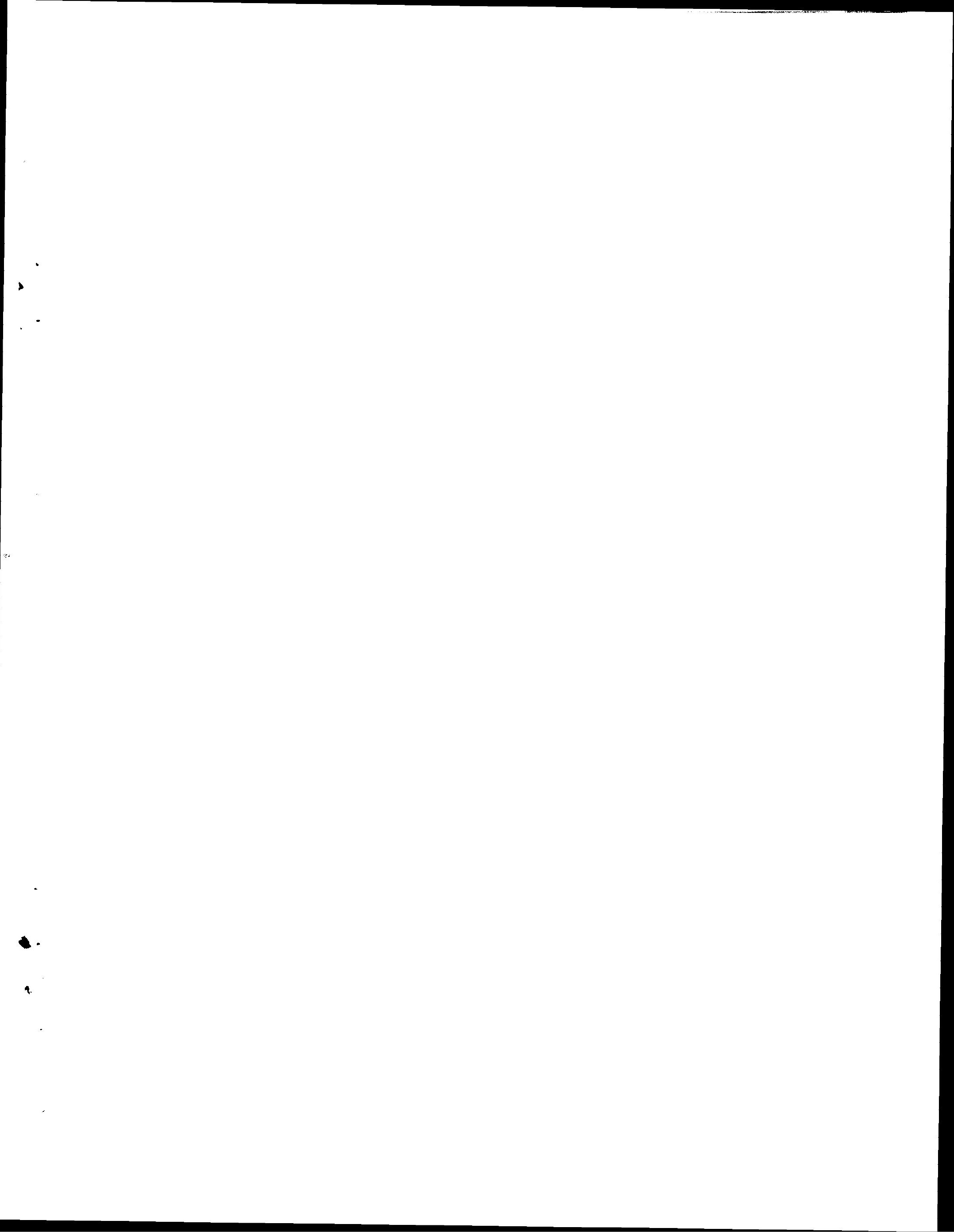


HANFORD TECHNICAL LIBRARY

NII

(1)

33679000564817

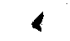

